\title{
Multiple light scattering in porous gallium phosphide
}

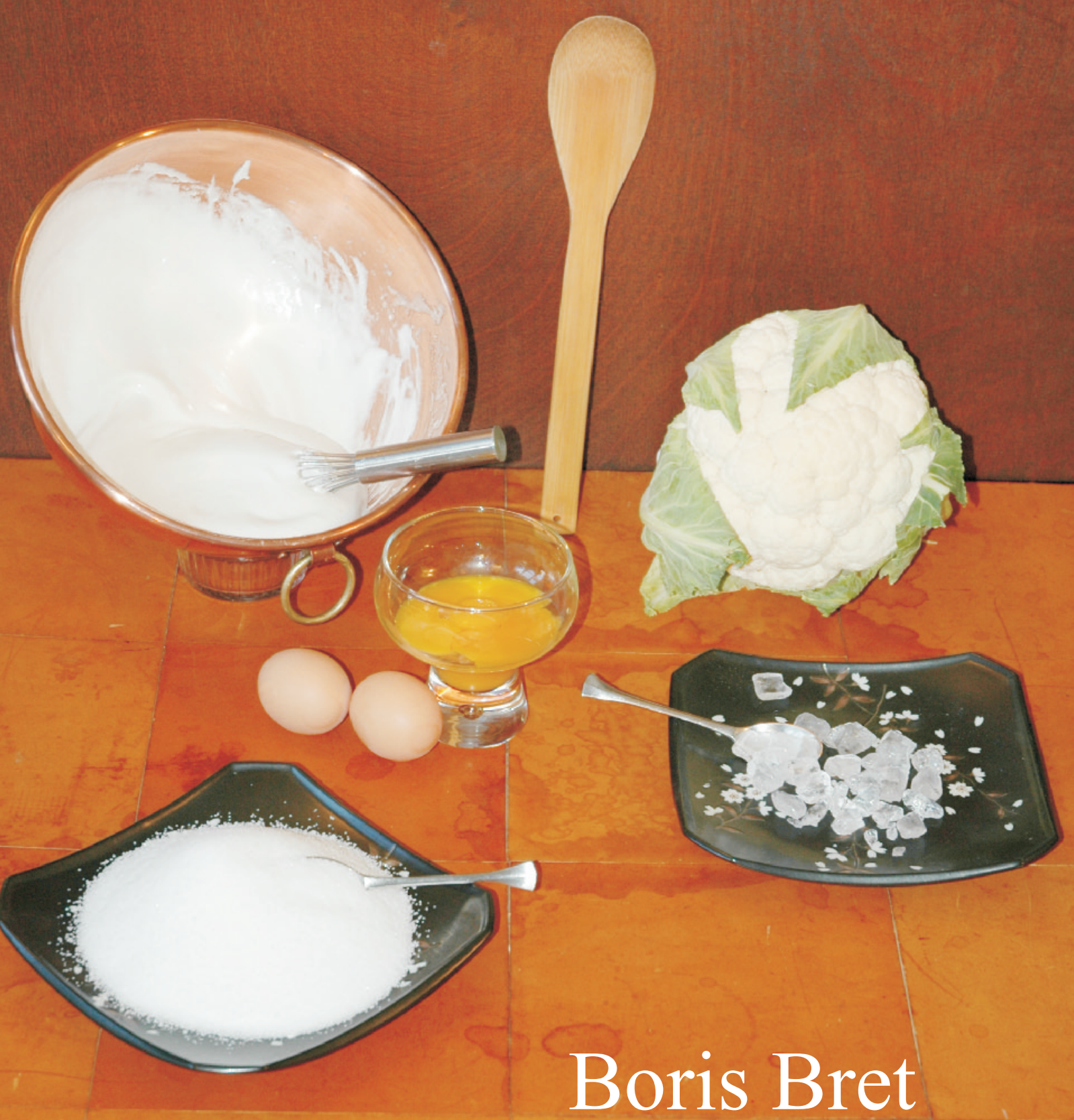


Promotiecommissie:

Promotor $\quad$ Prof. Dr. A. Lagendijk

Overige leden Prof. Dr. Ir. A. Bliek

Dr. T. W. Hijmans

Prof. Dr. J. J. Kelly

Prof. Dr. D. Lohse

Prof. Dr. W. L. Vos

Prof. Dr. W. J. van der Zande

The work described in this thesis is part of the research program of the

"Stichting Fundamenteel Onderzoek der Materie" (FOM),

which is financially supported by the

"Nederlandse Organisatie voor Wetenschappelijk Onderzoek" (NWO).

It was initiated in the group

Waves in Complex Media,

Van der Waals-Zeeman Instituut, Valckenierstraat 65,

1018 XE Amsterdam, The Netherlands,

and completed in the group

Complex Photonic Systems,

Faculty of Science and Technology,

and MESA+ Research Institute,

University of Twente, P.O. Box 217,

7500 AE Enschede, The Netherlands.

This thesis can be downloaded from

http://www.wavesincomplexmedia.com.

Cover: Multiple light scattering in the kitchen, by Boris Bret.

Printed by Print Partners Ipskamp, Enschede, The Netherlands.

ISBN: 90-365-2196-3 


\title{
MULTIPLE LIGHT SCATTERING IN POROUS GALLIUM PHOSPHIDE
}

\author{
PROEFSCHRIFT \\ ter verkrijging van \\ de graad van doctor aan de Universiteit Twente, \\ op gezag van de rector magnificus, \\ prof. dr. F.H.M. Zijm, \\ volgens besluit van het College voor Promoties \\ in het openbaar te verdedigen \\ op donderdag 14 juli 2005 om 13.15 uur
}

\section{door \\ Boris Paul Jean Bret}

geboren op 27 December 1978

te Parijs (Frankrijk). 
Dit proefschrift is goedgekeurd door:

Prof. Dr. A. Lagendijk 
À mes parents, Ariane et Jean-Paul, 



\section{Contents}

1 Introduction 9

$1.1 \quad$ Elastic interaction of light and matter . . . . . . . . . . . . . . 9

1.2 Interference in multiple scattering of light $\ldots \ldots \ldots \ldots \ldots$

1.3 On order and disorder $\ldots \ldots \ldots \ldots \ldots \ldots$

1.4 Strongly-scattering samples $\ldots \ldots \ldots \ldots \ldots$

1.5 Outline of the thesis . . . . . . . . . . . . . . . . . . . . . . 19

\begin{tabular}{|lll}
2 & Principles of multiple scattering theory & 21
\end{tabular}

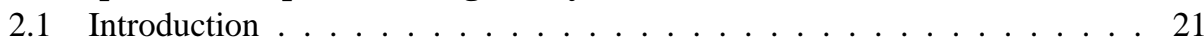

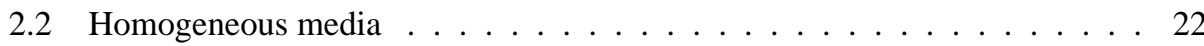

2.3 Single scattering. . . . . . . . . . . . . . . . . . . 23

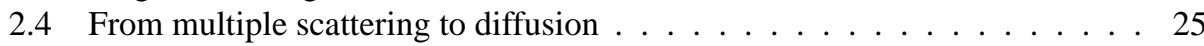

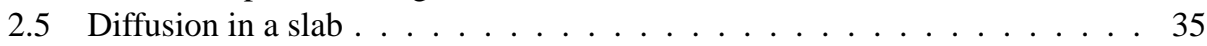

2.6 Enhanced backscattering $\ldots \ldots \ldots \ldots \ldots \ldots$

2.7 Conclusions . . . . . . . . . . . . . . . . . . . . . . 43

3 Chemistry of porous gallium phosphide 45

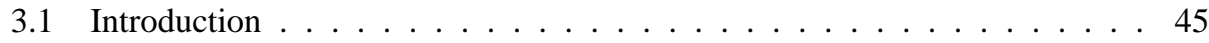

3.2 Electrochemical etching of $\mathrm{GaP} \quad \ldots \ldots \ldots \ldots$

3.3 Further chemical processing of porous $\mathrm{GaP} \ldots \ldots \ldots \ldots 2$

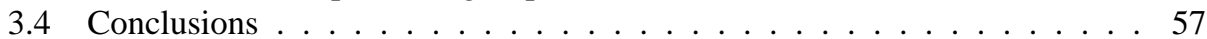

4 Diffusion at the interface $\quad 59$

4.1 Introduction . . . . . . . . . . . . . . . . . . . . 59

4.2 Theory of the interface of a diffusive medium . . . . . . . . . 59

4.3 Measuring the index of refraction of porous media . . . . . . . . . . . 64

4.4 Index of refraction versus porosity $\ldots \ldots \ldots \ldots \ldots$

4.5 Conclusions $\ldots \ldots \ldots \ldots \ldots \ldots \ldots \ldots \ldots \ldots \ldots$

\begin{tabular}{|lll}
5 & Strong scattering in porous GaP & 73
\end{tabular}

5.1 Introduction $\ldots \ldots \ldots \ldots \ldots \ldots \ldots \ldots \ldots \ldots \ldots$

5.2 Anodically etched samples $\ldots \ldots \ldots \ldots \ldots$. . . . . . . . . . . . 74

5.3 Increasing the pore size with chemical etching $\ldots \ldots \ldots \ldots \ldots$

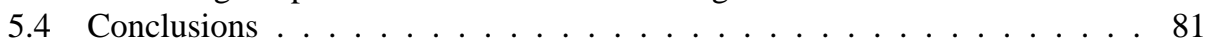


$6 \quad$ Anisotropic wave diffusion in porous GaP 83

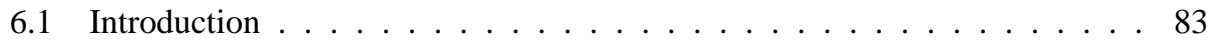

6.2 Generalizing diffusion to an anisotropic medium . . . . . . . . . . . . 85

6.3 Stationary anisotropic diffusion . . . . . . . . . . . . . . . . . 92

6.4 Dynamic anisotropic diffusion . . . . . . . . . . . . . . . . . . . 97

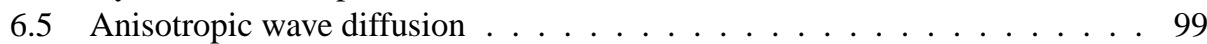

6.6 Conclusions . . . . . . . . . . . . . . . . . . . . 102

7 Capturing a light pulse in a short high-finesse cavity 103

7.1 Introduction . . . . . . . . . . . . . . . . 103

7.2 Introduction to the theory of a cavity . . . . . . . . . . . . . 107

7.3 Theoretical description of the pulse capture . . . . . . . . . . . . . 110

7.4 The variable input coupler . . . . . . . . . . . . . . . . . . 117

7.5 Our experimental methods . . . . . . . . . . . . . . . . . 119

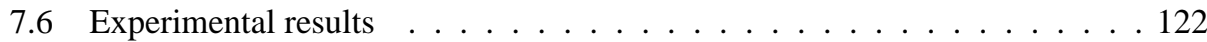

7.7 Conclusions . . . . . . . . . . . . . . . . . . 126

\begin{tabular}{ll}
\hline References & 127
\end{tabular}

\begin{tabular}{ll}
\hline Summary & 137
\end{tabular}

Samenvatting 139

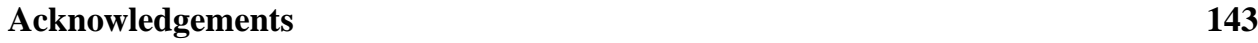




\section{Chapter 1}

\section{Introduction}

Sight is certainly one of the main senses of human beings. An enormous amount of information from our surroundings is obtained by our eyes, which are able to detect the color and the intensity of visible light, with a high angular resolution. Although this amount of information is contained in the light we can perceive, the mechanisms which lead to the color of an object are not apparent. What differentiates the blue from the sea of a lagoon, from a clear summer sky, or from your favorite land's ${ }^{1}$ flag? The first one is due to reflection, the second to single scattering, and the third to multiple scattering (in addition to an absorption/emission mechanism).

\subsection{Elastic interaction of light and matter}

\subsubsection{Single scattering}

Through homogeneous media, such as vacuum between the sun and the earth, air around us, or the glass of windows, light propagates in a straight line, or as a ray, with a single speed. This straight propagation is disturbed by inhomogeneities, or a change in the speed of light. The speed of light inside a material is usually specified through the refractive index $n$ of this material. The refractive index is the ratio of the speed of light in vacuum and inside the material. An interface between air and water for example gives rise to refraction, where the direction of light changes when propagating between two media of different refractive indices. An interface between air and a metal typically gives rise to a strong reflection of the light, of which we make everyday use with mirrors. Apart from a clear and planar interface between two otherwise homogeneous materials, examples of inhomogeneities are single atoms, molecules, droplets of water, glass spheres, or sugar powder.

There are two equivalent descriptions of the inhomogeneity of a medium. The refractive index can be described as a continuous variable, which varies with position. A physical example matching this description is the air above a fire, whose temperature fluctuations lead to density fluctuations of the air and to refractive index fluctuations. The propagation of light through the air above a fire is disturbed and makes an image seen through this air

\footnotetext{
${ }^{1}$ The French and Dutch flags are good examples, both containing the blue color.
} 

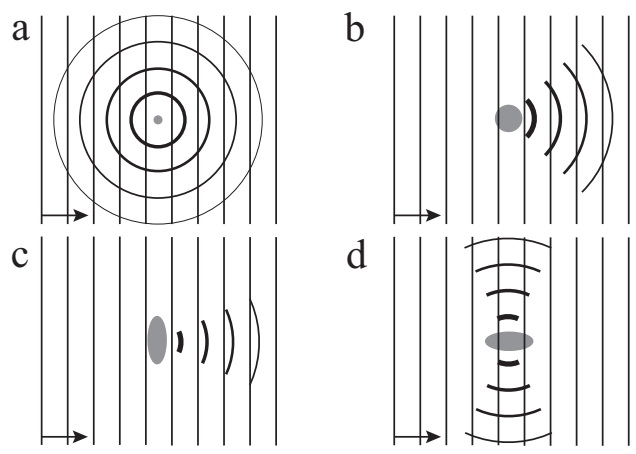

Figure 1.1: Cartoon of the scattering function of several scatterers depicted in grey, under illumination of a plane wave incident in the direction of the arrow. In (a), a Rayleigh scatterer, almost isotropi ${ }^{2}$. In (b), a Mie sphere, scattering preferentially in the forward direction. In (c) and (d), a non-spherical scatterer, whose scattering function depends on the orientation of the scatterer.

shudder. The refractive index can also be described as constant and homogeneous, within one material. The inhomogeneity then comes from inclusions of one material into another material of different refractive index. Mist is a region of air filled with very small water droplets, through which the vision is blurred. In this thesis, the description is used of a homogeneous material filled with inclusions of another material, which we call scatterers. The interaction of light with a scatterer is a scattering event, and it is in this thesis considered to be elastic: the wavelength of the light is not changed by scattering.

The regime in which light interacts only once with a scatterer is called single scattering. The main property of a scatterer can be specified as its scattering cross section $\sigma_{\text {sc }}$, which is the total intensity of light scattered by this scatterer, normalized to a surface. Three different categories of single scattering are usually described, depending on the size of the scatterer: Rayleigh scattering, Mie scattering, and geometrical optics. Rayleigh scattering [1-4] describes the interaction of light with scatterers of size much smaller than the wavelength of light $\lambda$, such as molecules or very fine powders. The scattering function, or the angular distribution of light after scattering, is isotropic in the long wavelength limit, as schematized in Fig. 1.1 a: a small scatterer is an isotropic scattere ${ }^{2}$. Rayleigh scattering is characterized by the dependence of the scattering cross section on the inverse of the fourth power of the wavelength of light: $\sigma_{\mathrm{sc}} \propto 1 / \lambda^{4}$. In the Rayleigh regime, the scattering of light is much more efficiently in the blue part of the visible spectrum than in the red part. The atmosphere contains very small scatterers, like molecules or aerosols, which give the blue color to the light scattered from the sky. The red sun at dawn and sunset is the transmitted light through a thick layer of atmosphere, where the blue part of the spectrum has been more efficiently scattered out than the red part. The limit of an infinitely-small scatterer compared to the wavelength of light is called the point scatterer and is the choice model for theories beyond single scattering, an example of which is given in chapter 2

\footnotetext{
${ }^{2}$ The scattering function of a Rayleigh scatterer has in fact a small angular dependence, depending on the polarization of the incident beam. Although the scattering function is not completely isotropic, it is still symmetric between forward and backward scattering.
} 
The geometrical-optics limit is used for scatterers of size much larger than the wavelength of light, where the well-known reflection and refraction (Snell's law) phenomena are sufficient to describe the propagation of light. In this small-wavelength limit, the scattering cross section of a scatterer equals twice its physical cross section. In geometrical optics, a ray stays a ray after scattering (i.e., after reflection or refraction).

In the intermediate category, when the size of the scatterer is close to the wavelength of light, only the spherical or cylindrical scatterer can be exactly solved. Mie scattering [3-5] describes the interaction of light with a sphere of arbitrary size, i.e., a Mie sphere. In the long and short wavelength limit, Mie scattering catches up with the Rayleigh scattering and geometrical optics respectively. A sphere of size close to the wavelength of light exhibits resonances at discrete wavelengths where the scattering is very efficient. At these resonances 3 , the scattering cross section of the Mie sphere greatly exceeds its physical cross section. The scattering function of a Mie sphere depends very much on the wavelength of light, whether or not a resonance of the sphere is excited. Mie spheres typically scatters more in the forward direction, as depicted in Fig. 1.1p, and are therefore anisotropic scatterers.

In the case of a non-spherical (and non-cylindrical) scatterer of size close to the wavelength of light, either an analytical solution for a collection of point scatterers modeling the real structure or a numerical solution for the exact scatterer is necessary. The intensity scattered from a non-spherical scatterer depends on angle (like the Mie sphere in Fig. 1.1p) but also on its orientation, unlike the Mie sphere (Figs. 1.1p and $1.1 \mathrm{~d}$ depict the same non-spherical scatterer, in two different orientations). A non-spherical scatterer also has resonances, depending on its shape and size [6].

The characteristic quantity of a single scatterer is its scattering cross section $\sigma_{\text {sc }}$. The characteristic quantity of a collection of scatterers of density $\rho$ is called the scattering mean free path $\ell_{\mathrm{sc}}$, and is the average distance between two consecutive scattering events. The intensity of the incident beam decays exponentially with the penetration depth inside the scattering material, with a typical decay length $\ell_{\mathrm{sc}}$. In the case of independent scattering, the scattering mean free path, to first order, is equal to

$$
\ell_{\mathrm{sc}}=\frac{1}{\rho \sigma_{\mathrm{sc}}} .
$$

The single-scattering regime holds when the size $L$ of the region of space where scatterers are present is smaller than the scattering mean free path $\ell_{\mathrm{sc}}$.

Note that if another effect, such as absorption, influences the propagation of light inside the material, the intensity of the incident beam decays according to the extinction length $\ell_{\mathrm{ex}}$. The extinction length is related to the absorption length $\ell_{\mathrm{a}}$ as $\ell_{\mathrm{ex}}^{-1}=\ell_{\mathrm{a}}^{-1}+\ell_{\mathrm{sc}}^{-1}$.

\subsubsection{Multiple scattering and diffusion}

In the limit where $\ell_{\mathrm{sc}}<L$, the single-scattering approximation breaks down, and multiple scattering occurs. At each scattering, the direction of light changes, according to the

\footnotetext{
${ }^{3}$ The so-called whispering-gallery modes are modes which can be described as rays at grazing incidence inside a large sphere, totally internally reflected by its surface. Such modes have very low intrinsic losses and are effectively very good cavity modes, where the light travels around inside the sphere for a long time.
} 
scattering function. Setting the wave nature of light aside, the propagation of light through a multiple-scattering medium can be described as a random walk. The average step size of this random walk is $\ell_{\mathrm{sc}}$ and the possible directions in which each following step can be performed are set by the scattering function of the scatterer. Depending on the scattering function, the direction of light is fully randomized after one or more $\ell_{\mathrm{sc}}$. A Mie sphere for example mainly scatters in the forward direction, and many scattering events are necessary to randomize the direction of light. Instead of a random walk with step size $\ell_{\mathrm{sc}}$ and anisotropic scattering function, an isotropic random walk can be described. The transport mean free path $\ell$ is defined as the average distance after which the intensity distribution is isotropic. The transport mean free path is the characteristic length in the regime of multiple scattering. In the absence of interference, $\ell$ becomes equal to the Boltzmann mean free path $\ell_{\mathrm{B}}$,

$$
\ell_{\mathrm{B}}=\frac{\ell_{\mathrm{sc}}}{1-\langle\cos \theta\rangle},
$$

where $\langle\cos \theta\rangle$ is the average cosine of the scattered angle, weighted by the scattering function. In the Rayleigh regime, the incident light is scattered symmetrically in the forward and in the backward direction, therefore $\langle\cos \theta\rangle=0$, and $\ell_{\mathrm{B}}=\ell_{\mathrm{sc}}$. Otherwise, the scatterers, such as a Mie sphere, scatter more in the forward than in the backward direction, so that $\langle\cos \theta\rangle>0$ and $\ell_{\mathrm{B}}>\ell_{\mathrm{sc}}$. In the regime ${ }^{4}$ where $\ell_{\mathrm{sc}} \leq \ell \ll L$, light is said to be diffuse, the energy density of light $W$ follows a diffusion equation

$$
\frac{\partial W}{\partial t}-D \nabla^{2} W=S
$$

where $D$ is the diffusion constant, characteristic of the speed at which light diffusely spreads out. The first part of chapter 2 gives a theoretical ground to the diffusion equation.

The diffusion equation is a very general and practical description of numerous complex systems in physics. The particles of a gas, the heat in solids [7], the neutrons in a nuclear reactor $[8,9]$, the coins in the euro zone all follow a diffusion equation. The diffusion of light has the following characteristic property: because light entering a diffusive material is scattered numerous times, the light emerging from the material is an average of the color of all the incident light, whatever the incident direction. Since ambient light usually contains all colors of the visible spectrum, e.g., from the sun or an incandescent lamp, the diffusive material appears white. All white materials owe their color to multiple light scattering. Famous examples of diffusive materials include clouds, white paint, ivory, snow, the fur of polar bears and paper. A kitchen typically gives the opportunity of observing, and even making, diffusive materials: milk, flour, cauliflower (see cover), beer foam or salt. Refined sugar comes in two forms, either sugar powder or candy sugar (see cover, respectively bottom left and right). Both forms of sugar are crystalline and identical except for the size of the crystals. Candy sugar is a large and transparent crystal. Sugar powder is a collection of small, but still transparent, crystals, whose surfaces each scatter light a little. Grinding a candy sugar into a powder turns it from transparent to a diffusive white. Similarly, the white of an egg is, mainly, transparent. After vigorous whipping, small air bubbles are introduced within a backbone made of the proteins of the white of the egg [10]. The whipped white becomes a white diffusive material (see cover, center left).

\footnotetext{
${ }^{4}$ The interference effects are here still neglected, but are detailed further on, in section 1.2
} 

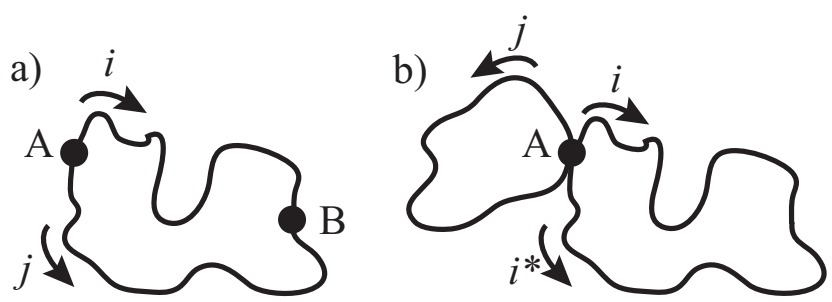

Figure 1.2: Possible paths for the light in a random material. In (a), two paths $i$ and $j$ lead from $A$ to $B$. In (b), two distinct paths $i$ and $j$ lead from $A$ back to $A, i^{*}$ is the time reverse of path $i$.

In the presence of a small but non-negligible absorption, for $\ell \ll \ell_{\mathrm{a}} \ll L$, the diffusion equation can be generalized, by adding a negative term next to the source in Eq. 1.3 . as $\left(-W / \tau_{\mathrm{a}}\right)$. The absorption time $\tau_{\mathrm{a}}$ takes into account the diffusion speed as $\tau_{\mathrm{a}} \equiv L_{\mathrm{a}}^{2} / D$. The absorption length $\ell_{\mathrm{a}}$ is the path length after which light is absorbed. The diffuse absorption length $L_{\mathrm{a}}$ is the average distance light propagates diffusively before being absorbed [11]:

$$
L_{a}=\sqrt{\frac{\ell \ell_{\mathrm{a}}}{3}} .
$$

Inside a diffusive and absorbing material, the diffuse absorption length is the penetration depth of the diffuse light.

\subsection{Interference in multiple scattering of light}

Although the diffusion equation is very general and describes the intensity of light in usual situations, the wave nature of light makes its propagation much more interesting than just diffusion.

In order to look at interference effects, the electromagnetic field has to be propagated, instead of the intensity. The intensity at a certain point is the norm squared of the field at this point. In Fig. 1.2 , two possible paths for light to travel between two distinct points A and $\mathrm{B}$ in a multiple-scattering medium are plotted. The intensity of light $I_{\mathrm{A} \rightarrow \mathrm{B}}$ at the point $\mathrm{B}$, due to light coming from the point $\mathrm{A}$ is the norm squared of the sum of the fields of all possible paths from $\mathrm{A}$ to $\mathrm{B}$ :

$$
I_{\mathrm{A} \rightarrow \mathrm{B}} \equiv\left(\sum_{i} E_{i}\right)\left(\sum_{j} E_{j}^{*}\right)=\sum_{i} E_{i} E_{i}^{*}+\sum_{i} \sum_{j \neq i} E_{i} E_{j}^{*}=\sum_{i} I_{i}+\text { interference, }
$$

where $E_{i}$ and $I_{i}$ are respectively the field and intensity of light propagating only along the path $i$. The sum of intensities along all the paths leads to the diffusion result, as in Eq. 1.3. The interference term can not in practice be calculated for a single configuration of the distribution of scatterers, but statistical properties (intensity distributions or spatial correlations for example) can be derived [12-15]. 


\subsubsection{Speckle}

In statistical physics, the self-averaging property of most random variables is primordial. Let us consider a random variable $\alpha$ with a probability distribution of finite variance (or mean-square). The central limit theorem states that the average over $N$ realizations of this random variable approaches asymptotically the average $\bar{\alpha}$ of the distribution function as

$$
\frac{1}{N} \sum_{i}^{N} \alpha_{i}=\bar{\alpha}+O\left(\frac{1}{\sqrt{N}}\right)
$$

where $O(x)$ is of the same order as $x$. If the distribution of the averages over $N$ realizations of the random variable tends to a Dirac delta function for $N \rightarrow \infty$, the random variable is called self-averaging. In practice ${ }^{5}$, this self-averaging means that the average over $N$ realizations of a random variable tends to the average of its distribution $\bar{\alpha}$.

The intensity $I_{i}$ along a path $i$ can be described as a random variable, if the scatterers are independent along each path. The intensity being only positive, its average does not vanish. The sum of intensities $\sum I_{i}$ in Eq. 1.5 therefore approaches its average value $\bar{I}_{i}$ times $N$. The intensity without interferences is self-averaging. The field $E_{i}$, on the other hand, is still a random variable, but in the complex plane: both amplitude and phase are random. The probability distribution of the amplitude follows the distribution for the intensity, but the probability distribution of the phase is constant: there is no preferential phase for the field from all the different paths through independent scatterers. The average value of the field $\bar{E}$ is therefore 0 and the fluctuations $O(1 / \sqrt{N})$ in Eq. 1.6 dominate the average over $N$ fields $E_{i}$ : the field is not self-averaging. The intensity $I_{A \rightarrow B}$, square of the average over $N$ fields, does not approach its average value $\bar{I}_{i}$, even for an infinite number $N$ of paths. The sum over $N$ realizations of a random variable of average 0 is of order $N / \sqrt{N}$. The double sum in Eq. 1.5 is therefore of order $(N / \sqrt{N})^{2}=N$, i.e., the same order as the sum of intensities $\sum I_{i}$. The interference term in Eq. 1.5 is therefore always of the same order as the diffuse intensity term, and either positive or negative. The intensity $I_{A \rightarrow B}$ therefore fluctuates with each realization of the $N$ paths. This fluctuation has a contrast of $100 \%$, i.e., both constructive interference of high intensity and destructive interference with intensity 0 are realized. This large fluctuation of the intensity of multiply-scattered waves with the disorder realization, or the position of point $\mathrm{B}$, is called speckle. A pattern of bright and dark spots is typical of the light from a coherent source, scattered by a disordered sample, as can be seen from Fig. 1.3 a.

By averaging over disorder ${ }^{6}$, i.e., averaging over multiple realizations of the scatterers distribution, the speckle pattern disappears, and only the diffuse intensity is recovered. The ensemble-averaged intensity is a self-averaging quantity whereas the intensity itself is not.

\subsubsection{Enhanced backscattering}

The ensemble-averaged intensity from a medium with multiple scattering does not present the typical fluctuations of speckles, but interference effects still remain. Instead of looking

\footnotetext{
${ }^{5}$ The stricter condition for the self-averaging, in terms of the Dirac delta function, is in particular necessary in the case of slowly-decaying distributions, such as Lévy distributions, whose moments are not finite [16].

${ }^{6}$ Also called ensemble averaging.
} 


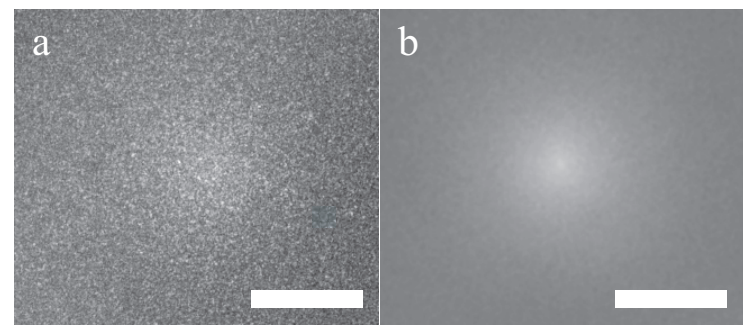

Figure 1.3: Angular-resolved backscattered intensity from a colloid of polystyrene spheres. In (a), no average over disorder realizations is done, only the speckle pattern is seen. In (b), the backscattered intensity is collected while the Brownian motion of the colloids average over the disorder realizations: the enhanced backscattering cone appears as a brighter spot in the center of the image. Scale bars $=5$ mrad. Courtesy of Wouter Peeters.

at the propagation from A to $\mathrm{B}$, as in Fig. $1.2 \mathrm{a}$, the propagation of a wave from A back to itself $I_{A \rightarrow A}$ is considered, as sketched in Fig. $1.2 \mathrm{p}$. In this case, each non-trivial 7 path has a distinct time-reversed path, such as $i$ and $i^{*}$ in Fig. 1.2p. The ensemble-averaged intensity is

$$
\begin{aligned}
\left\langle I_{\mathrm{A} \rightarrow \mathrm{A}}\right\rangle & \equiv\left\langle\left(\sum_{i} E_{i}\right)\left(\sum_{j} E_{j}^{*}\right)\right\rangle=\left\langle\sum_{i} E_{i} E_{i}^{*}\right\rangle+\left\langle\sum_{i} E_{i} E_{i^{*}}^{*}\right\rangle+\left\langle\sum_{i} \sum_{j \neq i, i^{*}} E_{i} E_{j}^{*}\right\rangle \\
& \simeq\left\langle\sum_{i} E_{i} E_{i}^{*}\right\rangle+\left\langle\sum_{i} E_{i} E_{i^{*}}^{*}\right\rangle=2\left\langle\sum_{i} I_{i}\right\rangle,
\end{aligned}
$$

where the ensemble-average of fields from path $j \neq i, i^{*}$ is 0 for independent scatterers. The fields from the time-reversed paths $i$ and $i^{*}$ are equal, provided the time-reversal symmetry is not broken [17]. Time-reversed paths always interfere constructively, and therefore double the intensity of light returning to the origin A, compared to the case when no interference is present.

If the point $\mathrm{A}$ is not inside the multiple-scattering medium, but is outside, in the far field, the factor 2 increase in intensity of $\left\langle I_{\mathrm{A} \rightarrow \mathrm{A}}\right\rangle$ compared to $\left\langle I_{\mathrm{A} \rightarrow \mathrm{B}}\right\rangle$ leads to the so-called coherent or enhanced backscattering (EBS). In the exact backscattered direction, where the incident and outgoing wave vectors are opposite, the interference of the time-reversed paths is fully constructive and the intensity is twice the diffusion expectation. In practice, the EBS is a cone of light, on top of the diffuse background, as showed in Fig. $1.3 \mathrm{p}$. At exact backscattering (in the center of Fig. 1.3p), a multiple-scattering sample reflects up to twice as much intensity as outside the EBS cone. The EBS cone is characteristic of the multiple scattering of waves, and its width is related to the two length scales involved, $\ell$ and $\lambda$ respectively, as $\lambda / \ell$. The EBS cone is fully derived and described in section 2.6 .

\subsubsection{Anderson localization}

The constructive interference of time-reversed paths leads to a more dramatic effect than the EBS. The higher intensity $I_{A \rightarrow A}$ compared to $I_{A \rightarrow B}$ means that the probability of a photon

\footnotetext{
${ }^{7} \mathrm{~A}$ trivial path is here meant as only containing one scattering event, before coming back to A.
} 
to return to its original position is larger than to diffuse away. In a material in which the interference effects are dominant, i.e., where the mean free path is close to the wavelength of light, the higher returning probability inhibits the propagation of light [18]. Light is said to be localized when the diffuse propagation disappears: $D=0$, or equivalently $\ell=0$. The transport mean free path $\ell$ is renormalized due to interference and therefore is not equal to the Boltzmann mean free path $\ell_{\mathrm{B}}$ in the case of waves. In a localizing medium, the energy density of light decreases exponentially with the distance $r$ to the source,

$$
W \propto \exp (-r / \xi),
$$

where $\xi$ is the localization length, or the typical length in which light is localized.

The concept of localization was first introduced by Anderson [19] in the case of random lattices (an amorphous metal for example) which inhibit the propagation of electrons. Later, localization was generalized to classical waves [20,21], which opened the path toward Anderson localization of light.

In less than 3 dimensions (3D), any amount of disorder localizes the wave, provided the limiting effects (like the finite thickness of the material, or absorption) are negligible $[22,23]$. In 3D, Anderson localization is a phase transition induced by disorder. The transition from the propagating to the localized $(\ell=0)$ state is expected to happen at the so-called Ioffe-Regel [24-26] criterion:

$$
k \ell_{\mathrm{B}} \simeq 1 \text {, or equivalently } k \ell_{\mathrm{sc}} \simeq 1 \text { for isotropic scatterers. }
$$

Apart from the difficult-to-achieve Ioffe-Regel criterion, Anderson localization of light remains elusive in $3 \mathrm{D}$ because of the always present, although usually negligible, optical absorption. Absorption introduces a cutoff in the distribution of path-length for light in a multiple-scattering medium. The diffuse absorption length $L_{\mathrm{a}}$ therefore breaks down localization [20,27] if $\xi>L_{\mathrm{a}}$. In addition, absorption of light in a multiple-scattering medium can easily be mistaken for Anderson localization. Indeed, the exponential decay of the energy density with distance from the source is as characteristic of localization as it is of absorption. Absorption and localization display similar effects on the EBS cone, as is detailed in section 2.6 and chapter 5. Dynamic diffusion [28] measurements and speckle statistics $[29,30]$ are expected to distinguish absorption from localization in a 3D medium.

\subsection{On order and disorder}

In this section, a non-exhaustive review of the wide range of interests in multiple scattering is presented, with an emphasis in optics. The field of multiple scattering presents two limiting cases. On the one hand, the scatterers can be arranged in an ordered, crystalline, structure. On the other hand, the distribution of scatterers can be random, corresponding to a completely amorphous, or disordered, material. Practical implementations of multiple scattering materials always lie somewhere in between these two extremes.

The analogy between electrons and photons, which initiated the search for Anderson localization of light [20,21] also led to the concept of photonic crystals [31,32]. Photonic crystals are the equivalent for photons of what the semiconductors are for electrons [33]: a material with a 3D periodic modulation of the refractive index, of period close to the wavelength of light. As it happens in the case of electrons in a semiconductor, a photonic crystal 
forbids the propagation of light in a range of wavelengths: a photonic band gap. The field of photonic crystals quickly expanded due to the expectations of numerous applications of photonic band gaps materials, inspired by semiconductor electronics [34]. Switching the properties of photonic crystals allows a very fast and active control over the propagation of light $[35,36]$. A qualitative difference between electrons and photons is the particle conservation. Photons can be absorbed and emitted, in opposition to electrons. Photonic crystals have been expected [31], and later shown [37,38], to inhibit, or more generally control, the spontaneous emission of embedded light sources. One of the most successful scheme to produce a large-scale 3D photonic crystal is the so-called 'inverse opal' $[39,40]$, where a crystallized colloid of polystyrene spheres (an opal) is inverted in order to obtain a crystal of air-spheres within a backbone of high refractive index.

The photonic crystals are in fact a very useful environment in which to have disorder. Close to the edge of a photonic band gap, the wave vector $k$ of light is reduced, and therefore makes the Ioffe-Regel criterion for Anderson localization, $k \ell_{\mathrm{B}} \simeq 1$, more easily attainable [32]. Adding a defect to an otherwise perfect photonic crystal opens an allowed state within the photonic band gap [41-43]. Such a 'nano-box' localizes light within the photonic band gap. The light escaping photonic crystals of macroscopic size has been shown to be diffuse but strongly directional $[44,45]$, indicating that even state-of-the-art photonic crystals have uncontrolled disorder $(\ell \sim 20 \mu \mathrm{m})$. Another example of medium combining both disorder and order characteristics is the quasi-crystal: a layered structure whose variable layer thickness follows for example a Fibonacci sequence. In such aperiodic but deterministic structure, both photonic stop gaps and 1D (quasi-)localization effects have been observed [46-48].

The purely disordered limit of multiple scattering is also flourishing. The diffusion approximation, and its generality, is known since the end of the $18^{\text {th }}$ century, but a renewed interest arose two centuries later over the diffusion regime with an underlying wave equation. Interference introduces fascinating effects in multiple light scattering [49-51], as section 1.2 already demonstrated.

Disorder in multiple scattering even raises hopes for some unexpected applications. The time-reversibility of acoustical waves allows to focus the waves through a multiplescattering medium and, e.g., destroy brain tumors [52]. A coherent wave is sent through a multiple-scattering medium, and the amplitude and phase of the scattered wave is recorde ${ }^{8}$ The recording of the complex acoustic field is played back, after time-reversal of the signal. The acoustic field then propagates in exactly the same path, but in time reverse, as the scattered field. Such recording and time-reversed play-back scheme is called an acoustic time-reversal mirror $[55,56]$. The time-reversal of the field scattered by a tumor in the brain, or a kidney stone, is focussed back on its origin. The amplification of the acoustic signal before play-back selectively heats up and destroys the alien scatterer.

Multiple-scattering materials have been proposed as a source for physically uncloneable functions [57]. A speckle pattern is characteristic of a particular scattering material and illumination, and can be coded in order to produce a personal and secret key, useable in cryptographic schemes.

Communication in free air in an empty world is easy. The propagation of electromag-

\footnotetext{
${ }^{8}$ Acoustic transducers automatically measure both amplitude and phase of acoustic waves. The same measure in optics requires a complicated interferometry setup $[53,54]$.
} 
netic waves between two antennas is straight. In a disordered world, where buildings, cars or mountains scatter all radio waves, the signal at the receiving antenna is blurred by speckle. An array of receiving antennas, which can resolve the speckle, allows to decode the original information, still contained in the speckle [58]. The application of the timereversal mirror to the communication in a disordered world was also shown to increase the selectivity and the rate of the information transmission [59].

The addition of a pumped gain medium inside a multiple-scattering material leads to the so-called random lasers $[60,61]$, where the feedback mechanism is offered by the multiple scattering. Random lasers of very small size [62,63] and low-threshold [64] have been reported.

Multiple scattering, which retain phase information, also preserves the subtle quantum information, as the quantum noise $[65,66]$.

Disorder has been shown to be an advantage in certain non-linear processes, where phase-matching is in principle primordial [67-70]. Perfect phase-matching means that all the light generated by a non-linear process interferes constructively, so that the efficiency of the process is very good. A non-perfect phase-matching limits the size of the domains in which interference of generated light is constructive. The use of a non-linear crystal of size bigger than these domains drastically decreases the efficiency of the non-linear process. In the case of a disordered material, the domains in which interference is constructive do not interfere destructively with each other, but randomly. The generated light from a non-linear disordered material is therefore like a speckle, originating from fields of random phase. In average, the intensity of such speckle is much higher than the intensity coming from just one domain 9 , and therefore allows the use of (cheaper) disordered non-linear materials to generate, with a good efficiency, harmonics. Strongly scattering porous gallium phosphide has been specifically used to enhance a second-harmonic-generation process $[67,68,71]$.

The holy Grail of the study of multiple-scattering in disordered media is the observation, characterization and further application of Anderson localization.

\subsection{Strongly-scattering samples}

In order to study diffusion, interference effects in multiple light scattering, or Anderson localization, suitable samples have to be made. Nature offers materials with a very wide range of scattering strength and properties. The most interesting interference effects in multiple scattering of light appear in materials with a very strong interaction between light and matter. Materials of ever stronger scattering strengths (i.e., where $k \ell$ is lower) are sought after. As previously illustrated in examples taken from the kitchen (in section 1.1.2), multiple scattering samples can typically be made in two ways: either grinding a transparent material into powder (such as the candy sugar into sugar powder) or incorporating bubbles of air into a transparent material (such as the white of the egg, whipped into a white stable foam).

Producing a strongly-scattering material typically follows one or the other of these production mechanisms. Powders of materials with high refractive index, such as titanium dioxide [72,73], zinc oxide [74,75], gallium arsenide [76], germanium [77-79] and sili-

\footnotetext{
${ }^{9}$ The intensity of this speckle is also smaller than in the case of a (quasi-)phase-matched non-linear crystal.
} 
con $[80,81]$, have all been shown to scatter light very strongly. The other mechanism, introducing air bubbles or holes into a material, has lately been used to produce the strongest scattering samples for visible light [81-84]. Gallium phosphide (GaP) is the semiconductor with the highest refractive index in the visible, namely $n=3.3$, and is transparent for red and yellow light. Etching in the right conditions [85-87] drills holes in GaP, or pores, of diameter comparable to the wavelength of light and in a random pattern. Chapter 3 describes the formation and optimization of the porous structure in GaP. Such a porous material scatters light very strongly, as is illustrated in chapter 5 .

In strongly-scattering media, where interference effects are expected to take a major role, the characterization of samples is of critical importance. Such a characterization is performed in chapters 4 and 5 Even for samples with very strong scattering (where $k \ell \simeq$ 3.5) the diffusion approximation, along with the EBS correction, holds surprisingly well, as chapter 5 shows.

Another very interesting property of the multiple-scattering materials we present in this thesis is the anisotropy of porous GaP. Anisotropy, as an angular-dependence of the mean free path or diffusion constant, can be seen both as a drawback and an advantage of strongly scattering samples. Of course, the diffusion approximation sketched in section 1.1 .2 is isotropic and can only fail to describe anisotropy. On the other hand, in a 3D material, the anisotropy decreases the dimension (a 1D or 2D material can be described as a 3D material with infinite anisotropy). Anderson localization of light is expected to arise more easily in a $3 \mathrm{D}$ anisotropic medium than in an isotropic one [88,89]. Both diffusion and wave diffusion properties of anisotropic porous $\mathrm{GaP}$ are presented and discussed in chapter 6 .

\subsection{Outline of the thesis}

This thesis presents a study on multiple light scattering in strongly-scattering porous GaP samples. The theory of diffusion and EBS on one hand, and the preparation of the strongly scattering samples on the other hand are necessary preliminaries to the experimental study. Effects such as the escape of the diffuse light through the interfaces, interference corrections of strongly-scattering samples, and macroscopic anisotropy in multiple light scattering are more particularly presented.

- Chapter 2 presents an introduction to the theory for multiple scattering of waves. The propagation of light in inhomogeneous media is described in terms of Green functions, scattering matrices and scattering diagrams. A Boltzmann equation for the transport of intensity in the multiple-scattering regime is derived, and leads to the diffusion equation. The diffusion equation is applied to the geometry of a slab in order to give predictions for actual experiments. Stationary diffusion is characterized by the transport mean free path $\ell$ whereas dynamic diffusion is characterized by the diffusion constant $D$. The EBS effect, the remaining of the interference in multiple scattering after ensemble averaging, is derived and commented on.

- In chapter 3, the processes for the formation of the strongly-scattering porous GaP samples are detailed. Electrochemical etching produces a layer of porous material, diffusive for light. The geometric properties of porous GaP can be varied depending on the etching conditions. Photochemical etching allows the removal of a bare 
GaP layer, remaining from electrochemical etching, to simplify the analysis of optical measurements. Further chemical etching increases the size of the pores, in a controlled way, thereby increasing the range of geometric and scattering properties achievable with porous $\mathrm{GaP}$.

- Chapter 4 focusses on the interface of a diffusive medium. The boundary conditions to the diffusion equation are derived and explained. The angular-dependence of the diffuse light escaping the material through the interface is shown to be characteristic of the effective refractive index of the material. We show that the refractive index of strongly-scattering porous GaP samples can be determined. The refractive index of porous $\mathrm{GaP}$ as a function of porosity does not follow the effective medium theories usually accepted.

- In chapter 5 the scattering properties of porous GaP are determined. The effects of the three etching steps from chapter 3 are quantified. Porous GaP samples are optimized, in the electrochemical-etching step and in the further chemical-etching step, for strong scattering. Optical absorption is shown to be negligible. Total transmission and EBS measurements are performed on samples with $k \ell \simeq 3.5$. No optical measurement on our samples presents a deviation from diffusion, expected at the onset of Anderson localization.

- In chapter 6 a macroscopic anisotropy in a multiple-scattering medium is studied. A hopping model is used to generalize the diffusion equation to a medium with an anisotropic diffusion constant. The expectations for stationary, dynamic diffusion, and EBS are derived in the anisotropic case, and are shown to all depend on the anisotropy in the diffusion constant. Anisotropic porous GaP samples are produced. The diffusion is shown to be anisotropic in these samples, from stationary and dynamic diffusion, and EBS measurements. The interpretation of stationary measurements in terms of a dynamic quantity, the (anisotropic) diffusion constant, is commented on.

The last chapter of this thesis does not treat of multiple light scattering or porous GaP. Nevertheless, this chapter has conceptual links to the scattering theory presented in chapter 2. In addition, the scheme of cavity-mode switching presented here can be easily generalized and adapted to the switching dynamics of an (Anderson) localized state.

- Chapter 7 addresses the subject of the capture of a light pulse in a short high-finesse cavity. In theory, a pulse can be totally coupled in a short cavity, provided the reflectivity of the input coupler is dynamically matched to the incident pulse shape. In the case of a high-finesse cavity, such pulse capture also compresses the frequency components of the incident pulse within one, thin, cavity mode. We realize experimentally this pulse capture scheme. The light inside the switched cavity is shown to have at the same time the highest intensity and the narrowest bandwidth compared to any stationary cavity.

Most of the results contained in this thesis can also be found in references [28,90-94]. 


\section{Chapter 2}

\section{Principles of multiple scattering theory}

In this chapter, I present a full derivation of multiple scattering theory needed to obtain experimental predictions. The reader who only wants a quick peak at the theoretical results is advised to jump directly to sections 2.5 and 2.6.2. The more theoretically-inclined reader is encouraged to follow the chapter from the beginning, which gives a fuller and more satisfying ground to the theoretical results.

\subsection{Introduction}

Light is an electromagnetical wave, yet in most everyday materials (milk, paint, or wood), it behaves in the same way as the variations of temperature in a room, or a drop of ink in a tank of water, i.e., according to a diffusion equation. This chapter derives how light goes from the free-space directional propagation to the diffusive transport in a medium with lots of scatterers. The first section introduces the concept of Green functions for describing the propagation of light, and first of all in a medium without scattering. The second section considers the interaction of light with one scattering potential. The T-matrix formalism for one scatterer is introduced and leads to the description of the simplest possible scatterer. The third section takes the step forward to multiple scattering of light. The assumption that interference of light plays no role leads to the Boltzmann equation. The, rather technical, derivation of this equation is inspired from Ref. [95] although a different, and more personal, approach is developed. The Boltzmann equation, through the radiative transfer equation, is approximated to obtain the well-known diffusion equation. Section four applies the diffusion equation to an experimental situation, to obtain the energy density profile in, the reflection of, and the transmission through, a diffusive slab. The last section relieves the assumption of the third section of the absence of interference, and derives the so-called enhanced backscattering effect (EBS). This last derivation is inspired from several sources [96-98] and corrects a mistake in the treatment of the internal reflection to preserve the enhancement factor of 2 in media with time-reversal conservation. 


\subsection{Homogeneous media}

Light being a wave, its propagation is described by a Helmholtz equation. The scalar Helmholtz equation in a homogeneous medium is

$$
\left(\nabla^{2}+k_{0}^{2}\right) \psi_{0}(\mathbf{r})=0
$$

where $\psi_{0}(\mathbf{r})$ is the amplitude of the scalar field, $\nabla^{2}$ the Laplacian operator, the amplitude of the wave vector $k_{0}=n_{0} \omega / c, \omega$ the angular frequency of light, $c$ its celerity in vacuum, $n_{0}$ the refractive index of the material, and $\mathbf{r}$ the position in space. The use of the scalar Helmholtz equation still captures the essential physics of multiple light scattering. The electric and magnetic fields are linearly related to the scalar field $\psi[95,99]$. The solution to the Helmholtz equation is the well-known plane wave with wave vector $\mathbf{k}_{0}$.

In order to describe anything else than free-space propagation, precious tools are the Green functions. The free-space Green function $g_{0}\left(\mathbf{r}, \mathbf{r}^{\prime}\right)$ is the amplitude of the field at position $\mathbf{r}$ coming from a source at position $\mathbf{r}^{\prime}$. The Green function $g_{0}\left(\mathbf{r}, \mathbf{r}^{\prime}\right)$ follows the equation

$$
\left(\nabla^{2}+k_{0}^{2}\right) g_{0}\left(\mathbf{r}^{\prime}, \mathbf{r}\right)=\delta\left(\mathbf{r}^{\prime}-\mathbf{r}\right)
$$

The medium being homogeneous, translation invariance implies that the Green function only depends on the difference between the two positions: $g_{0}\left(\mathbf{r}^{\prime}, \mathbf{r}\right)=g_{0}\left(\mathbf{r}^{\prime}-\mathbf{r}\right)$ and can also be written $g_{0}(\mathbf{r})$.

The solution to Eq.2.2 is easily found by applying Fourier transformation, and denoting p the Fourier space parameter:

$$
\left(-p^{2}+k_{0}^{2}\right) g_{0}(\mathbf{p})=1 .
$$

In Fourier space, the solution becomes

$$
g_{0}(\mathbf{p})=\frac{1}{k_{0}^{2}-p^{2}+i \epsilon},
$$

where an infinitesimally small imaginary part has been added. This addition solves the divergence at $p=k_{0}$, and allows complex analysis to be used in order to find the real space solution

$$
g_{0}(\mathbf{r})=-\frac{\exp \left(i k_{0} r\right)}{4 \pi r}
$$

which is recognized as a spherical wave, as was expected from a point source $\mathrm{Q}^{1}$

In order to obtain the field at a given position $\mathbf{r}$ from an ensemble of sources, the Green function has to be convoluted with the source field $\psi_{\mathrm{s}}(\mathbf{r})$, as

$$
\psi(\mathbf{r})=\int g_{0}\left(\mathbf{r}, \mathbf{r}^{\prime}\right) \psi_{\mathrm{s}}\left(\mathbf{r}^{\prime}\right) \mathrm{d} \mathbf{r}^{\prime} .
$$

\footnotetext{
${ }^{1}$ Note that the choice of the small positive imaginary part in Eq. 2.4 leads to take the spherical wave propagating outward, instead of inward, and therefore is the choice of causality [23,100].
} 


\subsection{Single scattering}

\subsubsection{Full Green function}

Scattering originates from an inhomogeneity in the refractive index of the medium. Considering a medium with refractive index $n_{0}$, the scattering potential of a region with refractive index $n(\mathbf{r})$ is $V\left(\mathbf{r}^{\prime}, \mathbf{r}\right) \equiv V(\mathbf{r}) \delta\left(\mathbf{r}^{\prime}-\mathbf{r}\right)=\left[n^{2}(\mathbf{r})-n_{0}^{2}\right](\omega / c)^{2} \delta\left(\mathbf{r}^{\prime}-\mathbf{r}\right)$. This position-dependent potential is found in the wave equation (see Eq. 2.1) as

$$
\left(\nabla^{2}+k_{0}^{2}\right) \psi(\mathbf{r})=\int V\left(\mathbf{r}, \mathbf{r}^{\prime}\right) \psi\left(\mathbf{r}^{\prime}\right) \mathrm{d} \mathbf{r}^{\prime}
$$

Eq. 2.7 is formally solved by using Eq. 2.6 and considering the term $V\left(\mathbf{r}, \mathbf{r}^{\prime}\right) \psi\left(\mathbf{r}^{\prime}\right)$ as the source:

$$
\psi(\mathbf{r})=\psi_{\text {in }}(\mathbf{r})+\int g_{0}\left(\mathbf{r}, \mathbf{r}_{1}\right) V\left(\mathbf{r}_{1}, \mathbf{r}_{2}\right) \psi\left(\mathbf{r}_{2}\right) \mathrm{d} \mathbf{r}_{1} \mathrm{~d} \mathbf{r}_{2},
$$

where $\psi_{\text {in }}(\mathbf{r})$, the incident wave, is a solution to Eq. 2.1 the homogeneous wave equation. Eq. 2.8 is known as the Lippman-Schwinger equation [101]. It is also possible to obtain a recursive equation for the full Green function, analogous to Eq. 2.8, known as the DysonSchwinger equation:

$$
g\left(\mathbf{r}^{\prime}, \mathbf{r}\right)=g_{0}\left(\mathbf{r}^{\prime}-\mathbf{r}\right)+\int g_{0}\left(\mathbf{r}^{\prime}-\mathbf{r}_{1}\right) V\left(\mathbf{r}_{1}, \mathbf{r}_{2}\right) g\left(\mathbf{r}_{2}, \mathbf{r}\right) \mathrm{d} \mathbf{r}_{1} \mathrm{~d} \mathbf{r}_{2} .
$$

\subsubsection{T-matrix}

By iterating Eq. 2.8, i.e., by developing the field in successive orders of the scattering potential $V$, a non-recursive equation is found:

$$
\psi(\mathbf{r})=\psi_{\text {in }}(\mathbf{r})+\int g_{0}\left(\mathbf{r}, \mathbf{r}_{1}\right) T\left(\mathbf{r}_{1}, \mathbf{r}_{2}\right) \psi_{\text {in }}\left(\mathbf{r}_{2}\right) \mathrm{d} \mathbf{r}_{1} \mathrm{~d} \mathbf{r}_{2},
$$

where the T-matrix $T\left(\mathbf{r}^{\prime}, \mathbf{r}\right)$ is defined as

$$
\begin{aligned}
T\left(\mathbf{r}^{\prime}, \mathbf{r}\right) \equiv & V\left(\mathbf{r}^{\prime}, \mathbf{r}\right)+\int V\left(\mathbf{r}^{\prime}, \mathbf{r}_{1}\right) g_{0}\left(\mathbf{r}_{1}, \mathbf{r}_{2}\right) V\left(\mathbf{r}_{2}, \mathbf{r}\right) \mathrm{d} \mathbf{r}_{1} \mathrm{~d} \mathbf{r}_{2} \\
& +\int V\left(\mathbf{r}^{\prime}, \mathbf{r}_{1}\right) g_{0}\left(\mathbf{r}_{1}, \mathbf{r}_{2}\right) V\left(\mathbf{r}_{2}, \mathbf{r}_{3}\right) g_{0}\left(\mathbf{r}_{3}, \mathbf{r}_{4}\right) V\left(\mathbf{r}_{4}, \mathbf{r}\right) \mathrm{d} \mathbf{r}_{1} \mathrm{~d} \mathbf{r}_{2} \mathrm{~d} \mathbf{r}_{3} \mathrm{~d} \mathbf{r}_{4}+\cdots
\end{aligned}
$$

The T-matrix is an expansion in orders of the scattering within the same scatterer. Taking only the first term in this expansion is known as the first-order Born approximation.

It is very useful to describe equations involving multiple scattering thanks to diagrams where integration is implicit. The notations for the diagrams are shown in Fig. 2.1. With this notation, Eq. 2.11 can be rewritten as

$$
\mathrm{x}=\mathrm{O}+\mathrm{0}-\mathrm{O}+\mathrm{O}-\mathrm{O}-\mathrm{O}+\cdots \text {. }
$$


o one scattering potential $V\left(\mathbf{r}^{\prime}, \mathbf{r}\right)$

$\mathrm{x}$ one T-matrix $T\left(\mathbf{r}^{\prime}, \mathbf{r}\right)$

free space Green function $g_{0}\left(\mathbf{r}^{\prime}, \mathbf{r}\right) \quad$ for scattering diagrams.

full Green function $g\left(\mathbf{r}^{\prime}, \mathbf{r}\right)$

...- link between identical particles $\mathbf{r}^{\prime}=\mathbf{r}$

\subsubsection{Point scatterers}

The T-matrix formalism allows to consider in one term the whole (internal) scattering possible from one particular scatterer. With this formalism, the point scatterer [102] is the easiest path to take. The point scatterer allows analytical solutions to be found for many multiple scattering problems, including the derivation of EBS in section 2.6. Other analytical solutions to the T-matrix include the point scatterer with gain [103], the Mie-sphere [3,104] and the plane scatterer [105].

Point scattering means scattering at exactly the position of the scatterer. The potential corresponding to the point scatterer is a Dirac delta function at the position $\mathbf{R}$ of the scatterer: $V\left(\mathbf{r}^{\prime}, \mathbf{r}, \omega\right) \equiv V(\omega) \delta\left(\mathbf{r}^{\prime}-\mathbf{R}\right) \delta(\mathbf{r}-\mathbf{R})$.

Introducing this point potential into the T-matrix definition, Eqs.2.11 or 2.12, gives the T-matrix for the point scatterer $t\left(\mathbf{r}^{\prime}, \mathbf{r}, \omega\right)$ :

$$
\begin{aligned}
t\left(\mathbf{r}^{\prime}, \mathbf{r}, \omega\right) \equiv & V(\omega) \delta\left(\mathbf{r}^{\prime}-\mathbf{R}\right) \delta(\mathbf{r}-\mathbf{R}) \\
& \times\left[1+g_{0}(\mathbf{R}, \mathbf{R}) V(\omega)+g_{0}(\mathbf{R}, \mathbf{R}) V(\omega) g_{0}(\mathbf{R}, \mathbf{R}) V(\omega)+\cdots\right] \\
= & \delta\left(\mathbf{r}^{\prime}-\mathbf{R}\right) \delta(\mathbf{r}-\mathbf{R}) \frac{V(\omega)}{1-V(\omega) g_{0}(\mathbf{R}, \mathbf{R})} .
\end{aligned}
$$

The point scatterer T-matrix in Fourier space is easily found as

$$
t\left(\mathbf{p}^{\prime}, \mathbf{p}, \omega\right)=\exp \left[i \mathbf{R} \cdot\left(\mathbf{p}-\mathbf{p}^{\prime}\right)\right] t(\omega) \quad \text { with } \quad t(\omega) \equiv \frac{V(\omega)}{1-V(\omega) g_{0}(\mathbf{R}, \mathbf{R})}
$$

In the case of point scatterers, the free-space Green function from one point back to itself has to be used. Strictly speaking, this $g_{0}(\mathbf{R}, \mathbf{R})$ is a divergence in the T-matrix, but is entirely due to the non-physical approach of an exact point-like scatterer. The divergence is solved by considering for example a scatterer of small but finite size, or by operating a cutoff in frequency space. The frequency-dependent term in the T-matrix of the point scatterer can be made explicit. For light in the vector case, the point scatterer has been shown to always display a resonance $[95,106]$. The regularization and frequency dependence of the T-matrix will neither be further discussed nor used in this thesis.

\subsubsection{Optical theorem}

A physical scatterer has to obey energy conservation. All energy which is removed from the incident wave should be either absorbed or scattered. In the following sections, only the case of elastic scattering, where no absorption takes places, will be developed. The extinction cross section $\sigma_{\mathrm{ex}}$ is the amount of light removed from the incident wave, normalized to 
a surface. The scattering cross section $\sigma_{\mathrm{sc}}$ is the amount of light, incident wave excluded, which flows through a sphere centered on the scatterer, in far-field, normalized to a surface.

In the absence of absorption, the scattering and extinction cross sections have to be equal. The energy conservation leads to the so-called optical theorem [107], which imposes a constrain on the T-matrix:

$$
\sigma_{\mathrm{ex}}=\frac{\operatorname{Im} T(\mathbf{k}, \mathbf{k}, \omega)}{k}=\int \frac{\left|T\left(\mathbf{k}^{\prime}, \mathbf{k}, \omega\right)\right|^{2}}{(4 \pi)^{2}} \mathrm{~d} \hat{\mathbf{k}}^{\prime}=\sigma_{\mathrm{sc}} .
$$

The extinction side of Eq. 2.15 shows how light is scattered from the wave vector $\mathbf{k}$ to the same k, therefore how much stays in the incident wave. The scattering side of Eq. 2.15 shows how much light scatters from the incident wave $\mathbf{k}$ into any other direction $\mathbf{k}^{\prime}$.

In the case of point scatterers, the optical theorem reduces to

$$
\frac{\operatorname{Im} t(\omega)}{\omega / c}=\frac{|t(\omega)|^{2}}{4 \pi} \text {. }
$$

\subsection{From multiple scattering to diffusion}

Having derived the behavior of one scatterer, through its T-matrix (Eq. 2.11, and the corresponding scattered field (Eq. 2.10), we can go on with multiple scattering, that is scattering by several distinct scatterers. The scatterers are considered equal, having the same T-matrix, and their density is $\rho$.

\subsubsection{Averaged full Green function}

We can now rewrite the Dyson-Schwinger equation (Eq. 2.9 in terms of scattering by a T-matrix, where all scattering events between different scatterers are taken into accoun 2

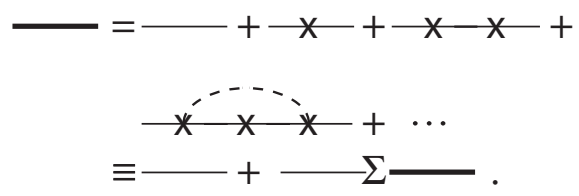

The so-called mass operator, or self-energy operato ${ }^{3}, \Sigma\left(\mathbf{r}^{\prime}, \mathbf{r}\right)$ is introduced to sum up all irreducible diagrams (those which can not be broken in two without breaking a dashed curve).

$$
\Sigma=x+x^{2} x+x+x^{x} x+\cdots
$$

The contributions to the self-energy operator, as seen from Eq. 2.18, include an infinite number of scattering events on a large number of different scatterers.

Knowing the exact position of all scatterers makes it in principle possible to calculate the field at all places. For a realistic number of scatterers $(\sim 1$ mole $)$ this exact solution

\footnotetext{
${ }^{2}$ Scattering twice consecutively on one single scatterer is forbidden, since this event is already accounted for in the T-matrix of the scatterer.

${ }^{3}$ Both names are originated from field theory.
} 
is nowhere near attainable by present day computers, nor practical. One particular distribution of scatterers gives rise to a speckle pattern: a field of average zero and of strongly fluctuating phase, amplitude and polarization. Speckle is better considered through its statistical properties [13-15,28, 108]. Speckle is not the subject of present derivation and is removed by taking the ensemble average of the full Green function. The operation $\langle\cdot\rangle$ is defined as the average over the position of all scatterers 4 . The medium being in average invariant by translation, the average quantities will not depend on the position, but on the distance between two points:

$$
\left\langle g\left(\mathbf{r}^{\prime}, \mathbf{r}\right)\right\rangle \equiv g\left(\mathbf{r}^{\prime}-\mathbf{r}\right) ; \quad\left\langle\Sigma\left(\mathbf{r}^{\prime}, \mathbf{r}\right)\right\rangle \equiv \Sigma\left(\mathbf{r}^{\prime}-\mathbf{r}\right) .
$$

One usual simplification is the so-called independent scattering approximation (ISA), which leads to neglect all but the lowest-order term in the series of $\Sigma\left(\mathbf{r}^{\prime}, \mathbf{r}\right)$. In Fourier space, the ISA is easily written $\Sigma(\mathbf{p})=\rho T(\mathbf{p}, \mathbf{p})$.

Applying the averaging on the recursive equation for the full Green function, Eq. 2.17 gives

$$
g\left(\mathbf{r}^{\prime}\right)=g_{0}(\mathbf{r})+\int g_{0}\left(\mathbf{r}-\mathbf{r}_{1}\right) \Sigma\left(\mathbf{r}_{1}-\mathbf{r}_{2}\right) g\left(\mathbf{r}_{2}\right) \mathrm{d} \mathbf{r}_{1} \mathrm{~d} \mathbf{r}_{2} .
$$

As was done to obtain the solution to the free-space Green function (Eq. 2.4), the average full Green function can be Fourier transformed, and symbolically solved 5 ;

$$
\begin{aligned}
& g(\mathbf{k})=g_{0}(\mathbf{k})+g_{0}(\mathbf{k}) \Sigma(\mathbf{k}) g(\mathbf{k}) \\
& g(\mathbf{k})=\left[g_{0}(\mathbf{k})^{-1}-\Sigma(\mathbf{k})\right]^{-1}=\left[k_{0}^{2}-p^{2}-\Sigma(\mathbf{k})\right]^{-1} .
\end{aligned}
$$

It is immediately clear from Eq. 2.22 that the solution to the average full Green function is analogous to the solution to the free-space Green function, in Eq.2.4 In the case of point scatterers, the wave vector can be explicitly renormalized as $\mathbf{K}$, with $K^{2} \equiv k_{0}^{2}-\Sigma$ :

$$
g(\mathbf{r})=-\frac{\exp (i K r)}{4 \pi r} .
$$

The real part of the renormalized wave vector leads to a different refractive index for the material. In fact, a material with an index of refraction $n$ different than 1 is a vacuum full of scatterers where the wave vector $\mathbf{K}$ induces $n_{0}$. The imaginary part of $\mathbf{K}$ leads to extinction, and is therefore related to the extinction mean free path $\ell_{\mathrm{ex}}: K \equiv n_{0} \omega / c+i / 2 \ell_{\mathrm{ex}}$.

The calculated average full Green function does not describe diffusion. It only describes how an incident wave penetrates in the medium, without tracking the light after it has been

\footnotetext{
${ }^{4}$ In practice, the ensemble averaging can be provided by several effects. In a colloidal suspension for example, the Brownian motion of the particles quickly and continuously randomizes the position of the scatterers. In a solid sample, like the porous $\mathrm{GaP}$ under study in this thesis, there is no Brownian motion. Ensemble averaging is then obtained by averaging over several measurements performed at different positions in the sample, or by rotating the sample. In the case of stationary measurements, a pulsed source can be advantageously used. The coherence length of the light pulse sets the distance between two adjacent regions which do not interfere one with the other. If the sample size is much larger than the coherence length, the number of these independent regions give the ensemble averaging.

${ }^{5}$ The infinitesimally small imaginary offset is dropped since $\Sigma$ is itself imaginary.
} 
scattered. The intensity of the non-scattered part of the incident beam through the medium is usually called the coherent transmission, and it follows the exponential decay

$$
\frac{I_{\text {coh }}}{I_{0}}=|\exp (i K z)|^{2}=|\exp [-\operatorname{Im}(K) z]|^{2} \equiv \exp \left(-z / \ell_{\mathrm{ex}}\right),
$$

where $z$ is the depth inside the medium, and $I_{\mathrm{coh}} / I_{0}$ is the intensity in the coherent beam, normalized to the incident intensity.

The term 'coherent transmission' or 'coherent beam', and its opposite 'incoherent beam' for the scattered wave, should not be taken literally. Coherence is the capacity of a wave to interfere. The (multiply) scattered wave keeps, as much as the remaining of the incident beam, its coherence, as can be shown from the effect of speckles or, as will be shown later, enhanced backscattering.

Measuring $\left\langle g\left(\mathbf{r}^{\prime}, \mathbf{r}\right)\right\rangle$ in a real experiment will require to average fields over disorder. A normal light sensitive detector only measures intensity. An interferometric setup allows both amplitude and phase information to be recorded. Averaging such interferometric data can lead to strictly observing the averaged full Green function. In practice, the 'coherent transmission' can more easily be measured by looking at the exact same wave vector in transmission than the incident wave [78].

\subsubsection{Intensity in the multiple scattering regime}

In the previous section, only the averaged full Green function has been calculated. The (multiply) scattered wave can not be described in this approach and the propagation of the intensity has to be explicitly considered. In order to obtain the multiply scattered intensity, the ensemble average of the norm squared of the field, $\left\langle\psi(\mathbf{r}) \psi^{*}(\mathbf{r})\right\rangle$, has to be considered.

The diagrams which now have to be drawn comprise two lines, the upper one being a Green function, and the lower one being the complex conjugate of a Green function. The intensity propagator, which is just a Green function for the intensity, is defined by

$$
G\left(\mathbf{r}_{1}, \mathbf{r}_{2} ; \mathbf{r}_{3}, \mathbf{r}_{4}\right) \equiv g\left(\mathbf{r}_{1}, \mathbf{r}_{2}\right) \times g\left(\mathbf{r}_{3}, \mathbf{r}_{4}\right) .
$$

The first few diagrammatic terms of the intensity propagator are

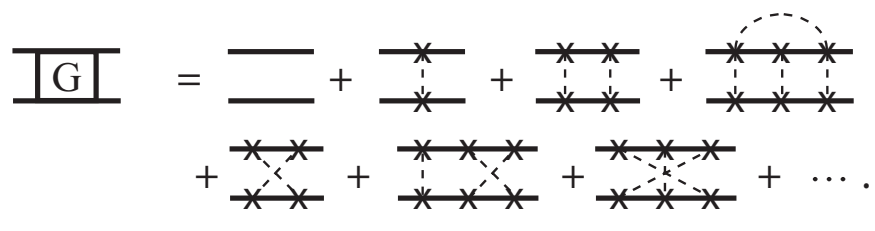

It is useful to describe also the intensity propagator $R$ similar to $G$ but without the incoming and outgoing Green functions $G \equiv\left(g \times g^{*}\right) R\left(g \times g^{*}\right)$. Using $R$ allows the calculation of the intensity of light generated by an incoming field according to

$$
\begin{aligned}
& \langle I(\mathbf{r})\rangle \equiv\left\langle\psi(\mathbf{r}) \psi^{*}(\mathbf{r})\right\rangle=\left\langle\psi_{\mathrm{inc}}(\mathbf{r})\right\rangle\left\langle\psi_{\mathrm{inc}}^{*}(\mathbf{r})\right\rangle \\
& \quad+\int\left\langle g\left(\mathbf{r}, \mathbf{r}_{\mathbf{1}}\right)\right\rangle\left\langle g^{*}\left(\mathbf{r}, \mathbf{r}_{\mathbf{3}}\right)\right\rangle\left\langle R\left(\mathbf{r}_{\mathbf{1}}, \mathbf{r}_{\mathbf{2}} ; \mathbf{r}_{\mathbf{3}}, \mathbf{r}_{\mathbf{4}}\right)\right\rangle\left\langle\psi_{\mathrm{inc}}\left(\mathbf{r}_{\mathbf{2}}\right)\right\rangle\left\langle\psi_{\mathrm{inc}}^{*}\left(\mathbf{r}_{\mathbf{4}}\right)\right\rangle \mathrm{d} \mathbf{r}_{\mathbf{1}} \mathrm{d} \mathbf{r}_{\mathbf{2}} \mathrm{d} \mathbf{r}_{\mathbf{3}} \mathrm{d} \mathbf{r}_{\mathbf{4}} .
\end{aligned}
$$

An equivalent relation has already been stated for the field in Eq. 2.10 
Similarly to the self-energy operator, containing all irreducible diagrams present in the full Green function (see Eq. 2.18, the sum of all irreducible diagrams in the intensity propagator is identified as $U\left(\mathbf{r}_{1}, \mathbf{r}_{2} ; \mathbf{r}_{3}, \mathbf{r}_{4}\right)$ :

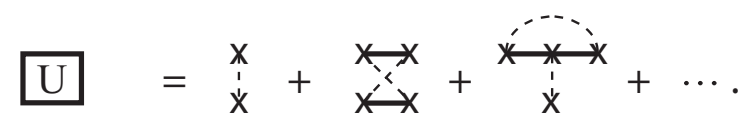

The relation between the intensity propagator $G$ and the sum of its irreducible terms $U$, written in symbolic form and ensemble averaged, also known as the Bethe-Salpeter equation, is

$$
\langle G\rangle=\langle g\rangle \times\left\langle g^{*}\right\rangle+\left(\langle g\rangle \times\left\langle g^{*}\right\rangle\right)\langle U\rangle\langle G\rangle .
$$

The solution to the intensity propagator still being impossible, successive approximations have to be made.

The first approximation neglects all but the lowest-order term in the series of $\langle U\rangle$. Since this lowest order term comprises only one scatterer, its contribution is linear in order of the density. This approximation is therefore known as the low-density approximation. The first term in $\langle U\rangle$ leads to the propagation of intensity from scatterer to scatterer, and is effectively considering no effect of interference in the intensity. The low-density approximation will therefore lead, in the following subsection, to diffusion. This first order term in $\langle U\rangle$ leads to consider a whole series of diagrams in $\langle R\rangle$, called the Ladder diagrams:

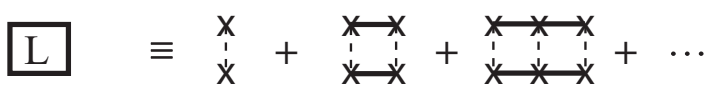

$$
\begin{aligned}
& \left.=\begin{array}{c}
x \\
1 \\
x
\end{array}+\begin{array}{c}
x \\
1 \\
1
\end{array}\right] \text {. }
\end{aligned}
$$

The second approximation keeps, along with the Ladder terms, a whole series of diagrams [109-111] in $\langle R\rangle$, called the most-crossed diagrams $\langle C\rangle$ :

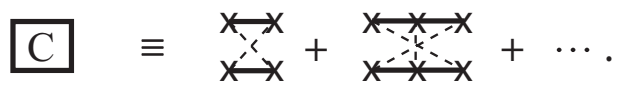

As was already intuitively understood, the first interference effect which remains after ensemble averaging corresponds to the time-reversed paths, which is what the most-crossed diagrams account for. This second approximation, $\langle R\rangle=\langle L\rangle+\langle C\rangle$, which gives rise to the enhanced backscattering, is developed in the next section.

Anderson localization of light requires a more subtle approximation and inclusion of the most-crossed diagrams, along with the first term of $\langle L\rangle$ in $\langle U\rangle$, and not in $\langle R\rangle$ [112-114].

\subsubsection{Boltzmann equation}

The first approximation is keeping only the Ladder terms $\langle L\rangle$ in the intensity propagator $\langle R\rangle$. 
Along with the definition of the Ladder terms in Eq. 2.30, a straightforward recursive equation is written. This recursive equation reads, in Fourier space, as

$$
\begin{aligned}
L\left(\mathbf{p}_{\mathbf{1}}, \mathbf{p}_{\mathbf{2}} ; \mathbf{p}_{\mathbf{3}}, \mathbf{p}_{\mathbf{4}}\right)= & T\left(\mathbf{p}_{\mathbf{1}}, \mathbf{p}_{\mathbf{2}}\right) T^{*}\left(\mathbf{p}_{\mathbf{3}}, \mathbf{p}_{\mathbf{4}}\right) \\
+\iiint \iint & T\left(\mathbf{p}_{\mathbf{1}}, \mathbf{p}_{\mathbf{a}}\right) T^{*}\left(\mathbf{p}_{\mathbf{3}}, \mathbf{p}_{\mathbf{c}}\right) g\left(\mathbf{p}_{\mathbf{a}}, \mathbf{p}_{\mathbf{b}}\right) g^{*}\left(\mathbf{p}_{\mathbf{c}}, \mathbf{p}_{\mathbf{d}}\right) \\
& \times L\left(\mathbf{p}_{\mathbf{b}}, \mathbf{p}_{2} ; \mathbf{p}_{\mathbf{d}}, \mathbf{p}_{\mathbf{4}}\right) \mathrm{d} \mathbf{p}_{\mathbf{a}} \mathrm{d} \mathbf{p}_{\mathbf{b}} \mathrm{d} \mathbf{p}_{\mathbf{c}} \mathrm{d} \mathbf{p}_{\mathbf{d}} .
\end{aligned}
$$

The averaged Ladder contribution $\langle L\rangle$ is invariant by translation. This invariance leads to the conservation of momentum, which can here be written as $\mathbf{p}_{\mathbf{1}}+\mathbf{p}_{\mathbf{3}}=\mathbf{p}_{\mathbf{2}}+\mathbf{p}_{\mathbf{4}}$. The translation invariance is used to simplify the equation of $\langle L\rangle$ according to

$$
\left\langle L\left(\mathbf{p}_{1}, \mathbf{p}_{2}, \mathbf{p}_{3}, \mathbf{p}_{4}\right)\right\rangle \equiv \delta\left(\mathbf{p}_{\mathbf{1}}-\mathbf{p}_{\mathbf{2}}+\mathbf{p}_{\mathbf{3}}-\mathbf{p}_{\mathbf{4}}\right) L\left(\mathbf{p}, \mathbf{p}^{\prime}, \mathbf{q}\right),
$$

where $\mathbf{p}, \mathbf{p}^{\prime}$ and $\mathbf{q}$ are the three independent Fourier vectors resulting, which are redefined, for later convenience, as:

$$
\left\{\begin{array}{l}
\mathbf{p}_{1}=\mathbf{p}+\mathbf{q} / 2 \\
\mathbf{p}_{2}=\mathbf{p}^{\prime}+\mathbf{q} / 2 \\
\mathbf{p}_{3}=-\mathbf{p}+\mathbf{q} / 2 \\
\mathbf{p}_{\mathbf{4}}=-\mathbf{p}^{\prime}+\mathbf{q} / 2
\end{array}\right.
$$

The integral of Eq.2.32 is still difficult to do because of the presence of the two Green functions. For the purpose of the integration, they are included in the function to calculate, according to

$$
M\left(\mathbf{p}, \mathbf{p}^{\prime}, \mathbf{q}\right) \equiv g(\mathbf{p}+\mathbf{q} / 2) g^{*}(\mathbf{p}-\mathbf{q} / 2) L\left(\mathbf{p}, \mathbf{p}^{\prime}, \mathbf{q}\right)
$$

The equation for $M$ reads, after integration over $\mathbf{p}_{\mathbf{c}}$ and with $\mathbf{p}_{\alpha} \equiv \mathbf{p}_{\mathbf{a}}-\mathbf{q} / 2$,

$$
\begin{aligned}
& M\left(\mathbf{p}, \mathbf{p}^{\prime}, \mathbf{q}\right)=g(\mathbf{p}+\mathbf{q} / 2) g^{*}(\mathbf{p}-\mathbf{q} / 2) \\
& \quad \times\left[\rho T T^{*}+\rho \int T\left(\mathbf{p}+\mathbf{q} / 2, \mathbf{p}_{\alpha}+\mathbf{q} / 2\right) T^{*}\left(\mathbf{p}-\mathbf{q} / 2, \mathbf{p}_{\alpha}-\mathbf{q} / 2\right) M\left(\mathbf{p}_{\alpha}, \mathbf{p}^{\prime}, \mathbf{q}\right) \mathrm{d} \mathbf{p}_{\alpha}\right],
\end{aligned}
$$

where the first term, product of the two T-matrixes, has been written symbolically $T T^{*}$, for readability, and because this term stays as a constant, $T T^{*} \equiv\left\langle T\left(\mathbf{p}+\mathbf{q} / 2, \mathbf{p}^{\prime}+\mathbf{q} / 2\right) T^{*}(\mathbf{p}-\right.$ $\left.\left.\mathbf{q} / 2, \mathbf{p}^{\prime}-\mathbf{q} / 2\right)\right\rangle$.

In order to pursue the integration, the product of the two Green functions has to be expanded, as

$$
g g^{*}=\frac{g-g^{*}}{1 / g^{*}-1 / g} .
$$

The product of the Green functions can equivalently be written as $g\left(\omega^{+}, \mathbf{p}^{+}\right) g\left(\omega^{-}, \mathbf{p}^{-}\right)$ [115] where $\omega^{ \pm} \equiv \omega \pm \Omega / 2 \pm i \epsilon$ and $\mathbf{p}^{ \pm} \equiv \mathbf{p} \pm \mathbf{q}$, in order to obtain dynamic quantities. Light is described as having two distinct time characteristics. The frequency of the light itself is $\omega$ and the frequency of its envelope, describing transport, is $\Omega$. Analogously, light has two distinct spatial characteristics, $\mathbf{p}$ its wave vector, and $\mathbf{q}$ the momentum of its envelope. 
The difference of the inverse of the two Green functions, in Eq. 2.37, can be explicitly written thanks to the solution for the full Green function found in Eq. 2.22.

$$
\begin{aligned}
\frac{1}{g\left(\omega^{-}, \mathbf{p}^{-}\right)}-\frac{1}{g\left(\omega^{+}, \mathbf{p}^{+}\right)}= & \left(\frac{\omega-\Omega / 2}{c}\right)^{2}-\left(\frac{\omega+\Omega / 2}{c}\right)^{2}-\left(\mathbf{p}-\frac{\mathbf{q}}{2}\right)^{2} \\
& +\left(\mathbf{p}+\frac{\mathbf{q}}{2}\right)^{2}-\Sigma\left(\omega^{-}, \mathbf{p}^{-}\right)+\Sigma\left(\omega^{+}, \mathbf{p}^{+}\right) \\
= & 2 \mathbf{p} \cdot \mathbf{q}-2 \frac{\Omega \omega}{c^{2}}+2 i \Delta \Sigma,
\end{aligned}
$$

with the definition $\Delta \Sigma \equiv\left[\Sigma\left(\omega^{+}, \mathbf{p}^{+}\right)-\Sigma\left(\omega^{-}, \mathbf{p}^{-}\right)\right] / 2 i$. In the same way, the corresponding shorthand $\Delta g$ is defined as $\Delta g \equiv\left[g\left(\omega^{+}, \mathbf{p}^{+}\right)-g\left(\omega^{-}, \mathbf{p}^{-}\right)\right] / 2 i$.

Eq. 2.36 becomes

$$
\begin{aligned}
{[-i \mathbf{p}} & \left.\cdot \mathbf{q}+i \frac{\Omega \omega}{c^{2}}+\Delta \Sigma\right] M\left(\mathbf{p}, \mathbf{p}^{\prime}, \mathbf{q}\right) \\
& =\Delta g\left[\rho T T^{*}+\rho \int T\left(\mathbf{p}^{+}, \mathbf{p}_{\alpha}^{+}\right) T^{*}\left(\mathbf{p}^{-}, \mathbf{p}_{\alpha}^{-}\right) M\left(\mathbf{p}_{\alpha}, \mathbf{p}^{\prime}, \mathbf{q}\right) \mathrm{d} \mathbf{p}_{\alpha}\right] .
\end{aligned}
$$

This last equation has to be interpreted. First of all, the function $M\left(\mathbf{p}, \mathbf{p}^{\prime}, \mathbf{q}\right)$ is an intensity propagator, including the Ladder diagrams and further propagation, in the direction $\mathbf{p}$ (See Eq. 2.35, similar to a specific intensity. The $i \Omega$ term in the left hand side of Eq. 2.39 can be interpreted as a time derivative in Fourier space, whereas the $i \mathbf{p} \cdot \mathbf{q}$ is a hydrodynamic flow term, containing a gradient. The $\Delta \Sigma$ is in fact the imaginary part of $\Sigma$, which was already seen in Eqs. 2.22 through 2.24 and attributed to extinction. The first term of the right hand side of Eq. 2.39 is independent of the intensity and therefore is a source term. The second term in the rhs reflects the scattering in the direction $\mathbf{p}$, from other directions.

It is important to note that in order to describe Eq. 2.39 as a time derivative plus a flow term, etc, a typical time has to be introduced. Phenomenologically, this typical time is the average travel time between two scattering events, depending on the mean free path and the phase velocity. It has been shown $[95,116,117]$ that this phenomenological assumption is wrong in the case of light since it forgets the dwell time in the scatterer. The rigorous time to be used is the mean free time $t_{\mathrm{mf}} \equiv \ell / v_{\mathrm{E}}$. The energy velocity $v_{\mathrm{E}}$ is described in more detail in the dynamic diffusion derivation (see section 2.4.4 and Eq. 2.54).

The physical interpretation of Eq. 2.39 can be summed up in

$$
\left[\frac{\partial}{\partial t}+\mathbf{v} \cdot \nabla+\text { scattering out }\right] I_{\mathbf{v}}(\mathbf{q}, t)=\text { source }+ \text { scattering in. }
$$

This equation is recognized as a Boltzmann equation, which rules the transport of classical particles. This Boltzmann equation is also known as a radiative transport equation, which is successfully used as the first multiple-scattering approximation [118].

The Boltzmann equation, although already a strong approximation to the full intensity propagation problem, can not be solved analytically. Numerous simulations [119] have been based on such a radiative transport equation, more particularly in astrophysics. The next step in order to obtain analytical solutions to a multiple-scattering problem resides in the diffusion approximation, which is now described. 


\subsubsection{Diffusion approximation}

The low-density approximation, which has already been used to retain only the ladder diagrams in the intensity propagator, has the following implication: the $\Sigma$ term in $\Delta g$ has to be neglected, that is, taking the free-space Green function: $\Delta g \rightarrow \Delta g_{0}$. In addition, the size of the scatterers is assumed to be much smaller than the macroscopic length scales describing the transport of light, which are represented by $\mathbf{q}$ in Fourier space. It is therefore possible to consistently neglect $\mathbf{q}$ in front of $\mathbf{p}$ in $\Delta \Sigma, \Delta g$ and the T-matrix product $T T^{*}$. The interpretation of this last approximation is that the scatterers see each others in far field.

Eq. 2.39 becomes

$$
\begin{aligned}
& {\left[-i \mathbf{p} \cdot \mathbf{q}+i \frac{\Omega \omega}{c^{2}}+\Delta \Sigma(\mathbf{q}=0)\right] M\left(\mathbf{p}, \mathbf{p}^{\prime}, \mathbf{q}\right)} \\
& \quad=\Delta g_{0}(\mathbf{q}=\mathbf{0})\left[\rho T T^{*}(\mathbf{q}=\mathbf{0})+\rho \int T\left(\mathbf{p}, \mathbf{p}_{\alpha}\right) T^{*}\left(\mathbf{p}, \mathbf{p}_{\alpha}\right) M\left(\mathbf{p}_{\alpha}, \mathbf{p}^{\prime}, \mathbf{q}\right) \mathrm{d} \mathbf{p}_{\alpha}\right] .
\end{aligned}
$$

\section{Stationary regime}

All experiments using a light source have to be performed in a dynamic way, either with a pulse of light, or with a continuous-wave (cw) source which has been switched on at a certain time. Integrating the intensity response of a medium to a pulse over all time gives a time-independent quantity. This integration is typically performed by using a detector of light with much slower dynamics than all other processes in the medium ${ }^{6}$ Switching on a cw source, typically a continuous wave laser, produces an obviously time-dependent response of an otherwise static medium. When the cw source has been on for a sufficiently long time, the response of the material becomes time-independent. These two cases, integration over time of a pulse response and limit at long time of the response of a continuous source, are equivalent and grouped under the name stationary regime. In the stationary regime, light still has a proper frequency $\omega$, but the time dependence of the intensity is neglected, $\Omega=0$.

The $\Delta g$ term can be made explicit:

$$
\begin{aligned}
2 i \Delta g & \equiv g\left(\omega^{+}, \mathbf{p}^{+}\right)-g\left(\omega^{-}, \mathbf{p}^{-}\right) \\
& =\left[\left(\frac{\omega+\Omega / 2+i \epsilon}{c}\right)^{2}-\left(\mathbf{p}+\frac{\mathbf{q}}{2}\right)^{2}\right]^{-1}-\left[\left(\frac{\omega-\Omega / 2-i \epsilon}{c}\right)^{2}-\left(\mathbf{p}-\frac{\mathbf{q}}{2}\right)^{2}\right]^{-1} \\
& =\left[\left(\frac{\omega}{c}\right)^{2}-\mathbf{p}^{2}+i \epsilon\right]^{-1}-\left[\left(\frac{\omega}{c}\right)^{2}-\mathbf{p}^{2}-i \epsilon\right]^{-1} \\
& =P V\left(\frac{\omega^{2}}{c^{2}}-\mathbf{p}^{2}\right)-i \pi \delta\left(\frac{\omega^{2}}{c^{2}}-\mathbf{p}^{2}\right)-P V\left(\frac{\omega^{2}}{c^{2}}-\mathbf{p}^{2}\right)-i \pi \delta\left(\frac{\omega^{2}}{c^{2}}-\mathbf{p}^{2}\right) \\
\Delta g & =\pi \delta\left(\mathbf{p}^{2}-\frac{\omega^{2}}{c^{2}}\right)
\end{aligned}
$$

\footnotetext{
${ }^{6}$ among others: duration of pulse, duration of light transport through the material, Brownian motion of the particles of the material, resonance time in the scatterers, etc...
} 
where, in the second line, the free space Green function is used, according to the lowdensity approximation. In the third line, the stationary case $(\Omega=0)$, and the far field approximation $(\mathbf{q} \ll \mathbf{p})$ are applied. The fourth line follows from a mathematical identity, $\lim _{\varepsilon \rightarrow 0}(x+i \varepsilon \sigma)^{-1}=P V(1 / x)-i \sigma \pi \delta(x)$ where $\sigma= \pm 1$ and $\mathrm{PV}(\mathrm{x})$ is the Cauchy principal value 7

The propagator $M\left(\mathbf{p}, \mathbf{p}^{\prime}, \mathbf{q}\right)$ is seen to be equal to the product of a delta function, and a new function: $M\left(\mathbf{p}, \mathbf{p}^{\prime}, \mathbf{q}\right) \equiv N\left(\frac{\omega}{c} \hat{\mathbf{p}}, \mathbf{p}^{\prime}, \mathbf{q}\right) \delta\left[\mathbf{p}^{2}-(\omega / c)^{2}\right]$ where $\hat{\mathbf{p}}$ is the unitary vector in the direction of $\mathbf{p}$.

The stationary Boltzmann equation for $N\left(\frac{\omega}{c} \hat{\mathbf{p}}, \mathbf{p}^{\prime}, \mathbf{q}\right)$ is

$$
\begin{aligned}
& {\left[-i \frac{\omega}{c} \hat{\mathbf{p}} \cdot \mathbf{q}+\rho \operatorname{Im} T\left(\mathbf{p}, \mathbf{p}^{\prime}\right)\right] N\left(\frac{\omega}{c} \hat{\mathbf{p}}, \mathbf{p}^{\prime}, \mathbf{q}\right)} \\
& \quad=\rho \pi\left[T T^{*}+\int\left|T\left(\frac{\omega}{c} \hat{\mathbf{p}}, \frac{\omega}{c} \hat{\mathbf{p}}_{\alpha}\right)\right|^{2} N\left(\frac{\omega}{c} \hat{\mathbf{p}}_{\alpha}, \mathbf{p}^{\prime}, \mathbf{q}\right) \frac{\omega}{c} \mathrm{~d} \hat{\mathbf{p}}_{\alpha}\right] .
\end{aligned}
$$

The physical quantities, energy density and current density of the light, are related to the averaged intensity propagator with Green functions at both ends $G\left(\mathbf{p}, \mathbf{p}^{\prime}, \mathbf{q}\right)$. The macroscopic transport is described by $\mathbf{q}$, irrespective of the internal parameters $\mathbf{p}$ and $\mathbf{p}^{\prime}$, which have to be summed. The local radiative energy and current densities are therefore [95]

$$
\begin{aligned}
W_{\omega}^{\mathrm{rad}}(\mathbf{q}) & =\left(\frac{\omega}{c}\right)^{2} \sum_{\mathbf{p}} \sum_{\mathbf{p}^{\prime}} G\left(\mathbf{p}, \mathbf{p}^{\prime}, \mathbf{q}\right) \\
\mathbf{J}_{\omega}(\mathbf{q}) & =\omega \sum_{\mathbf{p}} \sum_{\mathbf{p}^{\prime}} \mathbf{p} G\left(\mathbf{p}, \mathbf{p}^{\prime}, \mathbf{q}\right) .
\end{aligned}
$$

In order to link Eq. 2.43 to the physical quantities, $G\left(\mathbf{p}, \mathbf{p}^{\prime}, \mathbf{q}\right)$ has to be made more explicit:

$$
\begin{aligned}
\sum_{\mathbf{p}^{\prime}} G\left(\mathbf{p}, \mathbf{p}^{\prime}, \mathbf{q}\right) & =\sum_{\mathbf{p}^{\prime}} M\left(\mathbf{p}, \mathbf{p}^{\prime}, \mathbf{q}\right) g\left(\mathbf{p}_{2}\right) g^{*}\left(\mathbf{p}_{4}\right) \\
& =\sum_{\mathbf{p}^{\prime}} N\left(\frac{\omega}{c} \hat{\mathbf{p}}, \mathbf{p}^{\prime}, \mathbf{q}\right) \delta\left(\mathbf{p}^{2}-\frac{\omega^{2}}{c^{2}}\right) g\left(\mathbf{p}^{\prime}+\mathbf{q} / 2\right) g^{*}\left(\mathbf{p}^{\prime}-\mathbf{q} / 2\right) \\
& =\sum_{\mathbf{p}^{\prime}} N\left(\frac{\omega}{c} \hat{\mathbf{p}}, \mathbf{p}^{\prime}, \mathbf{q}\right) \delta\left(\mathbf{p}^{2}-\frac{\omega^{2}}{c^{2}}\right) \frac{\pi}{-i \mathbf{p}^{\prime} \cdot \mathbf{q}+\rho \operatorname{Im} T(\mathbf{p}, \mathbf{p})} \delta\left(\mathbf{p}^{\prime 2}-\frac{\omega^{2}}{c^{2}}\right) \\
& \equiv P\left(\frac{\omega}{c} \hat{\mathbf{p}}, \mathbf{q}\right) \delta\left(\mathbf{p}^{2}-\frac{\omega^{2}}{c^{2}}\right) .
\end{aligned}
$$

The newly defined function $P\left(\frac{\omega}{c} \hat{\mathbf{p}}, \mathbf{q}\right)$ follows the same Boltzmann equation as $N\left(\frac{\omega}{c} \hat{\mathbf{p}}, \mathbf{p}^{\prime}, \mathbf{q}\right)$ (Eq. 2.43 , apart from the $T T^{*}$ term which is now multiplied by a constant.

The diffusion approximation takes a last step in order to obtain the diffusion equation. The intensity propagator $P\left(\frac{\omega}{c} \hat{\mathbf{p}}, \mathbf{q}\right)$ can be expanded in moments of $\hat{\mathbf{p}}$. The first two moments correspond to the energy density $W_{\omega}^{\mathrm{rad}}(\mathbf{q})$ and the current density $\mathbf{J}_{\omega}(\mathbf{q})$. The diffusion

\footnotetext{
${ }^{7}$ The Cauchy principal value is the limit of an integral around a singularity which approaches the singularity in a symmetrical way.
} 
approximation only conserves these first two moments. The diffuse intensity is therefore almost isotropic, with a small dipole component which induces the energy flux through the medium. The intensity propagator is found to be, in the diffusion approximation,

$$
P\left(\frac{\omega}{c} \hat{\mathbf{p}}, \mathbf{q}\right)=\frac{\omega^{2}}{4 \pi c}\left[W_{\omega}^{\mathrm{rad}}(\mathbf{q})+\frac{3}{c} \hat{\mathbf{p}} \cdot \mathbf{J}_{\omega}(\mathbf{q})\right] .
$$

Filling Eq. 2.47 into the Boltzmann equation for $P\left(\frac{\omega}{c} \hat{\mathbf{p}}, \mathbf{q}\right)$ and taking its first two $\hat{\mathbf{p}}$ moments gives two equations linking the energy and current densities to each other:

$$
\left\{\begin{array}{l}
-i \mathbf{q} \cdot \mathbf{J}_{\omega}(\mathbf{q})=\text { Constant } \\
i \frac{c}{3} \mathbf{q} W_{\omega}^{\mathrm{rad}}(\mathbf{q})=\rho \int \frac{\left|T\left(\frac{\omega}{c} \hat{\mathbf{p}}_{\alpha}, \frac{\omega}{c} \hat{\mathbf{p}}\right)\right|^{2}}{(4 \pi)^{2}}\left(1-\hat{\mathbf{p}} \cdot \hat{\mathbf{p}}_{\alpha}\right) \mathrm{d} \hat{\mathbf{p}}_{\alpha} \mathbf{J}_{\omega}(\mathbf{q}),
\end{array}\right.
$$

where the optical theorem (Eq. 2.15) is used in the first equation to simplify out two terms, and in the second equation to shift the $\mathbf{J}_{\omega}$ term from lhs to rhs under the integral. A few terms have been neglected since they simplify the writing, but hardly reduce the generality of the result: all the integrals of the odd moments in $\hat{\mathbf{p}}_{\mathbf{x}}$ of the norm of the T-matrix are zero if the T-matrix is even in the transformation $\hat{\mathbf{p}}_{\mathbf{x}} \rightarrow-\hat{\mathbf{p}}_{\mathbf{x}}$.

The $\rho|T|^{2}$ term is the product of the density and the scattering cross section (see Eq. 2.15, and is therefore recognized as the inverse of the scattering mean free path. The integral over the scattering function $|T|^{2}$ and the angle $\hat{\mathbf{p}} \cdot \hat{\mathbf{p}}_{\alpha}$ is an average over the scattered angle $\langle\cos \theta\rangle$. The Boltzmann mean free path is defined as

$$
\ell_{\mathrm{B}} \equiv\left[\rho \int\left|T\left(\frac{\omega}{c} \hat{\mathbf{p}}_{\alpha}, \frac{\omega}{c} \hat{\mathbf{p}}\right)\right|^{2}\left(1-\hat{\mathbf{p}} \cdot \hat{\mathbf{p}}_{\alpha}\right) \mathrm{d} \hat{\mathbf{p}}_{\alpha}\right]^{-1}=\frac{\ell_{\mathrm{sc}}}{1-\langle\cos \theta\rangle} .
$$

Rewriting Eq. 2.48 in coordinate space,

$$
\left\{\begin{aligned}
\nabla \cdot \mathbf{J}_{\omega}(\mathbf{r}) & \sim \delta(\mathbf{r})=\text { source } \\
-\frac{c \ell_{\mathrm{B}}}{3} \nabla W_{\omega}^{\mathrm{rad}}(\mathbf{r}) & =\mathbf{J}_{\omega}(\mathbf{r})
\end{aligned}\right.
$$

and combining these two equations gives

$$
-\frac{1}{3} c \ell_{\mathrm{B}} \nabla^{2} W_{\omega}^{\mathrm{rad}}(\mathbf{r}) \sim \delta(\mathbf{r})=\text { source }
$$

which is the well-known stationary diffusion equation. The length scale associated with diffusion, i.e., to propagation of the intensity without interference, is here found to be the Boltzmann mean free path $\ell_{\mathrm{B}}$, whereas it was the scattering mean free path in the case of the field propagation. In the case of isotropic scattering, such as for point scatterers, the two mean free paths are equal, $\ell_{\mathrm{B}}=\ell_{\mathrm{sc}}$. The Boltzmann mean free path is renormalized to the transport mean free path $\ell$ in the presence of interferences. In the following sections and chapters, only $\ell$ is used, since $\ell_{\mathrm{B}}$ has no physical meaning in the case of the multiple scattering of waves. 


\section{Dynamic regime}

In a non-stationary case, the time dependence of the physical quantities $W_{\omega}^{\mathrm{rad}}$ and $\mathbf{J}_{\omega}$ has to be considered. The generalization of the previous derivation to $\Omega \neq 0$ is not straightforward $[116,117]$. In the case of light, and contrarily to electrons, the wave can be delayed in the scatterer. The assumption that the group velocity governs the propagation speed of the energy in a medium containing resonant scatterers turns out to be wrong. Following reference [95], in the dynamical case, the first moment of the Boltzmann equation (compare to Eq. 2.50 is

$$
\frac{\partial}{\partial t}\left[1+\delta_{\omega}^{(1)}\right] W_{\omega}^{\mathrm{rad}}+\nabla \cdot \mathbf{J}_{\omega}=\text { source, }
$$

where the first term of the time derivative of the energy density is the propagation delay, and the second term is the extra delay due to resonance in a scatterer. The relation $\delta_{\omega}^{(1)}=$ $\rho W_{\omega}^{\text {pot }}$ has been found following the same approximations described to obtain the stationary diffusion equation. $W_{\omega}^{\text {pot }}$ is the energy contained in the scatterer, which is not radiative, and doesn't have a current.

The diffusion equation becomes

$$
\left[\frac{\partial}{\partial t}-D \nabla^{2}\right] W_{\omega}^{\mathrm{rad}}(\mathbf{r}, t)=\text { source }
$$

where $D \equiv v_{\mathrm{E}} \ell / 3$ is the diffusion constant, $v_{\mathrm{E}}$ the energy velocity

$$
v_{\mathrm{E}}=\frac{c^{2}}{v_{\mathrm{ph}}} \frac{1}{1+\rho W_{\omega}^{\mathrm{pot}}},
$$

where $v_{\mathrm{ph}}=c / n$ is the phase velocity. The energy velocity $v_{\mathrm{E}}$ is recognized as the dynamic quantity characteristic of diffusion, as the transport mean free path $\ell$ was the stationary quantity characteristic of diffusion.

The diffusion Green function can now be explicitly written by solving the diffusion equation with a spatial and temporal Dirac delta function as source, which yields:

$$
G_{\mathrm{d}}(\mathbf{r}, t)=\frac{D}{(4 \pi D t)^{3 / 2}} \exp \left(-\frac{r^{2}}{4 D t}\right) \Theta(t)
$$

where $\Theta(t)$ is the Heaviside function, equal to 1 for $t \geq 0$, and 0 otherwise.

Integration over time of Eq. 2.55 gives the same result as solving the Green function for the stationary diffusion equation, namely

$$
G_{\mathrm{d}}(\mathbf{r})=\frac{1}{4 \pi r}
$$

Note that in the stationary diffusion, the diffusion constant does not appear, and the typical length scale must come from the boundary conditions at the interface of a diffusive material. 


\subsection{Diffusion in a slab}

In the previous section the diffusion equation (Eq. 2.53 ) for an infinite medium is derived. In order to apply such an equation to a real bounded material with an incident wave, a source for diffusion and boundary conditions have to be considered. In section 4.2 the boundary conditions are explained in more detail. The following derivations consider elastic scattering, i.e., no absorption of light.

In all the practical examples presented in this thesis, the samples are in a slab geometry, where lateral dimensions $x$ and $y$ are much larger than transverse dimension $z$. The system is therefore modeled as a medium in which the diffusion equation holds, infinite in the $x$ and $y$ directions, bounded at $z=0$ and $z=L$, with vacuum at both sides. The incident plane wave originates from $z=-\infty$.

The source of light, such as an incident plane wave, is rigorously described within the framework of the multiple scattering theory, presented in the previous section. Within the diffusion approximation, only diffuse light can be handled, and therefore an incident plane wave can not be inserted as source in the diffusion equation. According to Eq. 2.24, an incident plane wave decays exponentially with the depth, in units of the extinction mean free path. In the case of elastic scattering, all the light which has left the coherent beam has scattered once. If the scattering is isotropic, like for point scatterers or Rayleigh scattering, the light which has left the coherent beam is already within the diffusion approximation. The source of diffuse light is therefore

$$
S_{\mathrm{d}}(z)=-\frac{\partial I_{\mathrm{coh}}}{\partial z}=\frac{I_{0}}{\ell_{\mathrm{sc}}} \exp \left(-\frac{z}{\ell_{\mathrm{sc}}}\right) .
$$

If the scattering is anisotropic, for example for Mie spheres [4] or liquid crystals [120], light which has been scattered only once is not yet part of the diffuse intensity. The transport mean free path is the average distance after which light gets diffuse, i.e., when the distribution of light is (almost) isotropic. The phenomenological way [121] of treating the source in the case of anisotropic scattering is therefore to set the source at exactly one transport mean free path from the incident interface, inside the slab: $S_{\mathrm{d}}(z)=S_{0} \delta(z-\ell)$. A natural although still phenomenological extension [122] to the previous description considers a source which exponentially decreases with depth, in units of the transport mean free path $S_{\mathrm{d}}(z)=S_{0} \exp (-z / \ell)$. The source found for isotropic scattering links to this exponential source, which justifies the use of the exponential for at least the small values of $\langle\cos \theta\rangle$ when $\ell$ is close to $\ell_{\mathrm{sc}}$.

\subsubsection{Stationary diffusion}

The following boundary conditions, Eqs. 4.7 and 4.9 from section 4.2, are taken:

$$
\begin{aligned}
& W_{\omega}^{\mathrm{rad}}(z)-z_{\mathrm{e}_{1}} \frac{\partial W_{\omega}^{\mathrm{rad}}(z)}{\partial z}=0 \quad \text { at } \quad z=0 \\
& W_{\omega}^{\mathrm{rad}}(z)+z_{\mathrm{e}_{2}} \frac{\partial W_{\omega}^{\mathrm{rad}}(z)}{\partial z}=0 \quad \text { at } \quad z=L,
\end{aligned}
$$

where $z_{\mathrm{e}}$, the extrapolation length, is of the order of the transport mean free path, and is a property of the interface, potentially different for the front and back interfaces. 


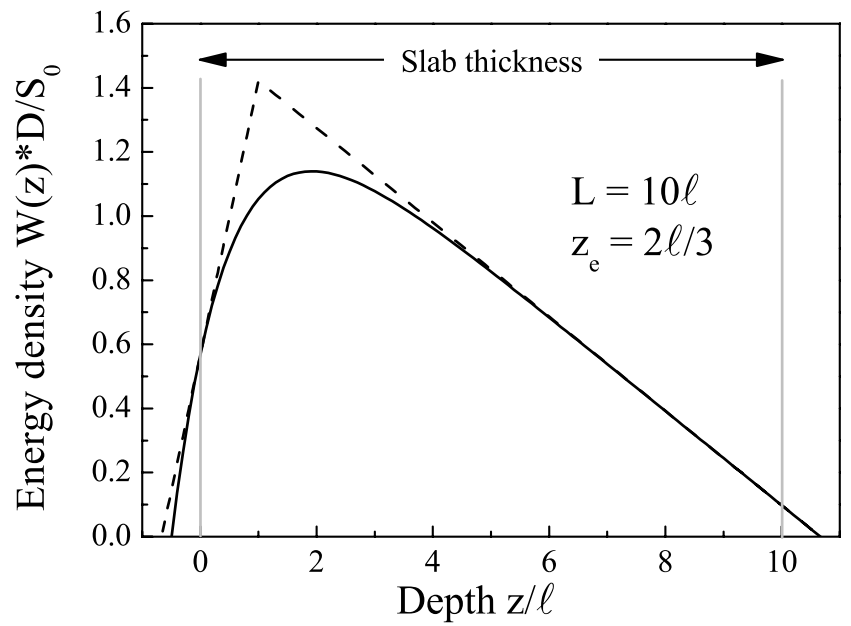

Figure 2.2: Normalized energy density $W_{\omega}^{\mathrm{rad}}(z)$ as a function of depth inside a slab of thickness $L=10 \ell$, and where $z_{\mathrm{e}_{1}}=z_{\mathrm{e}_{2}}=2 \ell / 3$. The full curve is the solution to the diffusion equation with an exponential source (Eq.2.61). The dashed curve is the solution for a delta source at $z=\ell(E q \cdot 2.60)$. The gray lines symbolizes the boundaries of the slab.

Solving the stationary diffusion equation with these boundary conditions and for a delta source is very easy. In the ranges $[0 ; \ell[$ and $] \ell ; L]$ separately, the intensity $W_{\omega}^{\mathrm{rad}}(z)$ is a linear function of the depth, the integration constants are found:

$$
W_{\omega}^{\mathrm{rad}}(z)=\frac{S_{0}}{D} \begin{cases}\frac{L-\ell+z_{\mathrm{e}_{2}}}{L+z_{\mathrm{e}_{1}}+z_{\mathrm{e}_{2}}}\left(z+z_{\mathrm{e}_{1}}\right), & 0 \leq z \leq \ell \\ \frac{\ell+z_{\mathrm{e}_{1}}}{L+z_{\mathrm{e}_{1}}+z_{\mathrm{e}_{2}}}\left(L+z_{\mathrm{e}_{2}}-z\right), & \ell \leq z \leq L .\end{cases}
$$

Integrating the diffusion equation over an exponential source gives, for the whole slab,

$$
W_{\omega}^{r a d}(z)=\frac{S_{0}}{D}\left[\frac{\ell+z_{\mathrm{e}_{1}}}{L+z_{\mathrm{e}_{1}}+z_{\mathrm{e}_{2}}}\left(L+z_{\mathrm{e}_{2}}-z\right)-\ell \exp \left(-\frac{z}{\ell}\right)\right],
$$

where the thickness $\mathrm{L}$ of the slab is considered much bigger than the transport mean free path, and thus terms as $\exp (-L / \ell)$ are neglected. Both solutions to the diffusion equation in a slab, for exponential and delta sources, are plotted in Fig. 2.2.

The first measurement which can be done on a slab of diffusive material is the so-called total transmission. The coherent transmission, i.e., the remaining of the incident plane wave, has already been specified in Eq. 2.24, and seen to be a function of the scattering mean free path. The transmission integrated over all angles is called the total transmission. The total transmission $T_{\mathrm{d}}$ is defined as the total flux at $z=L$ divided by the incident flux $S_{0}$, and is found to be

$$
T_{\mathrm{d}} \equiv \frac{\mathbf{J}_{\omega}(L) \cdot \hat{\mathbf{z}}}{S_{0}}=-\left.\frac{D}{S_{0}} \frac{\partial W_{\omega}^{\mathrm{rad}}}{\partial z}\right|_{z=L}=\frac{\ell+z_{\mathrm{e}_{1}}}{L+z_{\mathrm{e}_{1}}+z_{\mathrm{e}_{2}}},
$$


where the coherent beam contribution has been left out. For all but very thin samples (a few $\ell$ thick) this approximation is valid. It is possible to add the coherent beam contribution, although the validity of the diffusion equation with boundary conditions breaks for very thin samples.

The total transmission scales with the inverse of the thickness, and can be recognized as the equivalent of Ohm's law for the conductance in electronic systems.

In the same way as for transmission, the total reflection can be calculated. The total reflection $R_{\mathrm{d}}$ is defined as the flux going back through the incident interface, at $z=0$, normalized to the incident flux. The reflection $R_{\mathrm{d}}$ is found to be complementary to $T_{\mathrm{d}}$, as necessary:

$$
R_{\mathrm{d}} \equiv \frac{\mathbf{J}_{\omega}(0) \cdot(-\hat{\mathbf{z}})}{S_{0}}=\frac{L-\ell+z_{\mathrm{e}_{2}}}{L+z_{\mathrm{e}_{1}}+z_{\mathrm{e}_{2}}}=1-T_{\mathrm{d}}
$$

If absorption dominates in the medium, i.e., the diffuse absorption length $L_{\mathrm{a}} \ll \ell$, the total transmission becomes exponentially decreasing with the thickness [11]: $T_{\mathrm{d}}\left(L_{\mathrm{a}} \ll \ell\right) \propto \exp \left(-L / L_{\mathrm{a}}\right)$. In the same limit, the diffuse reflection does not depend on the thickness anymore, and decreases along with the diffuse absorption length.

\subsubsection{Dynamic diffusion}

Solving the time-dependent diffusion equation (Eq. 2.53) with the mixed boundary conditions Eqs. 2.58 and 2.59 is not possible in closed form [123]. Instead, the Dirichlet boundary conditions (Eq. 4.10, which are equivalent to the mixed conditions for small enough absorbtion, are used:

$$
W_{\omega}^{\mathrm{rad}}(z)=0 \text { at }\left\{\begin{array}{l}
z=-z_{\mathrm{e}_{1}} \\
z=L+z_{\mathrm{e}_{2}} .
\end{array}\right.
$$

The solutions to the time-dependent diffusion equation, Eq. 2.53, are the product of a space harmonic and a time exponential. Considering a delta source located at $z=\ell$ and $t=0$, the energy density in time and space is found to be

$$
W^{r a d}(z, t)=\sum_{m=1}^{\infty} \exp \left[\frac{-\pi^{2} m^{2} D t}{L_{\mathrm{ex}}^{2}}\right] \sin \left(\pi m \frac{z+z_{\mathrm{e}_{1}}}{L_{\mathrm{ex}}}\right) \frac{I_{0} \sin \left(\pi m \frac{\ell+z_{\mathrm{e}_{1}}}{L_{\mathrm{ex}}}\right)}{L_{\mathrm{ex}}} \Theta(t),
$$

where $I_{0} / L_{\mathrm{ex}}$ is the intensity of the source and $L_{\mathrm{ex}} \equiv L+z_{\mathrm{e}_{1}}+z_{\mathrm{e}_{2}}$ is a shorthand, sometimes (abusively) called the effective thickness, and which is here called extrapolated thickness.

The first sine term in Eq. 2.65 is the $m^{\text {th }}$ eigenmode of the slab for the stationary diffusion equation, with the boundary conditions of Eq. 2.64 The exponential term in Eq. 2.65. coming from the dynamic diffusion equation, rules how each eigenmode decay in time. Low order eigenmodes (for small $\mathrm{m}$ ) vary slowly in space and decay slowly because of diffusion. Higher order eigenmodes vary fast in space and are the first ones to disappear because of diffusion. The last sine term in Eq. 2.65 is the decomposition of the spatial delta source in harmonics. The Heaviside function allows a light density inside the slab only after the temporal delta source at $t=0$. 
Calculating the flux at the second interface leads to the normalized time-dependent (total) diffuse transmission [11,28, 124]

$$
T_{\mathrm{d}}(t)=-\frac{\pi D}{L_{\mathrm{ex}}^{2}} \sum_{m=1}^{\infty} m \exp \left[\frac{-\pi^{2} m^{2} D t}{L_{\mathrm{ex}}^{2}}\right] \cos \left(\pi m \frac{L+z_{\mathrm{e}_{1}}}{L_{\mathrm{ex}}}\right) \sin \left(\pi m \frac{\ell+z_{\mathrm{e}_{1}}}{L_{\mathrm{ex}}}\right) \Theta(t) .
$$

Multiple scattering of light produces paths for light of very different length inside the sample. Light which is scattered mostly forward follows the shortest optical path through the slab, and therefore is transmitted first. Light which is scattered a lot and performs a random walk before reaching the back interface follows a much longer optical path, and is transmitted much later. Multiple scattering of light therefore spreads the initial pulse of light in time, depending on the diffusion constant.

The behavior at long time of Eq. 2.66 is dominated by the least decaying exponential, for $m=1$, giving the time tail with decay rate $\Gamma \equiv \pi^{2} D / L_{\mathrm{ex}}^{2}$,

$$
\frac{I_{\mathrm{d}}(t \rightarrow \infty)}{I_{0}} \propto \exp (-\Gamma t) .
$$

The proportionality constant is easily found from the term $m=1$ in Eq. 2.66

\subsection{Enhanced backscattering}

\subsubsection{Derivation of the EBS line shape}

In section 2.4 the Boltzmann and diffusion equations are derived. In order to obtain this result which is free of interference, the intensity propagator had to be limited to only the ladder contribution (see Eqs. 2.26 and 2.30). The following chapter focusses on the first effect of interference present in ensemble-averaged multiple scattering of light. The sum of most-crossed diagrams (Eq. 2.31), in addition to the ladder contribution, is retained: $\langle R\rangle=\langle L\rangle+\langle C\rangle$. Otherwise, the same approximations are made as in the previous sections, so that the calculation of the Ladder contribution itself remains true, i.e., following the diffusion equation, Eq. 2.53 .

For the derivation of the EBS in this section, point scatterers are considered. The summation of the ladder terms in space coordinates, and for point scatterers $t\left(\mathbf{r}^{\prime}, \mathbf{r}, \omega\right)=\delta\left(\mathbf{r}^{\prime}-\mathbf{R}\right) \delta(\mathbf{r}-\mathbf{R}) t(\omega)$ (from Eq. 2.13), can be written 8

$$
\begin{aligned}
\left\langle L\left(\mathbf{r}_{1}, \mathbf{r}_{2}, \mathbf{r}_{3}, \mathbf{r}_{4}\right)\right\rangle=\delta\left(\mathbf{r}_{1}-\mathbf{r}_{3}\right)\left[\rho|t(\omega)|^{2}\right. & \delta\left(\mathbf{r}_{1}-\mathbf{r}_{2}\right) \delta\left(\mathbf{r}_{1}-\mathbf{r}_{\mathbf{4}}\right) \\
& \left.+F\left(\mathbf{r}_{1}, \mathbf{r}_{2}\right) \delta\left(\mathbf{r}_{2}-\mathbf{r}_{4}\right)\right] .
\end{aligned}
$$

The ladder sum is here described as the first order scattering, explicitly written in the case of point scatterers, and the higher orders, summed in $F\left(\mathbf{r}_{1}, \mathbf{r}_{2}\right)$, where the incoming and outgoing space coordinates have been factorized out. This factorization is only possible in the case of point scatterers, where the T-matrix is proportional to a Dirac delta function of the position of the scatterer. The intensity Green function in the slab $F\left(\mathbf{r}_{1}, \mathbf{r}_{2}\right)$ is made explicit later on.

\footnotetext{
${ }^{8}$ The original Ansatz was presented by Tsang and Ishimaru [125-127].
} 
The solution for the most-crossed diagrams is very easy thanks to a trick. By taking the most-crossed diagrams of Eq. 2.31 and rotating the bottom line by $180^{\circ}$, the ladder diagrams are recognized, with the exception of the first order term. This rotation implies that $\langle C\rangle$ is equal to $\langle L\rangle$, minus the first order term, where bottom coordinates $\mathbf{r}_{\mathbf{3}}$ and $\mathbf{r}_{\mathbf{4}}$ are swapped:

$$
\left\langle C\left(\mathbf{r}_{1}, \mathbf{r}_{2}, \mathbf{r}_{3}, \mathbf{r}_{4}\right)\right\rangle=F\left(\mathbf{r}_{1}, \mathbf{r}_{2}\right) \delta\left(\mathbf{r}_{1}-\mathbf{r}_{4}\right) \delta\left(\mathbf{r}_{2}-\mathbf{r}_{3}\right) .
$$

The physical meaning of this trick to solve for $\langle C\rangle$ is time symmetry. A light path and its time reverse have the same optical length. If the origin and the end of a path are at the same location, and if the time reverse of the path is distinct from itself, then the two fields, along each path, will interfere constructively at the origin. Constructive interference means having twice as much intensity than would have been measured without interference. The Green function $\langle C\rangle$ has here the role of doubling the intensity where time reversed paths interfere.

According to Eq. 2.27, for the intensity of light to be calculated, the incident field, the averaged Green function and the intensity propagator $\langle R\rangle$ have to be known.

The incident field in the medium is the coherent beam:

$$
\left\langle\psi_{\text {inc }}\left(\mathbf{r}_{1}\right)\right\rangle=\sqrt{I_{0}} \exp \left(i \mathbf{K}_{\mathrm{i}} \cdot \mathbf{r}_{1}\right),
$$

where $\mathbf{K}_{\mathrm{i}} \approx \mathbf{k}_{\mathrm{i}}+i \hat{\mathbf{z}} /\left(2 \ell_{\mathrm{sc}} \mu_{\mathrm{i}}\right)$ and $\mathbf{k}_{\mathrm{i}}$ is the wave vector of the incident field on the medium. The factor $\mu_{\mathrm{i}} \equiv \cos \theta_{\mathrm{i}}$ takes the incident angle into account. The exponential decrease in depth is faster at a higher incident angle, and leads to the term $\ell_{\mathrm{sc}} \mu_{\mathrm{i}}$ for the extinction of the incident beam. The intensity of the incident wave is $I_{0}$.

Knowing the field Green function in free space (Eq. 2.5) and in the material (Eq. 2.23), the Green function for backscattering $g_{\mathrm{b}}$, where $\mathbf{r}_{\mathbf{1}}$ is far before the slab and $\mathbf{r}_{\mathbf{2}}$ inside, is $[96,97]$

$$
\left\langle g_{\mathrm{b}}\left(\mathbf{r}_{1}, \mathbf{r}_{2}\right)\right\rangle \approx-\frac{\exp \left(-i k_{0} r_{1}\right)}{4 \pi r} \exp \left(i \mathbf{K}_{\mathrm{b}} \cdot \mathbf{r}_{2}\right)
$$

where $r_{2} \ll r_{1}, \mathbf{K}_{\mathrm{b}} \approx \mathbf{k}_{\mathrm{s}}+i \hat{\mathbf{z}} /\left(2 \ell_{\mathrm{sc}} \mu_{\mathrm{s}}\right)$ and $\mathbf{k}_{\mathrm{s}}$ is the wave vector of the field scattered out of the medium. The scattered angle is taken into account by $\mu_{\mathrm{s}} \equiv \cos \theta_{\mathrm{s}}$, equivalently as for the incident field above.

The bistatic coefficient $\gamma\left(\mu_{\mathrm{i}}, \mu_{\mathrm{s}}\right)$ is used to describe the scattered intensity in a normalized way. The coefficient is calculated for given incident and scattered directions, for a normalized incident intensity, corrected for the distance to the scattering medium and the observed area $A \mu_{\mathrm{i}}$ :

$$
\gamma\left(\mu_{\mathrm{i}}, \mu_{\mathrm{s}}\right) \equiv \frac{4 \pi r^{2}}{I_{0} A \mu_{\mathrm{i}}}\langle I(\mathbf{r})\rangle \equiv \gamma_{\mathrm{s}}\left(\mu_{\mathrm{i}}, \mu_{\mathrm{s}}\right)+\gamma_{\ell}\left(\mu_{\mathrm{i}}, \mu_{\mathrm{s}}\right)+\gamma_{\mathrm{c}}\left(\mu_{\mathrm{i}}, \mu_{\mathrm{s}}\right),
$$

where the bistatic coefficient has been split into three parts corresponding respectively to the single scattering (first term in Eq. 2.68), the rest of the ladder contribution (second term in Eq. 2.68, and the most-crossed diagrams (in Eq. 2.69).

Using the definition of the bistatic coefficients (Eq. 2.72), the intensity as a function of the intensity propagator $\langle R\rangle$ (Eq. 2.27), the approximation $\langle R\rangle=\langle L\rangle+\langle C\rangle$, the expressions 
for $\langle L\rangle$ and $\langle C\rangle$ for point scatterers (Eqs. 2.68 and 2.69, the incident field in Eq. 2.70 and the backscattering Green function in Eq. 2.71, the following equations are found:

$$
\begin{gathered}
\gamma_{\mathrm{s}}\left(\mu_{\mathrm{i}}, \mu_{\mathrm{s}}\right)=\frac{\mu_{\mathrm{s}}}{\mu_{\mathrm{s}}+\mu_{\mathrm{i}}}\left[1-\exp \left(-\frac{L}{\ell} 2 v\right)\right] \\
\gamma_{\ell}\left(\mu_{\mathrm{i}}, \mu_{\mathrm{s}}\right)=\frac{1}{4 \pi \mu_{\mathrm{i}}} \int_{0}^{L} \int_{0}^{L} F\left(z_{1}, z_{2}, \mathbf{q}_{\perp}=0\right) \exp \left[-\frac{1}{\ell}\left(\frac{z_{1}}{\mu_{\mathrm{s}}}+\frac{z_{2}}{\mu_{\mathrm{i}}}\right)\right] \mathrm{d} z_{1} \mathrm{~d} z_{2} \\
\gamma_{\mathrm{c}}\left(\mu_{\mathrm{i}}, \mu_{\mathrm{s}}\right)=\frac{1}{4 \pi \mu_{\mathrm{i}}} \int_{0}^{L} \int_{0}^{L} F\left(z_{1}, z_{2}, \mathbf{q}_{\perp}\right) \cos \left[\frac{u}{\ell}\left(z_{1}-z_{2}\right)\right] \\
\times \exp \left[-\frac{v}{\ell}\left(z_{1}+z_{2}\right)\right] \mathrm{d} z_{1} \mathrm{~d} z_{2},
\end{gathered}
$$

where the shorthands $u, v$ and $\alpha$ are defined as

$$
u \equiv k_{0} \ell\left(\mu_{\mathrm{i}}-\mu_{\mathrm{s}}\right), \quad v \equiv \frac{1}{2}\left(\frac{1}{\mu_{\mathrm{s}}}+\frac{1}{\mu_{\mathrm{i}}}\right), \quad \alpha \equiv q_{\perp} \ell,
$$

and where the Green function $F\left(\mathbf{r}_{1}, \mathbf{r}_{2}\right)$ is Fourier-transformed on the $x$ and $y$ components only, to take advantage of the translation invariance along those two directions:

$$
F\left(z, \mathbf{q}_{\perp}\right) \equiv \int F\left(z, \mathbf{r}_{\perp}\right) \exp \left(-i \mathbf{r}_{\perp} \cdot \mathbf{q}_{\perp}\right) \mathrm{d} \mathbf{r}_{\perp} .
$$

The coefficient $\gamma_{\mathrm{s}}$ corresponds to light paths which only scatter once. The EBS cone $\gamma_{\mathrm{c}}$ does not have any contribution from the single scattering. Indeed, the expected factor 2 in intensity at exact backscattering compared to the case of diffusion without interference originates from distinct time-reversed paths. At exact backscattering, where $\mathbf{k}_{\mathrm{s}}=-\mathbf{k}_{\mathrm{i}}$, the path which scatters only once is its own time reverse. The constructive interference which leads to the EBS does not happen for single scattering. Without taking into account the single scattering, or any breaking of time symmetry, the enhancement factor has to be 2 , because of fully constructive interference.

In order to proceed with the integration of Eqs. 2.74 and 2.75, the intensity Green function for the slab, $F\left(\mathbf{r}_{1}, \mathbf{r}_{2}\right)$ has to be found. In an infinite medium, this Green function is described by the diffusion equation, and therefore is $G_{\mathrm{d}}(\mathbf{r})$ in Eq. 2.56

The description of diffusion in a bounded material requires boundary conditions, like the mixed conditions of Eqs. 2.58 and 2.59 or Dirichlet conditions, Eq. 2.64. The case of a semi-infinite diffusive material is considered. Only the interface at $z=0$ is retained, which requires only one boundary condition. The Green function for the semi-infinite case with transparent interface is calculated from the infinite-medium Green function $G_{\mathrm{d}}$ by subtracting the mirror image of $G_{\mathrm{d}}$ from a trapping plane [99], like $G_{\mathrm{d}}\left(z_{1}-z_{2}\right)-G_{\mathrm{d}}\left(z_{1}+z_{2}+2 z_{\mathrm{e}}\right)$. The trapping plane is where the energy density is extrapolated to 0 , at one extrapolation length $z_{\mathrm{e}}$ outside the medium. This mirroring derives from the Dirichlet boundary condition of Eq. 2.64. In the case of a finite reflectivity at the interface, the mixed boundary condition Eq. 2.58 is taken. The mirror image is taken from the interface itself and weighted by the fresnel reflection coefficient, which, following reference [98], is

$$
F\left(z_{1}, z_{2}, \mathbf{q}_{\perp}\right)=G_{\mathrm{d}}\left(z_{1}-z_{2}, \mathbf{q}_{\perp}\right)+\frac{z_{\mathrm{e}} q_{\perp}-1}{z_{\mathrm{e}} q_{\perp}+1} G_{\mathrm{d}}\left(z_{1}+z_{2}, \mathbf{q}_{\perp}\right) .
$$


The diffusion Green function has been found in the previous section, Eq. 2.56, and can now be Fourier transformed along the $x$ and $y$ coordinates (see Eq. 2.77) as

$$
G_{\mathrm{d}}\left(z, \mathbf{q}_{\perp}\right)=\frac{\exp \left(-z q_{\perp}\right)}{2 q_{\perp}} .
$$

After the introduction of the Green function for the semi-infinite medium, Eq. 2.78 into the bistatic coefficients, Eqs. 2.74 and 2.75 and the straightforward integration, the coefficients read

$$
\begin{aligned}
& \gamma_{\ell}\left(\mu_{\mathrm{i}}, \mu_{\mathrm{s}}\right)=3 \mu_{\mathrm{s}}\left(\tau_{\mathrm{e}}+\frac{\mu_{\mathrm{s}} \mu_{\mathrm{i}}}{\mu_{\mathrm{s}}+\mu_{\mathrm{i}}}\right) \\
& \gamma_{\mathrm{c}}\left(\mu_{\mathrm{i}}, \mu_{\mathrm{s}}\right)=\frac{3}{2 \mu_{\mathrm{i}} v} \frac{1}{(\alpha+v)^{2}+u^{2}}\left(1+\frac{2 v \tau_{\mathrm{e}}}{1+\tau_{\mathrm{e}} \alpha}\right),
\end{aligned}
$$

where $z_{\mathrm{e}} \equiv \tau_{\mathrm{e}} \ell$ and the other shorthands were defined in Eq. 2.76

\subsubsection{Physics behind the EBS line shape}

Eq. 2.81 for the line shape of the EBS reduces to the well-known result $[96,121]$ for $\tau_{\mathrm{e}}=2 / 3$. Indeed, in the absence of reflection at the interface, using the mixed boundary condition at the interface (Eq. 2.58) is equivalent to taking a trapping plane, or Dirichlet condition, at an extrapolation length distance from the interface (Eq. 2.64).

The generalization of the EBS line shape to an interface with internal reflection has been initially presented by Lagendijk et al. [98], and popularized by Zhu et al. [128], although the first detailed and almost exact derivation was given by Den Outer [97]. A slight error in this last work prevented a fully satisfying result, since the EBS cone in the absence of single scattering was found to have an enhancement factor bigger than 2 for the reflecting interface. The derivation presented here, which leads to Eqs. 2.80 and 2.81. retain the enhancement factor of 2 when the single scattering contribution is neglected. Exact backscattering is given by equal incident and scattered angle, $\theta_{\mathrm{i}}=\theta_{\mathrm{s}}$, and for $\alpha=0$.

$$
\gamma_{\ell}\left(\mu_{\mathrm{i}}=\mu_{\mathrm{s}} \equiv \mu, \alpha=0\right)=\frac{3}{2} \mu\left(\mu+2 \tau_{\mathrm{e}}\right)=\gamma_{\mathrm{c}}\left(\mu_{\mathrm{i}}=\mu_{\mathrm{s}} \equiv \mu, \alpha=0\right) .
$$

Fig. 2.3 shows the line shape of EBS, normalized to $\gamma_{\ell}(\mu=0, \alpha=0)$, for a given mean free path $\left(k_{0} \ell=10\right)$ and for different reflectivity coefficients $(\bar{R}=0$ up to $\bar{R}=0.8)$.

It had been concluded by Den Outer [97] that an internal reflectivity $\bar{R}$ decreased the width of the EBS cone as $\theta_{\text {FWHM }} \rightarrow \theta_{\text {FWHM }}(1-\bar{R})$. However, as can be seen from the right inset of Fig. 2.3, the EBS width is not exactly linear in $\bar{R}$. A better description can be seen from the left inset of Fig. 2.3, where the inverse EBS width is found practically linear in the extrapolation ratio $\tau_{\mathrm{e}}$. According to the exact derivation, the transport mean free path is related to the EBS width and the internal reflectivity coefficient as

$$
\ell \approx \frac{\lambda_{0}}{2 \pi} \frac{0.7}{\theta_{\mathrm{FWHM}}} /\left[1+\left(\tau_{\mathrm{e}}-2 / 3\right) \times 0.355\right] .
$$

Apart from the width of the EBS cone, information can be found from its top and from the enhancement factor. The enhancement factor $E$ is defined as the intensity at exact 


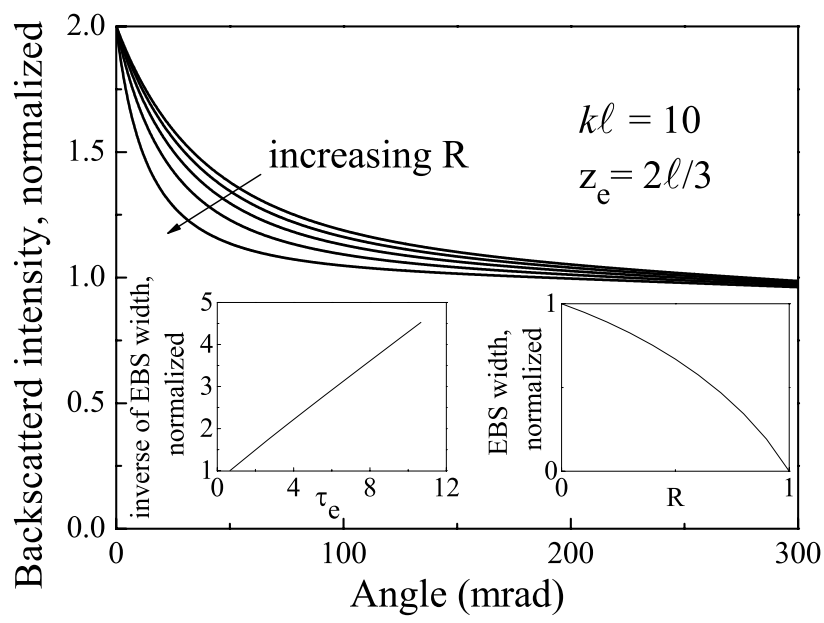

Figure 2.3: Calculated line shapes of EBS for $k_{0} \ell=10$ and different reflectivity coefficients $\bar{R}$. From top to bottom curve, $R=0,0.2,0.4,0.6,0.8$. Left inset, inverse of calculated full width at half maximum, $1 / \theta_{\mathrm{FWHM}}$, normalized to 1 for $\tau_{\mathrm{e}}=2 / 3$ versus extrapolation ratio $\tau_{\mathrm{e}}$. Right inset, $\theta_{\mathrm{FWHM}}$, normalized to $\bar{R}=0$ versus the internal reflectivity $\bar{R}$.

backscattering normalized to the intensity without interference at backscattering. $E$ reaches the theoretical value of 2 only when time reversal of all light paths which contribute to the backscattering is preserved [17]. Experimental artifacts [97, 129] can reduce the enhancement factor by preventing symmetrical time-reversed paths to be detected. From Eq. 2.73. the single scattering is found to reduce the enhancement factor because of the lack of distinct time reverse (see also [96]). The single scattering contribution can be completely filtered out by using the helicity-preserving channel [130], i.e., when the incident and scattered polarizations are circular and equal? If a light path inside the sample contains recurrent scattering, i.e., loops, a distinct time reverse is also not present, irremediably reducing the enhancement factor from stronger scattering samples [131]. Time symmetry can be broken by use of a magnetic field thanks to the Faraday effect [132,133], thereby reducing the enhancement factor at will. Brownian motion, if quick enough, implies that the scatterers are not in the same configuration for a path and its time reverse. Macroscopic systems, as well as cold atoms, are far from displaying a Brownian motion quick enough to effectively break time symmetry. Extra effects such as absorption, finite size of the medium, and localization, which impose a limit on the path length distribution, do not break the time symmetry and therefore do not reduce the enhancement factor. Absorption and finite size can be included in the derivation of the EBS line shape [96,121]. Both effects cut off the long light paths contributing to the EBS cone, therefore rounding its top. The amount of rounding can be qualitatively described by the angle $\Delta \Theta_{R}$ at which the line shape differs from the the triangular shape of Eq.2.81. In presence of absorption with diffuse absorption

\footnotetext{
${ }^{9}$ The backscattering off a mirror symmetrizes the incident circular polarization. Seen behind helicity preserving polarizers, a mirror, or in this case a single scattering in the backward direction, remains black.
} 
length $L_{\mathrm{a}}$ and for a slab of thickness $L$, this rounding angle $\Delta \theta_{\mathrm{R}}$ is found to be $[83,96]$

$$
\Delta \theta_{\mathrm{R}}=\frac{1}{k_{0} L_{\mathrm{a}}} \operatorname{coth}\left(\frac{L_{\mathrm{ex}}}{L_{\mathrm{a}}}\right) .
$$

If the absorption is negligible, $L_{\mathrm{a}} \gg L_{\mathrm{ex}}$, the rounding is linear in the inverse thickness, $\Delta \theta_{\mathrm{R}}=1 /\left(k_{0} L_{\mathrm{ex}}\right)$. If the absorption is predominant, $L_{\mathrm{a}} \ll L_{\mathrm{ex}}$, the rounding is constant, $\Delta \theta_{\mathrm{R}}=1 /\left(k_{0} L_{\mathrm{a}}\right)$.

Localization of light also modifies the distribution of path lengths, and is expected to change the top of the EBS cone. According to the scaling theory of localization, the top of the EBS cone [134-136] gets more rounded but only looses its cusp passed the localization transition.

\subsection{Conclusions}

According to multiple-scattering theory, the strong interaction of light with an infinite, inhomogeneous and disordered material leads to the diffusion equation, provided interference effects are neglected and successive scattering events are independent. Within the diffusion regime, a stationary measurement depends on the transport mean free path, whereas a dynamic measurement depends on the diffusion constant. Interference effects in ensembleaveraged independent multiple scattering amount to the EBS, an increase in intensity reflected around the backscattered direction. The main information from an EBS cone is extracted from its width, which is related to the transport mean free path. Additional information can be gained from the careful analysis of the top of the EBS cone, such as finite thickness, absorption, or localization. 



\section{Chapter 3}

\section{Chemistry of porous gallium phosphide}

\subsection{Introduction}

Anderson localization of light necessitates a strongly-scattering disordered material. Strong scattering occurs in inhomogeneous materials where the variations in refractive index are large, and the spatial range of the variations is close to the wavelength of light. Some of the strongest scattering materials are powdered semiconductors, i.e., aggregations of small particles. Powders of titanium dioxide [72,73], zinc oxide [74,75], gallium arsenide [76], germanium [77-79] and silicon ( $\mathrm{Si}$ ) [80,81] have all been shown to scatter light very strongly at visible or near-infrared wavelengths. The semiconductor with the highest refractive index in the visible, and therefore the largest chance to strongly scatter visible light, is gallium phosphide $(\mathrm{GaP})$. The band gap energy of $\mathrm{GaP}$ is $2.24 \mathrm{eV}$ [137], corresponding to a wavelength of $550 \mathrm{~nm}$. The refractive index of $\mathrm{GaP}$ at $633 \mathrm{~nm}$ is 3.3 [137]. A strongly scattering material can be made from GaP by grinding the single crystal into small particles. Such grinding procedure has been suspected and shown to introduce extra absorption compared to the single-crystalline semiconductor $[77,80,138]$. An alternative to grinding, and therefore powdered semiconductors, is the formation of pores inside the single crystal. Various semiconductors have been made porous [139-141] by electrochemical etching ${ }^{1}$ In chemical etching, a metal or semiconductor dissolves by direct transfer of valence electrons to an etching agent in solution. In electrochemical etching, a voltage source and counter electrode are used: by fixing the electrochemical potential of the sample, valence electrons can be removed via the external circuit and the sample dissolves. The etching of Si is a very active line of research, and a lot of different parameters modifying the porous structure in Si have been studied: doping concentration [142, 143], etching potential [143, 144], temperature [145, 146], electrolyte [142,144] and magnetic field [147]. GaP has been initially made porous by galvanostati ${ }^{2}$ etching in hydrofluoric acid $[148,149]$ and at constant potential in sulfuric acid $[85,87]$. During etching of GaP, the material becomes porous and

\footnotetext{
${ }^{1}$ Equivalently called anodic etching.

${ }^{2} \mathrm{~A}$ galvanostatic etching imposes a constant current instead of a constant potential.
} 


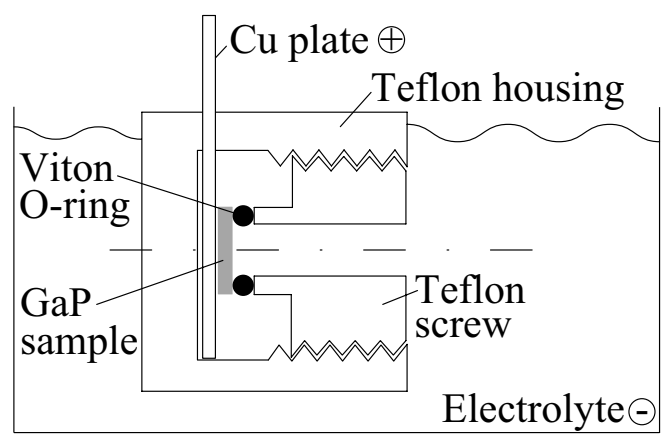

Figure 3.1: Cartoon of the anodic etching setup. The GaP sample is connected through a conductive paste to the copper plate, serving as anode, i.e., positively charged compared to the electrolyte. The electrolyte is in contact with the other side of the sample only, thanks to the Viton $O$ ring pressed between the sample and the Teflon housing.

turns from a transparent orange-brown to a diffusive yellow. A strong specular reflection can still be observed from the etched surface, due to a non-porous GaP top-layer. This highly-reflective top-layer influences greatly the stationary diffusion experiments (see in particular section 4.3.2). Photochemical etching, coupling the effect of an acid with the absorption of light in the semiconductor, allows the selective removal of the top-layer. A further increase of the size of the pores can be achieved by chemical etching, to broaden the range of properties of strongly scattering samples. The current chapter is the prolongation of, and inspired by, a previous study [81].

In the following section, the current understanding of the electrochemical etching of $\mathrm{GaP}$ is reviewed. Several important parameters, among which the dopant density of the $\mathrm{GaP}$ wafer, the etching reagent and the temperature, are studied and presented. In section 3.3 , the two extra processes, photochemical and chemical etching, are described and commented on.

\subsection{Electrochemical etching of GaP}

Electrochemical etching with well-chosen etching conditions allows the formation of a mechanically-stable porous layer inside the $\mathrm{GaP}$ wafer [85, 87]. For all experiments presented in this section, the following setup, depicted in Fig. 3.1. is used. GaP wafers doped with sulfut ${ }^{3}$ are commercially available ${ }^{4}$. Polished wafers of doping concentration ${ }^{5}$ $N=(0.5,5,6,7,15 \pm 5) \times 10^{17} \mathrm{~cm}^{-3}$ and (100)-oriented surface were used. The thickness of the wafers is typically around $300 \mu \mathrm{m}$. A piece of $\mathrm{GaP}$ is cleaved or diced from a wafer, to obtain a sample of area around $1 \mathrm{~cm}^{2}$. The sample is then glued on a copper plate with a conductive silver epoxy. The copper plate and the sample are enclosed in a Teflon housing, leaving a circular opening of $5.5 \mathrm{~mm}$ in diameter in the front face of the sample. A thick Viton ${ }^{6}$ O-ring is pressed between the GaP sample and the Teflon housing to ensure that the inside of the Teflon housing is water-proof, and to avoid a short-circuit between the copper plate and the electrolyte. The sample is sandwiched between the copper electrode and the electrolyte, in which the whole Teflon housing is immersed. The

\footnotetext{
${ }^{3}$ Sulfur is an electron donor, and therefore GaP wafers doped with sulfur are n-type semiconductors.

${ }^{4}$ Suppliers are University Wafers, Atomergic Chemetals, Marketech and Ramet Ltd.

${ }^{5}$ The wafer doping concentrations are specified by the suppliers.

${ }^{6}$ Teflon and Viton are polymers and very resistant against chemicals.
} 
Figure 3.2: Cartoon of the energy diagram of the conduction $(C B)$ and valence $(V B)$ bands of a semiconductor. When the semiconductor is in contact with an electrolyte, the two bands are bent. When the bending is strong enough, electrons can tunnel between the $V B$ and $C B$, and therefore produce the holes necessary for etching at the interface with the acidic solution.

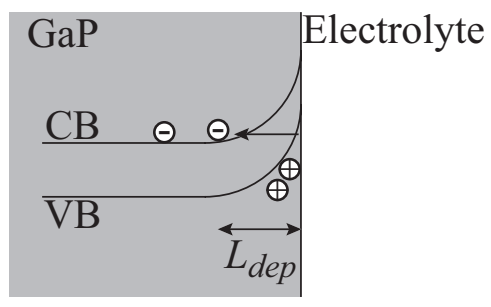

electrolyte is connected to a platinum sheet, of area $\sim 2 \mathrm{~cm}^{2}$, working as counter electrode. The potential between the two electrodes is set by a power supply, and the current flowing through the electrodes is monitored. The electrolyte is typically an $0.5 \mathrm{~mol} / \mathrm{L}$ aqueous sulfuric acid solution $\left(\mathrm{H}_{2} \mathrm{SO}_{4}\right)$, although different electrolytes can also be used to produce a porous structure. The temperature of the whole cell is kept constant by a thermostat, and usually at $21^{\circ} \mathrm{C}$. In section 3.2 .3 a study of the anodic etching vs. temperature is presented.

\subsubsection{Electrochemistry of GaP}

The dissolution of GaP into an acid follows the reaction equation [85, 87]

$$
(\mathrm{GaP})_{n}+6 \mathrm{~h}^{+} \rightarrow(\mathrm{GaP})_{n-1}+\mathrm{Ga}(\mathrm{III})+\mathrm{P}(\mathrm{III}) .
$$

The valence-band holes $\mathrm{h}^{+}$are produced in the case of the anodic etching by the tunneling of electrons from the valence to the conduction band. Indeed, as can be seen from Fig. 3.2. the valence and conduction bands of a semiconductor are bent at the interface with an electrolyte. When the electrolyte is at a lower potential than the semiconductor, the valence and conduction bands in the bulk of the semiconductor shift toward lower energy. When the potential difference between the semiconductor and the electrolyte is large enough 7 , electrons can tunnel from the valence to the conduction band, leaving holes behind. Under the influence of the electric field, the electrons move toward the bulk of the semiconductor, and the holes stay at the surface. Therefore, a relatively high concentration of holes is created at the surface of the semiconductor, where they are used to dissolve the semiconductor. The region in which the semiconductor bands are bent is the depletion layer $L_{\mathrm{dep}}$, and its width is [150]

$$
L_{\text {dep }}=\sqrt{\frac{2 \varepsilon \varepsilon_{0}}{e N}\left(V-V_{f b}\right)},
$$

where $\varepsilon \varepsilon_{0}$ is the electrical permittivity and $N$ the donor density of the semiconductor, $e$ the elementary charge, $V$ the applied potential, and $V_{f b}$ the potential at which the bands are flat. This flat-band potential depends on the semiconductor electrode and the electrolyte. For $\mathrm{GaP}$ in an $0.5 \mathrm{~mol} / \mathrm{L}$ aqueous sulfuric acid solution, $V_{f b} \simeq-1.2 \mathrm{~V}$ [86].

In order to characterize the behavior of a $\mathrm{GaP}$ electrode in an electrolyte, a currentdensity versus potential (or $\mathrm{i}-\mathrm{V}$ ) curve is measured. The potential between the two electrodes ( $\mathrm{GaP}$ on copper and platinum in electrolyte) is slowly scanned, at a rate of $50 \mathrm{mV} / \mathrm{s}$, between 0 and up to a maximum of $35 \mathrm{~V}$. The current is measured and normalized to the

\footnotetext{
${ }^{7}$ The potential difference, above which the tunneling of electrons is allowed, is called the breakdown potential.
} 


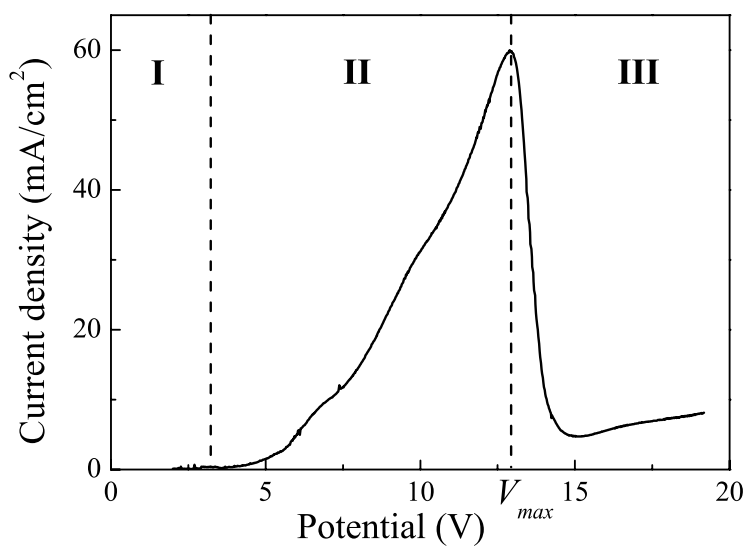

Figure 3.3: Typical current density-potential plot for a GaP electrode in $0.5 \mathrm{~mol} / \mathrm{L}$ aqueous sulfuric acid solution. The potential $V$ is scanned between $O V$ and $19 \mathrm{~V}$. The GaP wafer has $N=6 \times 10^{17} \mathrm{~cm}^{-3}$. In this system, a higher current density means a higher rate of etching.

surface area of the sample in contact with the electrolyte. Fig. 3.3 shows an example of such an i-V curve for a wafer of doping concentration $N=6 \times 10^{17} \mathrm{~cm}^{-3}$ in an $0.5 \mathrm{~mol} / \mathrm{L}$ aqueous sulfuric acid solution. A GaP electrode in sulfuric acid solution typically presents three potential ranges. In the region I of Fig. 3.3. at low potential, no etching occurs because the band bending is too small to allow the tunneling of electrons from valence to conduction band. Region II starts at the breakdown potential. Above the breakdown potential, electrons tunnel to the conduction band, and valence holes dissolve in the electrolyte. The anodic current increases with increasing potential. In region II, pores with size in the range 50-200 nm are produced. At even higher potential, above $V_{\max }$ (in region III), the current density drops to a low value before increasing slowly with potential. This decrease in current density is characteristic of the passivation of the GaP electrode, due to the formation of an oxide layer. A higher potential leads to a higher etching rate, but also to a higher rate of oxide formation. The potential of highest current density $V_{\max }$ marks the transition between the dissolution limited by the breakdown charge transfer and the dissolution limited by the oxide passivation. The potential $V_{\max }$ is found to be characteristic of the doping concentration of the $\mathrm{GaP}$ electrode and the electrolyte. In $0.5 \mathrm{~mol} / \mathrm{L}$ sulfuric acid solution, $V_{\max }$ decreases with increasing doping concentration, as can be seen in Fig. 3.4 .

\subsubsection{Formation of porous GaP}

At a constant potential, between the breakdown potential $(\sim 3 \mathrm{~V})$ and the potential of highest current density $V_{\max }$, (i.e., in region II of Fig. 3.3) a porous structure is formed. A scanning electron microscope (SEM) image of a typical example of a porous structure is shown in Fig. 3.5. In region II, the potential $V$ applied to the GaP electrode is not by itself enough to begin the dissolution. Etching starts at defects on the surface of the $\mathrm{GaP}$ electrode, where the electric field is enhanced [87]. The enhancement of the electric field makes possible the tunneling of the electrons to the conduction band and therefore 
Figure 3.4: Potential of maximum current density for GaP electrodes of different doping concentration in $0.5 \mathrm{~mol} / \mathrm{L}$ sulfuric acid solution.

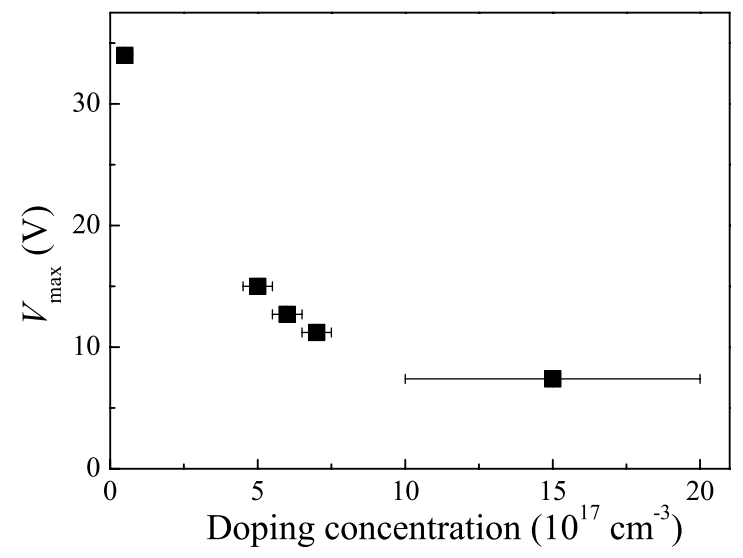

the dissolution of $\mathrm{GaP}$ in the electrolyte. As soon as pits in the surface are formed due to defects, the electric field is additionally enhanced by the curvature of the surface. The field enhancement is higher at the pore tips than at the pore walls. The pores therefore tend to grow longer, at their tip. When the field enhancement is too high, an oxide layer is formed that passivates the pore tips. Etching can now only occur close to the tips, where the field enhancement is just smaller. The etching does not always go on along the pore tips and therefore leads to branching of the pores [81]. The very irregular pattern of pores in Fig. 3.5 is due to this branching. To the eye, porous $\mathrm{GaP}$ is an opaque bright-yellow material. Bare $\mathrm{GaP}$ itself is transparent to visible light above $550 \mathrm{~nm}$, and looks orange-brown. The porous structure of $\mathrm{GaP}$ scatters light very strongly (see following chapters). The color of porous $\mathrm{GaP}$ is given by reflected light instead of the transmitted light through the bare $\mathrm{GaP}$. In reflection, light can travel a very short path in the material before escaping, and the absorption in these short paths is minimal. Reflected light has therefore more frequency components from the green part of the spectrum, below $550 \mathrm{~nm}$, than transmitted light. With increasing scattering strength, the porous GaP samples look more yellow.

Anodic etching of $\mathrm{GaP}$ in $0.5 \mathrm{~mol} / \mathrm{L}$ sulfuric acid solution allows the formation of a homogeneous layer of porous material. The thickness of the porous layer is determined by the etching time, or equivalently the total etching charge [151], and can be made at will between a few microns and the thickness of the wafer $(\sim 300 \mu \mathrm{m})$. Because the pores are initiated at pits on the surface, the surface itself is scarcely porous. This 'non-porous'

Figure 3.5: SEM image of a longitudinal cross section of a porous structure. The GaP wafer with $N=5 \times 10^{17} \mathrm{~cm}^{-3}$ was etched at $14 \mathrm{~V}$ in $0.5 \mathrm{~mol} / \mathrm{L}$ sulfuric acid solution. The pores (darker to black regions in the picture) grow from the bottom of the picture. Scale bar $=1 \mu \mathrm{m}$.

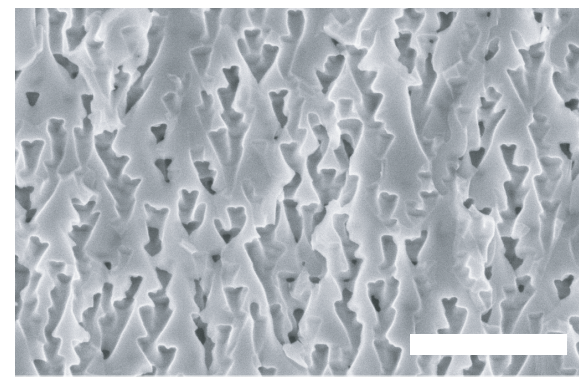



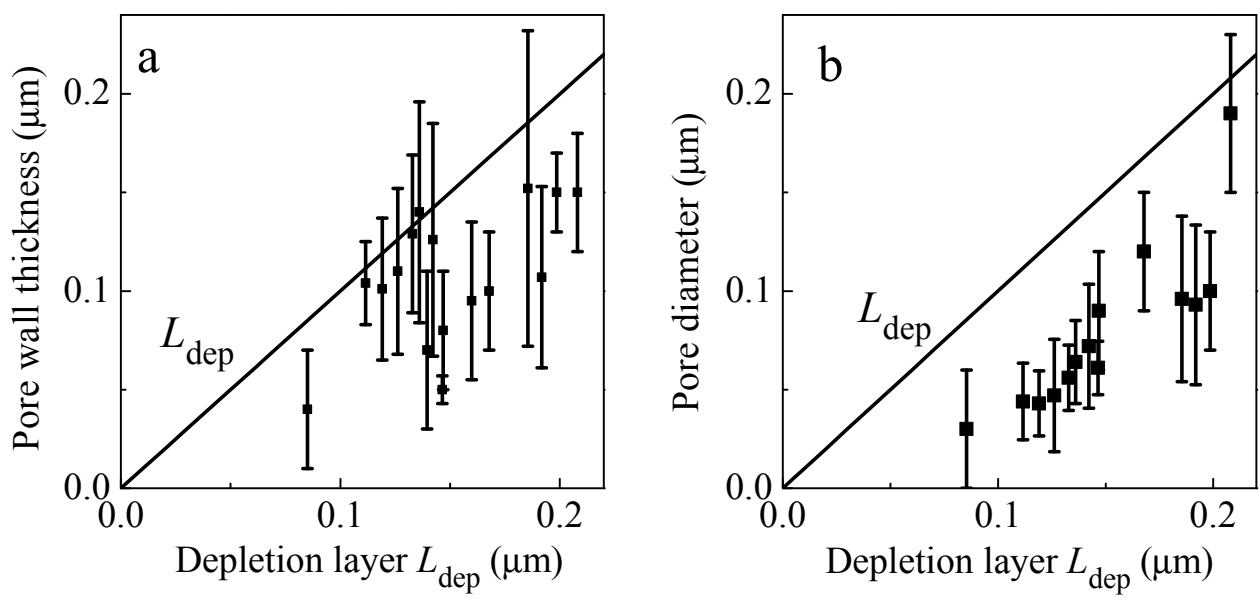

Figure 3.6: Average pore wall thickness (a) and pore diameter $(b)$ of a range of porous samples (different doping concentration and etched at different potentials, in $0.5 \mathrm{~mol} / \mathrm{L}$ sulfuric acid) compared to the width of the depletion layer $L_{\mathrm{dep}}$.

top-layer has a thickness $\sim 200 \mathrm{~nm}$. The naked eye can easily detect the strong specular reflection from the top-layer. Such a top-layer has to be removed in order to simplify the analysis of the optical measurements (see section 4.3.2). The removal of the top-layer is described further in section 3.3.1

The typical size of the pores is of particular interest to the studies of light scattering. The tangential growth of a pore is limited by the vicinity of other pores. Two pores next to each other will grow in diameter up to the point where their depletion layers overlap. In the $\mathrm{GaP}$ wall between two pores which are less than twice the width of the depletion layer apart, the electric field is no longer enhanced, so that the tunneling of the electrons, and therefore the etching, stops [144, 152]. In Fig. 3.6 the thickness of the pore walls and the pore diameters in porous samples are compared to the depletion layer width (see Eq. 3.2). The range of porous samples spans different doping concentrations $\left(5-15 \times 10^{17} \mathrm{~cm}^{-3}\right)$ and voltages (up to $V_{\max }$ ). Both characteristic lengths of the porous structure (pore diameter and wall thickness) are close to the width of the depletion layer $L_{\mathrm{dep}}$ in the range of high doping concentration (above $5 \times 10^{17} \mathrm{~cm}^{-3}$ ) and low potential (the maximum $V_{\max }$ for these doping concentrations is $15.1 \mathrm{~V})$. The wafer of lowest doping concentration available $\left(N=0.5 \times 10^{17} \mathrm{~cm}^{-3}\right)$, etched in sulfuric acid solution at $32 \mathrm{~V}$, has a much wider depletion layer $\left(L_{\mathrm{dep}}=0.89 \mu \mathrm{m}\right)$ but its porous structure does not have a much bigger characteristic length $($ wall thickness $=(186 \pm 55) \mu \mathrm{m}$ and pore diameter $=(194 \pm 58) \mu \mathrm{m})$. Unfortunately the depletion layer width cannot easily be tuned between $0.2 \mu \mathrm{m}$ and $0.9 \mu \mathrm{m}$. Indeed, hardly any $\mathrm{GaP}$ wafers of intermediate doping concentration $\left(1-4 \times 10^{17} \mathrm{~cm}^{-3}\right)$ are available. Pores in the $\mathrm{GaP}$ wafer with low doping concentration could not be obtained at all below $30 \mathrm{~V}$. The largest pore size reliably attainable by anodic etching of $\mathrm{GaP}$ in sulfuric acid is $200 \mathrm{~nm}$. Section 3.3.2 describes a method to further increase the diameter of the pores by an additional etching step.

It can be argued that the limitation in the maximum pore size obtained by anodic etch- 
ing of $\mathrm{GaP}$ is due to the choice of electrolyte. Apart from $0.5 \mathrm{~mol} / \mathrm{L}$ sulfuric acid solution (which was used to produce all the samples presented in this thesis) both concentration and nature of the acid in the electrolyte can be changed. Two other acids, namely nitric acid $(0.1$ and $\left.1 \mathrm{~mol} / \mathrm{L} \mathrm{HNO}_{3}\right)$ and phosphoric acid $\left(1 \mathrm{~mol} / \mathrm{L} \mathrm{H}_{3} \mathrm{PO}_{4}\right)$ and a lower concentration of sulfuric acid $(0.05 \mathrm{~mol} / \mathrm{L})$ were tested as electrolyte for anodic etching of GaP. Comparable results are obtained using these different acids and concentrations [153]. The main difference between etching at different concentrations is the rate of dissolution of GaP. Within the same potential range in the three different acids, comparable porous structures, including pore sizes, are obtained. Nevertheless, in nitric acid, the oxide-formation regime (region III in Fig. 3.3) does not occur. The porous structure formed in nitric acid at potentials higher than the $V_{\text {max }}$ found in sulfuric acid is not mechanically stable: the structure crumbles as a powder when the sample is extracted from the etching setup. The samples etched in nitric acid at high potential are therefore unusable.

Apart from the size of the pores, an important parameter of the porous structure is its anisotropy. Of course, the pores in $\mathrm{GaP}$ are grown from the interface with the electrolyte in a direction normal to the surface of the wafer. As can be seen from Fig. 3.5, the porous structure has an obvious preferential direction. Chapter 6 describes the spatial anisotropy in multiple light scattering from such anisotropic porous structures. It has been qualitatively observed that etching at higher potential leads to a more isotropic structure.

\subsubsection{Temperature dependence}

Temperature is a parameter which influences the rates of chemical reactions. The law of Arrhenius states that the rate of a chemical reaction scales with $\exp \left(-\mathrm{E}_{\mathrm{a}} / \mathrm{k}_{\mathrm{B}} \mathrm{T}\right)$, where $\mathrm{E}_{\mathrm{a}}$ is the activation energy of the reaction, $\mathrm{k}_{\mathrm{B}}$ the Boltzmann constant, and $\mathrm{T}$ the temperature. According to studies on the anodic etching of Si $[145,146]$, the temperature indeed increases the rate of etching. It was suggested [81] that increasing the temperature of the electrolyte would accelerate the dissolution of the passivating oxide layer in the etching of $\mathrm{GaP}$. The potential $V_{\max }$ at which the etching dynamics changes from charge-transfer limited to oxide-formation limited should therefore increase. According to the width of the depletion layer $L_{\mathrm{dep}}$ (see Eq. 3.2) and the pore size dependence in the range $L_{\mathrm{dep}}=(0.05-$ $0.25) \mu \mathrm{m}$, etching at a higher potential (up to $V_{\max }$ ) should increase the size of the pores.

A GaP wafer of doping concentration $N=7 \times 10^{17} \mathrm{~cm}^{-3}$ was etched in $0.5 \mathrm{~mol} / \mathrm{L}$ sulfuric acid solution, at a constant temperature in the range $20-60^{\circ} \mathrm{C}$. The potential $V_{\max }$ of highest current density was found to be independent of temperature, within the experimental uncertainty $(\sim 0.2 \mathrm{~V})$. The current density, at a given potential, increased exponentially with temperature. Fig. 3.7 shows the temperature dependence of the current density at 11.2 $\mathrm{V}=V_{\max }$. From the fit to Arrhenius law in Fig. 3.7, the activation energy of the etching reaction is found as $\mathrm{E}_{\mathrm{a}}=(0.24 \pm 0.03) \mathrm{eV}$, comparable to the value found for $\mathrm{Si}$ [145]. The etching mechanics are therefore expected to be similar for $\mathrm{Si}$ and $\mathrm{GaP}$.

The distribution of pore size in the GaP samples was found to be independent of the etching temperature, within the experimental accuracy $(\simeq 5 \%)$. Etching GaP at a higher temperature leads to a faster rate of the reaction, but no change in the porous structure. 


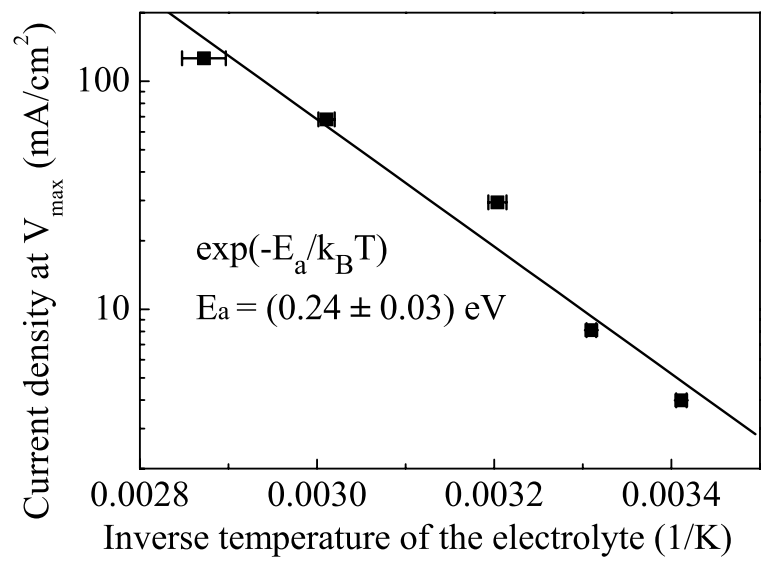

Figure 3.7: Current density (in logarithmic scale) versus the (inverse) temperature of the electrolyte. The solid line is a fit to Arrhenius law, giving the activation energy $E_{\mathrm{a}}$ of the chemical reaction.

\subsubsection{Electroluminescence}

Although a typical anodic etching experiment is done in the dark, we observed that the porous $\mathrm{GaP}$ layer produces light during etching. Further experiments [91] showed that electrons excited above the breakdown potential give rise to a broadband 'electroluminescence' at the pore tips. This luminescence covers a broad spectrum, $450 \mathrm{~nm}$ to at least $1 \mu \mathrm{m}$. As the thickness of the sample increases during etching, light produced at the pore front has to cross the multiple scattering layer before escaping. It is possible to argue that monitoring the luminescence spectrum as a function of time (i.e., thickness) allows an in situ measure of the total transmission through the sample [154]. At a wavelength below the band gap of $\mathrm{GaP}$, absorption dominates and an exponential decay with the thickness of the porous layer is found. Above the band gap, absorption is negligible, and the total transmission follows the theoretical expectation ${ }^{8}$ in the diffusion regime (see Eq. 2.62). The transport mean free path of the diffusive porous material can be determined, provided the extrapolation ratio (i.e., the interface property, see chapter 4) of the sample is known, or assumed. Of course, the porous sample is infiltrated with water during etching, which reduces the index contrast of the scatterers, and therefore greatly increases the transport mean free path of the filled sample. Measurements on the dry and wet samples are not the same. Nonetheless, such a measurement in a filled sample is important to ensure that a less strongly scattering sample fulfills the diffusion approximation [83].

\subsection{Further chemical processing of porous GaP}

\subsubsection{Photochemical etching}

The electrochemical etching of $\mathrm{GaP}$ produces a porous layer. In the thin non-porous toplayer $(\sim 200 \mathrm{~nm})$, a small density of pits is present, indicating the points at which the pores

\footnotetext{
${ }^{8}$ It can be argued that the case of a light source exactly on the interface of the diffusive medium, as the luminescence from the pore tips can be described, does not exactly follow the geometry for the total transmission presented in the theory chapter. The applicability of the total transmission expectation (Eq. 2.62] is not discussed in reference [154].
} 
start to form. A SEM picture of the top of a typical sample can be seen in Fig. 3.8. The density of pits depends on the polishing of the surface, the doping concentration of the wafer and the anodic etching potential. The presence of the top-layer is obvious to the naked eye because of the strong specular reflection it produces. Sections 4.3.2 and 5.2.1 both show how much the top-layer influences the diffusion measurements performed on the porous samples.

In order to remove this top-layer either pre- or post-processing of the anodically-etched sample has to be done. Before anodic etching, an increase of the density of surface defects [155] leads to an increase in the density of pits and therefore increases the porosity of the top-layer, effectively merging it with the bulk porous structure. After anodic etching, further chemical polishing, using an aqueous bromine $\left(\mathrm{Br}_{2}\right)$ solution, removes the top-layer altogether [85]. Chemical polishing with the $\mathrm{Br}_{2}$ solution is very fast, and the removal of the top-layer cannot be easily controlled in this way.

Control over the removal of the top-layer is obtained by using photochemical etching [81]. Apart from the potential in anodic etching, electron-hole pairs can be produced by absorption of light in the semiconductor. These valence holes are subsequently used to oxidize the GaP, which dissolves in the acid, as in section 3.2. The beam from an argon-ion laser of wavelength $460 \mathrm{~nm}$, and power $20 \mathrm{~mW}$, hits the surface of the porous sample, at an incidence angle $\sim 45^{\circ}$. The porous sample is immersed in a solution consisting of 5 parts of $0.5 \mathrm{~mol} / \mathrm{L}$ aqueous sulfuric acid solution and 1 part of $30 \%$ aqueous hydrogen peroxide $\left(\mathrm{H}_{2} \mathrm{O}_{2}\right)$. The wavelength of the laser being smaller than the absorption edge of $\mathrm{GaP}$, the incident light is absorbed by the sample on short distances. Although the absorption length of GaP at $460 \mathrm{~nm}$ is about $3.5 \mu \mathrm{m}$ [137], most of the incident light is absorbed in the toplayer, since the underlying layer is diffusive. Most of the electron-hole pairs are therefore created within the top-layer. The free electrons reduce $\mathrm{H}_{2} \mathrm{O}_{2}$ to produce an ion $\mathrm{OH}^{-}$and a radical $\mathrm{OH}^{\bullet}$ [156]. The radical can further be reduced by hole-injection into the valence band, so that the original electron reacts with $\mathrm{H}_{2} \mathrm{O}_{2}$ and produces a hole in the valence band:

$$
\mathrm{H}_{2} \mathrm{O}_{2}+e^{-} \rightarrow 2 \mathrm{OH}^{-}+\mathrm{h}^{+} .
$$

The photogenerated and injected holes both contribute to the dissolution of $\mathrm{GaP}$ in the sulfuric acid solution (see Eq. 3.1). The GaP is therefore selectively etched where the incident beam is absorbed, namely in the top-layer.

The specular reflection of the incident beam on the porous GaP sample is monitored during the photochemical etching. The specular reflection during photochemical etching of a porous GaP sample (of doping concentration $N=7 \times 10^{17} \mathrm{~cm}^{-3}$ and anodically etched at a potential $V=11 \mathrm{~V}$ ) is shown in Fig. 3.8 The initial intense specular reflection gradually decays to a low value, after typically 20 minutes of photochemical etching. The laser beam is then blocked and the sample removed from the solution to stop the etching. The low value of the reflection at the end of etching is due to the small contribution from the diffuse reflection. The specular reflection of samples from well-polished $\mathrm{GaP}$ wafer displays interference fringes at the beginning of the photochemical etching. The reflection from the front and back surface of the top-layer gives rise to alternating constructive and destructive interferences as the thickness of the top-layer is reduced.

In Fig. 3.8 two SEM pictures of the surface of the porous sample before and after photochemical etching are also displayed. Before removal of the top-layer, in Fig. 3.8. 


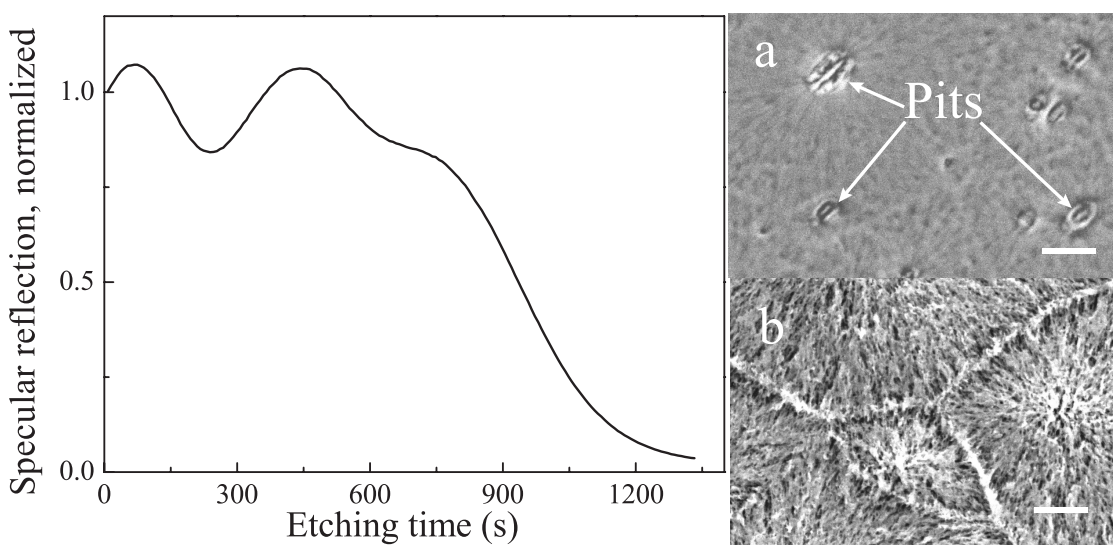

Figure 3.8: Photochemical etching of porous GaP. On the left, the specular reflection of the surface of a typical sample vanishes with etching time. The reflection is normalized to the initial value. On the right, SEM pictures of a porous GaP sample before (a) and after (b) photochemical etching, where the top-layer is etched away. Scale bars $=2 \mu \mathrm{m}$.

the surface of the sample is mainly flat, with occasional pits. After removal of the toplayer, in Fig. 3.8p, the porous structure is uncovered. The thicker white lines in Fig. $3.8 \mathrm{p}$, spreading radially from the center of the image, are the walls between adjacent porous domains. Enclosed by one such thick line is a porous structure originating from one single pit, i.e., in fact, a single pore with numerous branches.

\subsubsection{Chemical etching}

As is seen in section 3.2.2, the pore size can easily be tuned from $50 \mathrm{~nm}$ to $200 \mathrm{~nm}$ via the proper choice of $\mathrm{GaP}$ wafer and anodic etching conditions. One way to increase the available range of pore size is to chemically etch the porous samples. As noted in the previous section on photochemical etching, free conduction electrons can reduce the $\mathrm{H}_{2} \mathrm{O}_{2}$ and produce valence holes (see Eq. 3.3. These valence holes oxidize the GaP which slowly dissolves in the sulfuric acid solution. Immersing a porous $\mathrm{GaP}$ sample inside the same solution as for photochemical etching leads to a slow chemical etching of the porous structure [81], even without illumination from the argon-ion laser. The average diameter of the pores increases uniformly in the whole sample. Note that chemical etching is much slower than photochemical etching. Therefore, in the (relatively) short time needed to photochemically etch a porous sample, the sample is hardly affected by chemical etching.

\section{Increasing the pore size}

In order to increase the diameter of the pores, a porous sample, after anodic etching to produce the porous layer and photochemical etching to remove the top-layer, is immersed in a solution consisting of 5 parts $0.5 \mathrm{~mol} / \mathrm{L} \mathrm{H}_{2} \mathrm{SO}_{4}$ and 1 part $30 \% \mathrm{H}_{2} \mathrm{O}_{2}$. A Teflon tape with an opening protects the non-etched part of the sample. In order to monitor the chemical etching, the total transmission of a helium neon $(\mathrm{HeNe}$ ) laser beam (at $633 \mathrm{~nm}$ ) through the 


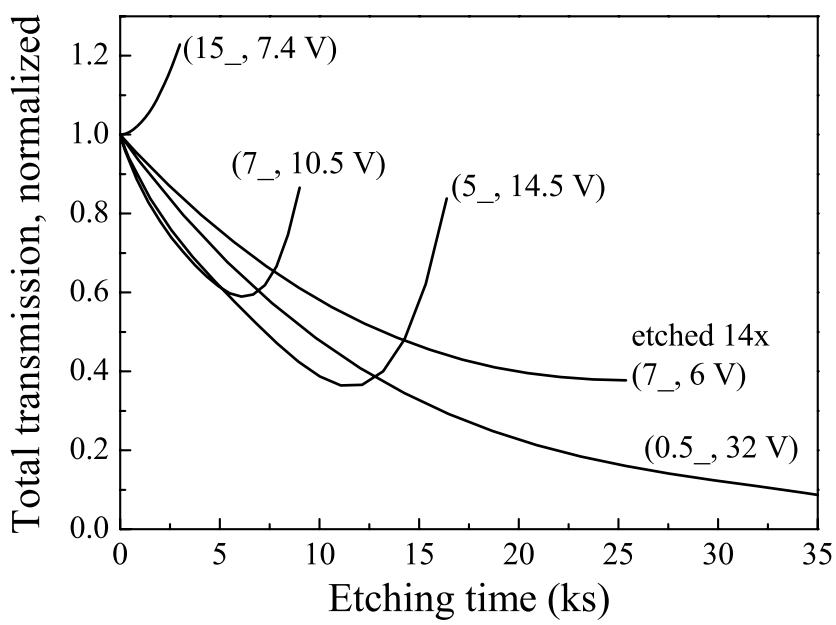

Figure 3.9: In situ total transmission of a HeNe laser beam (633 nm) through porous GaP during chemical etching. Each curve corresponds to a different sample, labeled with the doping concentration of the wafer (where $1_{-} \equiv 10^{17} \mathrm{~cm}^{-3}$ ) and the anodic etching potential. The $4^{\text {th }}$ curve was chemically etched 14 times for $1.8 \mathrm{ks}$ each time. Chemical etching increases the size of the pores. A lower value of the total transmission typically means a more strongly scattering sample.

sample is measured as a function of etching time. In order to avoid two-photon absorption, the incident beam is attenuated. Without attenuation, the absorption of the laser beam in the sample leads to the faster, but depth-dependent, photochemical etching, which must be avoided.

In Fig. 3.9 the total transmission through different porous samples is plotted as a function of chemical-etching time, and normalized to the initial transmission. Each curve corresponds to a porous sample of different doping concentration, or etched at a different etching potential. The curves are labeled as: (doping concentration in $10^{17} \mathrm{~cm}^{-3}$, etching potential). From the sample chemically-etched for the longest time, the rate of chemical etching is estimated to be $\sim 6 \mathrm{~nm} / \mathrm{ks}$.

The total transmission of the HeNe laser beam through a sample of thickness $L$ filled with water scales as $\ell\left(1+\tau_{\mathrm{e}}\right) / L$, where $\ell$ is the transport mean free path of the porous structure filled with water, and $\tau_{\mathrm{e}}$ is the extrapolation ratio (see sections 2.5.1 and 4.2): a property of the interface between water and the filled porous structure. A decrease of total transmission is an indication that the transport mean free path of the filled porous structure also decreases. Note that the mean free path has no bounds, whereas the extrapolation ratio is typically in the range 1-3 and varies slowly with the effective refractive index of the porous material, i.e., with the porosity of the sample (see section 4.4). The extrapolation ratio decreases slowly with increasing porosity of the sample, and therefore decreases with chemical-etching time. For typical samples, of thickness much bigger than the transport mean free path, the change in thickness during chemical etching is negligible.

In Fig. 3.9, all but the leftmost curve decrease, go through a minimum, and increase again if the chemical etching is continued. The leftmost curve, for the sample of highest doping concentration and smallest pore wall thickness (see section 3.2.2), does not go 
through a minimum but increases from the start. Since the diameter of the pores in the GaP samples presented here is typically in the range 50 to $200 \mathrm{~nm}$, their scattering strength is expected to increase when the pore size is closer to the wavelength in the material, i.e., bigger. Pores are etched, and their diameter increases slowly. At the beginning of the etching, the scattering strength therefore increases, and the total transmission decreases. Of course, if the chemical etching goes on for too long, the porous structure completely dissolves, and therefore its scattering strength decreases, the total transmission increases. There is an optimal diameter of the pores, at a given pore density, where the scattering strength is the highest. This optimal diameter corresponds to the minimum in total transmission during chemical etching. In order to get the strongest scattering chemically-etched sample from a given initial sample, the etching is stopped at the minimum in transmission. The leftmost curve in Fig. 3.9 does not have a minimum since the optimal diameter of the pores for the pore density corresponding to this sample is smaller than the pore diameter at the beginning of the chemical etching.

\section{Homogeneity of the chemical etching}

The chemical etching allows the slow increase of the pore diameter of porous GaP samples. This etching method has been used [81] to increase the scattering strength of the GaP samples. Unfortunately, a discrepancy between two optical measurements on chemicallyetched samples was found in reference [81]. Although the in situ total transmission of a particular sample was found to decrease with chemical etching, the mean free path measured from EBS was seen to increase. Total transmission measurements are sensitive to the scattering properties in the whole bulk of the sample. EBS is a measurement of reflected light, which mainly probes a small layer (about a few mean free paths thick) of scattering material, on top of the sample. It is very important to monitor the homogeneity of the chemically-etched sample.

In the previous study [81], the chemical etching was set up as follows: the porous sample was immersed in the $\mathrm{H}_{2} \mathrm{SO}_{4}$ solution, and the extra $\mathrm{H}_{2} \mathrm{O}_{2}$ was injected afterwards in the acid solution. In that scheme, the $\mathrm{H}_{2} \mathrm{O}_{2}$ has to diffuse from the acid solution into the pores of the sample. Such a diffusion process of chemical compounds is not instantaneous, especially if no agitation is applied to the solution. The diffusion through the narrow pores of the sample is an extra hindrance for the homogenization of the $\mathrm{H}_{2} \mathrm{O}_{2}$ concentration.

As a test of the uniformity of the chemical-etching rate, a sample, of doping concentration $N=7 \times 10^{17} \mathrm{~cm}^{-3}$ and anodically etched at a potential of $6 \mathrm{~V}$, is chemically etched 14 times for $30 \mathrm{~min}$ each time. The total transmission of these 14 subsequent etchings is plotted, one after the other, in Fig. 3.9. A picture from an optical microscope of a cleaved edge of the sample chemically etched 14 times is shown in Fig. 3.10. The porous layer of this sample is $\sim 200 \mu \mathrm{m}$ thick. The porous layer is obviously not homogeneous after chemical etching. Two regions, of ill-defined interface, of barely etched pores (small pores) and chemically-etched pores (bigger pores), are observed. The surface of the sample is not flat due to the additional chemical etching close to the surface. In 30 min, the $\mathrm{H}_{2} \mathrm{O}_{2}$ has only diffused about $100 \mu \mathrm{m}$ inside the porous structure. Relying on the diffusion of the molecules in the solution to homogenize the acid concentration is therefore ill-advised.

In order to circumvent the problem of the homogeneity of the etching solution, the 
Figure 3.10: Optical image of the cross section of a sample chemically etched 14 times for $30 \mathrm{~min}$. The whole thickness ( $300 \mu \mathrm{m})$ of the sample is visible. The porous region (top $200 \mu \mathrm{m}$ ) is obviously inhomogeneous due to a wrong modus operandi of the chemical etching. Scale bar $=100 \mu \mathrm{m}$.

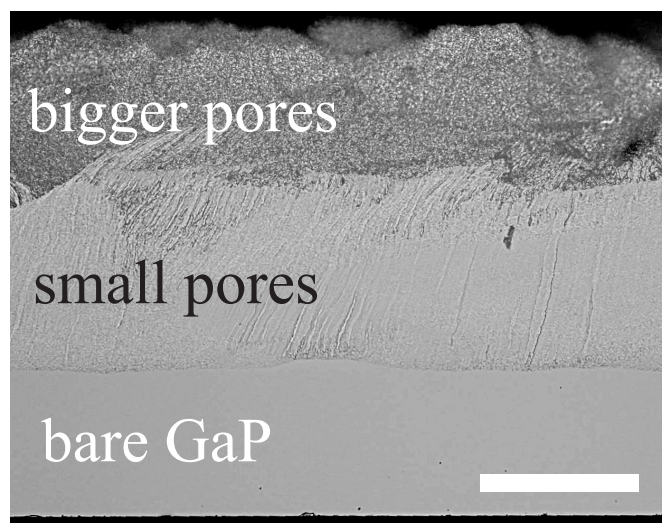

mixing of the two components, $\mathrm{H}_{2} \mathrm{SO}_{4}$ and $\mathrm{H}_{2} \mathrm{O}_{2}$, has to be done before immersion of the sample. The sample is only then immersed in the etching solution. Bubbles from air still trapped inside the pores slowly rise from the surface of the porous sample. Lightly shaking the sample provokes a flurry of bubbles to rise from the surface, after which the sample is fully infiltrated with the etching solution. Chemical etching then proceeds at a uniform rate across the whole sample. Porous samples chemically etched following this second immersion procedure were found to be homogeneous.

\subsection{Conclusions}

Electrochemical etching of $\mathrm{GaP}$ allows the formation of a homogeneous porous structure of well-defined thickness. Using different doping concentrations of the $\mathrm{GaP}$ wafer or etching potentials, one can tune the typical size of the pores in the range 50-200 nm. After electrochemical etching, a top-layer of bare GaP remains, which can be removed in a controlled way by photochemical etching. After photochemical etching, the porous sample has well-defined optical properties. The size of the pores of a porous sample can be increased by chemical etching. Following the right experimental scheme, the porous sample remains homogeneous after chemical etching. 



\section{Chapter 4}

\section{Diffusion at the interface}

\subsection{Introduction}

Strictly speaking, diffusion does not extend up to the interface of a diffusive medium. Socalled skin layers [157], where light crosses the transition from ballistic to diffusive, are not described by diffusion. Nonetheless, the careful treatment of the interface in the diffusion regime allows analytical solutions to be found which are tested in special cases against the more rigorous theory.

In section 2.4 the diffusion equation for the infinite medium (Eq. 2.53) is derived. In section 2.5 the diffusion equation is applied to a slab, in order to obtain predictions for a real experiment. Applying the diffusion equation to a finite medium requires the use of boundary conditions, which are derived in the first section of this chapter, following Ref. [128]. A closely related subject to the boundary condition at an interface is the determination of the angular dependence of light escaping the diffusive medium. Such an 'escape function' is described in section 4.2.2. and shown to depend only on the effective refractive index of the diffusive medium. In section 4.3 , the theoretical predictions are tested in the case of strongly scattering slabs and shown to describe porous GaP samples very well. The third section presents a study of the effective refractive index in a range of strongly scattering samples with different geometrical and scattering properties. Effective medium theories fail to account for the behavior of the refractive index versus porosity in strongly scattering porous $\mathrm{GaP}$ samples.

\subsection{Theory of the interface of a diffusive medium}

In principle, the solution for the Green function of a finite system should be calculated by considering the exact distribution of scatterers in the whole space. Most of space contains no scatterers, i.e., vacuum. The diffusive medium is a region of space with a certain density of scatterers. In such a description, the Green function gives the exact result for a finite system, but is very difficult to solve. Indeed, in section 2.4, the statistical invariance by any translation was a major help in order to derive the Boltzmann equation (Eq. 2.39) and the diffusion equation (Eq. 2.53). An exact result for a semi-infinite slab containing 


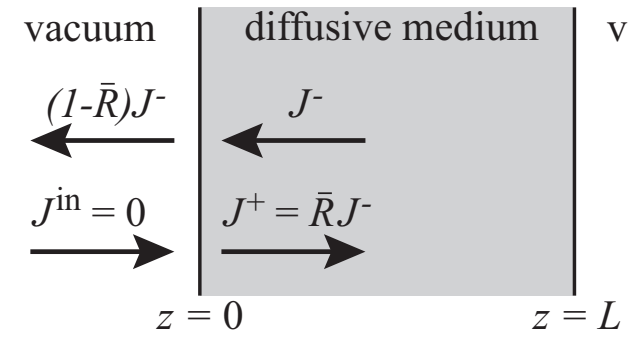

Figure 4.1: Cartoon of the slab geometry, a diffusive medium sandwiched by vacuum, with interfaces at $z=0$ and $z=L$. The diffuse flux $J^{\text {in }}=0$ corresponds to the strict boundary condition between free space and a diffusive material. $J^{+}=\bar{R} J^{-}$is the boundary condition with averaged internal reflectivity $\bar{R}$. The fluxes are represented at the left interface, but should be read as symmetrical at the right interface.

point scatterers can be found within the radiative transfer equation, and is known as the Milne problem [158]. It is more practical to use a diffusion Green function for the infinite medium, apply boundary conditions to mimic the finite system at the interfaces, and only consider the resulting solution within the finite medium.

The strict boundary condition for an interface between a medium with multiple scattering and free space is that no diffuse light can enter the material through the interface. The specific intensity $P\left(\frac{\omega}{c} \hat{\mathbf{p}}, \mathbf{r}\right)$ (see Eq. 2.47) is the diffuse intensity at position $\mathbf{r}$ and in the direction $\hat{\mathbf{p}}$. The strict boundary condition can be written as

$$
P\left(\mathbf{p}_{\text {in }}, \mathbf{r}_{\text {int }}\right)=0 \text { where }\left\{\begin{array}{l}
\mathbf{r}_{\text {int }} \text { is on the interface and } \\
\mathbf{p}_{\text {in }} \text { pointing inside the material. }
\end{array}\right.
$$

\subsubsection{Boundary conditions to the diffusion equation}

Within the diffusion approximation, the distribution of diffuse light is almost isotropic (see the development of $P(\mathbf{p}, \mathbf{r})$ in first order of $\mathbf{p}$, in Eq. 2.47). It follows that the strict boundary condition is impossible to satisfy within the diffusion approximation. An approximate boundary condition can be considered, where the total flux $J^{\text {in }}$ entering through the interface has to vanish.

$$
J^{\text {in }} \equiv \int_{2 \pi} P\left(\mathbf{p}_{\text {in }}, \mathbf{r}_{\text {int }}\right)\left(\mathbf{p}_{\text {in }} \cdot \hat{\mathbf{s}}\right) \mathrm{d} \mathbf{p}_{\text {in }}=0 \text { where }\left\{\begin{array}{l}
\mathbf{r}_{\text {int }} \text { is on the interface and } \\
\mathbf{p}_{\text {in }} \text { pointing inside the material. }
\end{array}\right.
$$

The vector $\hat{\mathbf{s}}$ is normal to the interface, pointing inside the material, and $\mathbf{p}_{\text {in }}$ is integrated over a half space, inward.

One refinement to this approximate boundary condition is that the total diffuse flux $J^{+}$going inward from the interface is the reflection of the total diffusive flux $J^{-}$going outward to the interface. By denoting $\bar{R}$ the average reflectivity coefficient at the interface the reflective boundary condition reads $J^{+}=\bar{R} J^{-}$(See Fig. 4.1).

The diffuse fluxes can easily be evaluated in the case of isotropi 11 and weakly-absorbing scatterers $\left(\ell \ll \ell_{\mathrm{a}}\right)$. The flux scattered directly from a volume $\mathrm{d} V$ inside the medium to a surface $\mathrm{d} S$ on the interface is given by

$$
\mathrm{d} J \mathrm{~d} S=W_{\omega}^{\mathrm{rad}}(r, \theta, \phi) \mathrm{d} V \frac{\nu_{\mathrm{E}}}{\ell} \frac{\cos \theta}{4 \pi r^{2}} \exp \left(-\frac{r}{\ell}\right) \mathrm{d} S,
$$

\footnotetext{
${ }^{1}$ The generalization to anisotropic scattering leads to renormalize the scattering mean free path as the transport mean free path $[9,159]$.
} 
where the spherical coordinates $(r, \theta, \phi)$ are chosen, with $\theta$ the declination angle from the $z$-axis and $\phi$ the azimuthal angle. The energy density in the volume $\mathrm{d} V, W_{\omega}^{\mathrm{rad}}(r, \theta, \phi) \mathrm{d} V$ is scattered isotropically and a fraction $\left(\cos \theta / 4 \pi r^{2}\right) \mathrm{d} S$ flows toward the surface $\mathrm{d} S$. Only a fraction $\exp (-r / \ell)$ will not be scattered between $\mathrm{d} V$ and $\mathrm{d} S$. The rate at which diffusion occurs is the inverse of the mean free time, or $v_{\mathrm{E}} / \ell$.

Integrating Eq. 4.3 over the half space $z>0$ gives the outward flux $J^{-}$:

$$
J^{-}=\frac{v_{\mathrm{E}}}{4 \pi \ell} \int_{0}^{\infty} \int_{0}^{2 \pi} \int_{0}^{\pi / 2} W_{\omega}^{\mathrm{rad}}(r, \theta, \phi) \exp \left(-\frac{r}{\ell}\right) \cos \theta \sin \theta \mathrm{d} \theta \mathrm{d} \phi \mathrm{d} r
$$

which leads to

$$
J^{-}=\frac{\nu_{\mathrm{E}}}{4} W_{\omega}^{\mathrm{rad}}(z=0)+\left.\frac{\nu_{\mathrm{E}} \ell}{6} \frac{\partial W_{\omega}^{\mathrm{rad}}(z)}{\partial z}\right|_{z=0},
$$

where the dominant contribution to the integral of Eq. 4.4 comes from the first transport mean free path, due to the exponential term. The diffusion approximation further allows the use of the first-order Taylor expansion of the energy density along the $z$-axis in Eq. 4.5 Performing the integration over the $z<0$ half-space gives for the inward flux

$$
J^{+}=\frac{\nu_{\mathrm{E}}}{4} W_{\omega}^{\mathrm{rad}}(z=0)-\left.\frac{\nu_{\mathrm{E}} \ell}{6} \frac{\partial W_{\omega}^{\mathrm{rad}}(z)}{\partial z}\right|_{z=0} .
$$

The reflective boundary condition, linking the two fluxes, inward and outward, to the average reflectivity at the interface, now reads

$$
\begin{array}{r}
W_{\omega}^{\mathrm{rad}}(z)-z_{\mathrm{e}_{1}} \frac{\partial W_{\omega}^{\mathrm{rad}}(z)}{\partial z}=0 \text { at } z=0, \\
\text { with } z_{\mathrm{e}_{i}} \equiv \frac{2}{3} \frac{1+\bar{R}_{i}}{1-\bar{R}_{i}} \ell \equiv \tau_{\mathrm{e}_{i}} \ell .
\end{array}
$$

Performing the same steps for a half-space diffusive for $z<L$ gives the second boundary condition needed in the case of a slab:

$$
W_{\omega}^{\mathrm{rad}}(z)+z_{\mathrm{e}_{2}} \frac{\partial W_{\omega}^{\mathrm{rad}}(z)}{\partial z}=0 \text { at } z=L
$$

The quantity $z_{\mathrm{e}}$ is called the extrapolation length (and $\tau_{\mathrm{e}}$ the extrapolation ratio) since the mixed boundary conditions Eqs. 4.7 and 4.9 describe a vanishing extrapolation of the diffuse intensity at a distance $z_{\mathrm{e}}$ outside the interface. Eqs. 4.7 and 4.9 can equivalently be written, in the elastic or weakly absorbing regime, as

$$
W_{\omega}^{\mathrm{rad}}(z)=0 \text { at }\left\{\begin{array}{l}
z=-z_{\mathrm{e}_{1}} \\
z=L+z_{\mathrm{e}_{2}} .
\end{array}\right.
$$

Eq. 4.8 reduces to $\tau_{\mathrm{e}}=2 / 3$ in the case of a fully transparent interface $(\bar{R}=0)$. The solution to the Milne problem, considering the radiative transport equation and the strict boundary condition (Eq. 4.1), has the same qualitative behavior as here derived (Eq. 4.7) but with a slightly different quantitative value $\tau_{\mathrm{e}} \approx 0.7104$ [104, 118]. Further approximation on 
the Milne problem allows the inclusion of a finite reflectivity at the interface and leads to results equivalent to Eq. 4.8[157].

The average reflectivity coefficient $\bar{R}$ can be quantitatively found by considering the Fresnel reflection coefficients at the interface. A flat interface between two media of welldefined refractive index is considered. Using an angular-dependent reflectivity instead of $J^{+}=\bar{R} J^{-}$leads to

$$
\begin{gathered}
J^{+}=\int_{0}^{\pi / 2} R(\theta) J^{-}(\theta) \mathrm{d} \theta \\
=\frac{v_{\mathrm{E}}}{2} W_{\omega}^{\mathrm{rad}}(z=0) C_{1}+\left.\frac{v_{\mathrm{E}} \ell}{2} \frac{\partial W_{\omega}^{\mathrm{rad}}(z)}{\partial z}\right|_{z=0} C_{2}, \\
\text { where } \quad C_{i} \equiv \int_{0}^{\pi / 2} R(\theta) \cos ^{i} \theta \sin \theta \mathrm{d} \theta .
\end{gathered}
$$

Eq. 4.12 gives a similar mixed boundary condition as Eqs. 4.7 and 4.8, where the average reflectivity coefficient is now explicit $[128,159]$ :

$$
\bar{R} \equiv \frac{3 C_{2}+2 C_{1}}{3 C_{2}-2 C_{1}+2} .
$$

The angular reflection $R(\theta)$ is the average over polarization of the Fresnel coefficients since multiple scattering randomizes the polarization [160]. It is also possible to consider several interfaces. In the case of a sample with an extra transparent layer on top of the diffusive material, the extra reflections can be considered thanks to geometrical optics, according to [159]

$$
R(\theta)=\frac{R_{a b}+R_{b c}-2 R_{a b} R_{b c}}{1-R_{a b} R_{b c}},
$$

where $R_{i j}$ denotes the angular-dependent reflection coefficient between materials ${ }^{2} i$ and $j$.

\subsubsection{Angular-resolved diffuse transmission}

The extrapolation ratio $\tau_{\mathrm{e}}$ is a very important parameter in the analysis of diffusion measurements, as is obvious from previously derived diffuse total transmission (Eq. 2.62) and EBS cone (Eqs. 2.80 and 2.81). The extrapolation ratio modifies the width of the EBS cone (Eq. 2.83) although has a negligible effect on the EBS shape. The diffuse transmission, stationary or dynamic, is seen (Eqs. 2.62 and 2.66) to depend on the extrapolated thickness, $L_{\mathrm{ex}} \equiv L+\ell\left(\tau_{\mathrm{e}_{1}}+\tau_{\mathrm{e}_{2}}\right)$. An unknown, or badly estimated, extrapolation ratio can easily lead to an underestimation of $L_{\mathrm{ex}}$, more particularly noticeable for samples of thickness close to the transport mean free path. Following measurements (see Fig. 4.4) show that the extrapolation ratio can be greater than 10 , and can therefore hardly be considered as a small correction.

According to Eqs. 4.8 and 4.14 . $\tau_{\mathrm{e}}$ depends on the Fresnel coefficients and therefore on the refractive index of the diffusive material. The effective refractive index can be calculated according to theories [3] known as Lorentz-Lorenz [161], Maxwell Garnett [162]

\footnotetext{
${ }^{2}$ For example a colloidal suspension in a glass cell, in air: then $R_{a b}$ is the reflection at the interface air-glass, and $R_{b c}$ is the reflection at the interface glass-colloid suspension.
} 
or Bruggeman [163]. These effective medium theories consider various mixtures of two components with known dielectric functions and volume fractions, but in the large wavelength limit only. A more general, although numerical, approach lies in the so-called Energy Density Coherent Potential Approximation [164-166], which allows the calculation of the refractive index of a mixture of scatterers of known scattering properties. The samples which are used in this thesis and described in chapter 3 have pores of diameter smaller but comparable to the wavelength. Such porous structures also present ill-defined scatterers. Therefore porous $\mathrm{GaP}$ samples do not fulfill the assumptions for the effective medium theories nor EDCPA. Experimental determination of the refractive index is usually done by refractometry, interferometry [167] or speed-of-light measurement, all requiring a sufficiently transparent medium at the given wavelength. The angular-resolved diffuse transmission $[93,159]$ allows an experimental determination of the index of refraction in diffusive materials.

According to the boundary condition of Eq. 4.7, the energy density can be approximated, close to the boundary at $z=0$, by

$$
W_{\omega}^{\mathrm{rad}}(r, \theta, \phi)=\frac{W_{\omega}^{\mathrm{rad}}(r, \pi / 2, \phi)}{z_{\mathrm{e}}}\left(r \cos \theta+z_{\mathrm{e}}\right)
$$

The flux just before leaving the diffusive material can be calculated thanks to Eq. 4.3 integrated over $\mathrm{d} r$ and $\mathrm{d} \phi$, and within the approximation of Eq. 4.16 .

$$
\mathrm{d} J^{-}(\theta)=\frac{\nu_{\mathrm{E}}}{z_{\mathrm{e}}} W_{\omega}^{\mathrm{rad}}(r, \pi / 2, \phi)\left(\ell \cos \theta+z_{\mathrm{e}}\right) \cos \theta \sin \theta \mathrm{d} \theta .
$$

By crossing the interface, refraction from $\theta$ to $\theta_{\mathrm{e}}$ according to Snell's law, and reflection according to the Fresnel coefficient $R(\theta)$ will occur. Denoting $\mu \equiv \cos \theta$ and $\mu_{\mathrm{e}} \equiv \cos \theta_{\mathrm{e}}$, the escape function $E\left(\mu_{\mathrm{e}}\right)$, or the probability of light escaping the medium at an angle $\mu_{\mathrm{e}}$, is [159]

$$
\frac{E\left(\mu_{\mathrm{e}}\right)}{\mu_{\mathrm{e}}} \propto\left(\tau_{\mathrm{e}}+\mu\right)[1-R(\mu)]
$$

The proportionality constant is set by the normalization of this probability function. The reflection coefficient $R(\mu)$ is polarization-dependent, and gives two independent escape functions, for polarization in and out of the incidence plane. In the case of non-polarized light, the proportionality constant of Eq. 4.18 can be explicitly calculated [159] as $\left(3 n_{\mathrm{e}}^{2} / 2 n_{0}^{2}\right)$, where $n_{\mathrm{e}}$ and $n_{0}$ are the refractive index of the diffusive material and the surrounding medium, respectively. This escape function model can be generalized to other reflectivity functions than derived by Fresnel, and has been successfully applied to photonic crystals $[45,168,169]$.

The extrapolation ratio $\tau_{\mathrm{e}}$ depending only on the assumed reflectivity function $R(\mu)$ (see Eqs. 4.8 and 4.14, the escape function in Eq. 4.18 only depends on a single parameter, the effective refractive index of the diffusive material. 


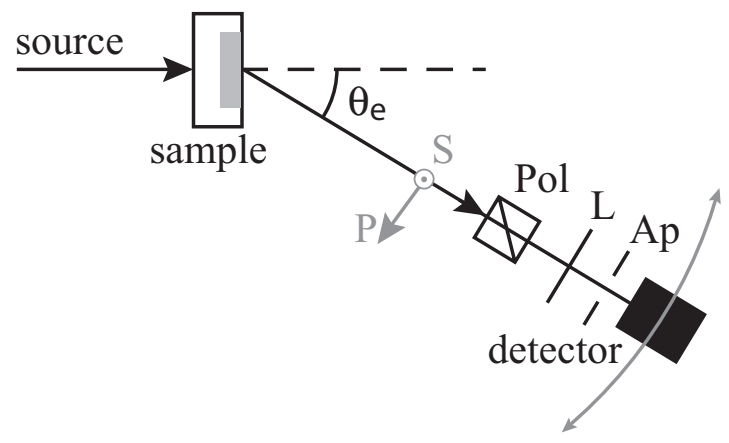

Figure 4.2: Cartoon of the angularresolved transmission setup. Diffuse light transmitted through the sample is focussed on a detector via a lens $(L)$. An aperture (Ap) reduces stray light. A polarizer (Pol) allows the measurement of one of the two polarizations, in $(P)$ or out $(S)$ of plane. The escape function thus measured allows the determination of the effective refractive index of the diffusive sample.

\subsection{Measuring the index of refraction of porous media}

\subsubsection{Experimental setup}

The setup used to measure the angular-resolved transmission (ART) [93] through diffusive samples is sketched in Fig. 4.2. A collimated beam from a helium neon laser $(633 \mathrm{~nm})$ impinges on the front side of the sample, where the GaP wafer remains. The incident polarization is not an issue for the diffuse transmission since after a few transport mean free paths the polarization of diffuse light is scrambled. The coherent transmission and single scattered light, if measurable in transmission, mainly retain the incident polarization. The $\mathrm{P}$ polarization (electrical field in the plane of incidence) has more features than the $\mathrm{S}$ polarization (electrical field normal to the incidence plane), such as the Brewster angle. It is therefore preferable to choose the incident polarization as S. The scattered light in transmission from the sample is collected by a lens $(f=10 \mathrm{~cm})$ at a distance of $90 \mathrm{~cm}$ from the sample. The intensity of the scattered light is measured by a silicon photodiode in the focal plane of the lens. Both polarizations of the scattered light are recorded, one after the other, thanks to a polarizer. An aperture is used in front of the detector to reduce stray light and background intensity. The combination of a chopper, modulating the intensity of the incident beam, and a lock-in amplifier further reduces the noise and the undesired background of the measurement. The number of speckle spots falling on the detector in the stationary setup is approximately 300. Further speckle averaging is typically done by spinning the sample with the same axis as the incident beam, and integrating over time thanks to the lock-in amplifier. The detector and collection optics are mounted on a goniometer, with the sample at its center of rotation. The angular range of the goniometer is $180^{\circ}$. A small range of angles, at grazing incidence from the interface of the sample $\left(\theta_{\mathrm{e}} \approx \pi / 2\right.$ or $\left.\mu_{\mathrm{e}} \approx 0\right)$, is blocked by the mounting of the sample.

The ART setup is first checked on a known, weakly scattering, sample. A colloid suspension of polystyrene spheres in water, enclosed in a glass cell, is used. The low volume fraction of the spheres $(\approx 2 \%)$ and the index of refraction of polystyrene $(n=1.59)$ ensures that the effective refractive index of the colloid is very close to that of plain water, i.e., 1.33. The diameter of the spheres $(426 \mathrm{~nm})$ nonetheless induces a transport mean free path smaller than the cell thickness $(1 \mathrm{~mm})$, since the solution looks milky whit 3

\footnotetext{
${ }^{3}$ At least after sufficient shaking, to break the sedimentation of the spheres.
} 


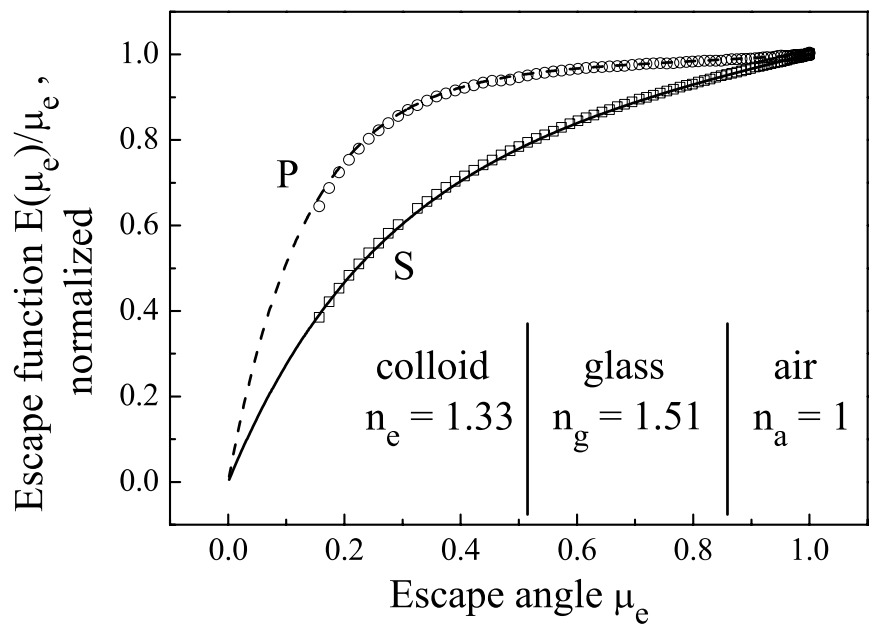

Figure 4.3: Escape function of a dilute colloid suspension in a glass cell. Measurements for both polarizations ( $P$ circles, $S$ squares) as a function of angle are presented. The theory of Eq. 4.18 for an effective refractive index of 1.33, covered by a layer of refractive index 1.51 is plotted, for both polarizations (P dashed curve, $S$ full curve). All curves are normalized to 1 at $\mu_{\mathrm{e}}=1$. The agreement between measurement and theory without adjusting parameters validates the ART method to determine the effective refractive index of diffusive material.

The Brownian motion of the colloid has a typical time scale of $1 \mathrm{~ms}$. Speckle is therefore averaged very quickly in a stationary measurement, with an integration time of the order of 1 second. The cell is made of BK7 glass, with a known refractive index $\left(n_{g}=1.51\right)$. The measured escape function for this colloid is shown in Fig. 4.3 .

The theoretical prediction for the escape function of this colloidal system is described in previous section. Eq. 4.18 describes the shape of the escape function for a single interface. Eq. 4.15 extends the model to a double-interface, as is needed in the case of the system colloid-glass-air. This extension relies on the series of multiple reflections within the middle layer (here, the glass), in a geometrical optics approach [159]. The only parameters of the model describing this colloid system are the three refractive indices, of colloid, glass and air, respectively $1.33,1.51$ and 1 . The theory, considering the two interfaces and the three known refractive indexes, is plotted in Fig. 4.3 without any free parameters. The very good agreement between measurements and theory for the colloidal system validates the use of the ART, along with the escape function theory in section 4.2.2 to determine the effective refractive index of diffusive material. Fig. 4.3 shows that geometrical optics is a good approximation for the two-interfaces escape function theory when the thickness of the extra layer is much larger than the wavelength.

\subsubsection{Measurements on strongly scattering samples}

The ART setup is used to determine the escape function of strongly scattering samples, made according to the methods described in chapter 3 A porous sample is prepared from a GaP wafer of (sulfur) dopant density $7 \times 10^{17} \mathrm{~cm}^{-3}$, anodically etched at $6 \mathrm{~V}$ in a solution 


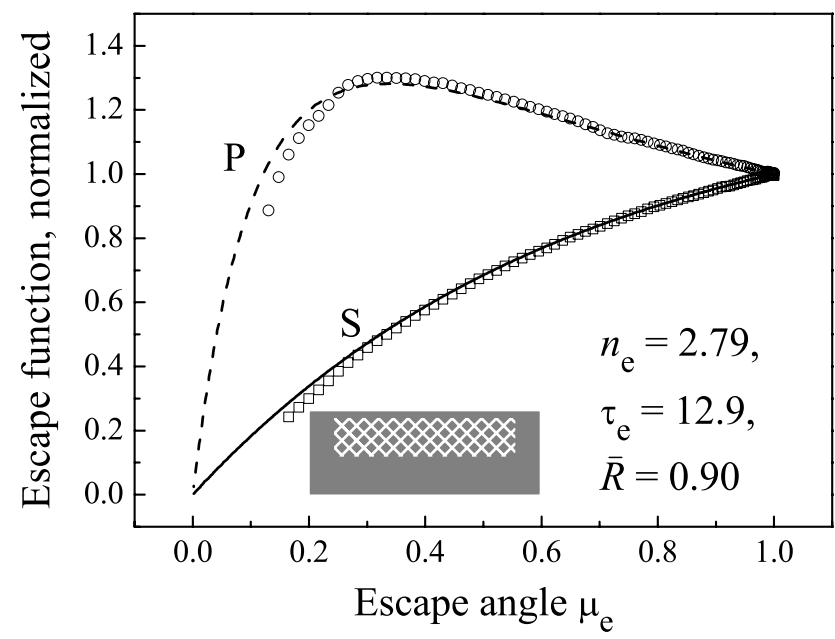

Figure 4.4: Escape function $E\left(\mu_{\mathrm{e}}\right) / \mu_{\mathrm{e}}$ of a porous GaP sample after anodic etching, for both polarizations (P circles, $S$ squares). The fit to the escape function theory gives an effective refractive index $n_{\mathrm{e}}=2.79$, or equivalently $\tau_{\mathrm{e}}$ or $\bar{R}$. The presence of the top-layer on the diffusive sample influences greatly the escape function, and leads to a wrong refractive index.

of $\mathrm{H}_{2} \mathrm{SO}_{4}, 0.5 \mathrm{~mol} / \mathrm{L}$. The thickness of the porous layer is $213 \pm 8 \mu \mathrm{m}$, and its porosity is $13 \%$.

This one, and typical, sample will be used as an example throughout the rest of present section. The escape function measurements on this sample are shown in Figs. 4.4, 4.5 and 4.6. respectively after anodic etching, photochemical etching and chemical etching. In each graph of these 3 figures, a cartoon of the cross section of the sample is shown, with and without top-layer, and with larger pores, respectively.

After anodic etching, porous $\mathrm{GaP}$ is diffusive and has a transparent top-layer. The porous layer is grown sufficiently thick so that the coherent transmission is negligible. On Fig. 4.4 the measurement of the porous sample still covered by the GaP top-layer is plotted, along with the fit to the escape function theory, Eq.4.18. The escape function fit considers one interface, between an average medium, of index $n_{\mathrm{e}}$, and air. The measurements for both polarizations on the anodically etched sample are well described by one single parameter, the effective refractive index, found as $n_{\mathrm{e}}=2.79$. Of course, this value can not be taken as the refractive index of the bulk porous material, since the top-layer influences the measurement. A high refractive index at the interface (such as 3.3 for the top-layer here) dominates the shape of the escape function. Therefore, it is not possible to reliably fit the measurements in Fig. 4.4 with the model with two interfaces (Eqs. 4.18 with 4.15) in order to obtain the refractive index of the bulk. Although the effective refractive index $n_{\mathrm{e}}=2.79$ does not contain useful information, the corresponding extrapolation ratio $\tau_{\mathrm{e}}$ and internal reflection coefficient $\bar{R}$ are relevant. It is possible to consider the fit to Eq. 4.18 as a fit on the extrapolation ratio, which is well defined even in a multiple-interfaces system. This determination of the extrapolation ratio allows further interpretation of optical measurements (like total transmission, Eq. 2.62, or EBS width, Eq. 2.83). The wave vector of light in 


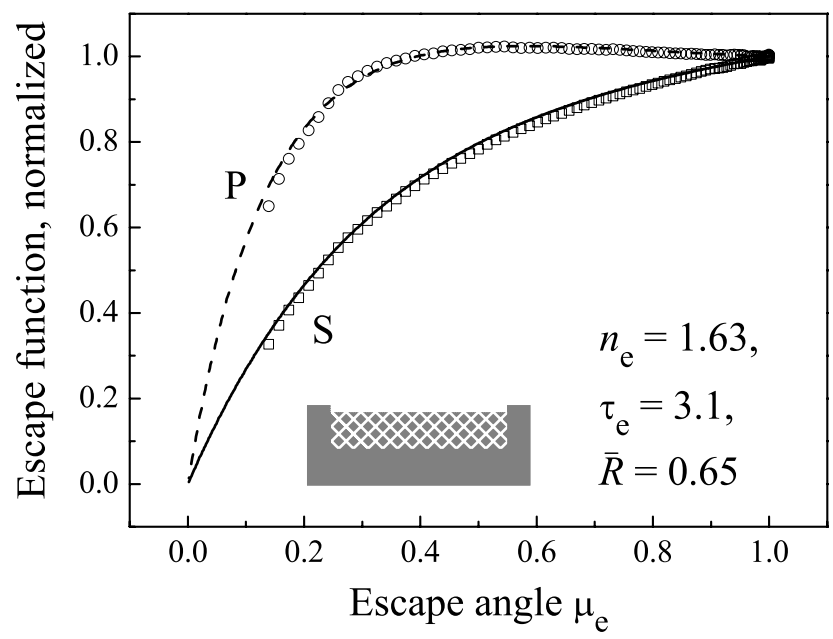

Figure 4.5: Escape function $E\left(\mu_{\mathrm{e}}\right) / \mu_{\mathrm{e}}$ of a porous GaP sample after anodic and photochemical etching, for both polarizations (P circles, $S$ squares). The fit to the escape function theory gives an effective refractive index $n_{\mathrm{e}}=1.63$. The measured refractive index is now from the bulk.

the material, necessary to infer the parameter $k \ell$, is still not known since it depends on the effective refractive index. A high value of the extrapolation ratio makes it dominate the interpretation of optical measurements, and a better solution for interpretation is to remove the top-layer.

From the measurement in Fig. 4.5 the refractive index of the bulk material of this sample is found as $n_{\mathrm{e}}=1.63$. Using the two-interfaces model of the escape function (see Eq. 4.15 and Fig. 4.3 for a system with a refractive index $n_{\mathrm{e}}=1.63$, a transparent top-layer of index $n=3.3$ and air does not give a good agreement with the measurements of Fig. 4.4. The $\mathrm{GaP}$ top-layer of anodically etched porous $\mathrm{GaP}$ is $200 \mathrm{~nm}$ thick. The geometrical optics derivation for the two-interfaces reflection coefficient (Eq. 4.15) does not apply to a layer of thickness comparable to the wavelength of light.

After photochemical etching, described in section 3.3.1, the top-layer is removed, without noticeably modifying the porous structure. In Fig. 4.5 , the ART measurements of the photochemically etched sample, along with the fit to the escape function theory with one interface, are plotted. The fit in Fig. 4.5 yields a different effective refractive index than the fit for the sample with top-layer, in Fig. 4.4. The effective refractive index of the photochemically sample is homogeneous, from the interface to the bulk. It can therefore be concluded that the value fitted here, $n_{\mathrm{e}}=1.63$, characterizes the bulk effective refractive index. Such a refractive index, of a sample after removal of the top-layer, is used to further interpret other optical measurements and to determine the wave vector of light in the material, as $k=n_{\mathrm{e}} k_{0}=2 \pi n_{\mathrm{e}} / \lambda_{0}$.

Performing further chemical etching (see section 3.3.2) on the previously photo-chemically etched sample increases the average diameter of the pores. In Fig. 4.6 are shown the escape function measurements on the sample after 10 hours of chemical etching, and the fit to the escape function theory with one interface. A good agreement between the 


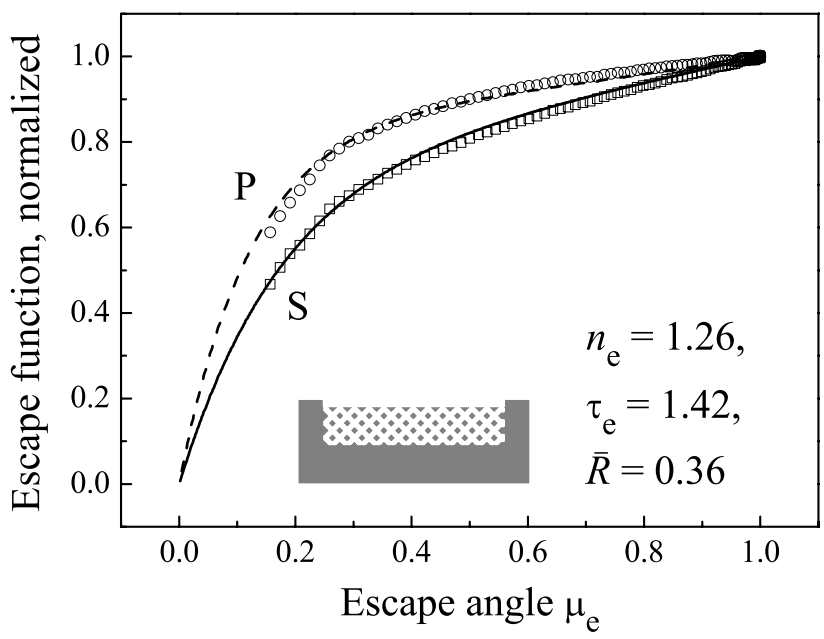

Figure 4.6: Escape function $E\left(\mu_{\mathrm{e}}\right) / \mu_{\mathrm{e}}$ of a porous GaP sample after anodic, photochemical, and chemical etching, for both polarizations ( $P$ circles, $S$ squares). The fit to the escape function theory gives an effective refractive index $n_{\mathrm{e}}=1.26$. The increase in porosity via chemical etching induces, as intuitively expected, a smaller refractive index.

escape function measurements and the theory for $n_{\mathrm{e}}=1.26$ is found. A lower value of the effective refractive index was intuitively expected since increasing the diameter of the pores increases porosity. Indeed, the porosity of the chemically-etched sample is estimated ${ }^{4}$ as $60 \%$. The following section takes a more systematic approach to determine the effective refractive index of samples in a range of different porosities.

The measurements presented in Figs. 4.4, 4.5 and 4.6 give confidence in the assumption that a Fresnel reflection coefficient can be used at the interface of the strongly scattering samples described here. A Fresnel coefficient follows from a flat interface between two dielectric materials. The diameter of the pores in anodically etched samples gives an estimation of the roughness of the interface of the porous region, i.e., in the range 40-200 nm. A flat interface means that the roughness at the interface is much smaller than the wavelength of the light. At $633 \mathrm{~nm}$, this flatness condition is mildly fulfilled. The good agreement between the ART measurements and the corresponding fits to the escape function theory shows this condition to be sufficiently fulfilled.

\subsection{Index of refraction versus porosity}

The combination of tunable (strong) scattering properties of a porous material $[81,84,170]$ and the ART measurements allows a study of the refractive index as a function of porosity in the strong scattering regime [93]. The structural (pore diameter, shape, inter-pore wall thickness) as well as the scattering (mean free path) properties of porous $\mathrm{GaP}$ depend on

\footnotetext{
${ }^{4}$ Contrarily to the porosity of the sample after anodic etching, which can be determined thanks to the total charge etched [151], the porosity after chemical etching can not be reliably determined. Weighting the sample before and after chemical etching is a good, although inaccurate, way to obtain an estimation.
} 


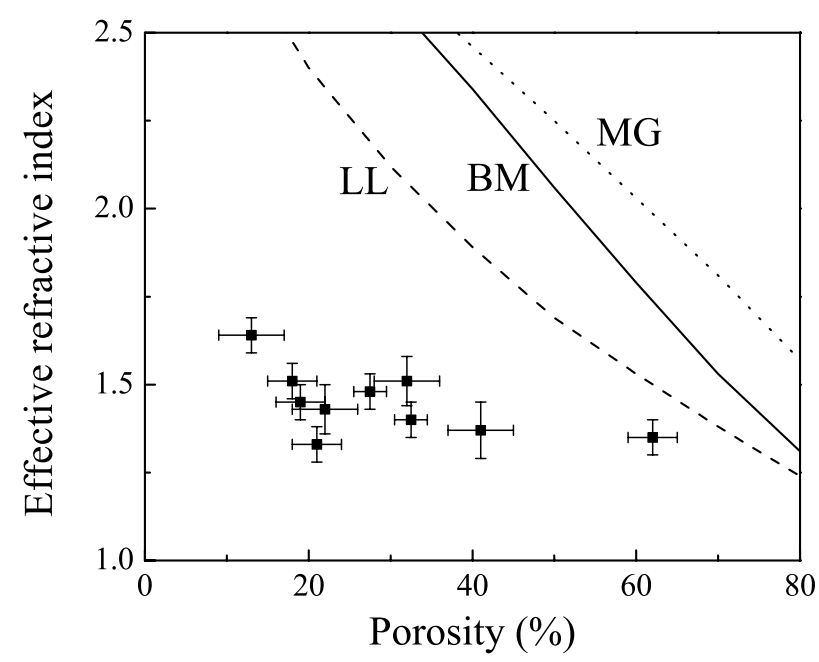

Figure 4.7: Effective refractive index of porous GaP as a function of the porosity of strongly scattering samples. A higher porosity implies a smaller proportion of the high-index material, GaP. For comparison, the three effective medium theories are plotted, but fail to describe strongly scattering porous GaP.

the exact conditions of the anodic etching step. As was described in section 3.2, mainly the doping concentration of the $\mathrm{GaP}$ wafer and the etching potential influence the formation of the porous structure. In the scope of this section, porous GaP samples from two different wafers, etched in $0.5 \mathrm{~mol} / \mathrm{L}$ sulfuric acid at various potentials, are considered. In order to obtain the bulk refractive index of the material, only samples after photochemical etching are used, i.e., after removal of the top-layer. A series of samples of porosity ranging from $13 \%$ to $62 \%$ was made. A lower porosity is phenomenologically obtained by decreasing the etching potential. The lower limit to the porosity is set by practicality. At low etching potential, the speed of etching is very small, and a sufficiently thick porous sample is very time-consuming to produce. A higher porosity can be obtained by chemical etching for example (see section 3.3.2). The porosity after chemical etching is not well known, and corresponding measurements are therefore not presented. The porosity of each sample is determined thanks to the total charge during etching [151]. The ART of each sample is measured, and fitted to the escape function theory to obtain the bulk refractive index. The effective refractive indices are plotted as a function of porosity in Fig. 4.7. Only for a porosity of $0 \%$ or $100 \%$ is the effective refractive index a priori known, since it reaches the bulk value of the constituents, $\mathrm{GaP}$ and air ( $n=3.3$ and $n=1$ respectively). The measurements in Fig. 4.7 show a trend of decreasing refractive index for increasing porosity, as intuitively expected. A useful comparison lies in the effective medium theories, derived by Lorentz-Lorenz (LL) [161], Maxwell Garnett (MG) [162] and Bruggeman (BM) [163]. The LL approach considers a mixture of spheres of two different materials embedded in vacuum. The MG approach considers spheres of a material embedded in a matrix of another material. The BM approach considers a mixture of spheres of different materials embedded in the effective medium itself. 


\begin{tabular}{|c|c||c|c|c|c|c|c|}
\hline $\begin{array}{c}\text { wafer } \\
\text { doping }\end{array}$ & $\begin{array}{c}V_{\text {etch }} \\
(\mathrm{V})\end{array}$ & $\begin{array}{c}\varphi \\
(\%)\end{array}$ & $\begin{array}{c}d_{\text {pores }} \\
(\mathrm{nm})\end{array}$ & $\begin{array}{c}d_{\text {wall }} \\
(\mathrm{nm})\end{array}$ & $\begin{array}{c}\ell \\
(\mu \mathrm{m})\end{array}$ & $n_{\mathrm{e}}$ & $\tau_{\mathrm{e}}$ \\
\hline \hline & 6 & $13 \pm 4$ & $44 \pm 13$ & $104 \pm 21$ & $0.97 \pm .08$ & $1.64 \pm .05$ & $3.13 \pm .31$ \\
& 7 & $18 \pm 3$ & $43 \pm 11$ & $161 \pm 96$ & $0.84 \pm .08$ & $1.51 \pm .05$ & $2.47 \pm .24$ \\
$7 \times 10^{17}$ & 8 & $19 \pm 3$ & $47 \pm 19$ & $180 \pm 82$ & $0.91 \pm .01$ & $1.45 \pm .05$ & $2.20 \pm .22$ \\
$\left(\mathrm{~cm}^{-3}\right)$ & 9 & $22 \pm 4$ & $56 \pm 11$ & $199 \pm 100$ & $0.98 \pm .10$ & $1.43 \pm .07$ & $2.11 \pm .30$ \\
& 9.5 & $28 \pm 2$ & $64 \pm 14$ & $140 \pm 56$ & $1.17 \pm .12$ & $1.48 \pm .05$ & $2.33 \pm .25$ \\
& 10.5 & $33 \pm 2$ & $72 \pm 21$ & $126 \pm 59$ & $1.28 \pm .12$ & $1.40 \pm .05$ & $1.97 \pm .22$ \\
& 11.2 & $62 \pm 3$ & $61 \pm 90$ & $50 \pm 70$ & $1.16 \pm .10$ & $1.35 \pm .05$ & $1.76 \pm .20$ \\
\hline $6 \times 10^{17}$ & 13 & $21 \pm 3$ & $96 \pm 28$ & $152 \pm 80$ & $0.67 \pm .08$ & $1.33 \pm .05$ & $1.69 \pm .20$ \\
$\left(\mathrm{~cm}^{-3}\right)$ & 14 & $41 \pm 4$ & $93 \pm 27$ & $107 \pm 46$ & $0.83 \pm .09$ & $1.37 \pm .08$ & $1.86 \pm .35$ \\
\hline
\end{tabular}

Table 4.1: Porosity $\varphi$, pore diameter $d_{\text {pores }}$, inter-pore wall thickness $d_{\text {wall }}$, transport mean free path $\ell$, refractive index $n_{\mathrm{e}}$ and extrapolation ratio $\tau_{\mathrm{e}}$ of samples from wafers of two different doping concentration and etched at different potentials $V_{\text {etch. }}$.

The three approaches (LL, MG and BM) can be summarized in one equation [171]:

$$
\frac{\varepsilon_{\mathrm{e}}-\varepsilon_{\mathrm{h}}}{\varepsilon_{\mathrm{e}}+2 \varepsilon_{\mathrm{h}}}=\varphi_{1} \frac{\varepsilon_{1}-\varepsilon_{\mathrm{h}}}{\varepsilon_{1}+2 \varepsilon_{\mathrm{h}}}+\varphi_{2} \frac{\varepsilon_{2}-\varepsilon_{\mathrm{h}}}{\varepsilon_{2}+2 \varepsilon_{\mathrm{h}}}
$$

where $\varphi_{i}$ and $\varepsilon_{i}$ are respectively the porosity and dielectric constant of material $i, \varepsilon_{\mathrm{e}} \equiv n_{\mathrm{e}}^{2}$ is the effective dielectric constant of the mixture, and $\varepsilon_{\mathrm{h}}$ is the dielectric constant of the host material, dependent on the approach. In the LL case, the host material is vacuum. In the MG case, the host is one of the two materials. In the BM case, the host is the effective medium itself. The MG approach is asymmetric since it requires to choose one of the two materials as the host. If one of the two materials is vacuum, the MG case with vacuum as host and LL case are equal. In Fig. 4.7 the MG case with the GaP as host is plotted. To this day, the comparison of experimental data to effective medium theories failed to show any systematic agreement with any theory [3]. Granular metals or semiconductors, where nanometer-sized grains are embedded in a continuous matrix, have been shown to follow the MG predictions [172]. On the other hand, the conductivity of binary metallic mixtures, where the two components are equivalent in the mixture, can better be described by the BM approach [173].

Porous GaP can not be described by inclusions of one material (e.g., air) in the other (e.g., $\mathrm{GaP}$ ) since both materials are interconnected on a large scale. The GaP stays monocrystalline, and one pore extends from one pit at the surface up to the bottom of the porous layer. The BM approach is thus expected to describe the effective refractive index of porous GaP. From Fig. 4.7, it is obvious that no effective medium theory can account for the refractive index of strongly scattering porous GaP. In table 4.1, several structural and diffusion parameters of the samples from Fig. 4.7 are shown. SEM images of the bulk of each sample allows the measurement of the average and standard deviation values of the pore diameter $d_{\text {pores }}$ and the inter-pore wall thickness $d_{\text {wall }}$. The pore diameter is the length scale of the air regions, whereas the wall thickness is the length scale of the GaP regions. The transport mean free path $\ell$ is deduced from EBS measurements, taking into account the measured extrapolation ratio $\tau_{\mathrm{e}}$. The length scale of the inhomogeneity in porous GaP ranges from 
$40 \mathrm{~nm}$ to $200 \mathrm{~nm}$. Porous $\mathrm{GaP}$ at $633 \mathrm{~nm}$ hardly fulfills the long wavelength limit of the effective medium theories.

The effective medium theories calculate an effective refractive index from the phase velocity in the composite material. The phase velocity is a field quantity, i.e., it is theoretically found by using field Green functions (see Eq.2.23). The escape function theory used here to determine the refractive index relies on the diffusion approximation. Diffusion is a phenomenon which is characteristic of intensity, as is obvious from section 2.4. It is yet to be proven that the effective refractive index of a diffusive material, determined by the escape function theory, is the only refractive index. The fact that the escape function theory shows very good agreement with ART measurements in porous GaP does not imply that the measured effective refractive index is related to the phase velocity in the material [3]. The refractive indices shown in this section are phenomenological, and only describe, through the escape function theory, the ART measurements. It is therefore a bold step to consider that the calculation of the effective medium theories apply to the properties of a diffusive medium. In this thesis, the effective refractive index, determined from ART measurements, is only used to further interpretation of other diffusion experiments. Within the diffusion regime the refractive index, or more importantly the extrapolation ratio, stays consistent and unique.

\subsection{Conclusions}

The proper treatment of a diffusive medium of finite size must include boundary conditions. The characteristic property of an interface, for diffusion, is the extrapolation ratio, which depends solely on the internal reflectivity at the interface. The angular-resolved intensity of light escaping a diffusive material was shown to depend on the extrapolation ratio. Performing an escape function measurement allows the determination of the extrapolation ratio, and therefore the effective refractive index, of strongly scattering samples, produced by electro- and photochemical etching of GaP. From the escape measurements of a range of porous samples, the refractive index as a function of porosity was determined. In the regime of strong scattering, the refractive index does not follow the usual effectivemedium theories. 



\section{Chapter 5}

\section{Strong scattering in porous GaP}

\subsection{Introduction}

Anderson localization of light is a phase transition induced by disorder [19]. In 1D and 2D systems, light is always localized in a disordered material where the coherence length is long enough $[22,23]$. The coherence length is the extension of the disordered material in which interference effects are present. The finite thickness and absorption of the material are the two principal effects limiting the coherence in a disordered material. Localization of light has been clearly observed in 1D and 2D systems [29, 64, 174-177].

In $3 \mathrm{D}$ systems, the localization transition is expected to happen for $k \ell_{\mathrm{B}} \simeq 1$, the socalled Ioffe-Regel criterion [24], where $k$ is the wave vector of light, and $\ell_{\mathrm{B}} \equiv \ell_{\mathrm{sc}} /(1-\langle\cos \theta\rangle)$ the Boltzmann mean free path in the material. Although 3D Anderson localization has been extensively searched for, it remains elusive. Several claims for 3D Anderson localization for light in particular have been filed. The low diffusion constant in samples of $\mathrm{TiO}_{2}$ scatterers, initially attributed to localization [72], was later found to be due to resonant scattering [178]. A glass phase of aluminium and Teflon spheres was shown to localize microwaves [27], although the strong absorption in this sample complicated the analysis of the measurements. Localization of near-infrared light was reported [76], following the analysis of the EBS cone and total transmission through gallium arsenide powders. This semiconductor powder was suspected to display absorption [138, 179], which would invalidate the claim of Anderson localization in reference [76]. The rounding of the EBS cone at visible wavelength from porous $\mathrm{GaP}$ samples was interpreted [83] as the onset of Anderson localization. Optical absorption was shown to be small enough [82] in porous $\mathrm{GaP}$ to have no influence on the EBS measurements.

There is an ambiguity in the analysis of total transmission and EBS measurements, since the same outcome of one of these measurements can be interpreted either in terms of absorption or localization. The careful study of the fluctuations of the transmitted flux through a multiple-scattering medium is expected [30] to discriminate between localization and absorption. Such study of fluctuations is not done in the present thesis.

In chapter 3 , the formation methods of porous $\mathrm{GaP}$ are described and explained. Anodic etching (see section 3.2 allows a layer of strongly scattering porous material to be made from a GaP wafer. Photochemical etching (see section 3.3.1 removes the bare GaP 
top-layer, remaining from the anodic etching, which simplifies the analysis of optical measurements. Further chemical etching increases the size of the pores, to obtain stronger scattering (see section 3.3.2).

The first study $[82,83]$ of the scattering properties of porous GaP was done on two kinds of samples: anodically etched (A-GaP) and 'photo-anodically' etched (PA-GaP). The original $\mathrm{GaP}$ wafers used to produce the porous samples were doped with sulfur, with a doping concentration $N=2 \times 10^{17} \mathrm{~cm}^{-3}$, and a (100) surface orientation. GaP wafers of the same characteristics are not available anymore. The A-GaP samples are produced according to the same method as the samples presented in this thesis, at a constant potential of $15 \mathrm{~V}$. The PA-GaP samples are initially A-GaP samples, further photochemically etched, in a solution of $\mathrm{H}_{2} \mathrm{SO}_{4}$ and $\mathrm{H}_{2} \mathrm{O}_{2}$, under the illumination of a helium neon $(\mathrm{HeNe})$ laser $(50 \mathrm{~mW}$ at $633 \mathrm{~nm}$ ). The photo-anodic method of references [82,83] has two simultaneous effects: the increase of the pore size $e^{1}$ and the removal of the top-layer. The average pore diameter of the porous structure was increased, after photo-anodic etching, from $90 \mathrm{~nm}$ in A-GaP to $130 \mathrm{~nm}$ in PA-GaP. The surface of the PA-GaP samples does not display the strong specular reflection typical of the bare GaP top-layer anymore. The photo-anodic etching is equivalent, although in a less controlled way, to the two further, photochemical and chemical, etching steps described in sections 3.3.1 and 3.3.2 Provided the same original GaP wafers of reference [82,83] are available, samples equivalent to PA-GaP can be produced by following the etching steps described in chapter 3 . A second, more systematic, study of the scattering properties of porous $\mathrm{GaP}[81,84]$ failed to reproduce samples which display an Anderson localization effect. The present chapter is a continuation of this earlier systematic study.

In this chapter, the scattering properties of the porous GaP samples, produced according to the etching methods described in chapter 3, are studied. Samples from a wide range of doping concentration, etching potentials, and etching solutions are studied. The effect on the scattering properties of porous GaP of the two further, photochemical and chemical, etching steps is presented. No deviation from classical diffusion has been observed in the samples presented here. The most strongly scattering sample used in this thesis is made from a GaP wafer of doping concentration $N=5 \times 10^{17} \mathrm{~cm}^{-3}$, etched at $15.1 \mathrm{~V}$ in sulfuric acid, where $k \ell \simeq 3.5$ at a wavelength of $633 \mathrm{~nm}$.

In the following section, the scattering properties of anodically-etched samples are presented, with a focus on the effect of the bare GaP top-layer, the optical absorption, and the best scattering strength obtainable for samples of given doping concentration. The last section presents results on further chemically-etched samples, to increase the scattering strength of the porous structure.

\subsection{Anodically etched samples}

Porous GaP samples are made according to the anodic etching presented in section 3.2. The EBS from the porous samples is measured using the off-centered rotation technique [129] and a HeNe laser as light source, with a wavelength of $633 \mathrm{~nm}$ (See section 2.6 for the

\footnotetext{
${ }^{1}$ The incident beam at $633 \mathrm{~nm}$ is only absorbed via two-photon absorption in GaP. The holes generated by absorption of the incident light are therefore homogeneously distributed in the whole porous structure. The subsequent etching is therefore also homogeneous.
} 


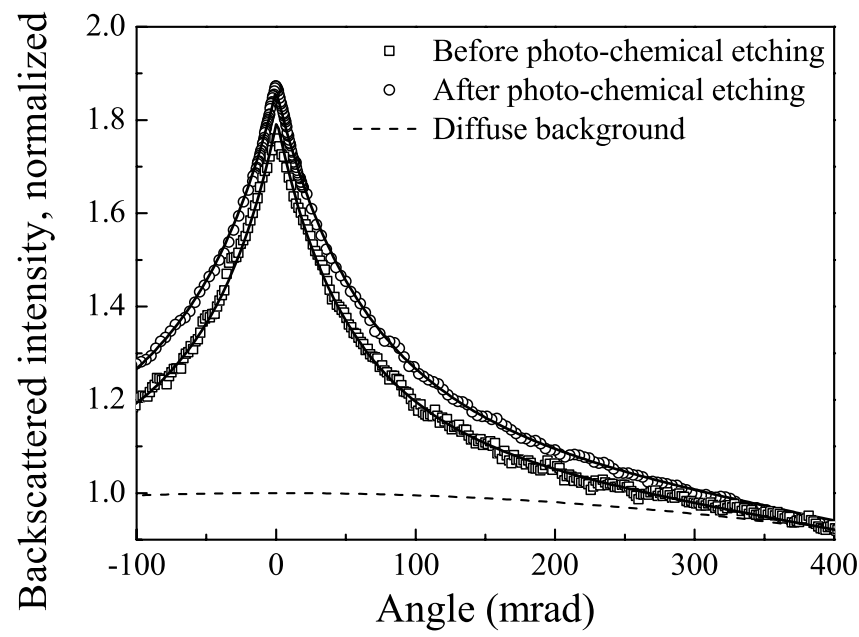

Figure 5.1: Backscattered intensity as a function of angle, normalized to the diffuse background (in dashed curve) at $O^{\circ}$. The porous sample has a doping concentration $N=5 \times 10^{17} \mathrm{~cm}^{-3}$, and was anodically etched at $14 \mathrm{~V}$. The EBS cones from a sample before (squares) and after (circles) photochemical etching, i.e., with and without the top-layer, are shown. Removing the top-layer has a large effect on the width of the EBS cone.

theory of the EBS). The same EBS setup is used in section 6.5.1 The samples are spun along an axis normal to their surface, to perform the ensemble averaging, and average the speckles out.

The EBS cone from a typical anodically-etched sample $\left(N=5 \times 10^{17} \mathrm{~cm}^{-3}\right.$, etched at $14 \mathrm{~V}$ in sulfuric acid) is plotted as squares in Fig. 5.1. The dashed curve in Fig. 5.1 is the Lambertian diffuse background (see Eq. 2.80). The EBS cone is fitted to the theory from section 2.6 (see Eqs. 2.80 and 2.81). If the effect of the internal reflection is not taken into account, i.e., for $\tau_{\mathrm{e}}=2 / 3$, the width of EBS cone gives a wrong value of the transport mean free path as $\ell\left(\tau_{\mathrm{e}}=2 / 3\right)=(0.74 \pm 0.06) \mu \mathrm{m}$. Measuring the escape function (see chapter 4) allows the determination of the extrapolation ratio of the sample. Fitting the same EBS measurement (squares in Fig. 5.1) by considering the experimentally-determined extrapolation ratio gives $\ell\left(\tau_{\mathrm{e}}=2.5\right)=(0.43 \pm 0.04) \mu \mathrm{m}$. The knowledge of the extrapolation ratio is very important to the determination of the transport mean free path.

\subsubsection{Photochemically etched samples}

The anodically-etched samples still have a highly-reflecting top-layer, which can be removed by photochemical etching. In section 4.3.2 the top-layer is shown to strongly modify the angular distribution of light escaping the diffusive medium. The anodically-etched porous samples are further photochemically etched (See section 3.3.1) in order to remove the top-layer. In Fig. 5.1. the EBS cone from the same sample, before (squares) and after (circles) photochemical etching, is shown. The removal of the top-layer decreases the internal reflection at the interface, and therefore increases the width of the EBS cone for 

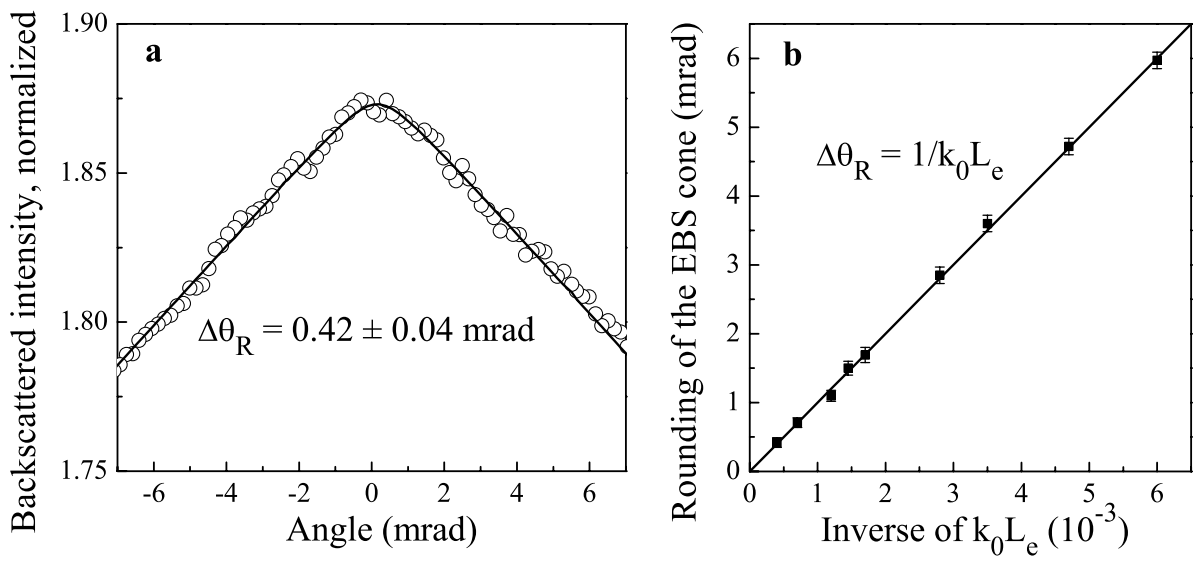

Figure 5.2: In (a), top of the EBS cone of a porous GaP sample $\left(N=5 \times 10^{17} \mathrm{~cm}^{-3}\right.$, etched at $\left.14 \mathrm{~V}\right)$, of thickness $L=248 \mu \mathrm{m}$. The fit (solid curve) gives the range of angles $\Delta \theta_{\mathrm{R}}$ in which the EBS is rounded. In (b), $\Delta \theta_{\mathrm{R}}$ as a function of thickness for a range of samples as in $(a)$. The solid curve is the expectation for $\Delta \theta_{\mathrm{R}}$ in the absence of absorption. The rounding of the EBS cone is only due to the finite thickness of the sample.

the same transport mean free path. The extrapolation ratio of the photochemically-etched sample is again determined by an escape function measurement. Fitting the EBS cone of the sample after photochemical etching, knowing the extrapolation ratio, gives the value for the transport mean free path $\ell\left(\tau_{\mathrm{e}}=1.6\right)=(0.42 \pm 0.02) \mu \mathrm{m}$. The transport mean free path of the sample is not changed, within experimental accuracy, by the photochemical etching. The photochemical etching removes the top-layer, but does not change the porous structure itself. Note that the experimental accuracy on the determination of the mean free path by EBS is increased when the top-layer is removed. In following samples, the top-layer is removed before performing any optical measurements.

\subsubsection{Absorption in porous GaP}

Absorption is a very important parameter to control during the etching processes of $\mathrm{GaP}$. $\mathrm{GaP}$ has negligible absorption for light at $633 \mathrm{~nm}$. In order to test the absorption of porous GaP samples, the shape of the EBS cone at small angles is investigated. In Fig. 5.2 the top of the EBS cone from the photochemically-etched sample (circles) in Fig. 5.1 is enlarged. This sample has a thickness $L=248 \mu \mathrm{m}$, determined from a SEM picture. The top of the EBS cone is rounded, at a typical angle $\Delta \theta_{\mathrm{R}}$. In Fig. $5.2 \mathrm{p}$, the rounding angle $\Delta \theta_{\mathrm{R}}$ is plotted for a range of samples identical to the one in Fig. 5.2 a, but with varying thickness. According to Eq. 2.84 the rounding of the EBS cone only due to finite thickness is inversely proportional to the thickness. In the case of $L_{\mathrm{e}} \equiv L+2 \tau_{\mathrm{e}} \ell=250 \mu \mathrm{m}$ the EBS rounding is expected as $\Delta \theta_{\mathrm{R}}=0.4 \mathrm{mrad}$. Within the experimental accuracy, the rounding of the EBS cone in Fig. $5.2 \mathrm{a}$ is only due to the finite size effect. The absorption length in this sample is therefore bigger than its thickness, $L_{\mathrm{a}}>250 \mu \mathrm{m}$. Anodic and photochemical etching do not introduce any measurable absorption, according to the rounding of the EBS cone. 

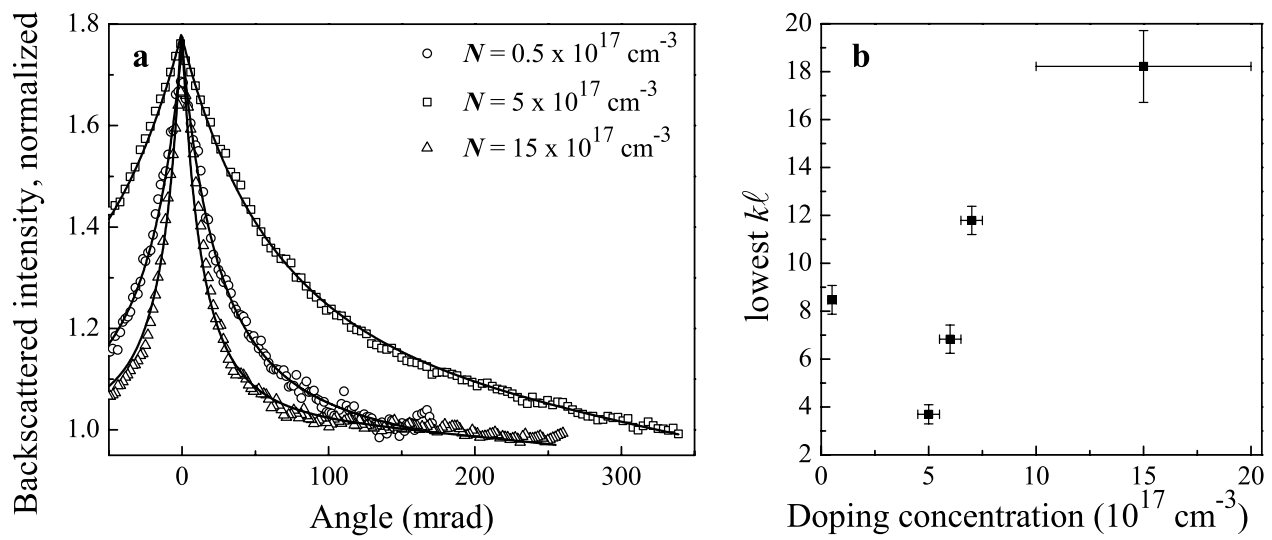

Figure 5.3: In (a), EBS measurements from porous GaP samples of various doping concentrations, etched at $V_{\max }$ in sulfuric acid. The solid curves are fits to the theory of section 2.6. In (b), lowest $k \ell$ parameter obtained for samples from wafers of different doping concentrations. The most strongly scattering samples are made from wafers with $N=2-5 \times 10^{17} \mathrm{~cm}^{-3}$.

\subsubsection{Doping concentration dependence}

In order to look for the strongest scattering in porous GaP, samples made from wafers of different doping concentrations, etched in different solutions and at different potentials, were studied. As mentioned in section 3.2 using nitric acid or phosphoric acid instead of sulfuric acid as the etching reagent does not produce a broader range of useable porous structures [153]. For a given GaP wafer and etching solution, the porous samples are more strongly scattering when etched at $V_{\max }$. Etching at a higher potential leads to a thicker depletion layer (see Eq. 3.2), to an increase of the average size of the pores toward the wavelength of light (see Fig. 3.6) and therefore to a more efficient scattering of the pores $[81,84]$. The dependence of the scattering strength on the doping concentration of the wafer is illustrated in Figs. 5.3 and 5.3 $\mathrm{p}$.

In Fig. 5.3 a, the EBS measurements from porous GaP samples of different doping concentration, etched at $V_{\max }$ in sulfuric acid, are shown. At a constant extrapolation ratio, a wider EBS cone implies a smaller transport mean free path in the material. The width of the EBS cone varies strongly with doping concentration.

In Fig. 5.3 b, a summary of the best achievable scattering strength from porous $\mathrm{GaP}$ samples of different concentrations is plotted. The parameter $k \ell=2 \pi n_{\mathrm{e}} \ell / \lambda_{0}$ represents the inverse of the scattering strength, where $\ell$ is obtained from the EBS cone, $n_{\mathrm{e}}$ from the escape function and $\lambda_{0}=633 \mathrm{~nm}$. In previous studies [81, 82,84], the highest scattering strength was shown to be obtained from samples of doping concentration lower or equal to $5 \times 10^{17} \mathrm{~cm}^{-3}$. The further decrease of the doping concentration does not, contrarily to what was expected $[81,84]$, allow the formation of a more strongly-scattering sample. The $k \ell$ curve in Fig. $5.3 \mathrm{p}$ has a minimum in the range $2-5 \times 10^{17} \mathrm{~cm}^{-3}$. The lack of tunability of the doping concentration of $\mathrm{GaP}$ wafers hampers the optimization of the scattering strength of porous $\mathrm{GaP}$ samples. 


\subsection{Increasing the pore size with chemical etching}

Both previous studies [81-83] of the scattering properties of porous GaP showed a decrease of the transport mean free path with chemical etching. In the first study $[82,83]$, "photoanodic' etching was used on a GaP wafer with $N=2 \times 10^{17} \mathrm{~cm}^{-3}$ to produce PA-GaP, where $k \ell \simeq 3$. Photo-anodic etching increases homogeneously the diameter of the pores, like chemical etching does (see section 3.3.2). In the second study [81], the strongest scattering sample, with $k \ell \simeq 3.5$, was made by anodic etching at $16.6 \mathrm{~V}$ of a GaP wafer with $N=5 \times 10^{17} \mathrm{~cm}^{-3}$. After further chemical etching, this anodically-etched sample displayed a smaller in situ total transmission (where the sample was still filled with water) but a narrower EBS cone. Neither of these two samples can be exactly reproduced due to the lack of equivalent $\mathrm{GaP}$ wafers. The doping concentration of wafers available, and studied in this thesis, include $N=5 \times 10^{17} \mathrm{~cm}^{-3}$, quoted by the supplier. The potential of maximum etching current $V_{\max }$ is different for this available wafer than for the wafer of same, quoted, doping concentration in reference [81]. The potential of highest etching current $V_{\max }$ being a characteristic of the wafer doping concentration and the etching solution, two wafers can be discriminated with the help of the determination of $V_{\max }$.

\subsubsection{Increasing the scattering strength}

In order to test the increase in scattering strength of chemically-etched samples, the following experiment was performed. A range of samples from a wafer of doping concentration $N=5 \times 10^{17} \mathrm{~cm}^{-3}$, anodically etched at $14.5 \mathrm{~V}$ in sulfuric acid, and with various thicknesses $(30-280 \mu \mathrm{m})$ is prepared. After photochemical etching, to remove the bare $\mathrm{GaP}$ top-layer, the range of samples is characterized by escape function (see section 4.3), total transmission (see section 2.5) and EBS (see section 2.5.1) measurements. The samples are then chemically etched for $\simeq 4300 \mathrm{~s}$ each. A second chemical etching step is made, until almost the minimum in in situ transmission. After each of the two chemical-etching steps, escape function, total transmission and EBS measurements are again performed on the samples.

Fig. 5.4 shows a typical in situ transmission curve (from the thinnest sample in the range) as a function of chemical-etching time. The three symbols, square, circle, and triangle, show the time during the chemical etching at which optical measurements are performed on the dry sample. The three sets in the total transmission measurements in Fig. 5.4p, and in the EBS measurements in Fig. 5.5 correspond to each chemical etching step, with according symbol. The transmission in Fig. 5.4 decreases to almost a minimum, at half the initial value. A qualitative explanation for such a decrease is that the transport mean free path of the sample is also decreased by a factor 2 . The in situ transmission depends on the transport mean free path of the infiltrated sample, and on the extrapolation ratio at the interface between water and the infiltrated sample. A variation in this in situ transmission is therefore only a possible indication that the mean free path in the dry sample is changed.

In Fig. 5.4p, the total transmission measurements of the samples versus the thickness and the etching time are plotted. According to Eq. 2.62, the inverse total transmission is linear in the thickness $L_{\mathrm{e}} \equiv L+2 \tau_{\mathrm{e}} \ell$ in the absence of absorption or localization. Either 

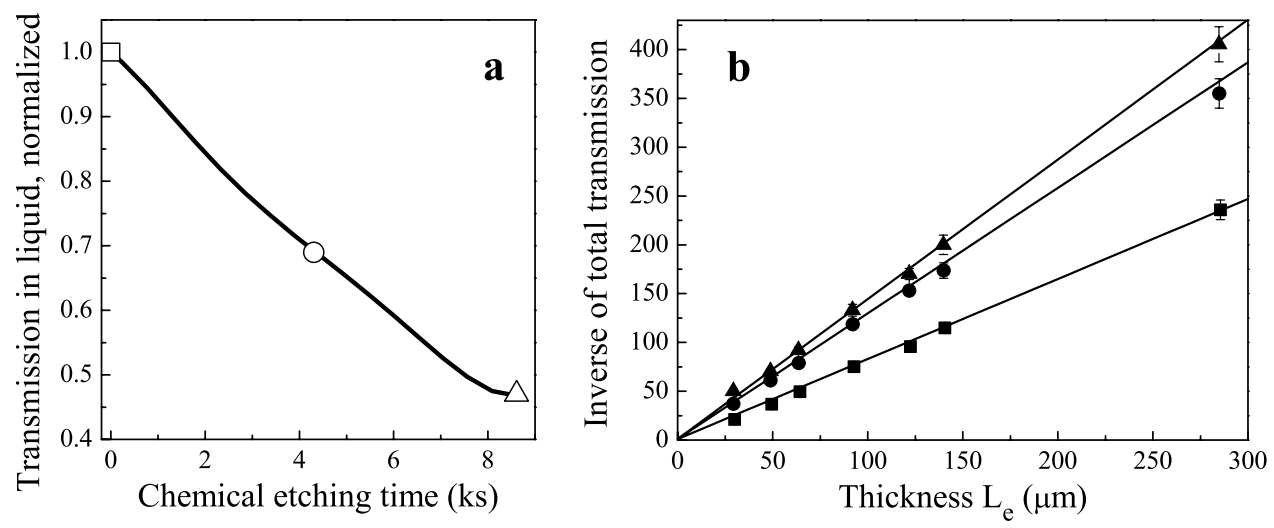

Figure 5.4: Total transmission through a porous GaP sample $\left(N=5 \times 10^{17} \mathrm{~cm}^{-3}\right.$, etched at $\left.14.5 \mathrm{~V}\right)$ as function of chemical-etching time. In (a), the in situ transmission during chemical etching is shown, normalized to the value before etching (square). The chemical etching is stopped twice after $4300 \mathrm{~s}$ (at the circle and the triangle). A range of samples with different thicknesses is prepared in the same way. In (b), the inverse of the total transmission, after drying the samples, is plotted against the thickness, and the chemical etching time: before etching (squares), after one (circles) and two (triangles) chemical-etching steps. The full curves are fits to the diffusion theory, without absorption or localization.

absorption or localization induces an exponential decrease of the total transmission with thickness. The three sets of measurements in Fig. 5.4p are fitted to Eq. 2.62, to obtain the transport mean free path. The extrapolation ratio $\tau_{\mathrm{e}}$, necessary to analyze both total transmission and EBS measurements, is determined by an escape function measurement. The experimental values of the extrapolation ratio, the effective refractive index and the transport mean free path determined by the total transmission are summarized in table 5.1 . As can be seen from the total transmission measurements (Fig. 5.4p and its summary in table 5.1), the transport mean free path of the dry samples hardly reflect the change in transmission of the infiltrated samples. The total transmission stays linear in thickness even for very low transport mean free path, after chemical etching (where $k \ell \simeq 3.4$ ).

\begin{tabular}{|l||c|c|c|c|c|}
\hline $\begin{array}{l}\text { chemical etching } \\
\text { time }\end{array}$ & $n_{\mathrm{e}}$ & $\tau_{\mathrm{e}}$ & $\begin{array}{c}\text { trans. } \\
\text { in liquid }\end{array}$ & $\begin{array}{c}\ell \text { from TT } \\
(\mu \mathrm{m})\end{array}$ & $\begin{array}{c}\ell \text { from EBS } \\
(\mu \mathrm{m})\end{array}$ \\
\hline \hline before, $t=0 \mathrm{~s}$ & $1.42 \pm .05$ & $2.05 \pm .22$ & $\equiv 1$ & $0.40 \pm .02$ & $0.37 \pm .03$ \\
middle, $t=4300 \mathrm{~s}$ & $1.31 \pm .05$ & $1.60 \pm .20$ & 0.69 & $0.30 \pm .02$ & $0.27 \pm .02$ \\
end, $t=8600 \mathrm{~s}$ & $1.29 \pm .05$ & $1.52 \pm .19$ & 0.47 & $0.28 \pm .02$ & $0.26 \pm .02$ \\
\hline
\end{tabular}

Table 5.1: Optical properties of a porous sample as a function of chemical-etching time. The effective refractive index $n_{\mathrm{e}}$, and therefore the extrapolation ratio $\tau_{\mathrm{e}}$, are determined by an escape function measurement. The transmission in liquid is the in situ transmission during chemical etching and is taken from Fig. 5.4a. The transport mean free path $\ell$ is determined both from the total transmission measurements (TT, see Fig. 5.4p) and EBS (see Fig. 5.5 a). 

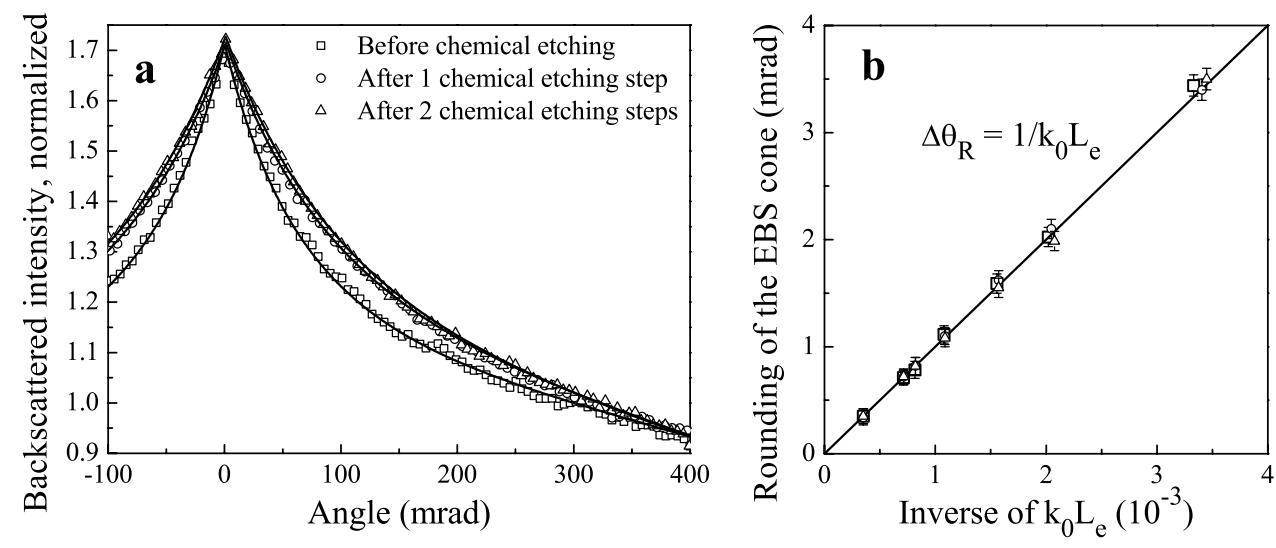

Figure 5.5: EBS measurements of porous GaP samples as a function of chemical etching: before (squares), after one (circles) and two (triangles) etching steps (see Fig. 5.4). In (a), the EBS cones of a single sample becomes wider with etching time, the transport mean free path decreases. In $(b)$, the rounding of the top of the EBS cone is plotted against the inverse of the thickness. Without absorption or localization, the EBS cone rounding is proportional to the inverse of the thickness. Chemical etching reduces the transport mean free path, without introducing any absorption or localization effect.

In the first study of the scattering strength of porous $\mathrm{GaP}[82,83]$, the total transmission of samples with $k \ell \simeq 3$ was also linear with thickness. At the same time, the rounding of the EBS cone was shown to exceed the finite thickness effect, and to be only explainable by the onset of Anderson localization. The subtle localization effect is tested, on the chemicallyetched samples presented here, with the sensitive EBS measurement.

The EBS measurements of the porous sample (from Fig. 5.4 as anction of chemicaletching time are plotted in Fig. 5.5 a. The EBS cone widens with chemical-etching time. The EBS cones for the two chemically-etched samples lie almost on top of each other, indicating a minute variation of the transport mean free path during the second chemicaletching step. Each EBS cone is fitted to Eq.2.81 in order to evaluate the mean free path. The values of the mean free path determined by EBS are summarized in table 5.1 The two determinations of the transport mean free path, by total transmission and EBS, agree with each other, within experimental accuracy, in opposition to the discrepancy found in reference [81] for comparable samples.

The top of the EBS cone of the chemically-etched samples is also investigated, and its rounding is plotted as function of thickness in Fig. 5.5p. The EBS rounding in the case of all the samples lies on the line $\Delta \theta_{\mathrm{R}}=1 /\left(k_{0} L_{\mathrm{e}}\right)$, characteristic of the finite thickness effect, without absorption or localization. No effect of localization is seen in our samples close to the Ioffe-Regel criterion, $k \ell_{\mathrm{B}} \simeq 1$.

The parameter $k \ell$ in the chemically-etched samples presented in this thesis is comparable to the one from samples in both previous studies [81,83]. In reference [83], the EBS rounding of samples with $k \ell \simeq 3$ was attributed to the onset of localization. Our EBS measurements on the very same samples from reference [83] reproduced the measurements of reference [83]. In reference [81], a qualitative discrepancy between the transmission of the 
infiltrated samples and the width of the EBS cone was found. Neither deviation from the diffusion expectations are found in the present study. Localization effects are expected to appear when $k \ell$ approaches 1 , which is the parameter which can be determined experimentally. The real criterion for localization involves the Boltzmann mean free path $\ell_{\mathrm{B}}$, (or the scattering mean free path $\ell_{\mathrm{sc}}$ modified to take anisotropic scattering into account, but not interference) which is particularly difficult to determine in strongly scattering media. The SEM images of samples from the previous studies [81-84] show a more isotropic porous structure than for the samples presented here. Apart from the (small) difference in $k \ell$ between our samples and those in reference [83], the absence of localization effect in our samples may be due to a difference in Boltzmann mean free path.

\subsection{Conclusions}

Electrochemical etching of GaP produces a strongly-scattering porous structure. After electrochemical etching, a highly-reflecting top-layer of bare $\mathrm{GaP}$ modifies the optical properties of the interface of the diffusive material. After removal of the top-layer, the transport mean free path of the material is easily measured by EBS. Samples with very strong scattering were produced by electrochemical etching, up to $k \ell \simeq 3.5$ for samples made from wafers of doping concentration $N=5 \times 10^{17} \mathrm{~cm}^{-3}$. Chemical etching increases the average pore size of porous samples. The scattering strength of porous samples can be increased by chemical etching. The samples prepared according to the anodic, photochemical and chemical processes present all the characteristics of diffusion without absorption or localization, up to very strong scattering. 



\section{Chapter 6}

\section{Anisotropic wave diffusion in porous GaP}

\subsection{Introduction}

Diffusion is a process based on the randomization of the direction of propagation of a particle or wave, on the scale of the transport mean free path $\ell$, inside a material of size much bigger than $\ell$. Intuitively, diffusion is therefore an isotropic phenomenon. In a material with multiple scattering which is statistically invarian 11 by any rotation, symmetry requires isotropy. In a material which is not statistically invariant by any rotation, the properties of light scattering are expected to be dependent on direction, and in particular, the diffusion can be anisotropic. In table 6.1 the differences between the symmetry of the scatterers and the symmetry of the material, (and therefore the symmetry expected for diffusion), are shown. The typical model system for an isotropic medium is a homogeneous distribution of spherical scatterers (see upper-left corner of table 6.1), and usually point scatterers ${ }^{2}$ A spherical scatterer has scattering properties which are independent of the direction of the incident light, but can still depend on the angle between incident and scattered directions. A Mie sphere is by definition a spherical scatterer, as is a point scatterer. Note that the point scatterer has the additional property of isotropic scattering, meaning that after one scattering event, the scattered light is distributed isotropically. A good example of a homogeneous distribution of spherical scatterers is a random aggregation (or glass phase) of silica or titanium dioxide spheres (such as found in toothpaste or white paint). A medium containing isotropic scatterers can still be anisotropic, because of the material between scatterers. If the correlation function of the position of a distribution of isotropic scatterers depends on direction, then the material is anisotropic (see lower-left corner of table 6.1, where the probability to find a scatterer just above or below another scatterer is much higher than

\footnotetext{
${ }^{1} \mathrm{~A}$ random material is not invariant by translation, or rotation, since this invariance requires the exact position and shape of all the scatterers to be symmetrical. Considering the distribution of scatterers instead of the scatterers themselves leads to statistical properties. For example, a material with an homogeneous distribution of isotropic scatterers is statistically invariant by rotation and translation.

${ }^{2}$ Section 2.6 for example, makes explicit use of a homogeneous distribution of point scatterers to calculate the EBS contribution.
} 


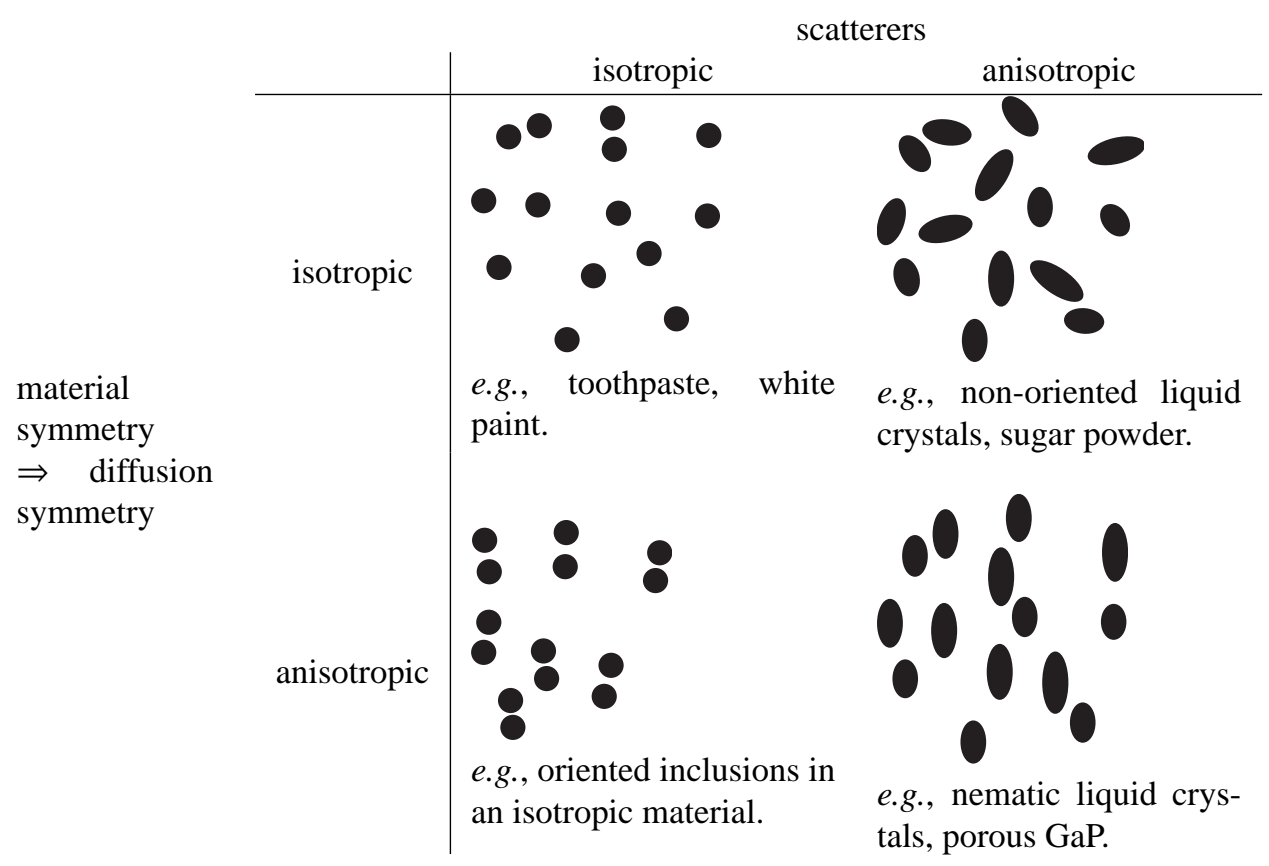

Table 6.1: Summary and examples of isotropic or anisotropic materials, depending on the microscopic properties of the scatterers.

the probability to find one just on the left or right). Isotropic inclusions in a birefringent material also produces an anisotropic material. A non-spherical scatterer (such as the ellipsoids in the right column of table 6.1 has scattering properties depending on its orientation compared to the direction of the incident light. If the distribution of orientations of nonspherical scatterers is isotropic (i.e., all orientations are equiprobable) the material does not have any macroscopic preferential direction (see upper-right corner of table 6.1). Most liquids or colloids (like 'liquid crystals' above the liquid phase transition) which contain non-spherical scatterers are still isotropic because of the randomization of the orientation of individual scatterers. By aligning the non-spherical scatterers with a single macroscopic direction, the material is made anisotropic (see lower-right corner of table 6.1). A transition from isotropic to anisotropic can be found for example in nematic liquid crystals, aligned with an external magnetic field [120]. The porous GaP samples formed by electrochemical etching (see section 3.2 have a well defined preferential direction: pores grow in a direction parallel to the potential gradient and usually normal to the surface of the wafer. Other systems in which diffusion is expected to be anisotropic include biological tissue, nematic liquid crystals or photonic crystals of non-cubic symmetry [33].

The subject of an angular-dependent mean free path or diffusion constant is very seldom considered. Interest in the propagation of light in nematic liquid crystals has provided the first theoretical [180-185] and experimental [186-190] insights into anisotropic diffusion. The first observations of anisotropic diffusion on a sample in the solid phase were done in porous $\mathrm{GaP}[90,94]$. Both scattering and anisotropy are much stronger in porous $\mathrm{GaP}$ than 
Figure 6.1: Schematics in 2D of the discrete anisotropic hopping model. A particle at position $(i, j, k)$ can hop during $\delta t$ in the direction $\alpha$ with a rate $p_{\alpha}$ and $a$ step length $\ell_{\alpha}$. The third dimension, along $z$, has to be seen orthogonal to $x$ and $y$, with index $k$.

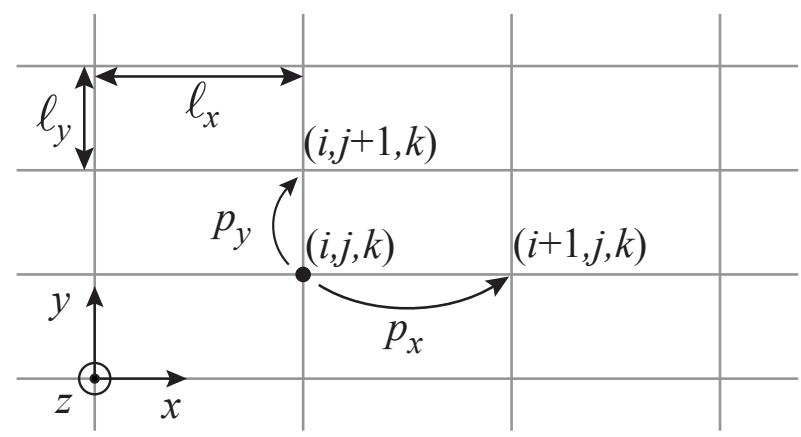

in nematic liquid crystals.

The characteristics of porous GaP, strong scattering $[82,83]$ and strong anisotropy $[90$, 94] raise the possibility to achieve, or tune, Anderson localization thanks to anisotropy. It is well known [23] that Anderson localization is always present in $1 \mathrm{D}$ and 2D systems. It is believed $[88,89]$ that decreasing the dimensionality from $3 \mathrm{D}$ to $1 \mathrm{D}$ in a continuous way (i.e., by increasing anisotropy) reduces the requirements to reach Anderson localization. An anisotropic 3D system lies in this dimensionality description somewhere between 3D and 2D and would therefore be a good candidate for (anisotropic) Anderson localization.

In the following section, a generalization of diffusion to an anisotropic medium is made. In sections 6.3 and 6.4 , stationary and dynamic diffusion experiments on porous $\mathrm{GaP}$ are presented. In section 6.5 an anisotropic wave diffusion experiment on porous $\mathrm{GaP}$ is presented, namely the enhanced backscattering.

\subsection{Generalizing diffusion to an anisotropic medium}

Theoretical work on anisotropic diffusion has been done [180-185], but up till now mainly focusses on the origin of the scattering in nematic liquid crystal: the fluctuations of the nematic director. A microscopic derivation, similar to the derivation of the Boltzmann equation of section 2.4 for an anisotropic medium has not yet been made. Without a microscopic derivation leading to an anisotropic diffusion equation, a phenomenological input for the anisotropic diffusion model is necessary.

\subsubsection{Anisotropic hopping model}

A possible realization of an underlying model describing 3D anisotropic diffusion is a $3 \mathrm{D}$ hopping model. A particle sits on a 3D grid, at position $(i, j, k)$, represented in Fig. 6.1. During the time $\delta t$ the particle has a probability to hop on an adjacent point on the grid. The rates at which the particle is hopping along the $x, y$ and $z$ axes are respectively $p_{x}$, $p_{y}$ and $p_{z}$ and the step sizes are respectively $\ell_{x}, \ell_{y}$ and $\ell_{z}$. The rates of hopping in a certain direction do not depend on where the particle was coming from. In the case of an isotropic hopping model $\left(p_{x}=p_{y}=p_{z}\right.$ and $\ell_{x}=\ell_{y}=\ell_{z}$ ) such angle-independent rates are equivalent to isotropic scattering. The step size, in the isotropic case, can likewise be seen as the (transport) mean free path of the particle in the medium. If the rates $p_{\alpha}$ and step 
sizes $\ell_{\alpha}$ are anisotropic, both dynamic and stationary anisotropies are introduced. Such a particle hopping on a grid performs a random walk. The concentration of a collection of non-interacting particles hopping on a grid follows a diffusion equation. Indeed, the time evolution of the concentration $C_{i, j, k}(t)$ of particles at position $(i, j, k)$ on the grid and at time $t$ obeys the relation

$$
\begin{aligned}
\frac{\mathrm{d}}{\mathrm{d} t} C_{i, j, k}(t)= & p_{x}\left[C_{i+1, j, k}(t)-2 C_{i, j, k}(t)+C_{i-1, j, k}(t)\right] \\
+ & p_{y}\left[C_{i, j+1, k}(t)-2 C_{i, j, k}(t)+C_{i, j-1, k}(t)\right] \\
+ & p_{z}\left[C_{i, j, k+1}(t)-2 C_{i, j, k}(t)+C_{i, j, k-1}(t)\right] .
\end{aligned}
$$

The concentration at $(i, j, k)$ can vary, for each axis, because of hopping out in either direction, or hopping in from either direction. In a continuous-space description, $C_{i, j, k}(t) \rightarrow$ $C\left(i \ell_{x}, j \ell_{y}, k \ell_{z} ; t\right)$. In the limi $\ell_{x, y, z} \rightarrow 0$, the right-hand side of Eq. 6.1 is the sum of the second derivatives with respect to each axis. Therefore

$$
\begin{aligned}
& {\left[\frac{\partial}{\partial t}-\mathbf{D}: \nabla^{2}\right] C(x, y, z ; t)=0,} \\
& \text { where }\left\{\begin{aligned}
D_{\alpha \alpha} & =p_{\alpha} \ell_{\alpha}^{2} \\
D_{\alpha \neq \beta} & =0 .
\end{aligned}\right.
\end{aligned}
$$

Following a very simple continuous-space hopping model, an anisotropic diffusion equation is retrieved. The diffusion tensor defined here is anisotropic. Both stationary $\left(\ell_{\alpha}\right)$ and dynamic $\left(p_{\alpha}\right)$ parameters of the model influence the diffusion tensor. From this hopping model, the mean free path $\ell_{\alpha}$ and the diffusion tensor $\mathbf{D}$ can be independently set as isotropic or anisotropic. By setting the mean free path isotropic, $\ell_{\alpha}=\ell$, an anisotropic diffusion tensor is obtained when the rates $p_{\alpha}$ are not all equal.

\subsubsection{Diffusion with anisotropic diffusion tensor}

The anisotropic diffusion equation found in previous section, Eq. 6.2, is a very intuitive generalization of the diffusion equation (Eq. 2.53 to anisotropic materials. The addition of absorption into the hopping model is instantaneous, by specifying the rate $1 / \tau_{\mathrm{a}}$ at which $C_{i, j, k}(t)$ decays because of absorption.

The generalization of the diffusion equation to an anisotropic medium in the cartesian basis of coordinates $x_{i}$ is therefore

$$
\left[\frac{\partial}{\partial t}-D_{i j} \nabla_{x_{i}} \nabla_{x_{j}}+\frac{1}{\tau_{\mathrm{a}}}\right] W_{\omega}^{\mathrm{rad}}(\mathbf{r}, t)=0 .
$$

In the basis of the hopping-model grid, $D_{i j}$ is diagona ${ }^{4}$. Note that in the basis of coordinates $X_{i}$ such that

$$
x_{i} \equiv X_{i} \sqrt{D_{i i} / D_{z z}}
$$

\footnotetext{
${ }^{3}$ The step sizes $\ell_{x, y, z}$ do not need to vanish, but be much smaller than the typical length scale of diffusion. The diffusion regime only sets in when the size of the sample is much bigger than the transport mean free path.

${ }^{4}$ For samples presented in this thesis, the diffusion tensor $D_{i j}$ is diagonal in the natural basis for a slab, as is shown in section 6.3
} 


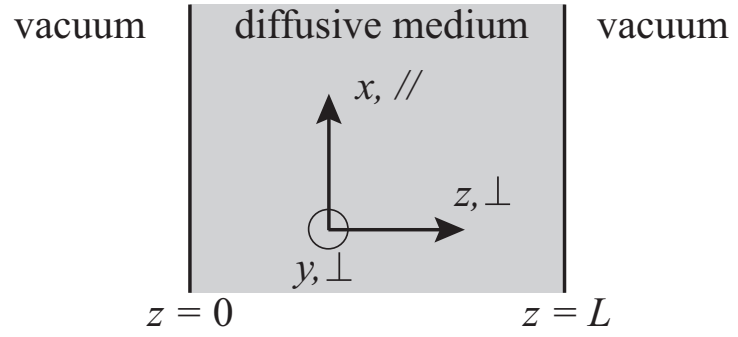

the diffusion equation is isotropic,

$$
\left[\frac{\partial}{\partial t}-D_{z z} \nabla_{X}^{2}+\frac{1}{\tau_{\mathrm{a}}}\right] W_{\omega}^{\mathrm{rad}}(\mathbf{r}, t)=0
$$

where the diffusion constant $D_{z z}$ has been chosen for consistency with the derivation for the diffuse transmission (Eq. 6.8.

The identification of the basis where the diffusion equation for an anisotropic material is isotropic allows in principle the use of the isotropic results found in sections 2.5 and 2.6 together with the scaling of Eq. 6.4 to obtain the anisotropic diffusion results. This scaling method is used in the present section to give predictions for the experiments shown in sections 6.3 to 6.5 Nonetheless, the theoretical results considering only the anisotropic diffusion tensor are not fully valid. The derivations in sections 2.5 and 2.6 show the importance of the boundary conditions and the source term in a diffusion problem. All stationary diffusion results (see Eqs. 2.62 and 2.81 in particular) depend on the transport mean free path $\ell$ and not on the diffusion constant $D$. Stationary measurements in the anisotropic case are therefore expected to depend on the anisotropic mean free path and not on an anisotropic diffusion tensor. Unfortunately, no generalization of the transport mean free path to an anisotropic medium is available at the moment. Or equivalently, the proper derivation of anisotropic diffusion in bounded media has yet to be done.

\subsubsection{Space-resolved transmission}

The most easy and appealing experiment to show anisotropic diffusion of light is the spaceresolved transmission. In Fig. 6.2 the geometry of the statistically anisotropic material is shown. A slab of material, finite in the $z$ direction and infinite in the $x$ and $y$ directions, is considered. The anisotropic material is taken as uniaxial and its principal axis ${ }^{5}$ serves as definition of the $x$ axis of the basis. All directions perpendicular to the principal axis (e.g., $y$ and $z$ ) are equivalent for the anisotropic material itself, but the finite thickness distinguishes the $z$ axis from the $y$ axis. An incident laser beam is focussed on the front surface of the sample, at $z=0$. The source for diffuse light is taken as a Dirac delta source $S_{0} \delta(x) \delta(y) \delta(z-\ell) \delta(t)$. This approximation of the source as a product of delta functions is valid if the time and space characteristics of the incident pulse are much smaller than the corresponding diffusion characteristics (more particularly the diffuse time $L^{2} / D$ and

\footnotetext{
${ }^{5}$ The denomination $\|$ will also be used for the principal axis. In porous GaP samples, the $\|$ direction is parallel to the direction of growth of the pores. The two, equivalent, directions perpendicular to $\|$ are called $\perp$.
} 
the thickness $L$, respectively). In the scaled basis $X_{i}$, the solution to the diffusion equation is proportional to the time-dependent diffusion Green function found in Eq. 2.55 In the dynamic case, the Dirichlet boundary conditions (in Eq. 4.10) have to be taken. In order for the diffuse intensity to vanish at a distance $z_{\mathrm{e}}$ outside the interface, the source term is anti-symmetrized at $z=-z_{\mathrm{e}}$, and at $z=L+z_{\mathrm{e}}$. This double mirroring gives rise to an infinite sum of exponential terms

$$
\begin{aligned}
& W^{\mathrm{rad}}(\mathbf{r}, t)= \frac{S_{0} D_{z z}}{(4 \pi t)^{3 / 2} \sqrt{D_{x x} D_{y y} D_{z z}}} \exp \left(-\frac{x^{2}}{4 D_{x x} t}-\frac{y^{2}}{4 D_{y y} t}\right) \exp \left(-\frac{t}{\tau_{\mathrm{a}}}\right) \Theta(t) \\
& \times \sum_{m=-\infty}^{\infty}\left[\exp \left(-\frac{[z-A(m)]^{2}}{4 D_{z z} t}\right)-\exp \left(-\frac{[z-B(m)]^{2}}{4 D_{z z} t}\right)\right], \\
& \text { where }\left\{\begin{array}{l}
A(m) \equiv 2 m\left(L+2 z_{\mathrm{e}}\right)+\ell \\
B(m) \equiv 2 m\left(L+2 z_{\mathrm{e}}\right)-2 z_{\mathrm{e}}-\ell .
\end{array}\right.
\end{aligned}
$$

The space-resolved diffuse transmission is the flux of diffuse energy at the back interface $[189,191]$

$$
\begin{aligned}
& T_{\mathrm{d}}(x, y, t) \equiv-\left.D_{z z} \frac{\partial W^{\mathrm{rad}}(\mathbf{r}, t)}{\partial z}\right|_{z=L} \\
& T_{\mathrm{d}}(x, y, t)= \frac{S_{0} D_{z z}}{16 \pi^{3 / 2} \sqrt{D_{x x} D_{y y} D_{z z} t^{5}}} \exp \left(-\frac{x^{2}}{4 D_{x x} t}-\frac{y^{2}}{4 D_{y y} t}\right) \exp \left(-\frac{t}{\tau_{\mathrm{a}}}\right) \Theta(t) \\
& \times \sum_{m=-\infty}^{\infty}\left[A^{\prime}(m) \exp \left(-\frac{A^{\prime}(m)^{2}}{4 D_{z z} t}\right)-B^{\prime}(m) \exp \left(-\frac{B^{\prime}(m)^{2}}{4 D_{z z} t}\right)\right], \\
& \text { where }\left\{\begin{array}{l}
A^{\prime}(m) \equiv(2 m+1)\left(L+2 z_{\mathrm{e}}\right)-\ell-2 z_{\mathrm{e}} \\
B^{\prime}(m) \equiv(2 m+1)\left(L+2 z_{\mathrm{e}}\right)+\ell .
\end{array}\right.
\end{aligned}
$$

Eq. 6.8 depends on time, and on the displacement $x$ and $y$ from the origin. At $x=y=0$, i.e., at the position on the back surface just opposite to the point source, the time evolution of the transmitted light only depends on $D_{z z}$. The time evolution of light transmitted at $y=0$ (resp. $x=0$ ) depends only on the two components $D_{z z}$ and $D_{x x}$ (resp. $D_{y y}$ ). A useful tool to analyze a time-resolved transmission curve is to look at the diffuse traversal time $t_{\text {diff }}$,

$$
t_{\mathrm{diff}}(x, y) \equiv \frac{\int t T_{\mathrm{d}}(x, y, t) \mathrm{d} t}{\int T_{\mathrm{d}}(x, y, t) \mathrm{d} t} .
$$

Qualitatively, $t_{\text {diff }}$ can be derived in an anisotropic material without absorption as follows. The time to diffuse through a material of thickness $L$ and diffusion constant $D$ scales with $L^{2} / D$. In order to cross the slab at an angle $\theta$ from the normal to the interface, light has to travel a distance $L(\theta)=L / \cos \theta$. Along this direction $\hat{\mathbf{u}}(\theta)$, light diffuses with a diffusion constant $D(\theta) \equiv \hat{u}_{i} D_{i j} \hat{u}_{j}$. The traversal time across the slab at an angle $\theta$ in the $(x z)$ plane scales with

$$
t_{\text {diff }} \propto \frac{L^{2}(\theta)}{D(\theta)}=\frac{L^{2}}{D_{z z}} \frac{\left[1+(x / L)^{2}\right]^{2}}{1+(x / L)^{2}\left(D_{x x} / D_{z z}\right)},
$$




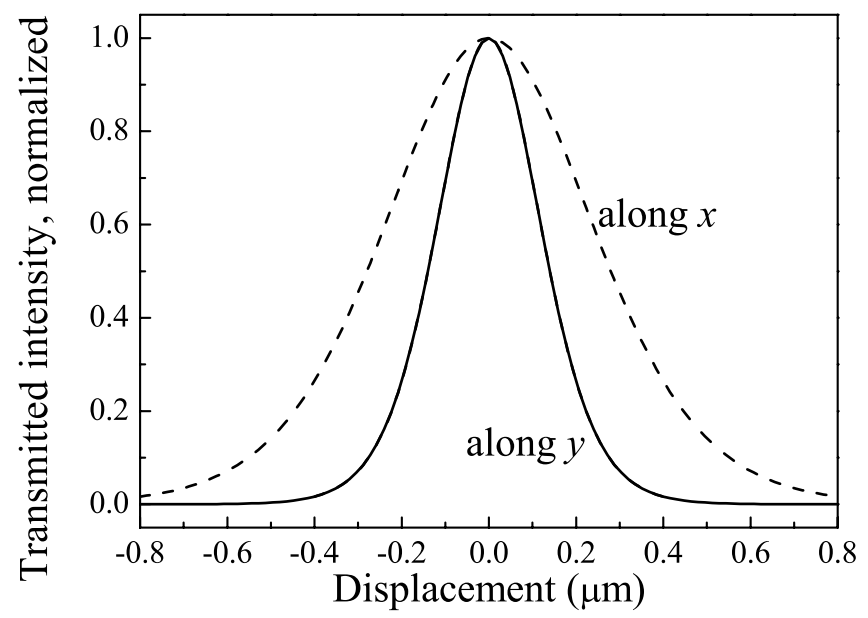

Figure 6.3: Cross-sections along $x$ (dashed curve) and $y$ (full curve) of the spatial distribution of the stationary diffuse transmission. The parameters are chosen from the experiment in sections 6.3 through 6.5. in particular $\sqrt{D_{x x} / D_{y y}}=4$. The cross-section along $x$ with the axis scaled by a factor $1 / \sqrt{4}$ is indistinguishable from the cross-section along $y$.

where $x=L \tan \theta$ is the displacement on the back interface due to crossing the slab at an angle $\theta$. The same equation (Eq. 6.11) holds for the traversal time for a displacement $y$ if the angle $\theta$ is in the (yz) plane, and for $D_{x x} \rightarrow D_{y y}$. The role of absorption is to reduce the diffuse transmission after the absorption time. The traversal time is therefore slightly decreased when absorption is present.

From the exact solution (Eqs. 6.8 and 6.10) and the qualitative derivation (Eq.6.11), the diffuse transversal time is found to be roughly proportional to the square of the displacement from the origin. Obviously, whatever the values of the components of the diffusion tensor, light will be transmitted more quickly for $x=y=0$ since there is no additional transport along the $x$ and $y$ axes.

By integrating Eq. 6.8 over time, a stationary solution to the space-resolved transmission is found. The spatial profile of the diffuse transmission is plotted in Fig. 6.3, with typical parameters $\left(D_{x x}=4 D_{y y}, \ell, \tau_{\mathrm{a}}\right)$ from the experiments in following sections. Scaling the $x$ axis by a factor $1 / \sqrt{4}$ makes the cross-sections along $x$ and $y$ indistinguishable. The ratio of the widths of the two distributions, along $x$ and $y$, is found to be

$$
\frac{\Delta x_{\text {stat }}}{\Delta y_{\text {stat }}}=\sqrt{\frac{D_{x x}}{D_{y y}}},
$$

as was expected from the scaling of the anisotropic diffusion equation (Eq. 6.4.

\subsubsection{Enhanced backscattering}

The generalization of EBS to anisotropic diffusion follows the same easy steps as in the previous subsection, but also keeps the same pitfalls. In section 2.6, the EBS cone is derived 


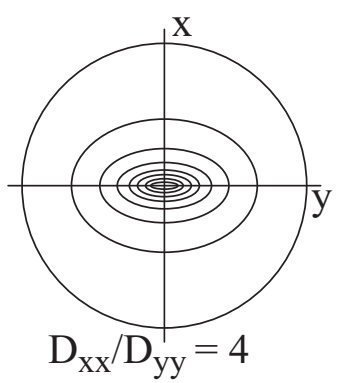

Figure 6.4: Isocontours of an anisotropic EBS cone, for $k_{0} \ell=10, D_{z z}=D_{y y}=D_{x x} / 4, \tau_{\mathrm{e}}=2 / 3$. The distance to the center is $\theta$. The closer the curve is to the center, the higher the backscattered intensity is. The ratio of EBS widths along $x$ and $y$ axis is equal to the square root of the ratio of the $x x$ and yy diffusion tensor components.

in the case of isotropic diffusion. From the bistatic coefficients in Eqs. 2.80 and 2.81 it is obvious that the most important parameter in the EBS is the transport mean free path of the material. Nonetheless, a material in which the diffusion constant is anisotropic (i.e., the diffusion tensor is not proportional to the identity tensor) and the transport mean free path isotropic is considered for the present derivation as the best model up to now.

The derivation of the EBS in section 2.6 stays the same for anisotropic diffusion up till Eqs. 2.73, 2.74 and 2.75, the bistatic coefficients for, respectively, the single scattering, the ladder contribution, and the most-crossed-diagrams contribution. The bistatic coefficients are still to be integrated over the stationary Green function. In the case of anisotropic diffusion, the dynamic Green function for isotropic diffusion, from Eq. 2.55, in the scaled basis (see Eq. 6.4 is considered. The diffusion Green function is Fourier transformed along the $x$ and $y$ components as

$$
G_{\mathrm{d}}\left(z, \mathbf{q}_{\perp}, t\right)=\sqrt{\frac{D_{z z}}{4 \pi t}} \exp \left(-\frac{z^{2}}{4 D_{z z} t}\right) \exp \left(-q_{x}^{2} D_{x x} t\right) \exp \left(-q_{y}^{2} D_{y y} t\right) \Theta(t) .
$$

Integration over time of the dynamic Green function provides the stationary solution

$$
\begin{aligned}
G_{\mathrm{d}}\left(z, \tilde{\mathbf{q}}_{\perp}\right) & =\frac{\exp \left(-z \tilde{q}_{\perp}\right)}{2 \tilde{q}_{\perp}}, \\
\text { where } \tilde{q}_{\perp}^{2} & \equiv \frac{D_{x x}}{D_{z z}} \tilde{q}_{x}^{2}+\frac{D_{y y}}{D_{z z}} \tilde{q}_{y}^{2} .
\end{aligned}
$$

This stationary anisotropic Green function is recognized as the stationary Green function (Eq. 2.56) apart from the renormalized transverse Fourier vector $\tilde{\mathbf{q}}_{\perp}$. The Green function for the semi-infinite medium is derived from the infinite case by using the mirror method (see Eq. 2.78), where internal reflection is taken into account. Note that the mirror method is dependent on the mean free path, and its use therefore requires an isotropic mean free path, assumed also in this section. The integrals in the bistatic coefficients (Eqs. 2.74 and 2.75) can now be performed with the anisotropic stationary Green function in Eq. 6.14. Apart from the renormalized Fourier vector $\tilde{\mathbf{q}}_{\perp}$, the integrals of the bistatic coefficients are performed in the exact same manner than in the isotropic case. 


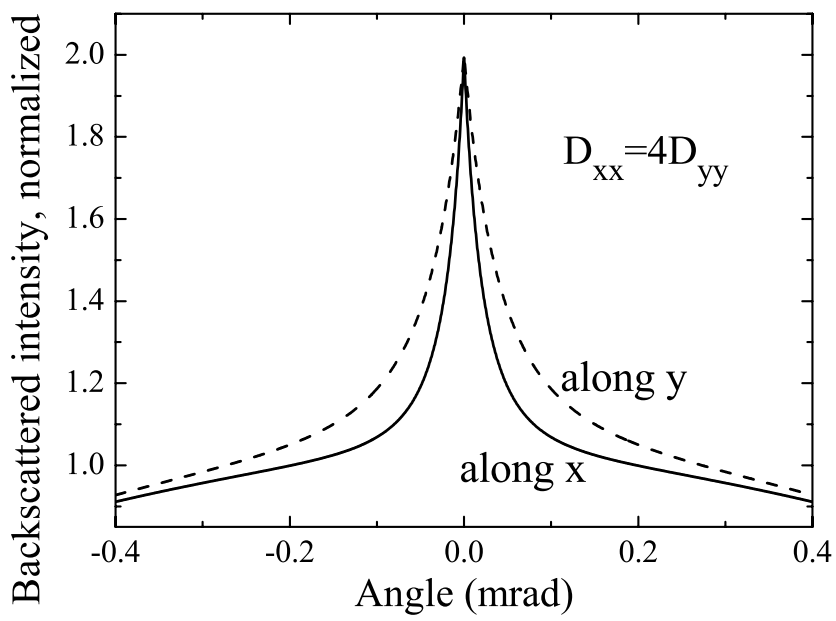

Figure 6.5: Cross-sections of the EBS from an anisotropic material, $k_{0} \ell=10, D_{z z}=D_{y y}=D_{x x} / 4$, $\tau_{\mathrm{e}}=2 / 3$. The cross-section in the $x$ direction (full curve) depends on $D_{x x}$ whereas the cross-section in the $y$ direction (dashed curve) depends on $D_{y y}$. The EBS lineshape of an isotropic sample with $k_{0} \ell=20$ and $\tau=2 / 3$ is here indistinguishable from the $x$ cross-section of the anisotropic EBS cone.

The anisotropic EBS is found as

$$
\begin{aligned}
\gamma_{\ell}\left(\mu_{\mathrm{i}}, \mu_{\mathrm{s}}\right) & =3 \mu_{\mathrm{s}}\left(\tau_{\mathrm{e}}+\frac{\mu_{\mathrm{s}} \mu_{\mathrm{i}}}{\mu_{\mathrm{s}}+\mu_{\mathrm{i}}}\right) \\
\gamma_{\mathrm{c}}\left(\mu_{\mathrm{i}}, \mu_{\mathrm{s}}, \tilde{\alpha}\right) & =\frac{3}{2 \mu_{\mathrm{i}} v} \frac{1}{(\tilde{\alpha}+v)^{2}+u^{2}}\left(1+\frac{2 v \tau_{\mathrm{e}}}{1+\tau_{\mathrm{e}} \tilde{\alpha}}\right), \\
\text { where } u & \equiv k_{0} \ell\left(\mu_{\mathrm{i}}-\mu_{\mathrm{s}}\right), \quad v \equiv \frac{1}{2}\left(\frac{1}{\mu_{\mathrm{s}}}+\frac{1}{\mu_{\mathrm{i}}}\right), \quad \tilde{\alpha} \equiv \tilde{q}_{\perp} \ell .
\end{aligned}
$$

The equations describing the anisotropic EBS exactly map on the results in the isotropic case (Eqs. 2.80 and 2.81). The renormalization $\alpha \rightarrow \tilde{\alpha}$ makes the EBS cone anisotropic. The contours of an anisotropic EBS cone are plotted in Fig. 6.4 The parameter $\tilde{\alpha}$ now depends on the angle between the ( $\mathbf{k}_{\text {in }}, \mathbf{k}_{\text {out }}$ ) plane and the material axes. If $\mathbf{k}_{\text {in }}, \mathbf{k}_{\text {out }}$ and the direction $x$ (resp. $y$ ) are coplanar, the EBS lineshape depends on the (isotropic) transport mean free path, and on the ratio of diffusion tensor components $D_{x x} / D_{z z}$ (resp. $D_{y y} / D_{z z}$ ). If diffusion is faster for example in the tangential $(x)$ than in the depth direction $\left(D_{x x}>D_{z z}\right)$ the width of the EBS cone is reduced by a factor $\sqrt{D_{x x} / D_{z z}}$. The shape of the isotropic EBS cone, from Eqs. 2.80 and 2.81, stays the same in the anisotropic case, for each orientation of the detection plane separately. In other words, it is possible to describe each cross-section of an anisotropic cone with the isotropic EBS model, which leads to a phenomenological transport mean free path depending on the orientation of the detection plane. The crosssection in the $(x z)$ plane, shown in Fig. 6.5. of the anisotropic EBS cone from Fig. 6.4, is described by a transport mean free path $\ell$ and a diffusion tensor such that $D_{x x}=4 D_{z z}$, but is also very well approximated by an isotropic diffusion constant and a renormalized mean free path $\sqrt{4} \ell$. This phenomenological renormalization of the mean free path to account 
for the diffusion tensor is not exact. Renormalizing the mean free path $\ell$ in Eqs. 6.16, 6.17 and 6.18 leads to modify the definition of $u$. The difference between the strict definition of $u$ and the renormalization appears mainly in the wings of the EBS cone, and as a very minute correction (see Fig. 6.5).

It has been suggested [190] that an anisotropic material can be described by an angulardependent transport mean free path, such that $\ell(\phi)=\ell_{\perp} \sin ^{2} \phi+\ell_{\|} \cos ^{2} \phi$, where $\phi$ is the angle between the principal axis (\|) of the material and the detection plane $\left(\mathbf{k}_{\text {in }}, \mathbf{k}_{\text {out }}\right)$. By assuming that the random walk in the three orthogonal directions $(x, y$ and $z)$ is uncoupled, the isotropic EBS result can be applied independently to each direction, with a different mean free path. The description of an anisotropic mean free path is intuitively appealing. Nevertheless, there exists no theory describing anisotropic boundary conditions, necessary to use an anisotropic mean free path. The difference between the anisotropic diffusion result and the renormalized mean free path (see Fig. 6.5) is very small. Therefore, it is not possible to discriminate between the theory developed in this section and the model of an anisotropic mean free path from reference [190].

The theory of an anisotropic diffusion constant is equivalent to the theory successfully applied to photonic crystals with disorder [44,192,193]. A real photonic crystal always has some disorder [194], leading to a transport mean free path typically of the order of $20 \mu \mathrm{m}$ $[44,194]$. The Bragg attenuation length in photonic crystals, depending on the ordering of the structure, reduces the depth in which light within the stop gap can propagate. The difference between the Bragg attenuation length, observed in the depth direction, and the mean free path makes the material effectively anisotropic. The theories presented in these studies $[44,192,193]$ of disordered photonic crystals deal with an anisotropic characteristic length (mean free path and Bragg attenuation length) but also fail to address rigourously the corresponding anisotropic boundary conditions. Therefore the theories of references $[44,192,193]$ can only be seen as (good) approximations of the EBS in a material with a mean free path and a Bragg attenuation length.

\subsection{Stationary anisotropic diffusion}

The anisotropic material used in this chapter $[90,94]$ is formed by anodic etching of a wafer of GaP. The etching direction is made parallel to the surface of the GaP wafer. The thickness of the wafer is $310 \mu \mathrm{m}$. The etching process goes on sufficiently long so that possible edge effects are negligible. The samples are then $2 \mathrm{~mm}$ long, much bigger than all other typical length scales. The anisotropic samples are therefore in a slab geometry, bounded in the $z$ direction. The principal axis (i.e., the direction of etching of the pores) of the material defines the $x$ axis (see Fig. 6.2). The distribution of pores in the whole bulk of the sample is found to be homogeneous [90].

The samples in the anisotropic geometry are made by applying a potential of $10.5 \mathrm{~V}$ on one edge of the wafer, and letting the opposite edge be in contact with an, electrically grounded, sulfuric acid solution of concentration $0.5 \mathrm{~mol} / \mathrm{L}$. The remaining four sides are covered by a layer of silicon nitride $(\mathrm{SiN})$ of thickness $200 \mathrm{~nm}$, to prevent unwanted side

\footnotetext{
${ }^{6}$ Reference [190] quotes $\ell(\phi)=\ell_{\perp} \sin \phi+\ell_{\|} \cos \phi$, which does not reduce to an isotropic mean free path in the isotropic case, and is therefore probably a typo.
} 


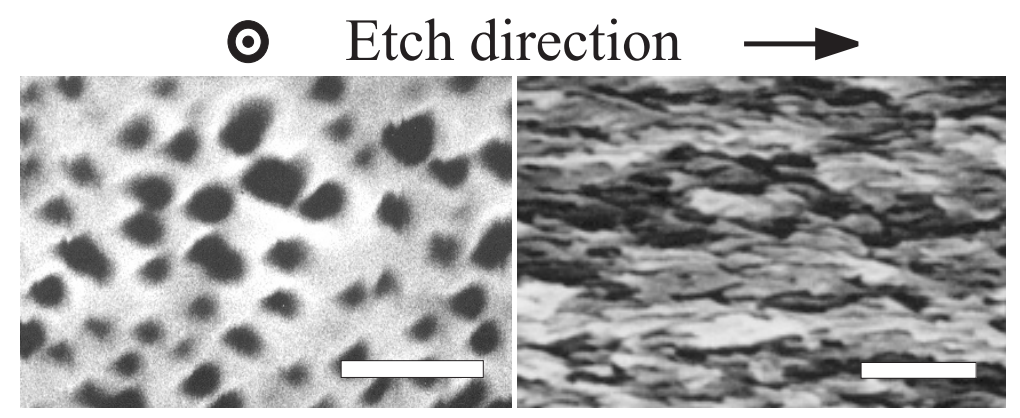

Figure 6.6: SEM images of the anisotropic structure of porous GaP. The left image shows a transversal, and the right image a longitudinal, cross-section of the pores. The material is clearly uniaxial. Scale bars $=1 \mu \mathrm{m}$.

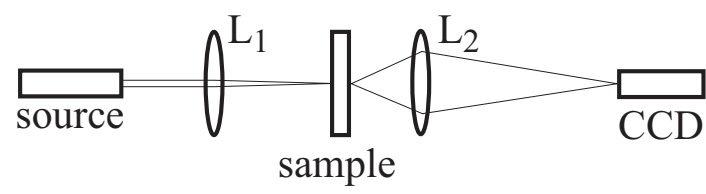

Figure 6.7: Cartoon of the diffuse transmission imaging setup. A HeNe laser beam is focussed through a lens $L_{1}$ on the front side of the sample. The back side is imaged, trough a lens $L_{2}$, on a $C C D$ camera. If the sample is not invariant by rotation around the axis of the laser beam, the image on the CCD is anisotropic.

etching. The obtained samples display a porosity of $46 \%$ and the diameter of the pores is $(70 \pm 30) \mathrm{nm}$. Two scanning electron microscopy (SEM) images, transversal and longitudinal to the pores, of a typical sample are shown in Fig. 6.6 The random distribution of the pores, their diameter close to the wavelength of light and the high refractive index contrast insure that light is strongly scattered. Furthermore, the elongation of the pores, being several times their diameter, induces the anisotropy for light scattering and diffusion.

\subsubsection{Space-resolved transmission}

The most straightforward measurement to show anisotropic diffusion in a slab is letting the light from a pointlike source on one side of the slab diffuse to the other side, spreading unevenly in different directions. The principle of such transmission measurements is described in references $[90,186,188]$, and sketched in Fig. 6.7. A HeNe laser beam $(633 \mathrm{~nm})$ is focussed on the front side of the sample, with a focus width of $10 \mu \mathrm{m}$. The back side of the sample is imaged on a charge-coupled device (CCD) camera. Light propagating through the sample gets diffuse after one transport mean free path, losing memory of its initial polarization along with its initial direction. In transmission through thick turbid material, the incident polarization therefore plays no role.

The image on the CCD camera from an anisotropic sample is shown in Fig. 6.8. The scattered light from a continuous-wave laser through a stationary sample displays a speckle pattern, visible in Fig. 6.8 a. This spatial speckle is described as short-range intensity corre- 
a)

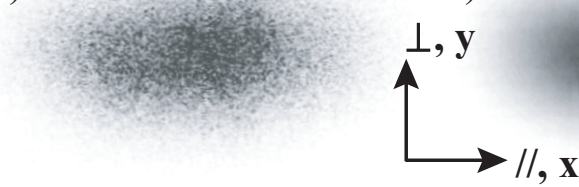

Figure 6.8: Light intensity on the back side of an anisotropic sample, as measured with the CCD camera. Darkest regions have the highest light intensity. (a) Without speckle averaging. (b) With speckle averaging. The diffusion is strongly anisotropic.

lations, called $C_{1}$ in the literature $[12,195]$. The short-range correlation of speckle intensity between two points separated by a distance $\Delta r$ is $[12,196]$

$$
C_{1}(\Delta r)=\frac{\sin ^{2}(k \Delta r)}{(k \Delta r)^{2}} \exp \left(-\frac{\Delta r}{\ell}\right),
$$

where $k$ is the wave vector of the light inside the material, therefore depending on the effective refractive index $n$. The intensity correlation $C_{1}$ depends mainly on the wave vector $k$, and lightly on the mean free path $\ell$ when $k \ell \gg 1$. For all but the most strongly scattering samples, $C_{1}$ only depends on the wavelength of light and the effective refractive index of the material. A study [197] of the near-field spatial speckle pattern of a micro-porous silica glass demonstrated the possibility to determine the effective refractive index of the material. In a material with a broken rotation symmetry, the speckles can be expected to become anisotropid 7 A naïve generalization of the correlation function $C_{1}$ to an anisotropic material suggests the correlation along a certain direction depends on the mean free path and refractive index along this direction. An anisotropic material can display angular-dependence for both mean free path and refractive index (i.e., birefringence). In the present anisotropic samples, diffusion is anisotropic and birefringence can be expected 8 No anisotropy in the spatial intensity correlation function of the porous $\mathrm{GaP}$ samples was observed. An optical microscope is barely able to fully resolve the spatial speckle pattern. A near-field probe, such as used in reference [197], has an uncontrolled anisotropy in the geometry of the tip itself, making the analysis of an anisotropic correlation function very difficult.

By averaging over speckle, i.e., averaging over different speckle patterns formed by different positions but the same orientation of the sample, a smooth diffuse pattern is recovered, as seen in Fig. 6.8 $\mathrm{p}$. The diffuse transmission through the anisotropic sample is clearly anisotropic. Light diffuses farther in the $\|$ direction than in the $\perp$ direction. In the limit of infinitely long and rectilinear pores, i.e., the 2D limit, a wave could propagate along the pores, but be diffuse in the plane orthogonal to the pores. Intuitively, light should propagate more easily along the pores (in the $\|$ direction) than against $(\perp)$. The intensity distribution in Fig. 6.8p is symmetrical around the axes $x$ and $y$. The assumption in section 6.2, that the diffusion tensor of our anisotropic samples is diagonal in the natural basis for the slab and the orientation of the pores, is justified.

\footnotetext{
${ }^{7}$ Although no such theory has been developed yet.

${ }^{8}$ Several experiments suggest birefringence in micro-porous GaP [71] and silicium [198].
} 


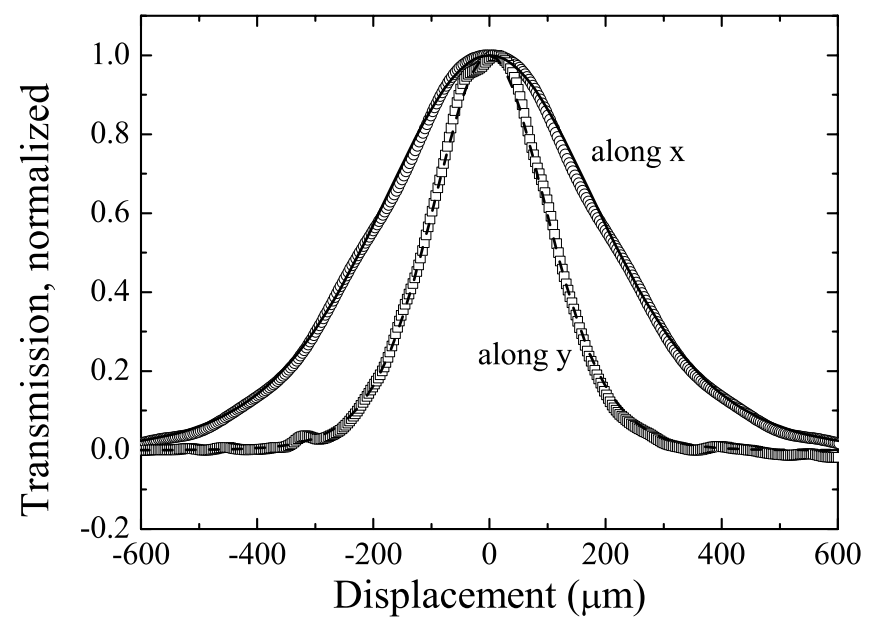

Figure 6.9: Normalized diffuse transmission as a function of displacement at the back side of an anisotropic sample, along the two principal directions, $\|($ or $x)$ in full curve, $\perp$ (or $y)$ in dashed curve. The two curves are fitted to the theory of section 6.2.3. The ratio of widths is $\Delta x_{\text {stat }} / \Delta y_{\text {stat }}=2.1 \pm 0.2$.

Two cross-sections of the image on the CCD (of Fig. 6.8p) are taken, going through the center of the spot, in the $\|$ and $\perp$ directions respectively, as shown in Fig. 6.9. Both cross-sections of the anisotropic transmission spot are fitted to the theory of section 6.2 .3 . Equivalently the $x$ axis can be scaled so that both cross-sections in Fig. 6.9 lie on top of each other. The anisotropy of the transmission spot is $\Delta x_{\text {stat }} / \Delta y_{\text {stat }}=2.1 \pm 0.2$. This ratio of widths should be interpreted, according to the theory of section 6.2.3 as the square root of the ratio of diffusion tensor components $x x$ and $y y$, thus $D_{x x} / D_{y y}=4.4 \pm 0.8$.

The diffusion of light in porous $\mathrm{GaP}$ is much more anisotropic than reported in nematic liquid crystals $[186,188]$ (where the anisotropy in diffusion constant is found around 1.3-1.6). Porous GaP is a strongly anisotropic and strongly scattering material.

\subsubsection{Angular-resolved transmission}

Previous studies of anisotropic diffusion (in particular in the interpretation of the anisotropic EBS cone from nematic liquid crystals [190]) dismissed the problem of the interface properties as non-significant. Section 2.6 shows how important the knowledge of the interface properties is in the analysis of stationary diffusion measurements. In chapter 4 , the interface properties of a diffusive material are found from the angular-resolved transmission (ART), or escape function. There is still no theory of diffusion with anisotropic mean free path. The escape function (see section 4.2.2) in the case of an anisotropic material is also not theoretically known. The ART of the anisotropic material must therefore be determined in order to fully analyze the EBS measurements in section 6.5.1 The ART of the anisotropic material is determined in the same manner ${ }^{9}$ as described in section 4.3 . Of course, the

\footnotetext{
${ }^{9}$ Even in the case of an anisotropic material, the polarization of the incident beam has no effect on the diffuse transmission.
} 


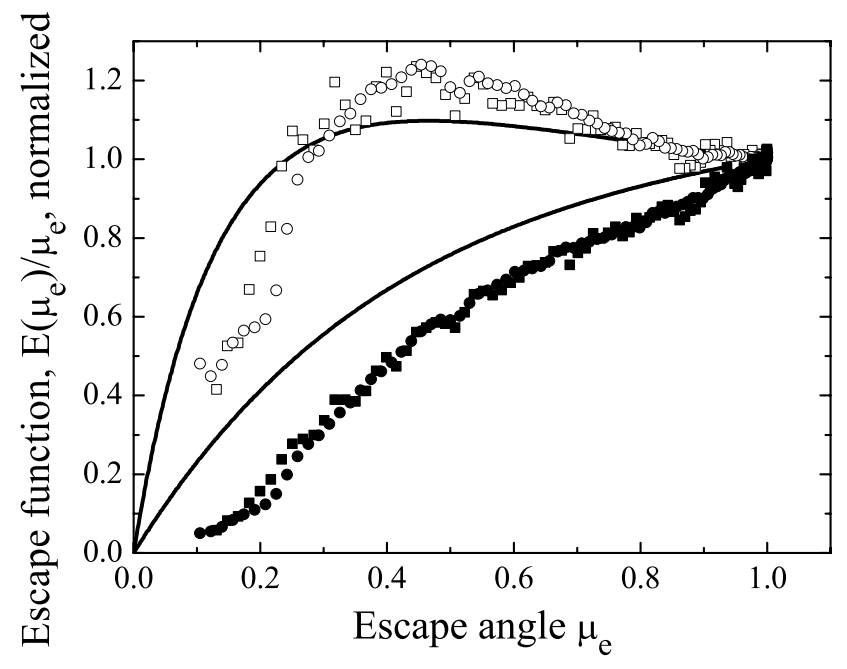

Figure 6.10: Escape function of an anisotropic sample in two orientations, etching direction in (circles) and out (squares) of scanning plane and for two polarizations, $P$ (open symbols) and $S$ (closed symbols). The two full curves are the two polarizations of the closest fit to the theory, with $n$ $=2.45$. The experimental data is independent of direction: the reflectivity properties are isotropic in this sample, but they do not fit with the isotropic theory.

anisotropic material can not be spun around the axis of the incident beam, since it would destroy the potential orientation dependence of the ART. Without spinning the sample, the speckle averaging is much less efficient. The escape function measurements on anisotropic material are shown in Fig. 6.10. The sample can be oriented with the direction of the pores in the plane of detection (\|, circles), or normal to it ( $\perp$, squares). Several measurements for each orientation and polarization ( $\mathrm{P}$ and $\mathrm{S}$ ) are performed, changing the position of the sample each time in order to average over disorder. No difference in angular dependence between the two sets, $\|$ and $\perp$, is found within the experimental uncertainty. The isotropy of the escape function ensures that the interface properties, extrapolation length [128] and reflectivity coefficient $\bar{R}$, are equal in the $\|$ and $\perp$ directions. We estimate the uncertainty in the extrapolation length as $5 \%$. The extrapolation length can not depend on the plane of detection since it is a length in the $z$, or depth, direction. The internal reflection coefficient $\bar{R}$ is derived from the Fresnel coefficients. In a birefringent material for example, such as a nematic liquid crystal, the reflection coefficient can be anisotropic. The EBS width, from Eq. 2.83 , depends on the transport mean free path and the extrapolation length. The extrapolation length being independent of the plane of detection, the ratio of widths of the EBS in the $\|$ and $\perp$ planes are independent of the extrapolation length.

ART measurements are compared to the escape function theory in section 4.2.2. Both polarizations of the best fit to the escape function theory, with a refractive index of 2.45 , are plotted as full curves in Fig. 6.10. Unfortunately, no satisfying quantitative agreement can be found between the measurements of the anisotropic material and the theory within experimental accuracy. The discrepancy can be interpreted as being due to the presence of two extra layers on top of the porous structure. Indeed, the pores can not touch the surface 


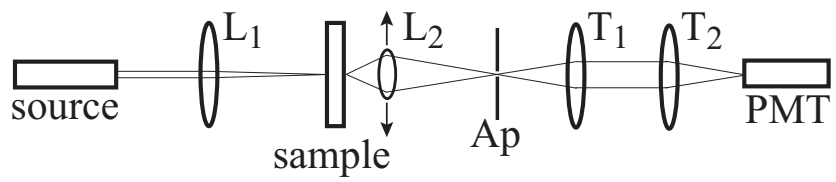

Figure 6.11: Cartoon of the space-and time-resolved diffuse transmission setup. A pulsed source is focussed on the front side of the sample. One point of the back side of the sample is imaged through a lens $L_{2}$, an aperture Ap, and a telescope $T_{1}$ and $T_{2}$, on a fast-response PMT. Moving $L_{2}$ allows different positions on the back side of the sample to be imaged in time.

of the wafer, which leads to a typically $200 \mathrm{~nm}$ thin layer of bare $\mathrm{GaP}$ between the pores and the surface of the wafer. Such a layer is shown in section 4.3.2 to significantly change the ART. The second layer is the remaining SiN layer used to protect the surfaces against etching, which can not be removed without damage to the samples. It is also possible, although unlikely, that an anisotropic mean free path would induce for the escape function a different shape than in the isotropic case, but still independent of the detection plane.

The lack of consistent fit of the ART measurements with the escape function theory prevents the determination of the exact value of the extrapolation length of the anisotropic material.

\subsection{Dynamic anisotropic diffusion}

The space-resolved transmission from section 6.3 can also be performed in a time-resolved way. The setup used to record the time-resolved transmission is sketched in Fig. 6.11 A solid-state pulsed laser $(634 \mathrm{~nm})$, of average power $0.5 \mathrm{~mW}$, of pulse duration $100 \mathrm{ps}$ and repetition rate $20 \mathrm{MHz}$ was used as the source. The laser beam was focussed on the front side of the sample to a $10 \mu \mathrm{m}$ spot. The focus of the laser beam defines the origin of the $x$ and $y$ axes. The back side of the sample was imaged through a lens on an aperture. Displacing the imaging lens in a plane parallel to the surface of the sample allows different successive points of the sample to be imaged on the aperture. The aperture was imaged through a telescope and a monochromator on a photomultiplier tube (PMT). The PMT signal was resolved in time by a time-to-amplitude converter. The origin of time is taken as the arrival time of the incident pulse in the absence of sample. The space- and time-resolved transmission through the anisotropic material is plotted in Fig. 6.12. Three different displacements across the sample along the $y$ axis are shown. Each of the three measurements in Fig. 6.12 shows a peak followed by an exponential decay, characteristic of diffusion (see Eq. 2.66). In the case of the measurements with the displacement along the $y$ direction, all decay times are found to be equal. In the uniaxial porous GaP presented here, the $y y$ and $z z$ components of the diffusion tensor are equivalent, since both are in the direction $\perp$. The measurements of Fig. 6.12 are fitted to the theory of Eq. 6.8, in order to yield the $y y$ and $z z$ components of the diffusion tensor, as well as the absorption time $\tau_{\mathrm{a}}$. A more intuitive way to obtain the diffusion parameters is to consider the diffuse traversal time $t_{\text {diff }}$, from Eq. 6.10. The traversal time increases roughly with the square of the displacement from the origin, as is seen in the inset of Fig. 6.8. The traversal time 


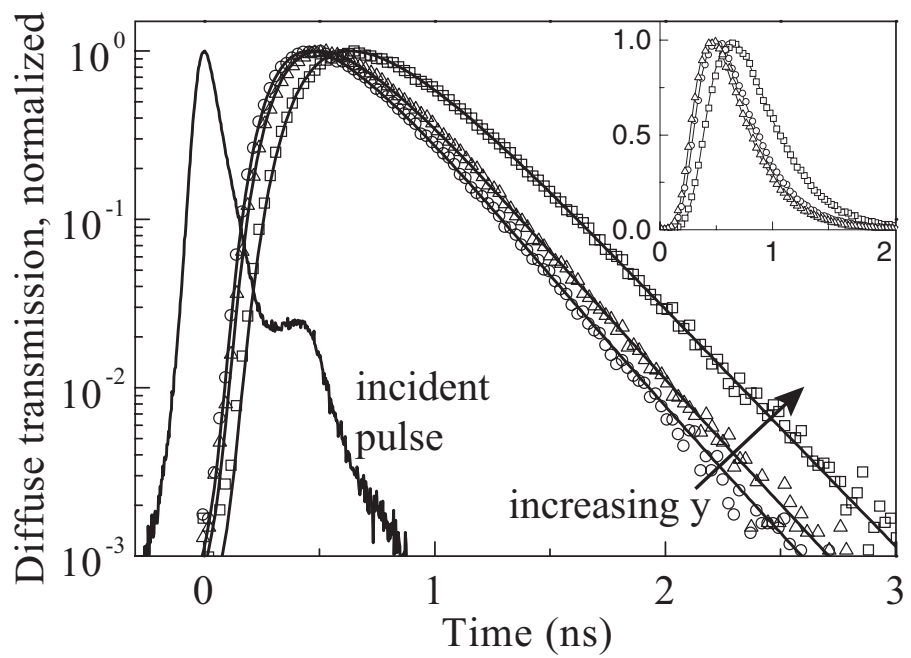

Figure 6.12: Normalized time-resolved transmission through an anisotropic material, for various displacements in the $y$ direction: $y=(0 \pm 10) \mu m$ (circles), $y=(120 \pm 10) \mu m$ (triangles) and $y=$ $(240 \pm 10) \mu m$ (squares). The solid curves are fits to the anisotropic diffusion theory (Eq. 6.8). The solid curve around $t=0$ is the incident pulse. The inset presents the same transmission data in a linear plot. The excellent agreement between the measurements and the theory shows the anisotropic diffusion for light. Courtesy of Patrick Johnson.

for each measurement is plotted in Fig. 6.13, with displacement in the $y$ (triangles) or $x$ (circles) directions. Light traverses the slab earlier through the shortest distance, i.e., for $x=y=0$, where light transport only depends on the $z z$ component of the diffusion tensor. The increase in traversal time depends on the diffusion speed along the two transversal directions and is smaller when the diffusion is faster. From the transmission measurement without $x$ or $y$ displacement, the $D_{z z}$ and $\tau_{\mathrm{a}}$ values can be determined as $D_{z z}=(14.2 \pm$ $0.1) \mathrm{m}^{2} / \mathrm{s}$ and $\tau_{\mathrm{a}}=(0.59 \pm 0.02) \mathrm{ns}$. In a time $\tau$, the average distance the light can propagate with a diffusion constant $D$ is $\sqrt{D \tau}$. The smallest absorption length $L_{\mathrm{a}}$ corresponding to the measured absorption time $\tau_{\mathrm{a}}$ is $L_{\mathrm{a}}=\sqrt{D_{z z} \tau_{\mathrm{a}}}=(91 \pm 2) \mu \mathrm{m}$. The absorption length $L_{\mathrm{a}}$ is here significant since smaller than the thickness of the anisotropic sample $(L=310 \mu \mathrm{m})$. In other porous GaP samples the absorption length has been shown [81,84] to exceed $200 \mu \mathrm{m}$, and in section 5.2.2 to exceed $250 \mu \mathrm{m}$. The samples made in the anisotropic geometry are of good quality although less well controlled than the samples made in the normal geometry (and used in chapters 3, 4 and 5)

The transmission measurements with a displacement in the $x$ (resp. $y$ ) direction also depends on $D_{x x}$ (resp. $D_{y y}$ ) and can be fitted by taking $D_{z z}$ and $\tau_{\mathrm{a}}$ fixed, which yields $D_{v v}=$ $(14 \pm 2) \mathrm{m}^{2} / \mathrm{s}$ and $D_{x x}=(58 \pm 5) \mathrm{m}^{2} / \mathrm{s}$. The fits for the $y$ direction are plotted in Fig. 6.12. The ratio of the components of the diffusion constant is found to be $D_{x x} / D_{y y}=4.1 \pm 0.4$, in agreement with the value found in the stationary transmission measurement. 
Figure 6.13: Diffuse traversal time $t_{\mathrm{diff}}$ as a function of the absolute value of the displacement from the origin in the $x$ (circles) and $y$ (triangles) directions. The full curves are fits to the anisotropic diffusion theory. The dashed curves are plots of the theory with $\pm 10 \%$ variation in the diffusion tensor components. Light is transmitted earlier in the $x$ direction than in the $y$ direction, the diffusion is therefore faster along $x$ than along $y$.

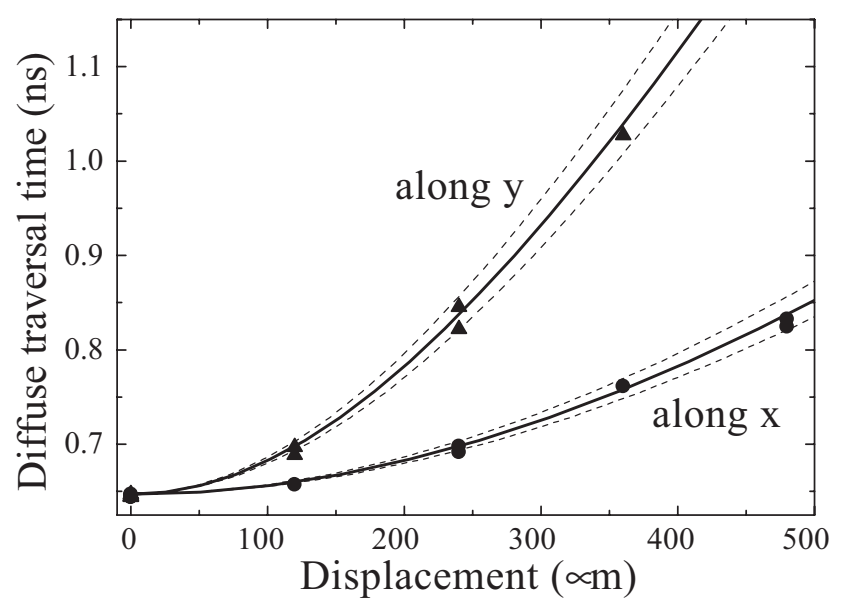

\subsection{Anisotropic wave diffusion}

\subsubsection{Anisotropic enhanced backscattering}

EBS measurements were performed using the off-centered rotation technique [129] and a HeNe $(633 \mathrm{~nm})$ laser as light source. This setup allows to horizontally scan the angle from the exact backscattering direction, with a $900 \mathrm{mrad}$ range and an $0.5 \mathrm{mrad}$ resolution with an illumination area of $2 \mathrm{~mm}$ in diameter. The measured sample can be oriented with etching direction either vertical or horizontal, so that the etching direction is either normal or parallel to the detection plane. In order to average over disorder, a nutation is applied to the sample. The range of the nutation is less than $150 \mathrm{mrad}$. Further averaging was done by performing measurements at four different positions of the samples.

It is possible to measure EBS with various polarizations. Relevant are linear polarization, with parallel or orthogonal analyzer, and circular, with helicity preserving or nonpreserving detection. Using linear polarization, although experimentally easier to perform, is the least preferable solution in combination with an anisotropic material. Indeed, in addition to parallel and cross analyzers, the incident linear polarization can be oriented either parallel or orthogonal to the pores. Furthermore, it has already been noticed [199] that an incident linear polarization in or normal to the scanning plane also introduced an anisotropy in the backscatter cone, due to a trivial polarization effect and not anisotropic diffusion. The authors of reference [199] measured the EBS from concentrated suspensions of polystyrene spheres in the linear (and parallel) polarization channel. The direction of the incident polarization was shown to break the rotational symmetry of the setup and to imply a distinction between two different detection planes, parallel and normal to the incident polarization. The measured backscatter cone displayed different widths along the two planes of detection, parallel and normal to the incident linear polarization. The anisotropy in reference [199] is due to the vector nature of light.

In order to avoid this polarization anisotropy of the backscatter cone, the circular polarization channels can be used. In addition, the helicity preserving channel filters out the 


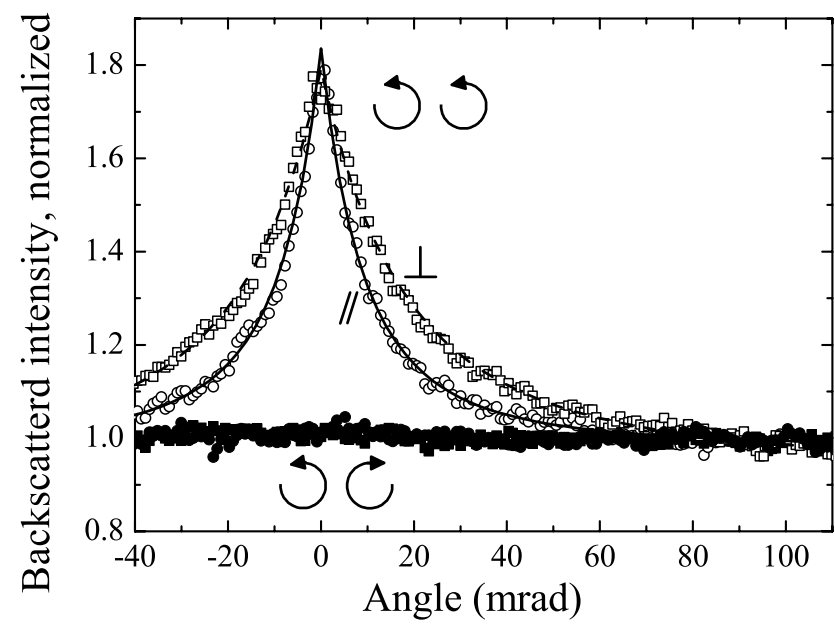

Figure 6.14: Backscattered intensity as a function of angle, normalized to 1 at high angles, of an anisotropic sample in two orientations: etching direction in (\|, circles) and out ( $\perp$, squares) of scanning plane, and two polarizations, helicity preserving (open symbols) and non-preserving (closed symbols). The curves are independent fits to the isotropic EBS theory. The anisotropy is clearly seen in the preserving channel, which implies anisotropic wave diffusion.

single-scattering contribution. This filtering of the single scattering effectively reduces the background of the EBS cone, and ultimately allows to reach the theoretical enhancement factor of 2 [131]. In the present study, the enhancement factor does not reach 2. Paths for light which leave the sample outside the illuminated area but still fall on the detector reduces the EBS enhancement. In the helicity non-preserving channel, only the low orders of scattering remain and contribute to a (much) smaller enhancement factor, as detailed in section 6.5 .2

Fig. 6.14 shows EBS measurements on an anisotropic sample, for the two independent orientations, and for the two circular polarization channels. In the helicity preserving channel (open symbols) the backscatter cone is clearly visible and strongly anisotropic. The narrower EBS cone in the $\|$ direction implies that light diffuses further in the $\|$ than in the $\perp$ direction. In the anisotropic EBS theory (see Eqs. 6.17 and 6.18), the width of the EBS cone depends on the isotropic transport mean free path, the extrapolation length, and the three diagonal components of the diffusion tensor. The ratio of EBS widths along the two directions $x$ and $y$ is the square root of the ratio of diffusion tensor components: $\sqrt{D_{y y} / D_{x x}}=1.90 \pm 0.15$. The anisotropy in the diffusion tensor found in the anisotropic EBS agrees very well with the anisotropy found in the dynamic transmission in section 6.4.

The isotropic transport mean free path from this anisotropic sample can be calculated. The width of the EBS cone in the $\perp$ cross-section (in the (yz) plane), depends on the isotropic mean free path and $\sqrt{D_{y y} / D_{z z}}$. In the case of a uniaxial material oriented along the $x$ direction, such as considered here, the $y y$ and $z z$ components of the diffusion tensor are equal. The isotropic mean free path can be determined directly from the $\perp$ cross-section of the EBS cone, along with an estimation of the extrapolation ratio. In section 4.4 measurements of the refractive index of porous GaP in the 'normal' geometry (i.e., pores growing 
normal to the wafer surface) are shown. The refractive index of the samples etched under the same conditions, apart from the geometry, is taken as characteristic of the bulk of the anisotropic samples used in this chapter, namely $n_{\mathrm{e}}=1.40 \pm 0.05$. An interface between a diffusive material with such refractive index and air would have an extrapolation ratio $\tau_{\mathrm{e}}=1.97 \pm 0.22$. The anisotropic samples have two additional interfaces, coming from the extra GaP layer and the SiN coating, having respectively a refractive index of 3.3 and 2.0. Taking the two extra layers into account, the properties of the whole interface can be calculated (see Eq. 4.15) as $\tau_{\mathrm{e}}=2.95 \pm 0.20$. The transport mean free path, measured from the EBS width and corrected for the extrapolation ratio is calculated from Eq. 2.83 The isotropic transport mean free path in the anisotropic samples can be evaluated as $\ell=(0.80 \pm 0.04) \mu \mathrm{m}$.

\section{Another interpretation of the anisotropic diffusion results}

Within the theory for anisotropic diffusion presented in section 6.2, all anisotropic effects are due to the diffusion tensor and not to an anisotropic mean free path. Intuition and rigorous isotropic theory (e.g., chapter 2) shows that stationary measurements are mainly dependent on the mean free path, and dynamic measurements are mainly dependent on the diffusion constant. A theory of anisotropic diffusion including the rigorous treatment of the interface is needed to resolve this discrepancy.

Considering that stationary measurements depend on the mean free path, and dynamic measurements on the diffusion constant, the results of sections 6.3 and 6.5 .1 should be interpreted fully in terms of transport mean free path. The anisotropy in diffusion has now been found in the diffusion tensor and the mean free path, as $D_{\|} / D_{\perp}=4.1 \pm 0.4$ and $\ell_{\|} / \ell_{\perp}=2.00 \pm 0.15$. In the isotropic case, the diffusion constant and the transport mean free path are related such that $D=v_{\mathrm{E}} \ell / 3$ (see section 2.4.4). If this relation between diffusion constant and mean free path still holds in the anisotropic case within each component of the diffusion tensor, the ratio of energy velocity along the two directions $\|$ and $\perp$ can be deduced, as $2.0 \pm 0.3$. It can also be argued [200] that the diffusion constant is the ratio of an anisotropic stationary part $\ell^{2}$ and an isotropic dynamic part $\tau_{\mathrm{mf}}$. Within the anisotropic hopping model, in Eq. 6.2 the diffusion constant is the product of the square of the mean free path and the rate $p=1 / \tau_{\mathrm{mf}}$. The mean free time, being a probability to scatter after a certain time, can also be in principle anisotropic, since the probability to scatter in a certain time in different directions can be different, as is shown in the hopping model from section 6.2.1. The mean free time in the anisotropic porous $\mathrm{GaP}$ is found to be isotropic within $20 \%$.

\subsubsection{EBS in helicity non-preserving channel}

Before discussing the measurements on EBS in the helicity non-preserving channel (see Fig. 6.14, closed symbols), a short review of the experimental and theoretical literature on the subject is presented.

The EBS cone has been shown to exist in the cross-polarized (linear) channel in the case of Rayleigh scatterers and to slowly vanish with increasing anisotropy of the scatterers $[201,202]$. EBS has also been shown to exist in the helicity non-preserving channel in the case of Rayleigh scatterers and to disappear with optical activity [132]. 
Measurements on nematic liquid crystals [190,203] show the absence of EBS cone, within $1 \%$, in the cross-polarized channel, or an enhancement factor evaluated to a maximum of 3\% [202]. An EBS cone in the linear orthogonal channel has been measured [204] from suspensions of polystyrene spheres of diameter $0.2 \mu \mathrm{m}$ and $1 \mu \mathrm{m}$, with enhancement of respectively $10 \%$ and $20 \%$. EBS cones in the linear orthogonal and in the helicity nonpreserving channels of a cloud of cold rubidium atoms were reported, with enhancements around $10 \%$ [205].

Nematic liquid crystals are very anisotropic scatterers and are birefringent. Both effects contribute to the reduction of the enhancement factor in the cross polarized channel. Spherical colloids are neither very anisotropic nor birefringent, and therefore display an enhancement in the cross-polarized channel. The physics of the EBS cone in cold atoms is beyond the reach of the classical theories mentioned here [132, 201,202].

The EBS measurements of anisotropic porous $\mathrm{GaP}$ in the helicity non-preserving channel, as seen from the filled symbols in Fig. 6.14, do not show any observable EBS cone, for both orientations of the sample. More quantitatively, the enhancement factor in the nonpreserving polarization channel is smaller than $2 \%$. The scattering in porous GaP is very anisotropic, reducing the expected enhancement factor. Another argument for the absence of EBS cone in the non-preserving polarization channel is the polarization dependence of the refractive index of the material (birefringence, or optical activity). In the case of liquid crystals [190,203], the birefringence introduces a large phase shift between ordinary and extraordinary modes of propagation. Birefringence therefore drastically reduces the enhancement of the EBS cone in the cross-polarized channel. Considering optical activity, the enhancement of the EBS cone in the helicity non-preserving channel would vanish for $\ell \gg \lambda / \Delta n$, where $\Delta n$ is the difference in refractive index for the two circular polarizations, and $\lambda$ the wavelength of light in vacuum. Applying this condition to the porous GaP samples implies $\Delta n \gg 1 / 2$, which, compared to an effective refractive index around 1.5 , seems unrealistically big.

\subsection{Conclusions}

A generalization of the diffusion equation to an anisotropic medium was presented. In the absence of a rigorous treatment of the boundary conditions of an anisotropic medium, only an anisotropic diffusion constant, along with an isotropic transport mean free path, can be considered. In this model where anisotropy is solely present in the diffusion constant, the theoretical expectations for all measurements, stationary and dynamic diffusion and EBS, depend on this dynamic anisotropy, in contrast with the isotropic case and intuition. Anisotropic samples were produced by electrochemical etching of GaP. Apart from the escape function, all optical measurements, both stationary and dynamic, on these samples were found to be anisotropic. The wave diffusion, in addition to the diffusion, is anisotropic in our samples. The anisotropy factor from all these measurements were found equal, within the experimental accuracy, to the square root of the anisotropy in diffusion constant. Our experimental results are therefore compatible with our theoretical predictions. 


\section{Chapter 7}

\section{Capturing a light pulse in a short high-finesse cavity}

The present chapter is set apart from the rest of the thesis since it does not deal with multiple scattering or porous gallium phosphide. This last chapter can be read independently from the previous chapters, and is self-explaining. Although originally not linked to multiple light scattering, the subject of capturing a light pulse in a cavity can be seen as a macroscopic equivalent of the switching of an (Anderson) localized state.

\subsection{Introduction}

There exist many examples of applications in optics where a combination of high (peak) intensity and narrow spectral width is desirable or even essential. One example is highresolution ultraviolet or vacuum ultraviolet spectroscopy [206,207] where second-harmonic generation ${ }^{1}(\mathrm{SHG})$ or four-wave mixing is needed to produce the appropriate wavelengths. A second example is laser isotope separation [207,209,210] where the yield depends on intensity but resolution is essential due to the smallness of isotope shifts. Generally speaking, high power and high resolution are mutually exclusive; the former belongs to the realm of pulsed lasers, the latter to the continuous-wave (cw) light sources. The present chapter describes a way in which this dichotomy can be circumvented: the aim is to obtain a light power characteristic of pulsed sources, yet at cw-bandwidths. An incident intense light pulse, captured right in a high-finesse cavity, offers both high intensity and narrow bandwidth.

It is simple to capture a short pulse in a cavity, i.e., when the duration of the pulse $\tau_{\mathrm{p}}$ is less than the round-trip time of the cavity $\tau_{\text {cav }}$. One can simply use an On/Off switch input coupler, similar to for example Q-switched lasers or regenerative amplifiers [211], to 'slam the door shut', after the pulse has entered. Because the incident pulse is fully captured inside the cavity, all its energy has to be distributed inside the resonances, the 'comb' of the cavity. The intensity of the light inside the cavity is enhanced at each resonance according to the number of round-trips before leaving the cavity.

\footnotetext{
1 and in particular intra-cavity SHG [208].
} 


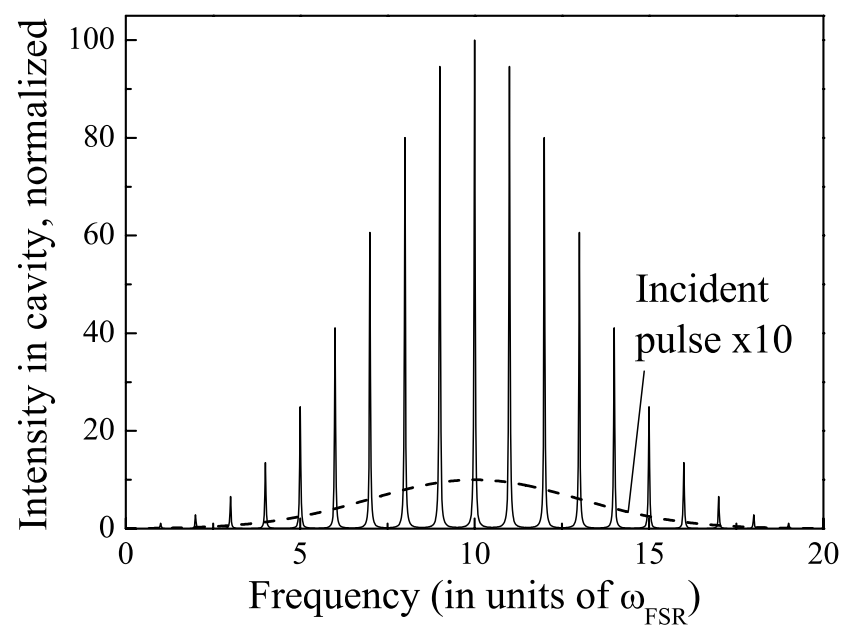

Figure 7.1: Calculated power spectrum of a short pulse captured inside a cavity of finesse $F=30$, normalized to the incident pulse peak power. The incident pulse is here multiplied by 10 for readability. The increase of intensity of the captured pulse at the resonances is due to the enhancement of the cavity itself and to the compression of the total energy of the incident pulse into the narrow peaks. The frequency scale is in units of the separation between the cavity resonances. See Fig. 7.6 and section 7.3 for the quantitative derivation.

In Fig. 7.1 (which is fully explained and derived in section 7.3), the power spectrum of a short pulse captured in a cavity of finesse $F=30$ is compared to the incident pulse. The power spectrum of the captured pulse is made of a number of narrow and intense peaks, at the resonant frequencies of the cavity. Each peak is narrow, but the series of peaks retains the original bandwidth of the pulse, i.e., the shape, or envelope, of the incident pulse is maintained in the cavity. If the pulse is filtered by, instead of captured in, a cavity of finesse $F=30$, the power spectrum of light inside the cavity keeps the same shape but is only increased by a factor 10 compared to the incident pulse.

To achieve the desired spectral narrowing, while retaining a high power, a short cavity is needed, i.e., $\tau_{\text {cav }}$ much smaller than $\tau_{\mathrm{p}}$. In the frequency domain the short cavity corresponds to the situation where only one cavity mode is overlapping with the pulse spectrum, in other words, the free spectral range (frequency separation between two resonances) of the cavity is larger than the pulse bandwidth $\Gamma$.

In order to capture the pulse, instead of filtering it through the cavity, the input mirror has to be switched, as mentioned in the case of a short pulse. Using an On/Off switch in order to capture a pulse in a short cavity is far from optimal: the switching can be done either too early or too late. Closed too early, the incident pulse has no chance to enter the cavity. Closed too late, most of the pulse enters the cavity, but leaks out immediately through the still-open input coupler. In this scheme, capturing a long pulse thanks to an instantaneous switch either compromises on obtained power, reduced bandwidth, or both.

The method described here [92] is based on the following idea: rather than using the variable input coupler as an open/close switch, the reflectivity of the input coupler is slowly changed from 0 to 1 on a time scale comparable to $\tau_{\mathrm{p}}$, hence much longer than $\tau_{\text {cav }}$. There 


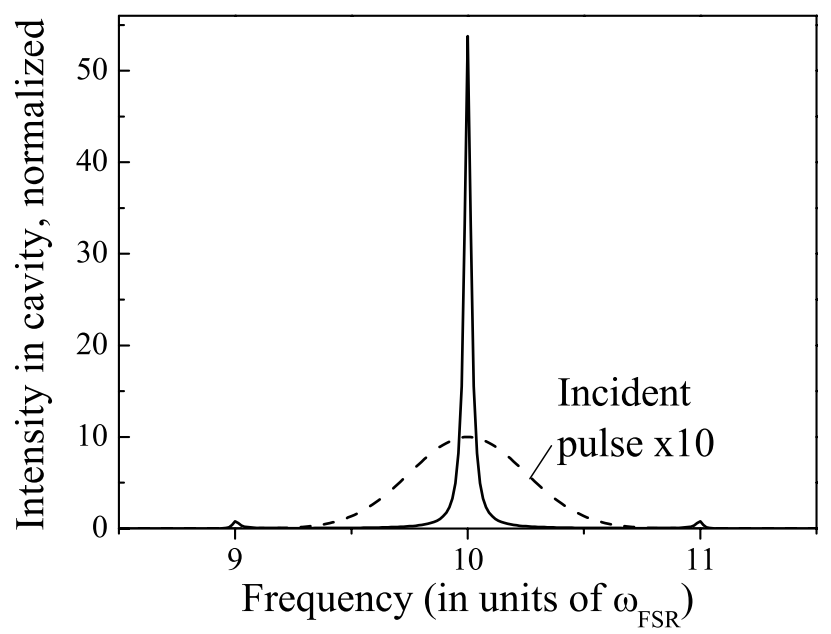

Figure 7.2: Calculated power spectrum of a long pulse captured inside a cavity of finesse $F=$ 30, normalized to the incident pulse peak power. The incident pulse is here multiplied by 10 for readability. Only one cavity mode overlaps with the incident pulse spectrum (although two extra modes are just visible, since also enhanced). The long pulse captured inside the cavity has a reduced bandwidth, but keeps all of its initial energy: the peak power is greatly enhanced.

exists an appropriate choice of switching function of the reflectivity which leads to zero loss: the value of the input reflectivity is chosen at each instant in time such that the fraction of the light already in the cavity that is transmitted back out exactly cancels the reflected fraction of the input pulse. This method can be called dynamic impedance matching.

Unlike the usual situation where a cavity acts as a filter [167], discarding the frequency components outside its resonant modes, capturing a pulse implies that all the pulse energy inside the cavity circulates at one of the resonance frequencies. The spectrum of the pulse has been compressed into the single available cavity mode, as depicted in Fig.7.2. In order to capture effectively the pulse inside the cavity, the knowledge of the shape and arrival time of the incident pulse as well as its central frequency is necessary. If implemented successfully the result of the scheme is remarkable: a pulse of light is turned into a nearly continuous-wave signal, of same energy, which decays on a time scale only limited by the finesse $F$ of the cavity.

The fact that the full energy is transferred in the cavity actually leads to an increase in power in the cavity relative to that of the incoming pulse, contrary to what one might guess. The reason is simply energy conservation: the peak power inside the cavity is the pulse energy divided by the cavity round-trip time $\tau_{\text {cav }}$, which by assumption is much smaller than the pulse duration $\tau_{\mathrm{p}}$.

The time to excite a mode inside a stationary cavity increases with the finesse. This incoupling time is in fact equal to the time $\tau_{\mathrm{r}}$ that light circulating within one mode of the stationary cavity needs to leak out via the non-perfect mirrors. In the case of a stationary high-finesse cavity, the time needed to excite one cavity mode is therefore much bigger than the round-trip time $\tau_{\text {cav }}$. Capturing a long light pulse in a cavity of high finesse, on the 
other hand, excites the single cavity mode within the switching time, i.e., within the pulse duration. The pulse duration is explicitly taken much smaller than the ring-down time $\tau_{\mathrm{r}}$ in this switching method. Exciting a single cavity mode is therefore much quicker in the dynamic, or switched, than in the stationary case.

Note that in the two examples shown in Figs. 7.1 and 7.2, respectively a short and long pulse captured in the same cavity, the enhancement in peak power is smaller in the long pulse case $(\sim 55)$ than in the short pulse case $(\sim 100)$. The enhancement arises from the compression of nearby frequency components into the resonant frequency. In the case of a Gaussian pulse for example, the power of the off-resonance frequency components quickly decreases. The best enhancement in power from a pulse captured in the same cavity would arise from a square-wave incident pulse: all the frequency components which can be compressed into the cavity mode are available in this incident pulse.

An essential element in the practical implementation of the scheme is the variable input coupler for which we use a combination of a polarizing beam splitter, a Pockels cell and polarization optics, along with precisely-timed switching electronics.

Along with the high-power and narrow-bandwidth applications already mentioned, a very different use of the scheme can be envisaged: reversible storage and subsequent release of a light pulse with almost $100 \%$ efficiency. As the method is essentially adiabatic, the stored light can readily be released again by reversing the process. The holding time is limited only by the finesse of the closed cavity, i.e., how many round-trips light can travel before leaking out through the non-perfect mirrors. This capture and release application bears some analogy with experiments that have attracted considerable attention recently. In papers from two different groups [212,213] it was shown that light could be effectively 'slowed down' to zero velocity and stored in a medium of atomic vapor. Those experiments use a control field to transform the character of a coupled light-atom coherence from almost purely light-like to almost atomic, in a reversible adiabatic manner. The capture of pulses in a cavity proceeds along somewhat analogous lines. The 'atomic-like' state of refs. [212] and [213] can be compared to the cavity with the light captured inside. Clearly the analogy is far from complete as the experiment presented here is purely classical. One important practical consequence of this difference is that, unlike the atom, the narrow-band light inside the cavity is actually available for intra-cavity experiments, e.g., intra-cavity SHG. Note that, in order to perform such capture and release experiments, an improved version of our experiment, which is presented here as a proof of principle, is required.

Another scheme for all-optical stopping and storing of light has been presented [214, 215]. An array of micro-resonators was shown, theoretically and numerically, to slow down a pulse of light to arbitrarily small group velocities.

The following section of this chapter contains an introduction to the theoretical description of a pulse in a stationary cavity. Section 7.3 makes the theoretical step to the dynamic cavity, quantitatively describing the capture scheme, for both short and long pulse. In sections 7.4 and 7.5 the special input coupler and our experimental methods are discussed. In section 7.6 the experimental results are presented and analyzed. 
Figure 7.3: Schema of a ring cavity with 2 high reflectivity mirrors $M_{3}$ and $M_{4}$, a leaking mirror $M_{2}$ of (field) reflectivity $\alpha$ and an input coupler $M_{i}$ of (field) reflectivity $\rho$. The intensity reflectivity $R \equiv \rho^{2}$ of the input coupler can be time-dependent, as is described in section 7.3 .

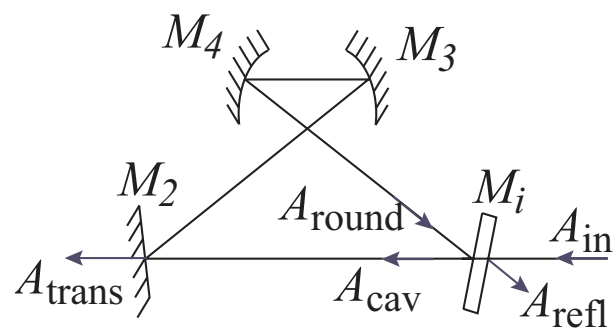

\subsection{Introduction to the theory of a cavity}

Consider a ring cavity ${ }^{2}$ in which light travels in one direction, as sketched in Fig. 7.3 Four waves are coupled with each other by the input coupler $M_{i}$ : the incident field $A_{\mathrm{in}}$, the reflected field $A_{\text {refl }}$, the field just after entering the cavity $A_{\text {cav }}$, and the field in the cavity after one round-trip, back on the input coupler $A_{\text {round }}$. The complex fields $A_{\text {round }}$ and $A_{\text {in }}$ are reflected by the input coupler according to the reflection coefficient $\rho$. The mirror $M_{2}$ has a field reflectivity $\alpha$ smaller than 1 and lets light leak out of the cavity. The leakage through $M_{2}$ can also represent the losses of the cavity. The leakage through $M_{2}$ is in fact transmitted by the cavity, and its field is denoted $A_{\text {trans }}$. The optical length of the cavity is taken as $L$ and the leakage mirror $M_{2}$ can be taken at any optical length $L_{t}$ smaller than $L$.

All fields are considered in the plane-wave approximation ${ }^{3}$ Pulses are assumed to be Fourier-transform limited ${ }^{4}$ The incident field for example is

$$
A_{\text {in }}(t, z)=\int S(\omega) \exp [-i(\omega t-k z)] \mathrm{d} \omega,
$$

where $S(\omega)$ is the frequency spectrum, $\omega$ and $k=\omega / c$ respectively the frequency and wave vector of the incident light. The incident field oscillates in time $t$ and in the coordinate $z$ of its propagation direction.

The two fields leaving the input coupler, $A_{\text {cav }}$ and $A_{\text {refl }}$, are each a sum of the two fields impinging on $M_{i}$, weighted by the proper transmission and reflection coefficients. The field in the cavity just before the input coupler, $A_{\text {round }}$, is the remainder of the field, reflected from the leakage mirror, after traveling one round-trip. The transmitted field, $A_{\text {trans }}$, is the transmission through the leakage mirror $M_{2}$ of the light inside the cavity.

\footnotetext{
${ }^{2}$ Another possibility is a linear cavity, where light is reflected on the same path back. The modes of a linear cavity are made of stationary waves. The theory of a pulse capture inside a linear cavity can also be done, but is not detailed in this thesis.

${ }^{3}$ In fact, the complex field amplitudes $A_{i}$ should be taken as the amplitude in the center of a Gaussian mode. The waist of the Gaussian modes are equal for the four fields at the input coupler, and can be taken equal to the waist of the mode at the leakage mirror.

${ }^{4}$ In the actual experiment, in section 7.5 this assumption is not strictly true. Yet, the results in section 7.6 will empirically show that the method presented here is still applicable to pulses which are not Fourier-limited.
} 
The coupling of all the fields is therefore

$$
\begin{aligned}
A_{\text {cav }}(t) & =\sqrt{1-\rho^{2}(t)} A_{\text {in }}(t)+\rho(t) A_{\text {round }}(t) \\
A_{\text {refl }}(t) & =\sqrt{1-\rho^{2}(t)} A_{\text {round }}(t)-\rho(t) A_{\text {in }}(t) \\
A_{\text {round }}(t) & =\alpha A_{\text {cav }}\left(t-\tau_{\text {cav }}\right) \\
A_{\text {trans }}(t) & =\sqrt{1-\alpha^{2}} A_{\text {cav }}\left(t-L_{t} / c\right),
\end{aligned}
$$

where $\tau_{\text {cav }} \equiv L / c$ is the round-trip time of the cavity. In principle the field reflectivities $\rho$ and $\alpha$ are complex but are here set as positive without loss of generality. Note that the field reflection of light by the input coupler changes sign with the reflecting side. At an interface glass-air for example, the Fresnel reflection coefficient is positive for the reflection on the glass side, and negative on the air side [167]. The reflectivity of the input coupler $\rho(t)$ is here written in the most general form as time-dependent, which gives the obvious followup to the next, dynamic, section. The leakage reflectivity $\alpha$ is kept constant, in the present and following section alike.

The field of light inside the cavity follows a recurrent equation which can easily be solved:

$$
\begin{aligned}
A_{\mathrm{cav}}(t)= & \sqrt{1-\rho^{2}(t)} A_{\mathrm{in}}(t)+\alpha \rho(t) A_{\mathrm{cav}}\left(t-\tau_{\mathrm{cav}}\right) \\
A_{\mathrm{cav}}(t)= & \sqrt{1-\rho^{2}(t)} A_{\mathrm{in}}(t) \\
& \quad+\sum_{n=1}^{\infty}\left[\alpha^{n} \sqrt{1-\rho^{2}\left(t-n \tau_{\mathrm{cav}}\right)} A_{\mathrm{in}}\left(t-n \tau_{\mathrm{cav}}\right) \prod_{m=0}^{n-1} \rho\left(t-m \tau_{\mathrm{cav}}\right)\right] .
\end{aligned}
$$

The reflected and transmitted light from the cavity are related to the light inside the cavity according to

$$
\begin{aligned}
A_{\text {refl }}(t) & =\alpha \sqrt{1-\rho^{2}(t)} A_{\text {cav }}\left(t-\tau_{\text {cav }}\right)-\rho A_{\text {in }}(t) \\
A_{\text {trans }}(t) & =\sqrt{1-\alpha^{2}} A_{\text {cav }}(t) \exp \left(i k L_{t}\right) .
\end{aligned}
$$

According to Eq.7.7, the field of light inside the cavity is linearly related to the incident field. Likewise, both transmitted and reflected fields are linearly related to the incident field. The cavity is shown to 'scatter' the incident light from $\mathbf{k}_{\text {in }}=k \hat{\mathbf{z}}$ to $\mathbf{k}_{\text {trans }}$ and $\mathbf{k}_{\text {refl }}$, with scattering coefficients equal to $A_{\text {refl/trans }}(t) / A_{\text {in }}\left(t-n \tau_{\text {cav }}\right)$ and scattering delay $n \tau_{\text {cav }}\left(+L_{t} / c\right.$ in transmission). In the language of scattering, used till this chapter, we just obtained a T-matrix for this cavity scatterer.

\subsubsection{Stationary cavity}

In the case of a usual, stationary, cavity where $\rho$ does not depend on time, the solution is found as

$$
A_{\text {cav }}(t)=\sqrt{1-\rho^{2}} \sum_{n=0}^{\infty} \alpha^{n} \rho^{n} A_{\text {in }}\left(t-n \tau_{\text {cav }}\right) .
$$


Developing the incident light into its Fourier components (see Eq.7.1 for $z=0$ ) gives

$$
\begin{aligned}
& A_{\text {cav }}(t)=\sqrt{1-\rho^{2}} \sum_{n=0}^{\infty}\left\{\alpha^{n} \rho^{n} \int S(\omega) \exp (-i \omega t) \exp \left(i \omega n \tau_{\text {cav }}\right) \mathrm{d} \omega\right\} \\
& A_{\text {cav }}(t)=\sqrt{1-\rho^{2}} \int \frac{S(\omega) \exp (-i \omega t)}{1-\alpha \rho \exp \left(i \omega \tau_{\text {cav }}\right)} \mathrm{d} \omega,
\end{aligned}
$$

where the sum and the integral can be swapped due to the uniform convergence of the Fourier transform. The subsequent geometrical series is summed under the assumption that $\left|\alpha \rho \exp \left(i \omega \tau_{\text {cav }}\right)\right|<1$, which is always true in a passive realistic cavity.

The frequency spectrum in the stationary cavity is the inverse Fourier transform of $A_{\text {cav }}(t)$ :

$$
A_{\text {cav }}(\omega)=\frac{S(\omega) \sqrt{1-\rho^{2}}}{1-\alpha \rho \exp \left(i \omega \tau_{\text {cav }}\right)} .
$$

In the case of a monochromatic source, for $S(\omega)=S_{0} \delta\left(\omega-\omega_{0}\right)$, the usual [167] 'cavity comb' is retrieved for the intensity of light inside the cavity: in the case of a good cavity, i.e., $\rho$ and $\alpha$ close to 1 , the power spectrum in the stationary cavity is very small except for discrete frequencies, the resonances, where the intensity is greatly increased by the cavity.

$$
I_{\text {cav }}(\omega) \equiv\left|A_{\text {cav }}(\omega)\right|^{2}=\frac{\left(1-\rho^{2}\right)|S(\omega)|^{2}}{\left|1-\alpha \rho \exp \left(i \omega_{0} \tau_{\text {cav }}\right)\right|^{2}} .
$$

A resonance of the cavity appears every time $\alpha \rho \exp \left(i \omega_{0} \tau_{\text {cav }}\right)$ approaches 1 . The bandwidth of the resonance is then dependent on the reflectivity coefficients of the mirrors. The free spectral range is the frequency separation between two resonances $\omega_{\mathrm{FSR}}$. The finess 5 of a cavity is defined as the ratio of the free spectral range and the bandwidth (or full width at half maximum) of a resonance.

$$
F \equiv \frac{\omega_{\mathrm{FSR}}}{\omega_{\mathrm{r}}}=\frac{\pi}{2} \arcsin ^{-1}\left(\frac{1-\rho \alpha}{2 \sqrt{\rho \alpha}}\right) .
$$

Fig. 7.4 shows the power spectrum of a pulse, respectively transmitted and reflected by a cavity of finesse $F=30$. The power spectrum of the light inside the cavity is found according to Eq.7.9 and is equal to the transmitted power spectrum multiplied by $1 /\left(1-\alpha^{2}\right)$.

It is easily checked that the sum of the power spectrum reflected by and transmitted through the cavity exactly equals the incident power spectrum (see Fig. 7.4). The scattering of the cavity is elastic, or in other words, the T-matrix of the cavity fulfills the optical theorem at each frequency. The stationary cavity is a filter: only the frequency components of the incident pulse within the resonances of the cavity are transmitted, whereas the rest does not penetrate the cavity and is reflected.

\footnotetext{
${ }^{5}$ In other fields of physics, another important parameter is the quality factor (or Q-factor). The Q-factor is characteristic of each resonance of a cavity or resonator. The Q-factor is defined as the ratio of the frequency and the bandwidth of a resonance.
} 


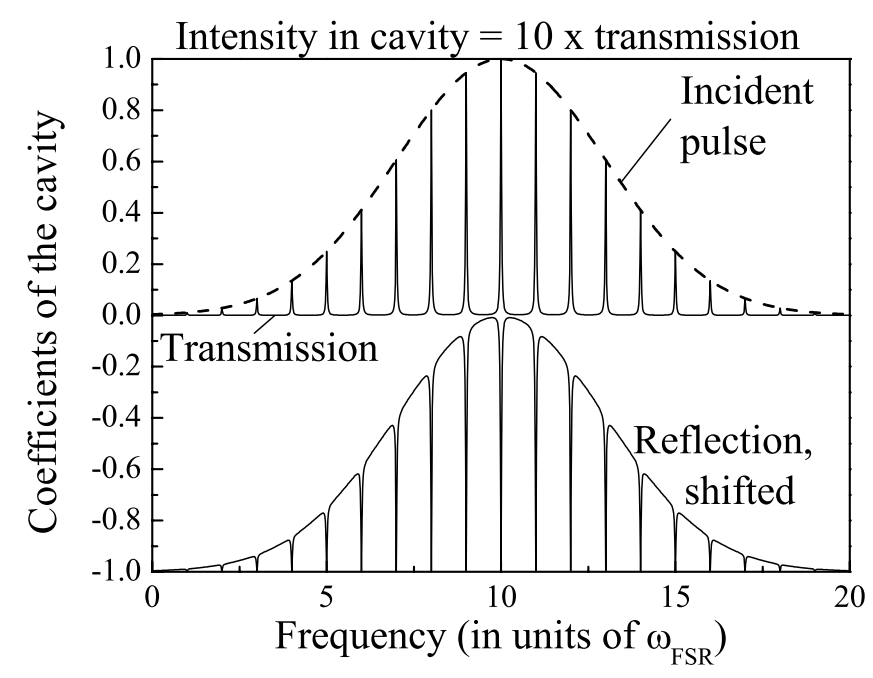

Figure 7.4: Normalized power spectrum in transmission and reflection of a pulse of duration smaller than the cavity round-trip time. In transmission, only the frequency components of the incident pulse which are within the resonances of the cavity remain. In reflection (shifted down by 1 for readability) remains the rest of the incident spectrum. The cavity has a finesse $F=30$, with $\rho=\alpha=\sqrt{0.9}$. The power spectrum of the light inside the cavity is equal to the transmitted spectrum multiplied by $1 /\left(1-\alpha^{2}\right)=10$. The frequency scale is in units of the free spectral range.

Taking a Lorentzian frequency spectrum for the incident light, the bandwidth $W_{\mathrm{FWHM}}$ of the spectral peaks inside the cavity is given for later reference:

$$
\begin{aligned}
S(\omega) & =\frac{S_{0} \Gamma}{\left(\omega-\omega_{0}\right)^{2}+(\Gamma / 2)^{2}} \\
W_{\mathrm{FWHM}} & =\frac{1}{\sqrt{2}} \sqrt{\sqrt{\left(\Gamma^{2}+\omega_{\mathrm{r}}^{2}\right)^{2}+4 \omega_{\mathrm{r}}^{2} \Gamma^{2}}-\Gamma^{2}-\omega_{\mathrm{r}}^{2}} .
\end{aligned}
$$

\subsection{Theoretical description of the pulse capture}

The usefulness of a cavity is obvious: in transmission, or inside the cavity, a power spectrum with sharp resonances is available, where the sharpness is only limited by the reflectivity coefficients of the mirror. The drawback of a cavity is also obvious: all the gain in narrow bandwidth implies a loss in power, namely the filtering out of the off-resonance frequency components.

In order to couple the full incident power in the cavity, the input coupler is dynamically closed. The reflectivity function of the input coupler is changed so that the intensity reflected by the cavity vanishes at all time (see Eq.7.8. 


$$
\begin{aligned}
0=A_{\text {refl }}(t) & =\alpha \sqrt{1-\rho^{2}(t)} A_{\text {cav }}\left(t-\tau_{\text {cav }}\right)-\rho(t) A_{\text {in }}(t) \\
\Longleftrightarrow \rho^{2}(t) & =\frac{\alpha^{2} A_{\mathrm{cav}}^{2}\left(t-\tau_{\text {cav }}\right)}{A_{\text {in }}^{2}(t)+\alpha^{2} A_{\text {cav }}^{2}\left(t-\tau_{\text {cav }}\right)} .
\end{aligned}
$$

This dynamic reflectivity function of the input coupler allows the full capture of any incident pulse, short or long, inside the cavity.

\subsubsection{Capturing a short pulse}

In the case of a short pulse $\left(\tau_{\mathrm{p}} \ll \tau_{\text {cav }}\right)$, when most of the time both intensities just before and just after the input coupler $\left(A_{\text {in }}\right.$ and $\left.A_{\text {cav }}\right)$ vanish, the reflectivity can be chosen by hand as a step function:

$$
\rho_{s}(t)=\rho_{s}^{2}(t)=\Theta\left(t-t_{s}\right), \text { where } \tau_{\mathrm{p}} \ll t_{s}, \tau_{c a v}-t_{s} .
$$

The origin of time is set as the moment when the center of the pulse passes the input coupler. The Heaviside function $\Theta\left(t-t_{s}\right)$ makes the reflectivity 0 before the pulse arrives, leaving the cavity fully open, and makes the reflectivity $100 \%$ after the pulse has entered. The switch timing $t_{s}$ should be after the full pulse has entered and before the return of the pulse on the input coupler after one round-trip. Typically $t_{s} \sim \tau_{\text {cav }} / 2$.

Using the Heaviside reflectivity function in the exact function for $A_{\text {cav }}$ (Eq. 7.7) gives

$$
\begin{aligned}
A_{\mathrm{cav}}(t)= & {\left[1-\Theta\left(t-t_{s}\right)\right] A_{\text {in }}(t) } \\
& +\sum_{n=1}^{\infty}\left\{\alpha^{n}\left[1-\Theta\left(t-n \tau_{\mathrm{cav}}-t_{s}\right)\right] A_{\text {in }}\left(t-n \tau_{\text {cav }}\right) \prod_{m=0}^{n-1} \Theta\left(t-m \tau_{\text {cav }}-t_{s}\right)\right\} .
\end{aligned}
$$

The product of the Heaviside function is 0 as soon as one of the functions (for $0 \leq$ $m \leq n-1)$ is 0 . The $n^{\text {th }}$ term in the sum therefore vanishes for $t-(n-1) \tau_{c a v}-t_{s} \leq$ $t-m \tau_{c a v}-t_{s}<0$, i.e., $n>1+\left(t-t_{s}\right) / \tau_{\text {cav }}$. Similarly the $n^{\text {th }}$ term in the sum is zero for $t-n \tau_{c a v}-t_{s} \geq 0$, i.e., $n \leq\left(t-t_{s}\right) / \tau_{c a v}$. The only non-zero term in the sum is therefore such that $\left(t-t_{s}\right) / \tau_{c a v}<n_{s} \leq 1+\left(t-t_{s}\right) / \tau_{c a v}$. The sum in Eq.7.21 reduces to one term which is

$$
\left\{\begin{array}{l}
A_{\mathrm{cav}}(t)=\alpha^{n_{s}} A_{\mathrm{in}}\left(t-n_{s} \tau_{\mathrm{cav}}\right) \\
n_{s}=\text { floor }\left(1+\frac{t-t_{s}}{\tau_{\mathrm{cav}}}\right) \geq 0,
\end{array}\right.
$$

where floor $(x)$ is the largest integer smaller or equal to $x$. Remember that the sum in Eq.7.21 is for $n$ positive, therefore $n_{s}$ must also be positive. This condition of $n_{s}$ positive (or equivalently $t \geq t_{s}-\tau_{\text {cav }}$ ) insures the physical need of having no light inside the cavity before the pulse has entered.

As seen from Fig. 7.5, which is the intensity of a short pulse captured in the cavity, the pulse just runs around in the cavity, without interfering with itself. The overall intensity of the pulse in the cavity is reduced each round-trip by $(1-\alpha)$, leaking out through the non-perfect mirror. 


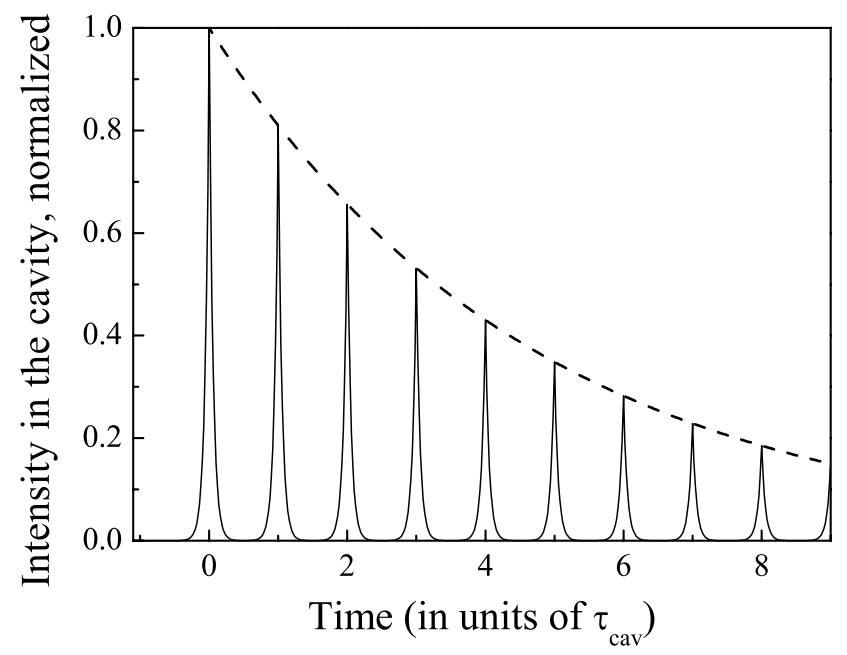

Figure 7.5: Time-resolved intensity of light of a short pulse captured in the cavity. The incident pulse matches the first peak at $t=0$ since it is fully coupled in the cavity. The pulse then runs around in the closed cavity of finesse $F=30$, only leaking through the non-perfect mirror, where $\alpha=\sqrt{0.81}$. The dashed curved is $\alpha^{2 t / \tau_{\mathrm{cav}}}$.

The frequency spectrum of the short pulse inside the cavity is

$$
A_{\text {cav }}(\omega)=\int_{t_{s}-\tau_{\text {cav }}}^{\infty} \alpha^{n_{s}} A_{\text {in }}\left(t-n_{s} \tau_{\text {cav }}\right) \frac{\exp (i \omega t)}{2 \pi} \mathrm{d} t
$$

In order to solve this integral, the time origin is shifted by $t_{s}$, and the integral is split into an infinite sum of integrals:

$$
\int_{-\tau_{\mathrm{cav}}}^{\infty}=\int_{-\tau_{\mathrm{cav}}}^{0}+\int_{0}^{\tau_{\mathrm{cav}}}+\cdots+\int_{n \tau_{\mathrm{cav}}}^{(n+1) \tau_{\mathrm{cav}}}+\cdots=\sum_{n=-1}^{\infty} \int_{n \tau_{\mathrm{cav}}}^{(n+1) \tau_{\mathrm{cav}}} .
$$

In each component of the sum of integrals, the origin of time is taken independently so that all integrals span the same range $\left[0 ; \tau_{\text {cav }}[\right.$. Then

$$
A_{\text {cav }}(\omega)=\exp \left(i \omega t_{s}\right) \sum_{n=-1}^{\infty}\left[\alpha^{n+1} \exp \left(i \omega n \tau_{\text {cav }}\right)\right] \int_{0}^{\tau_{\text {cav }}} A_{\text {in }}\left(t+t_{s}-\tau_{\text {cav }}\right) \frac{\exp (i \omega t)}{2 \pi} \mathrm{d} t .(7
$$

The geometrical series gives rise to the expected cavity comb:

$$
A_{\text {cav }}(\omega)=\frac{\exp \left(i \omega t_{s}\right)}{2 \pi} \frac{\exp \left(-i \omega \tau_{\text {cav }}\right)}{1-\alpha \exp \left(i \omega \tau_{\text {cav }}\right)} \int_{0}^{\tau_{\text {cav }}} A_{\text {in }}\left(t+t_{s}-\tau_{\text {cav }}\right) \exp (i \omega t) \mathrm{d} t .
$$

The frequency spectrum of the short pulse captured in the cavity is therefore the product of the cavity comb and a term depending on the incident pulse.

The spatial shape of an incident pulse of Gaussian frequency spectrum is also Gaussian:

$$
\begin{aligned}
A_{\text {in }}(t) & =\int_{-\infty}^{\infty} \exp \left[-\frac{\left(\omega-\omega_{0}\right)^{2}}{4 \Gamma^{2}}\right] \exp (-i \omega t) \mathrm{d} \omega \\
& =2 \sqrt{\pi} \Gamma \exp \left(-i \omega_{0} t\right) \exp \left(-\Gamma^{2} t^{2}\right)
\end{aligned}
$$




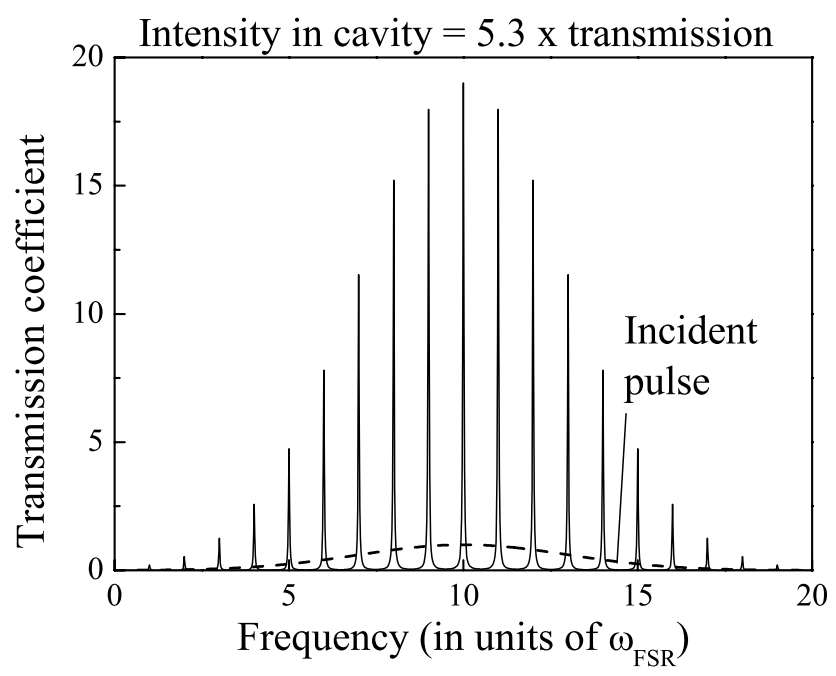

Figure 7.6: Normalized power spectrum in transmission of a short pulse captured in a cavity of finesse $F=30$, with $\alpha=\sqrt{0.81}$. The switched cavity is not a filter: the frequency components of the incident pulse are compressed inside the resonances of the closed cavity. The power spectrum of the light inside the cavity is equal to the transmitted spectrum multiplied by $1 /\left(1-\alpha^{2}\right) \simeq 5$.3. The frequency scale is in units of the free spectral range.

The field in the dynamic long cavity $A_{\text {cav }}(\omega)$ can be made explicit in the case of such a Gaussian input pulse:

$$
\begin{aligned}
& A_{\text {cav }}(\omega)=\frac{1 / 2}{1-\alpha \exp \left(i \omega \tau_{\text {cav }}\right)} \exp \left[-\frac{\left(\omega-\omega_{0}\right)^{2}}{4 \Gamma^{2}}\right] \\
& \times\left\{\operatorname{erf}\left(\Gamma t_{s}-i \frac{\omega-\omega_{0}}{2 \Gamma}\right)-\operatorname{erf}\left[\Gamma\left(t_{s}-\tau_{\text {cav }}\right)-i \frac{\omega-\omega_{0}}{2 \Gamma}\right]\right\},
\end{aligned}
$$

where $\operatorname{erf}(x)$ is the so-called error function which, for any $x \in \mathbb{C}$, has the property

$$
\operatorname{erf}(\Gamma x) \equiv \frac{2 \Gamma}{\sqrt{\pi}} \int_{0}^{x} \exp \left(-\Gamma^{2} t^{2}\right) \mathrm{d} t .
$$

The transmitted intensity through the cavity is related to the intra-cavity intensity by a ratio $\left(1-\alpha^{2}\right)$ (see Eq. 7.9). The reflection of the switched cavity vanishes, as is specified by Eq. 7.18 Fig. 7.6 shows the transmission coefficient through a switched cavity of finesse $F=30$. The cavity after switching is therefore comparable to the stationary cavity shown in Fig. 7.4. Although the total energy of the incident pulse is conserved after transmission through the switched cavity, its power spectrum has been modified. Frequency components which can not exist in the cavity, i.e., outside resonances, are adiabatically pushed toward the resonant frequencies. The switched cavity is not an elastic scatterer. Note that the overall bandwidth of the captured pulse is not reduced, but the spectral brightness (the power at a single frequency) is greatly increased at the resonance frequencies. Fig. 7.1 in the introduction of this chapter is the intra-cavity intensity corresponding to the transmission shown in Fig.7.6. 
If the same capture scheme is possible when only one resonance of the cavity overlaps with the incident pulse spectrum, i.e., for a long pulse, we expect a real narrowing of the bandwidth along with an increase of the power at the resonance frequency.

\subsubsection{Capturing a long pulse}

In the case of an incident pulse of duration of same order or larger than the round-trip time, the step reflectivity function considered in the previous section (Eq. 7.20) can not be used. The optimal reflectivity function, depending on the exact intensity inside and incident on the cavity, is Eq.7.19. Substituting the optimal reflectivity function in the equation for $A_{\text {cav }}(t)$ (Eq.7.7) gives a non-linear recurrent equation for which no analytical solution could be found.

A numerical simulation of the short cavity case can be made to gain some feeling about the behavior of the capture scheme. Such a simulation can be done in the time domain, since the switching function of the input coupler reflectivity is adapted to the temporal shape of the incident pulse. However, considering the experiments, the main goal of the method is to provide a spectral narrowing of the captured light and measurement of this narrowing is best performed as directly as possible, hence in the frequency domain.

Combining Eqs.7.6 and 7.18, we obtain an equation on the amplitude of the fields:

$$
\left|A_{\text {cav }}(t)\right|^{2}=\left|A_{\text {in }}(t)\right|^{2}+\alpha^{2}\left|A_{\text {cav }}\left(t-\tau_{\text {cav }}\right)\right|^{2} .
$$

In the case of a pulse of duration much larger than the round-trip time, the intensity inside the cavity does not change much during one round-trip, and a first-order Taylor expansion can be used:

$$
\frac{\mathrm{d}}{\mathrm{d} t}\left|A_{\mathrm{cav}}(t)\right|^{2}=\frac{\left|A_{\text {in }}(t)\right|^{2}}{\tau_{\text {cav }}}-\frac{\left|A_{\text {cav }}(t)\right|^{2}}{\tau_{\mathrm{r}}},
$$

where $\tau_{\mathrm{r}} \equiv \tau_{\text {cav }} /\left(1-\alpha^{2}\right)$ is the ring-down time of the cavity, or how long light stays in the cavity before leaking out.

Let us first consider an ideal cavity without losses so that $\tau_{\mathrm{r}}^{-1}$ vanishes, or equivalently of infinite finesse. The condition of vanishing reflectivity (Eq. 7.18) being fulfilled, the intensity inside the cavity is the integral of the incident pulse:

$$
\tau_{\text {cav }}\left|A_{\text {cav }}(t)\right|^{2}=\int_{-\infty}^{t} \mathrm{~d} \tau\left|A_{\text {in }}(\tau)\right|^{2} .
$$

According to Eqs. 7.19 and 7.32 for a given input pulse shape, the reflectivity function $R(t) \equiv \rho^{2}(t)$ appropriate for coupling all the energy into the cavity can be determined.

Fig. 7.7 a shows an example of capture of a Gaussian pulse of unit width and height in a cavity with $\tau_{\text {cav }}=0.086$. The ratio of $\tau_{\mathrm{p}}$ and $\tau_{\text {cav }}$ matches the experimental value in section 7.5. In the lossless case, after switching, the cavity is completely closed and the light circulates forever, with an intensity which exceeds that of the peak of the incident pulse by more than an order of magnitude. In the cavity of infinite finesse, light travels therefore in a single, infinitely narrow, frequency band around the cavity resonance frequency which coincides with the input pulse frequency. The effect of switching the input coupler is to adiabatically change the spectrum from the initial Fourier transform of the pulse shape 

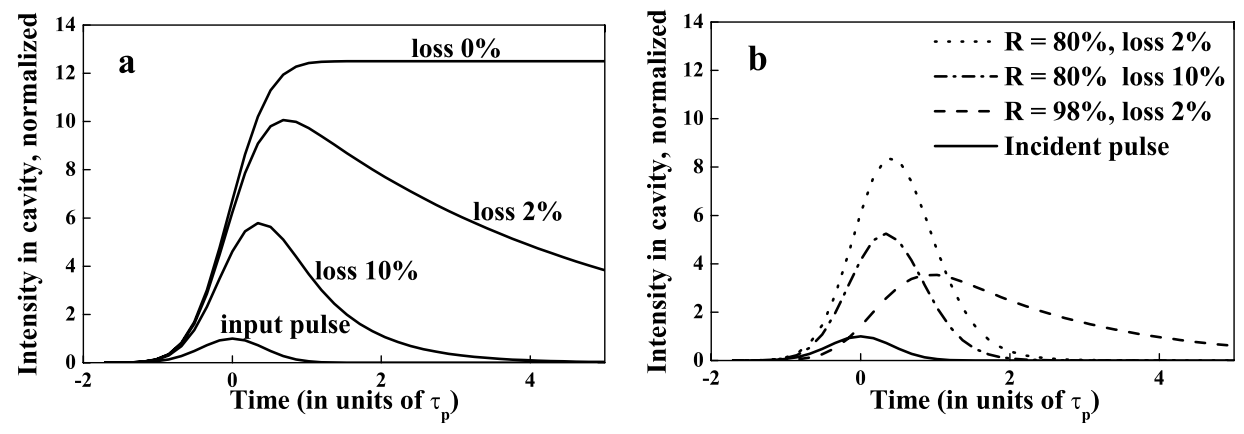

Figure 7.7: Intensity inside the cavity as a function of time for a Gaussian input pulse of unit width and height, and $\tau_{\text {cav }}=0.086$. In (a), curves are compared for the optimally switched reflectivity, in a cavity with various losses. The switching scheme captures the light pulse. In (b), the curves correspond to the same cavity but with a fixed reflectivity of $M_{i}$, for $R=80 \%$ and $R=98 \%$. The solid curves correspond to $2 \%$ loss. The dashed curve corresponds to $10 \%$ loss and $R=80 \%$. The light in a stationary cavity never reaches the same bandwidth and power characteristics as in the switched case.

into the Lorentzian cavity spectrum. The frequency components of the pulse are gradually pushed closer to the central cavity mode as the cavity finesse increases, i.e., as the cavity closes, or the reflectivity of the input coupler increases.

The use of Gaussian pulses is not essential. In principle the switching method works even for a square-wave pulse if its duration exceeds $\tau_{\text {cav }}$. In the case of an incident square pulse the time-dependent reflectivity of $M_{i}$ changes in small discrete step $\S^{6}$ every time the leading edge of the pulse inside the cavity arrives back at the input coupler.

A discussion of how in practice the cavity length is matched to the central frequency of the pulse is deferred to section 7.5. In section 7.4 the possibility to allow for a modest chirp rather than using purely transform limited pulses is commented on.

The requirement that the losses are zero is now relaxed. Even with very good components a realistic minimum power loss is $2 \%$ per round-trip. Thus $\tau_{\mathrm{r}} \approx 50 \tau_{\text {cav }}$. Eqs. 7.19 and 7.31 can also be numerically solved when including finite losses. The results of the optimally switched cavity for both $2 \%$ and $10 \%$ loss are shown in Fig. 7.7 A. For the same $R(t)$ but different losses the stored intensity now decays on a time scale characteristic of the additional loss.

Note that in all cases of the switched cavity, in Fig. 7.7 , the mode inside the cavity is fully excited with a typical time comparable to the pulse duration. The ring-down time $\tau_{\mathrm{r}}$, or equivalently the time to excite a single mode of the equivalent stationary cavity, is set by the losses. At a typical loss of $2 \%$ for example, the decay of the intensity inside the switched cavity is much slower than the time needed to excite the cavity mode. The cavity mode can therefore be excited much more quickly in a dynamic than in a stationary cavity. The inherent slowness of a high-finesse stationary cavity can be avoided by switching the cavity, for any captured pulse of duration smaller than the ring-down time.

In Fig. $7.7 \mathrm{p}$ the intra-cavity intensity versus time for two fixed reflectivities of the input

\footnotetext{
${ }^{6}$ Such small steps are unpractical to implement in the time-scales considered in section 7.5
} 

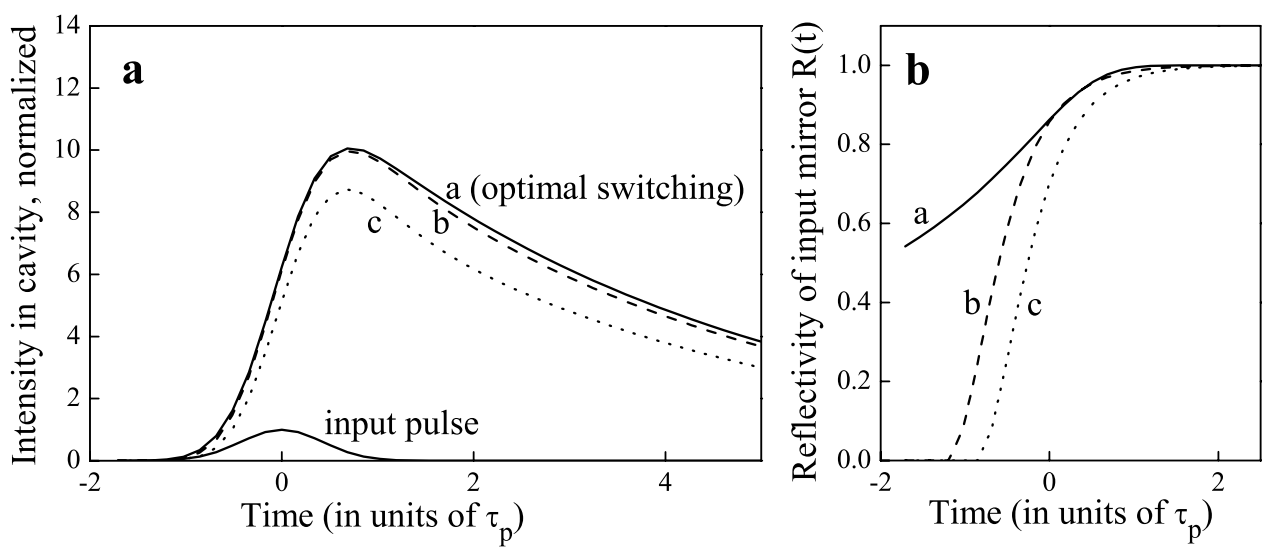

Figure 7.8: In (a), intensity in the cavity as a function of time for the same parameters as in Fig. 7.7 Each curve (full, dashed, and dotted) corresponds to a different reflectivity switching function, plotted in (b). The full curves correspond to the optimal switching of the cavity. The dashed curves correspond to a switching function experimentally easy to implement. The dotted curves correspond to the same experimental reflectivity function as for the dashed curves, with a shift in timing. All curves are for a cavity with $2 \%$ loss. The switching scheme is not very sensitive to the exact switching function.

coupler are shown for a cavity with $2 \%$ loss. Two cases are presented: $R=80 \%$, which corresponds to the highest possible peak intensity, and $R=98 \%$. The former curve $(R=$ $80 \%$, loss $2 \%$ ) has a peak power comparable to the switched case (loss $2 \%$ in Fig. 7.7 f), but a faster decay, implying a larger bandwidth of the light in the fixed cavity. The latter curve ( $R=98 \%$, loss $2 \%$ ) decays nearly as slowly as in the switched case but the peak amplitude is much smaller. For higher loss rates the advantage of the time-dependent $R$ diminishes: for $10 \%$ loss the slower decaying tail in the switched case (loss $10 \%$ in Fig. 7.7 ) than in the constant reflectivity case $(R=80 \%$, loss $10 \%$ in Fig. 7.7 a) is still just visible. This loss rate of $10 \%$ marks the boundary of the usefulness of the switching reflectivity method and corresponds to a ring-down time of the order of the pulse duration.

A comparison of Figs. $7.7 \mathrm{p}$ and $7.7 \mathrm{p}$ indicates that for any fixed reflectivity of the input coupler the intra-cavity intensity decays faster than for the switched case for given loss. As stated before this faster decay implies that the power spectrum in the switched case is always narrower than in the stationary case.

One may well ask how critically the exact temporal behavior of $R(t)$ determines the fraction of light that can be coupled into the cavity. As a check, the perfect no-reflection condition Eq.7.18 is relaxed and the optimal switching function $R(t)$ is replaced by a function which results from an exponentially decreasing voltage on the Pockels cell used to implement the switching mirror, as described in the next section. Such an exponentially decreasing voltage leads to the following dependence of the effective reflectivity:

$$
\begin{aligned}
& R(t)=\frac{1}{1+\tan ^{2} \theta(t)}, \\
& \text { with } \theta(t)=(\pi / 2)\left\{1-\Theta(t)\left[1-\exp \left(-t / \tau_{\mathrm{s}}\right)\right]\right\},
\end{aligned}
$$

where $\tau_{\mathrm{s}}$ is the relaxation time of the Pockels cell and switching electronics. The switch 
Figure 7.9: The input coupler consisting of a polarizing beam splitter BS, a Pockels cell, two half-wave plates $\lambda / 2$, two quarter-wave plates $\lambda / 4$. The full lines on the wave plates indicate the orientation of the optical axes. The full arrows indicate the direction of polarization. The light inside the cavity is transmitted through BS, and the incident light, of crossed polarization, is reflected on $B S$. The voltage on the Pockels cell is chosen so that the sum of the cavity and incident fields is always rotated back into the cavity mode, of horizontal polarization.

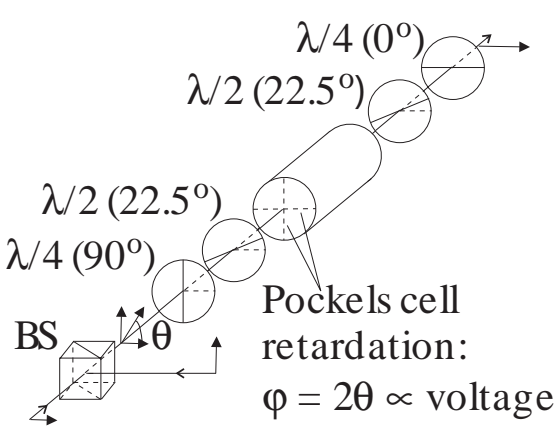

is described in detail in section 7.5 In Fig. 7.8p, three reflectivity functions are plotted, the optimal switching function (in full curve) and two functions described by Eq. 7.33 above, with two different timings (in dashed and dotted curves). The calculated intensities captured in the cavity due to the three reflectivity functions of Fig. $7.8 \mathrm{p}$ are plotted in Fig.7.8 A. Although the experimental reflectivity function (dashed curve in Fig. 7.8p) differs significantly from the ideal case (full curve) the total power coupled into the cavity is hardly affected (compare the full curve to the dashed curve in Fig. $7.7 \mathrm{k}$ ). The dotted reflectivity curve is delayed relative to the dashed reflectivity curve by half the incident pulse width. Even though this timing error is several times $\tau_{\text {cav }}$ the resulting loss in coupling efficiency is rather modest. This robustness against the exact switching function and timing is a very useful feature for experimental implementation.

\subsection{The variable input coupler}

In the previous, theoretical, section one of the mirrors of the cavity was assumed to have a variable reflectivity. In reality the cavity consists of four highly reflecting mirrors $(R \approx$ 0.9985) and the light is coupled into the cavity using the device depicted in Fig. 7.9. The input coupler consists of a polarizing beam splitter, a Pockels cell (Linos CPC8IM), two half-wave and two quarter-wave plates. In fact, the quarter-wave plates are not essential and are absent in the experiment, but are kept in this section for the sake of clarity. The principle of the input coupler device is as follows: the light circulating in the cavity is horizontally polarized and is transmitted through the beam splitter. The incident light, which is coupled into the cavity, is in phase with the cavity mode, has a vertical polarization and is reflected by the beam splitter. Consequently, the polarization of light just after the beam splitter makes an angle $\theta$ with the horizontal plane. The angle $\theta$ depends on the ratio of the amplitude of the light already inside the cavity and the amplitude of the incident light $\left(A_{\text {cav }}\right.$ and $\left.A_{\text {in }}\right)$. The combination of Pockels cell and retardation plates acts as a rotator for linear polarization. The voltage on the Pockels cell is adjusted continuously to a value which ensures that the polarization at an angle $\theta$ is rotated back into the horizontal plane. Then all the incident light is coupled into the cavity mode with horizontal polarization, no light is coupled back out. At the end, when no more light impinges on the beam splitter, the voltage on the Pockels cell is 0 , the polarization is not rotated anymore and the cavity is therefore closed. 
There is a simple correspondence between the rotation angle $\theta(t)$ and the equivalent reflectivity $R(t)$ of a mirror, used in the theory section; it is given by Eq.7.33 above.

To see that the combination of retardation plates and Pockels cell acts as a polarization rotator, the polarization is written as a two-component vector $(\eta, \xi)$. The components $\eta$ and $\xi$ of the polarization vector are the normalized amplitudes of horizontal and vertical polarization respectively. In this $(\eta, \xi)$ basis, a half-wave plate, oriented such that its optical axis makes an angle of $22.5^{\circ}$ with the horizontal plane, has the matrix representation

$$
\mathbf{H}=\frac{1}{\sqrt{2}}\left(\begin{array}{rr}
1 & 1 \\
1 & -1
\end{array}\right) .
$$

A symmetric phase-shift operator is defined as

$$
\mathbf{P}(\varphi)=\left(\begin{array}{cc}
\exp (i \varphi / 2) & 0 \\
0 & \exp (-i \varphi / 2)
\end{array}\right)
$$

Eqs.7.35 and 7.36 are the usual Jones matrices [216]. The Pockels cell is a variable retarder with retardation angle proportional to the applied voltage. It is now easily verified that the assembly depicted in Fig. 7.9 is indeed a pure polarization rotator:

$$
\mathbf{P}(-\pi / 2) \cdot \mathbf{H} \cdot \mathbf{P}(2 \theta) \cdot \mathbf{H} \cdot \mathbf{P}(\pi / 2)=\left(\begin{array}{cc}
\cos \theta & \sin \theta \\
-\sin \theta & \cos \theta
\end{array}\right),
$$

where $\mathbf{P}( \pm \pi / 2)$ are the quarter-wave plates, and $\mathbf{P}(2 \theta)$ the Pockels cell. The rotation angle $\theta$ of the polarization is half the retardation angle $\varphi$ of the Pockels cell.

The rotation angle is always chosen such that the output polarization is horizontal; the device rotates over an angle equal in size but opposite to that of the linearly polarized light just after the beam splitter.

The optical axes of the two quarter-wave plates are horizontal and vertical respectively. The quarter-wave plates therefore do not mix the cavity and incident polarizations, but just apply to each of them an independent phase shift. In the special case that the output polarization is always horizontal, the second quarter-wave plate has no effect, and can therefore safely be omitted. The first quarter-wave plate can likewise be omitted. Without the first quarter-wave plate there is a constant phase shift of $\pi / 2$ between the two polarization components, from the cavity and incident light. Such a constant phase shift is automatically accounted for by the locking of the cavity. Only the cavity mode which has a phase shift of $\pi / 2$ with the incident light is excited, and this mode has the same properties in a ring cavity than the in-phase mode. The output polarization of the polarization rotator is still linear and horizontal but just after the beam splitter the polarization is now elliptical.

Pulsed dye amplifiers, such as the one used in the experiments in section 7.5, rarely emit Fourier-transform limited pulses. Often a small amount of chirp is present. If this chirp is known and reproducible it can in principle be compensated for. This compensation could be done by changing the cavity length while the pulse enters, using a piezo-driven mirror or by using a second Pockels cell outside of the half-wave plates. Alternatively, one can argue that a partial compensation of the chirp can be obtained without any additional elements by choosing the angle of the optical axes of the two half-wave plates in Fig. 7.9 slightly different from $22.5^{\circ}$. If the angle of the two half-wave plates is $0^{\circ}$ the Pockels cell acts as a pure retarder. At angles of the half-wave plate different than $22.5^{\circ}$ (where a pure 


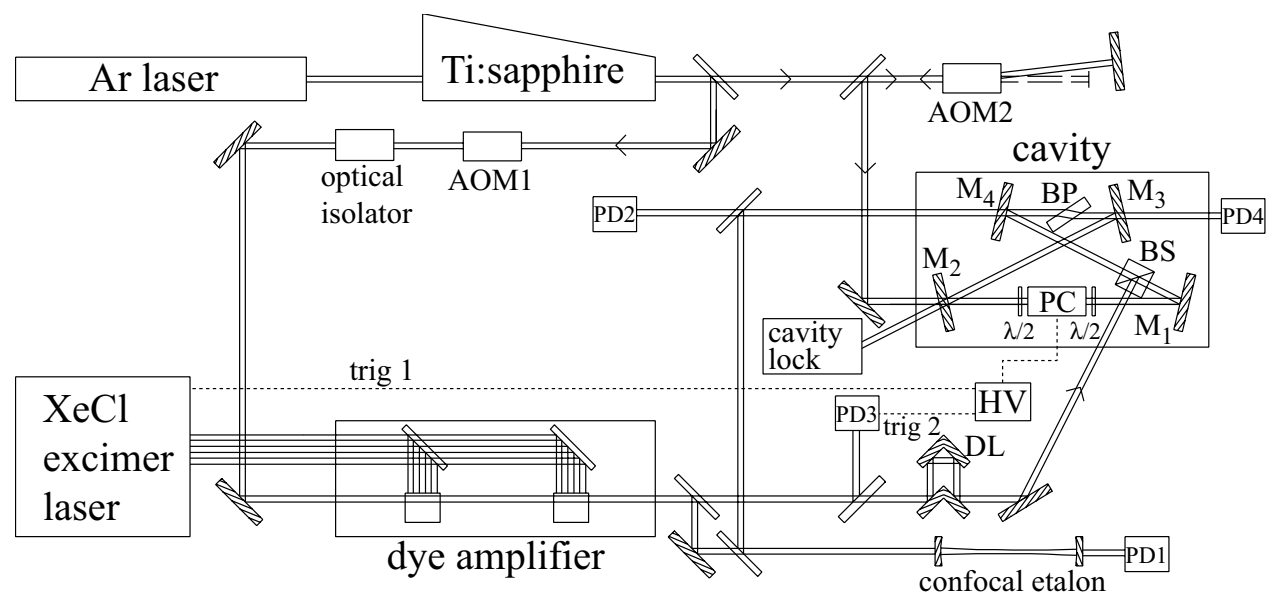

Figure 7.10: Schema of the experimental setup. The simplified cavity of Fig. 7.3 has been modified by replacing the variable input coupler $M_{i}$ by a mirror $M_{1}$, and by adding the Pockels cell-based input coupler of Fig.7.9 instead. The polarizing beam splitter is denoted BS, the Pockels cell PC, the two half-wave plates $\lambda / 2$, and the Brewster plate BP. PD1 through PD4 are photodiodes for diagnostics and control. Adjustment of timing for the high-voltage electronic switch $\mathrm{HV}$ is made with a $7 \mathrm{~m}$ optical delay line DL, and an adjustable length of cable marked trig 2. The electrical triggers are printed in dashed lines. In this figure the lenses and telescopes needed for the mode matching of the signal and reference beams to the cavity are omitted.

polarization rotation takes place) the device in Fig. 7.9 therefore combines the effects of rotation and retardation. The chirp compensation can have both signs, but is not necessarily linear, and depends on the precise form of the time dependence of $\theta$, shown in Eq. 7.34 The analysis of the case with chirp is rather involved and the experiments we performed are inconclusive on this matter. Therefore this chirp compensation is not further pursued in this thesis.

\subsection{Our experimental methods}

In this section the experimental setup and methods chosen for the practical implementation of the switching scheme are described. In Fig. 7.10 the experimental apparatus is shown. The cavity has the geometry of a ring and a length of $0.65 \mathrm{~m}$ corresponding to $\tau_{\text {cav }}=2.2 \mathrm{~ns}$ and $\omega_{\mathrm{FSR}}=460 \mathrm{MHz}$. A cw beam, of $600 \mathrm{~mW}$ at a wavelength of $730 \mathrm{~nm}$, from a titanium:sapphire (Ti:Sa) laser (Coherent 899) is split in two parts. One of the beam from the Ti:Sa laser, the signal beam, is amplified in a pulsed dye amplifier (Lambda Physik FL 2003) pumped by an excimer laser (Lambda Physik LPX210i). The other beam from the Ti:Sa laser, the reference beam, serves to lock the cavity. Note that the combination of Ti:Sa laser and pumped dye amplifier is only one practical way of implementing a pulsed signal beam and cw reference beam at the same frequency, but other (and more table-top) pulsed laser sources can be used. The signal beam provides a pulse of $25 \mathrm{~ns}$ duration and a, tunable, characteristic energy around $50 \mu \mathrm{J}$ to be captured in the cavity. Both signal 
and reference beams are shifted approximately $150 \mathrm{MHz}$ in frequency using acousto-optic modulators (AOM's). A double-pass AOM (AOM2 in Fig. 7.10) is used which allows the frequency of the reference beam to be varied over about $30 \mathrm{MHz}$ without changing the direction of the beam. The use of the tunable AOM allows for compensation of small frequency shifts introduced by the dye amplifier in the signal arm.

One of the cavity mirrors is mounted on a piezo which varies the cavity length in order to lock the cavity to a resonance for the reference beam. To couple the reference beam into the cavity, mirror $M_{2}$ has a transmission coefficient of $0.5 \%$. The variable input coupler described in the previous section is embedded inside the cavity. The Pockels cell is normally switched off and hence the input coupling device is transparent apart from small absorption and reflection losses. The combined losses of the cavity mirrors and the intra-cavity elements limit the finesse of the cavity to about 60 . This finesse corresponds to roughly $10 \%$ loss or a ring-down time $\tau_{\mathrm{r}} \approx 10 \tau_{\text {cav }}$. These comparatively high losses are mainly due to non-perfect components, particularly the Pockels cell, polarizing beam splitter and waveplates.

The pulse from the dye amplifier is captured into the cavity using the following procedure: the voltage on the Pockels cell is switched from 0 to $4 \mathrm{kV}$ about $1 \mu$ s before the firing of the excimer laser (trig1 in Fig. 7.10). The $4 \mathrm{kV}$ voltage corresponds to a retardation of the Pockels cell of approximately $\pi$ and therefore to a $\pi / 2$ polarization rotation, i.e., a fully open cavity (see section 7.4). A small part of the incident pulse is picked off and led to a fast photodiode (PD3) to serve as a trigger for a fast high-voltage switch. This voltage switch, which is described in detail below, brings back the voltage on the Pockels cell from 4 to $0 \mathrm{kV}$ in a precisely timed fashion in approximately $10 \mathrm{~ns}$ : about half of the pulse duration. The timing of the second trigger signal relative to the arrival time of the amplified pulse at the polarizing beam splitter in the cavity is determined by a variable cable length (trig2 in Fig. 7.10) combined with an optical delay line of about $7 \mathrm{~m}$.

The reference beam traverses the cavity in the opposite direction than the pulsed signal beam. When the Pockels cell is switched off the cavity is locked to one of its resonances for the reference beam using the Hänsch-Couillaud method [217]. Since the phase of the cavity mode is set by the input beam, in such a way that after one round-trip the cavity mode and the input beam interfere constructively, any necessary stationary phase difference between the two beams is automatically taken care of by the locking. The time constant of the locking scheme is of the order of $100 \mu \mathrm{s}$. The Pockels cell is briefly switched on and off for about $1 \mu$ s to capture the pulse. The slow dynamics of the locking ensures that the cavity stays at resonance during the necessary time for the capture. The astigmatism of the cavity resulting from the two curved mirrors $M_{3}$ and $M_{4}$ is compensated [218] by a 1-cm-thick BK7 plate at Brewster angle. This Brewster plate can be replaced by a frequency-doubling crystal of similar length and refractive index. The pulsed amplification scheme introduces a frequency shift of typically $20 \mathrm{MHz}$ in the output of the signal beam. This frequency shift varies and increases gradually with aging dye. To ensure that the center frequency of the signal coincides with the reference frequency, the spectrum of both beams are compared using a confocal etalon with $\omega_{\mathrm{FSR}}=150 \mathrm{MHz}$ and $F=100$, and the difference is compensated using the double-pass AOM. The length of the confocal etalon is fixed. In order to measure a power spectrum by using this etalon, the frequency of the Ti:Sa laser is slowly scanned. The amplified pulses are sent through the etalon simultaneously with the refer- 
Figure 7.11: Schematics of the high-voltage switching electronics for the Pockels cell. The triggers trig. 1 and trig. 2 are TTL level signals for on and off switching. trig.1 also triggers the excimer laser and trig. 2 is taken directly from the fast photodiode PD3 measuring the amplified pulse.

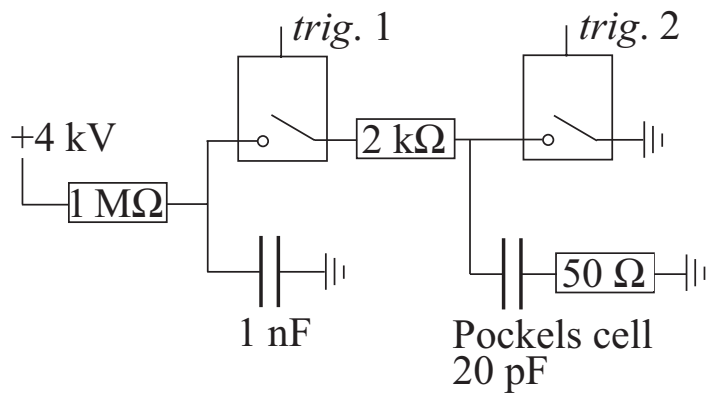

ence beam. The pulsed light is detected using 'sample and hold' electronics synchronized with the firing of the excimer laser. The scan time over one free spectral range of the etalon is about $10 \mathrm{~s}$. The long scan time ensures sufficient spectrum resolution when firing the excimer laser at $10 \mathrm{~Hz}$ or higher.

The confocal etalon is also used to measure the spectrum of the light that is stored in the cavity. The leakage through one of the high-reflecting cavity mirrors $\left(M_{4}\right.$ in Fig. 7.10 is led to the etalon. Only about $10^{-4}$ of the intensity of light present in the cavity leaks out through $M_{4}$ but this leakage is sufficient to perform a frequency measurement through the etalon. Again the frequency of the Ti:Sa laser is scanned, affecting both reference and signal beams in the same way. The cavity lock follows the scan of the reference beam frequency and hence the cavity also stays at resonance for the amplified pulsed signal.

The last part of the experimental setup is the implementation of the voltage switching of the Pockels cell, illustrated in Fig. 7.11. The switching is done by combining two fast solidstate high-voltage switches. Both switches act as effective short-circuit upon a TTL trigger. The first one, trig.1 (Behlke HTS50-06) switches the voltage across the Pockels cell from 0 to $4 \mathrm{kV}$. The switching trig. 1 and the excimer laser are triggered simultaneously. The actual firing of the excimer laser is delayed with respect to this trigger by about $1.3 \mu$ s with a pulse to pulse fluctuation of $0.2 \mu \mathrm{s}$. When the excimer laser is fired, the optical signal is used to trigger a second fast switch (Alphalas HVS 4000-F) which short-circuits the Pockels cell. This second switch has an almost instantaneous (within a few nanoseconds) and practically jitter-free response. The second switch, being driven by a TTL level trigger, is rather sensitive to high-voltage cross talk from the first switch and initially operated at unwanted moments. This problem was solved by empirically adding resistors and parallel capacitors. These extra electrical components also served to set the time constant, with which the voltage across the Pockels cell drops, to the desired value of $10 \mathrm{~ns}$, about half the pulse duration. The additional circuit elements have been omitted in Fig. 7.11 for simplicity. The voltage on the Pockels cell is an exponential decrease from 4 to $0 \mathrm{kV}$ with a time constant $\tau_{\mathrm{s}}$ of typically $10 \mathrm{~ns}$. The resulting effective reflectivity is obtained using Eq. 7.33 and is depicted as the dashed curve in Fig. 7.8p. 


\subsection{Experimental results}

To experimentally corroborate the ideas outlined in the first sections of this chapter, two types of experiments were performed: in the time domain and in the frequency domain. The light present inside the cavity can be monitored as a function of time by observing the leakage light through $M_{4}$ on a fast photodiode (PD2). Alternatively, the power spectrum of this leakage light can be obtained by guiding it through the confocal etalon, integrating the pulse and detecting its magnitude as a function of the laser frequency.

The relative frequency of the reference and signal beams and the timing of triggering of the second high voltage switch in Fig. 7.11 are empirically varied until the light in the cavity has its power maximized and its bandwidth minimized. Introducing a deviation from $22.5^{\circ}$ of the angle of the half-wave plates relative to the horizontal direction to compensate a possible chirp had little effect. Consequently all measurements were taken at the original orientation of these wave plates. Once the optimal setting was found the situation was stable and the pulse-to-pulse reproducibility proved sufficient to ensure a reliable operation.

In Fig. $7.12 \mathrm{a}$ and $7.12 \mathrm{p}$ the intensity of light leaking through $M_{4}$ is shown in the time and frequency domain, respectively. The pulse stored in the switched cavity is compared to the pulse as it is admitted. The latter is measured with an open input coupler (Pockels cell at constant $V=4 \mathrm{kV}$ ) and the cavity blocked so the light can not complete the first round-trip. The blocked-cavity signal has exactly the temporal profile of the pulse from the amplifier. The amplitude of the blocked-cavity signal is smaller because the beam splitter rejects a part of the incoming beam, some additional losses in the cavity further reduces it and only the small leakage through $M_{4}$ is measured. By taking the leakage light from the blocked cavity as an effective definition of the input pulse, the effect of rejection by the polarizing cube and the leakage ratio are eliminated, and the amplifying effect of the cavity can be directly determined. As can be seen in Fig. 7.12 a the signal in the cavity (2) becomes approximately a factor 10 bigger than the incident pulse (1) if the cavity is switched, and optimized for maximum signal. In Fig. 7.12 the signal (3) leaking from a cavity with a fixed reflectivity $R=50 \%$ (i.e., a fixed voltage on the Pockels cell $V=2 \mathrm{kV}$ ) is also shown. For fixed input coupling this choice of reflectivity couples the maximum energy into the cavity. From Fig. 7.12 the pulse is found to be coupled into the switched cavity with about $90 \%$ efficiency. Indeed, within our definition of the input pulse, the peak intensity in transmission is characteristic of the intensity inside the cavity. The incident energy is the integral of the incident pulse. The energy in the cavity is the intensity in the cavity during one round-trip. The efficiency of the pulse capture is the ratio of the peak intensity in the switched case multiplied by $\tau_{\text {cav }}$ and the integral of the incident pulse.

In Fig. 7.12 p the power spectrum of the captured pulse is shown. Four cases are compared: the power spectrum of the pulsed light before it enters the cavity (1), the spectrum after the switched cavity (2), and two spectra for a cavity with fixed effective reflectivity $(3,4): R=95 \%(V=0.3 \mathrm{kV})$ and $68 \%(V=1.5 \mathrm{kV})$, respectively. The three spectra measured after the light has passed the cavity are taken in identical way using the light leaking through mirror $M_{4}$ and their amplitude can be directly compared. The spectrum of the pulse before it enters the cavity is measured directly and is consequently more intense. The spectrum of the incident pulse has been scaled in Fig.7.12p to a size convenient for comparing its spectral width to the other spectra. In the case of a large fixed $R$ the bandwidth of the 


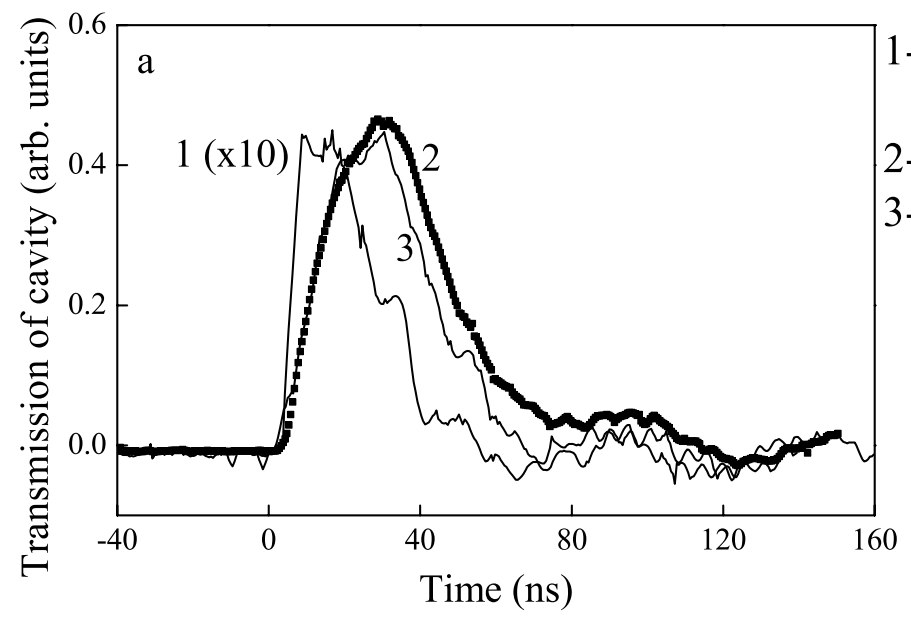

1- Incident pulse (x10)

2- Switched cavity

3 - fixed $\mathrm{R}=50 \%$

$(V=2 \mathrm{kV})$

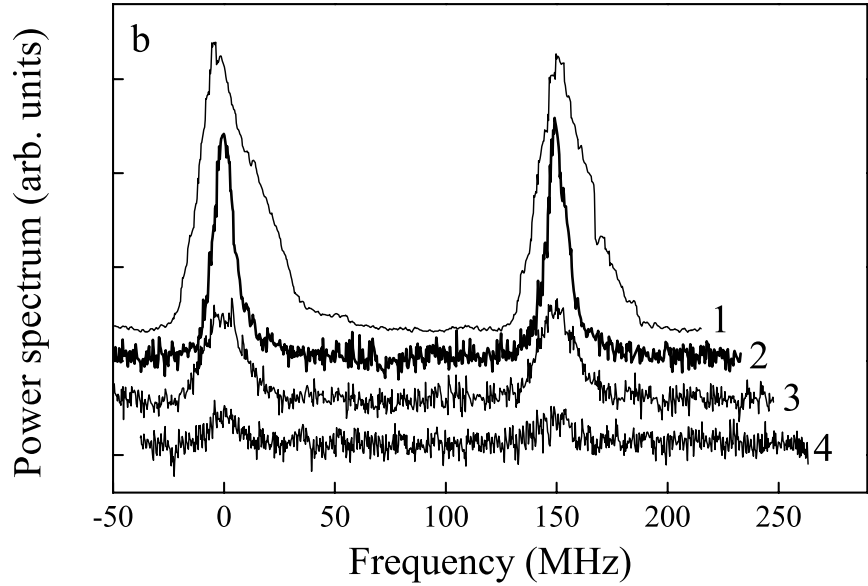

1- Incident pulse $\delta \omega=25.2 \pm 2.0 \mathrm{MHz}$

2- Switched cavity $\delta \omega=10.0 \pm 0.5 \mathrm{MHz}$

3 - fixed $\mathrm{R}=68 \%$

$(V=1.5 \mathrm{kV})$ $\delta \omega=17.9 \pm 0.5 \mathrm{MHz}$

4- fixed $\mathrm{R}=95 \%$

$(V=0.3 \mathrm{kV})$

$\delta \omega=10.9 \pm 1.5 \mathrm{MHz}$

Figure 7.12: Characteristics of a long light pulse captured in a cavity. In (a), the leakage light of the captured pulse in the cavity (2) is shown versus time. The time trace obtained from the incident pulse multiplied by 10 (1) and a trace for a stationary cavity (3) with $R=50 \%$ are shown for comparison. In (b) the power spectrum of the incident pulse (1) is compared to that obtained for a switched cavity (2) and a cavity with two different, constant, effective reflectivities $(3,4)$ of the input coupler. The switched cavity clearly captures both a higher power and narrower bandwidth than the cavity with fixed reflectivity. The spectral width $\delta \omega$ of each spectrum is given for quantitative comparison. The scale of the lower three spectra $(2,3,4)$ can be directly compared. The reference spectrum (1) is measured before the cavity, therefore its scale can not be compared. The zero of the frequency scale is set arbitrarily at the position of the left transmission peak. 
light is small but so is the overall signal. This high-reflectivity case corresponds to filtering out all but the resonant frequency components of the pulse. For smaller but still fixed $R$ the captured power is bigger but so is the bandwidth. In the switched case, both the maximum bandwidth reduction and the largest signal is obtained, when the whole pulse is captured.

When comparing the switched case to that of fixed input coupling it is useful to consider the ratio power/bandwidth as a figure of merit. For fixed voltage the figure of merit shows a broad maximum in the range 1.5-2 kV. The power spectra such as those shown in Fig. $7.12 \mathrm{p}$ indicate an improvement of about a factor 2 in this figure of merit when comparing the switched case to the best fixed input case.

In principle the figure of merit of the previous paragraph should carry over to the time domain, in other words the time trace for the switched case is expected to have a longer tail. However, the results of Fig. $7.12 \mathrm{a}$ in the time domain indicate a small difference between the switched case and the optimal choice of fixed reflectivity. This apparent discrepancy between time and frequency domain can be understood by realizing that the experimental pulse is not Fourier-transform limited. The duration of the incident pulse is larger than that resulting from the Fourier transform of the spectrum, making the time-domain signals more sensitive to the rather large losses of the cavity. By making a comparison to the theoretical predictions for the case of $10 \%$ loss, for the switched case in Fig. 7.7 a and for $R=80 \%$ in Fig. 7.7p the difference is found to be approximately as small as in the experiment, for comparable parameters.

In Fig. 7.13 the experimental results are summarized in a more quantitative way. It can be seen from Fig. 7.13 that the integral of the power spectrum and hence the total power coupled into the cavity, in the fixed-voltage case, goes through a maximum at about $R=50 \%(V=2 \mathrm{kV})$ but is always below the power in the switched case. The bandwidth, in Fig. 7.13 a, on the other hand is always bigger for a fixed reflectivity than in the switched case, approaching it only for large reflectivity $(V \rightarrow 0)$, as expected. The theoretical curve shown in Fig. 7.13 is from Eqs. 7.15 and 7.17 with the measured pulse spectrum approximated by a Lorentzian. The expected bandwidth in the cavity depends on only two parameters: the spectral width $\Gamma=26 \mathrm{MHz}$ of the incident pulse and the cavity loss rate of $10 \%$ ( or $\alpha^{2}=0.9$ ). Both are determined experimentally in independent measurements. The incident bandwidth $\Gamma$ is measured explicitly and $\alpha^{2}$ follows from the measured cavity finesse using the reference beam. The data in Fig. 7.13 have been deconvoluted with the instrumental response of the confocal etalon which adds about $1.5 \mathrm{MHz}$. The good agreement between theory and experiment in Fig. 7.13 gives confidence in the analysis. The total power versus voltage is slightly harder to analyze because it depends more critically on pulse shape and spectral properties. In Fig. $7.13 \mathrm{~b}$ the experimental results are compared to a numerical calculation for a Gaussian pulse with the same parameters as in Figs. 7.7 and 7.8. The experimentally observed maximum occurs at a somewhat different value than in the theoretical prediction but the height of the maximum compared to the switched case is similar. It is likely that the quantitative differences between experiments and theory are due to the fact that the experimental pulse is not Gaussian, and not transform limited. 

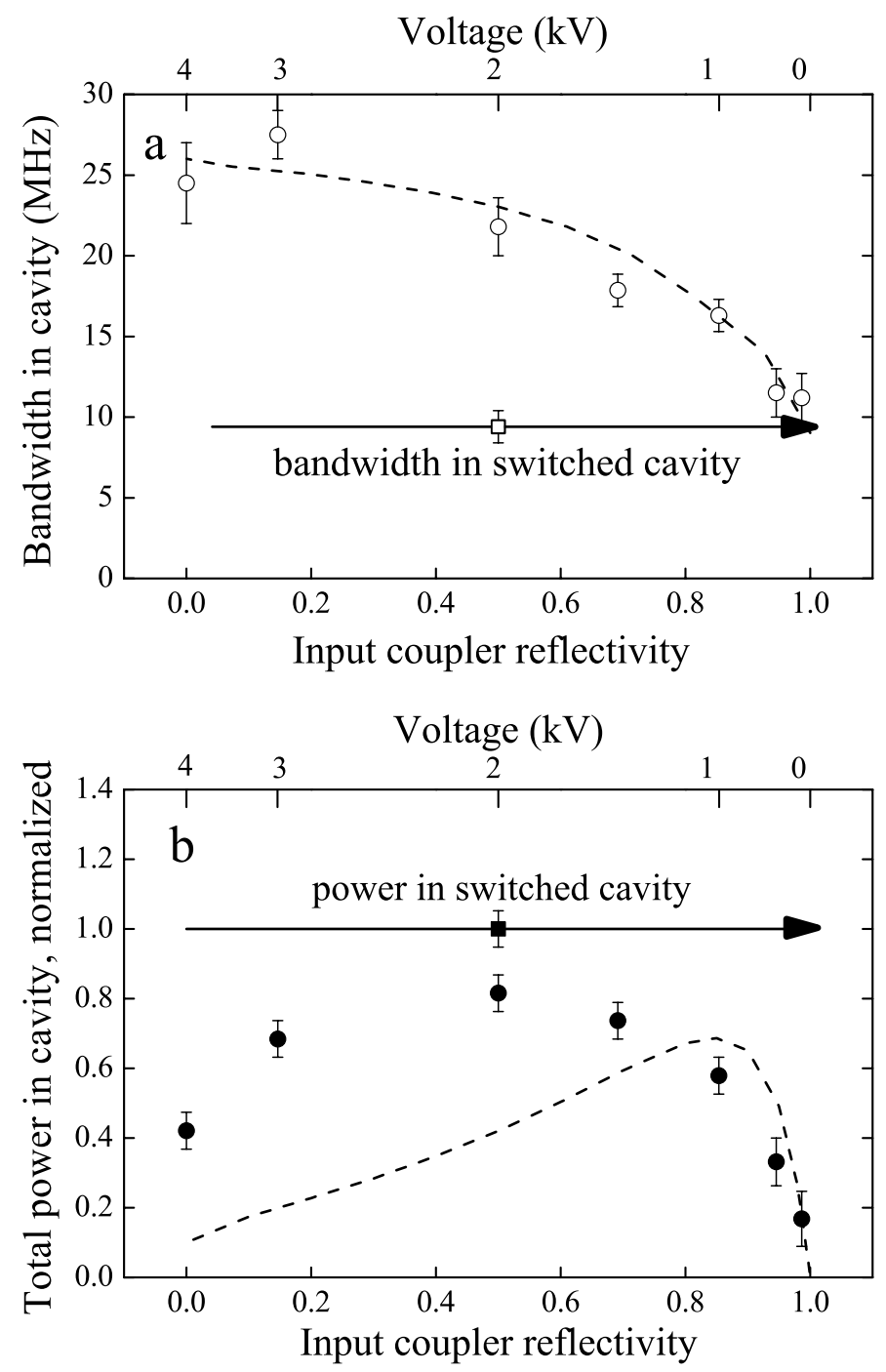

Figure 7.13: Summary of experimental results. In (a) the width of the power spectrum is shown versus fixed reflectivity of the input coupler (open circles) and compared to the switched case (open square). The arrow through the square indicates that the reflectivity is swept from 0 to 1 as the pulse is admitted. The top axis gives the corresponding voltage on the Pockels cell. The dotted curve is the theory for the fixed reflectivity case. In (b) the total power inside the cavity, or integral of the power spectrum, normalized to the switched case, is shown versus the input coupler reflectivity (filled circles). The filled square corresponds to the switched case. The dotted curve is a numerical calculation. Capturing a pulse in a cavity allows the smallest bandwidth simultaneously with the highest power to be obtained compared to a stationary cavity. 


\subsection{Conclusions}

A scheme to combine high power and narrow bandwidth was proposed. A light pulse can be fully captured in short cavity of high finesse, provided the input reflectivity of the cavity is matched to the shape of the incident pulse. The pulse capture is very robust against variations from the optimal input reflectivity function. Experimentally, the implementation of this scheme was done by switching the voltage across a Pockels cell, effectively varying the coupling of the polarized incident beam with the cavity mode of crossed polarization. The capture of a light pulse in a short high-finesse cavity has been accomplished. A light pulse has been compressed in the frequency domain and at the same time its peak intensity increased. The present experiment can be seen as a proof of principle of the scheme. There is considerable room for improvement through careful selection of dedicated quality components resulting in smaller losses. We believe that in order to realize reliable applications an on-chip or integrated implementation using fiber optics is desirable as well as practically viable. An improvement on the quality of the scheme implementation would also open the way to a useful application in reversible capture and release experiments. 


\section{References}

[1] J. W. S. Rayleigh, On the light from the sky, its polarization and colour, Philos. Mag. 41, 107, 274 (1871).

[2] J. W. S. Rayleigh, On the scattering of light by small particles, Philos. Mag. 41, 447 (1871).

[3] C. F. Bohren and D. R. Huffman, Absorption and scattering of light by small particles (John Wiley \& Sons, New York, 1983).

[4] H. C. van de Hulst, Light scattering by small particles (Dover, New York, 1981).

[5] G. Mie, Beitrage zur Optik truber Medien, speziell kolloidaler Metallosungen, Ann. Phys. (Leipzig) 25, 377 (1908).

[6] Y.-L. Geng, X.-B. Wu, L.-W. Li, and B. R. Guan, Mie scattering by a uniaxial anisotropic sphere, Phys. Rev. E 70, 056609 (2004).

[7] H. S. Carslaw and J. C. Jaeger, Conduction of heat in solids, 2nd ed. (Oxford University Press, Oxford, 1959).

[8] J. J. Duderstadt and L. J. Hamilton, Nuclear reactor analysis (John Wiley \& Sons, New York, 1976).

[9] S. Glasstone and M. C. Edlund, The elements of nuclear reactor theory (Van Nostrand, Princeton, 1952).

[10] H. This, Les secrets de la casserole (Belin, Tours, 1993).

[11] M. B. van der Mark, Propagation of light in disordered media: a search for Anderson localization, Ph.D. thesis, University of Amsterdam, 1990, http://www.wavesincomplexmedia.com.

[12] B. Shapiro, Large intensity fluctuations for wave propagation in random media, Phys. Rev. Lett. 57, 2168 (1986).

[13] B. A. van Tiggelen, P. Sebbah, M. Stoytchev, and A. Z. Genack, Delay-time statistics for diffusive waves, Phys. Rev. E 59, 7166 (1999).

[14] P. Sebbah, O. Legrand, and A. Z. Genack, Fluctuations in photon local delay time and their relation to phase spectra in random media, Phys. Rev. E 59, 2406 (1999).

[15] A. Z. Genack, P. Sebbah, M. Stoytchev, and B. A. van Tiggelen, Statistics of wave dynamics in random media, Phys. Rev. Lett. 82, 715 (1999).

[16] F. Bardou, J.-P. Bouchaud, A. Aspect, and C. Cohen-Tannoudji, Lévy statistics and laser cooling (Cambridge University Press, Cambridge, 2002).

[17] A. A. Golubentsev, Suppression of interference effects in multiple scattering of light, Zh. Éksp. Teor. Fiz. 86, 47 (1984), [Sov. Phys. JETP 59, 26 (1984)].

[18] B. L. Altshuller, A. G. Aronov, and D. E. Khmelnitsky, Suppression of localization effects by the high-frequency field and the Nyquist noise, Solid State Commun. 39, 619 (1981).

[19] P. W. Anderson, Absence of diffusion in certain random lattices, Phys. Rev. 109, 1492 (1958).

[20] S. John, Electromagnetic absorption in a disordered medium near a photon mobility edge, Phys. Rev. Lett. 53, 2169 (1984).

[21] P. W. Anderson, The question of classical localization. A theory of white paint?, Philos. Mag. 
B 55, 2692 (1985).

[22] E. Abrahams, P. W. Anderson, D. C. Licciardello, and T. V. Ramakrishnan, Scaling theory of localization: absence of quantum diffusion in two dimensions, Phys. Rev. Lett. 42, 673 (1979).

[23] P. Sheng, Introduction to wave scattering, localization, and mesoscopic phenomena (Academic Press, New York, 1995).

[24] A. F. Ioffe and A. R. Regel, Non-crystalline, amorphous, and liquid electronic semiconductors, Prog. Semicond. 4, 237 (1960).

[25] D. Vollhardt and P. Wölfle, Scaling equations from a self-consistent theory of Anderson localization, Phys. Rev. Lett. 48, 699 (1982).

[26] E. N. Economou, C. M. Soukoulis, and A. D. Zdetsis, Conductivity in disordered systems, Phys. Rev. B 31, 6483 (1985).

[27] N. Garcia and A. Z. Genack, Anomalous photon diffusion at the threshold of the Anderson localization transition, Phys. Rev. Lett. 66, 1850 (1991).

[28] P. M. Johnson, A. Imhof, B. P. J. Bret, J. Gómez Rivas, and A. Lagendijk, Pulse propagation in a strongly scattering material, Phys. Rev. E 68, 016604 (2003).

[29] M. Stoytchev and A. Z. Genack, Observations of non-Rayleigh statistics in the approach to photon localization, Opt. Lett. 24, 262 (1999).

[30] A. A. Chabanov, M. Stoytchev, and A. Z. Genack, Statistical signatures of photon localization, Nature (London) 404, 850 (2000).

[31] E. Yablonovitch, Inhibited spontaneous emission in solid-state physics and electronics, Phys. Rev. Lett. 58, 2059 (1987).

[32] S. John, Strong localization of photons in certain disordered dielectric superlattices, Phys. Rev. Lett. 58, 2486 (1987).

[33] N. W. Ashcroft and N. D. Mermin, Solid state physics (Holt, Rinehard and Winston, New York, 1976).

[34] J. D. Joannopoulos, R. D. Meade, and J. N. Winn, Photonic crystals: molding the flow of light (Princeton University Press, Princeton, 1995).

[35] P. M. Johnson, A. F. Koenderink, and W. L. Vos, Ultrafast switching of photonic density of states in photonic crystals, Phys. Rev. B 66, 081102 (2002).

[36] T. G. Euser and W. L. Vos, Spatial homogeneity of optically switched semiconductor photonic crystals and of bulk semiconductors, J. Appl. Phys. 97, 043102 (2005).

[37] A. F. Koenderink, L. Bechger, H. P. Schriemer, A. Lagendijk, and W. L. Vos, Broadband fivefold reduction of vacuum fluctuations probed by dyes in photonic crystals, Phys. Rev. Lett. 88, 143903 (2002).

[38] P. Lodahl, A. F. van Driel, I. S. Nikolaev, A. Irman, K. Overgaag, D. Vanmaekelbergh, and W. L. Vos, Controlling the dynamics of spontaneous emission from quantum dots by photonic crystals, Nature (London) 430, 654 (2004).

[39] J. E. G. J. Wijnhoven and W. L. Vos, Preparation of photonic crystals made of air spheres in titania, Science 281, 802 (1998).

[40] J. E. G. J. Wijnhoven, L. Bechger, and W. L. Vos, Fabrication and characterization of large macroporous photonic crystals in titania, Chem. Mater. 13, 4486 (2001).

[41] E. Yablonovitch, T. J. Gmitter, R. D. Meade, A. M. Rappe, K. D. Brommer, and J. D. Joannopoulos, Donor and acceptor modes in photonic band structure, Phys. Rev. Lett. 67, 3380 (1991).

[42] P. R. Villeneuve, S. Fan, and J. D. Joannopoulos, Microcavities in photonic crystals: mode symmetry, tunability, and coupling efficiency, Phys. Rev. B 54, 7837 (1996).

[43] K. Sakoda, Optical properties of photonic crystals (Springer Verlag, Berlin, 2001).

[44] A. F. Koenderink, M. Megens, G. van Soest, W. L. Vos, and A. Lagendijk, Enhanced backscat- 
tering from photonic crystals, Phys. Rev. A 268, 104 (2000).

[45] A. F. Koenderink and W. L. Vos, Light exiting from real photonic band gap crystals is diffuse and strongly directional, Phys. Rev. Lett. 91, 213902 (2003).

[46] W. Gellermann, M. Kohmoto, B. Sutherland, and P. C. Taylor, Localization of light waves in Fibonacci dielectric multilayers, Phys. Rev. Lett. 72, 633 (1994).

[47] T. Hattori, N. Tsurumachi, S. Kawato, and H. Nakatsuka, Photonic dispersion relation in a one-dimensional quasicrystal, Opt. Commun. 35, 441 (1994).

[48] L. Dal Negro, C. J. Oton, Z. Gaburro, L. Pavesi, P. M. Johnson, A. Lagendijk, R. Righini, M. Colocci, and D. S. Wiersma, Light transport through the band-edge states of fibonacci quasicrystals, Phys. Rev. Lett. 90, 055501 (2003).

[49] Photonic crystals and light localization in the $21^{\text {st }}$ century, edited by C. M. Soukoulis (Kluwer, Dordrecht, 2001).

[50] Waves scattering in complex media: from theory to applications, edited by B. A. van Tiggelen and S. E. Skipetrov (Kluwer, Dordrecht, 2003).

[51] E. Akkermans and G. Montambaux, Physique mésoscopique des électrons et des photons (EDP Sciences, Les Ulis, 2004).

[52] J. L. Thomas and M. Fink, Ultrasonic beam focusing through tissue inhomogeneities with a time reversal mirror: application to transskull therapy, IEEE Trans. Ultrason. Ferroelec. Freq. Contr. 43, 1122 (1996).

[53] R. H. J. Kop and R. Sprik, Phase-sensitive interferometry with ultrashort optical pulses, Rev. Sci. Instrum. 66, 5459 (1995).

[54] M. Fink, Time reversed acoustics, Phys. Today March, 34 (1997).

[55] J. L. Thomas, P. Roux, and M. Fink, Inverse scattering analysis with an acoustic time-reversal mirror, Phys. Rev. Lett. 72, 637 (1994).

[56] A. Derode, P. Roux, and M. Fink, Robust acoustic time reversal with high-order multiple scattering, Phys. Rev. Lett. 75, 4206 (1995).

[57] R. Pappu, B. Recht, J. Taylor, and N. Gershenfeld, Physical one-way functions, Science 297, 2026 (2002).

[58] A. L. Moustakas, H. U. Baranger, L. Balents, A. M. Sengupta, and S. H. Simon, Communication through a diffusive medium: coherence and capacity, Science 287, 287 (2000).

[59] A. Derode, A. Tourin, J. de Rosny, M. Tanter, S. Yon, and M. Fink, Taking advantage of multiple scattering to communicate with time-reversal antennas, Phys. Rev. Lett. 90, 014301 (2003).

[60] V. S. Letokhov, , Zh. Eksp. Teor. Fiz. 53, 1442 (1968), [Sov. Phys. JETP 26, 835].

[61] C. Gouedard, D. Husson, C. Sauteret, F. Auzel, and A. Migus, Generation of spatially incoherent short pulses in laser-pumped neodymium stoichiometric crystals and powders, J. Opt. Soc. Am. B 10, 2358 (1993).

[62] H. Cao, J. Y. Xu, E. W. Seelig, and R. P. H. Chang, Microlaser made of disordered media, Appl. Phys. Lett. 76, 2997 (2000).

[63] H. Cao, J. Y. Xu, D. Z. Zhang, S.-H. Chang, S. T. Ho, E. W. Seelig, X. Liu, and R. P. H. Chang, Spatial confinement of laser light in active random media, Phys. Rev. Lett. 84, 5584 (2000).

[64] V. Milner and A. Z. Genack, Photon localization laser: low-threshold lasing in a random amplifying layered medium via wave localization, Phys. Rev. Lett. 94, 073901 (2005).

[65] P. Lodahl and A. Lagendijk, Transport of quantum noise through random media, Phys. Rev. Lett. 94, 153905 (2005).

[66] P. Lodahl, A. P. Mosk, and A. Lagendijk, Spatial quantum correlations in multiple scattered light, (2005), available at http://arxiv.org/abs/quant-ph/0502033.

[67] L. A. Golovan', V. A. Mel'nikov, S. O. Konorov, A. B. Fedotov, S. A. Gavrilov, A. M. 
Zheltikov, P. K. Kashkarov, V. Y. Timoshenko, G. I. Petrov, L. Li, and V. V. Yakovlev, Efficient second-harmonic generation by scattering from porous gallium phosphide, JETP Lett 78, 193 (2003).

[68] V. A. Mel'nikov, L. A. Golovan', S. O. Konorov, D. A. Muzychenko, A. B. Fedotov, A. M. Zheltikov, V. Y. Timoshenko, and P. K. Kashkarov, Second-harmonic generation in strongly scattering porous gallium phosphide, Appl. Phys. B 79, 225 (2004).

[69] M. Baudrier-Raybaut, R. Haïdar, P. Kupecek, P. Lemasson, and E. Rosencher, Random quasiphase-matching in bulk polycrystalline isotropic nonlinear materials, Nature (London) 432, 374 (2004).

[70] S. E. Skipetrov, Disorder is the new order, Nature (London) 432, 285 (2004).

[71] I. M. Tiginyanu, I. V. Kravetsky, J. Monecke, W. Cordts, G. Marowsky, and H. L. Hartnagel, Semiconductor sieves as nonlinear optical materials, Appl. Phys. Lett. 77, 2415 (2000).

[72] J. M. Drake and A. Z. Genack, Observation of non-classical optical diffusion, Phys. Rev. Lett. 63, 259 (1989).

[73] R. H. J. Kop, P. de Vries, R. Sprik, and A. Lagendijk, Observation of anomalous transport of strongly multiple scattered light in thin disordered slabs, Phys. Rev. Lett. 79, 4369 (1997).

[74] H. Cao, Y. G. Zhao, H. C. Ong, S. T. Ho, J. Y. Dai, J. Y. Wu, and R. P. H. Chang, Ultraviolet lasing in resonators formed by scattering in semiconductor polycrystalline films, Appl. Phys. Lett. 73, 3656 (1998).

[75] G. van Soest, Experiments on random lasers, Ph.D. thesis, University of Amsterdam, 2001, $\mathrm{http}: / / \mathrm{www}$. wavesincomplexmedia.com.

[76] D. S. Wiersma, P. Bartolini, A. Lagendijk, and R. Righini, Localization of light in a disordered medium, Nature (London) 390, 671 (1997), see also [138, 179].

[77] J. Gómez Rivas, R. Sprik, and A. Lagendijk, Optical transmission through very strong scattering media, Ann. Phys. (Leipzig) 8, 77 (1999).

[78] J. Gómez Rivas, R. Sprik, A. Lagendijk, L. C. Noordam, and C. W. Rella, Midinfrared scattering and absorption in Ge powder close to the Anderson localization transition, Phys. Rev. E 62, R4540 (2000).

[79] J. Gómez Rivas, R. Sprik, A. Lagendijk, L. C. Noordam, and C. W. Rella, Static and dynamic transport of light close to the Anderson localization transition, Phys. Rev. E 63, 046613 (2001).

[80] J. Gómez Rivas, R. Sprik, C. M. Soukoulis, K. Busch, and A. Lagendijk, Optical transmission through strong scattering and highly poydisperse media, Europhys. Lett. 48, 22 (1999).

[81] J. Gómez Rivas, Light in strongly scattering semiconductors - diffuse transport and Anderson localization, Ph.D. thesis, University of Amsterdam, 2002, http://www.wavesincomplexmedia.com.

[82] F. J. P. Schuurmans, D. Vanmaekelbergh, J. van de Lagemaat, and A. Lagendijk, Strongly photonic macroporous gallium phosphide networks, Science 284, 141 (1999).

[83] F. J. P. Schuurmans, M. Megens, D. Vanmaekelbergh, and A. Lagendijk, Light scattering near the localization transition in macroporous GaP networks, Phys. Rev. Lett. 83, 2183 (1999).

[84] J. Gómez Rivas, R. W. Tjerkstra, D. Vanmaekelbergh, J. J. Kelly, and A. Lagendijk, Tunable photonic strength in porous GaP, Appl. Phys. Lett. 80, 4498 (2002).

[85] B. H. Erné, D. Vanmaekelbergh, and J. J. Kelly, Porous etching - a means to enhance the photoresponse of indirect semiconductors, Adv. Mater. 7, 739 (1995).

[86] B. H. Erné, High quantum yield III-V photoanodes, Ph.D. thesis, University of Utrecht, 1995.

[87] B. H. Erné, D. Vanmaekelbergh, and J. J. Kelly, Morphology and strongly enhanced photoresponse of GaP electrodes made porous by anodic etching, J. Electrochem. Soc. 143, 305 (1996).

[88] W. Xue, P. Sheng, Q.-J. Chu, and Z.-Q. Zhang, Localization transition in media with 
anisotropic diagonal disorder, Phys. Rev. Lett. 63, 2837 (1989).

[89] Z.-Q. Zhang, Q.-J. Chu, W. Xue, and P. Sheng, Anderson localization in anisotropic random media, Phys. Rev. B 42, 4613 (1990).

[90] P. M. Johnson, B. P. J. Bret, J. Gómez Rivas, J. J. Kelly, and A. Lagendijk, Anisotropic diffusion of light in a strongly scattering material, Phys. Rev. Lett. 89, 243901 (2002).

[91] A. F. van Driel, B. P. J. Bret, D. Vanmaekelbergh, and J. J. Kelly, Hot carrier luminescence during porous etching of GaP under high electric field conditions, Surf. Sci. 529, 197 (2003).

[92] B. P. J. Bret, T. L. Sonnemans, and T. W. Hijmans, Capturing a light pulse in a short highfinesse cavity, Phys. Rev. A 68, 023807 (2003).

[93] J. Gómez Rivas, D. H. Dau, A. Imhof, R. Sprik, B. P. J. Bret, P. M. Johnson, T. W. Hijmans, and A. Lagendijk, Experimental determination of the effective refractive index in strongly scattering media, Opt. Commun. 220, 17 (2003).

[94] B. P. J. Bret and A. Lagendijk, Anisotropic enhanced backscattering induced by anisotropic diffusion, Phys. Rev. E 70, 036601 (2004).

[95] A. Lagendijk and B. A. van Tiggelen, Resonant multiple scattering of light, Phys. Rep. 270, 143 (1996).

[96] M. B. van der Mark, M. P. van Albada, and A. Lagendijk, Light scattering in strongly scattering media: multiple scattering and weak localization, Phys. Rev. B 37, 3575 (1988).

[97] P. N. den Outer, Multiple light scattering in random scattering media, Ph.D. thesis, University of Amsterdam, 1995, http://www.wavesincomplexmedia.com.

[98] A. Lagendijk, R. Vreeker, and P. de Vries, Influence of internal reflection on diffusive transport in strongly scattering media, Phys. Lett. A 136, 81 (1989).

[99] P. M. Morse and H. Feshbach, Methods of theoretical physics (McGraw-Hill, New York, 1953).

[100] E. N. Economou, Green's function in quantum physics (Springer Verlag, Heidelberg, 1979).

[101] B. A. Lippmann and J. Schwinger, Variational principles for scattering processes, Phys. Rev. 79, 469 (1950).

[102] P. de Vries, D. V. van Coevorden, and A. Lagendijk, Point scatterers for classical waves, Rev. Mod. Phys. 70, 447 (1998).

[103] T. Savels, A. P. Mosk, and A. Lagendijk, Light scattering from three-level systems: the Tmatrix of a point-dipole with gain, Phys. Rev. A 71, 043814 (2005).

[104] H. C. van de Hulst, Multiple light scattering and radiative transfer (Academic Press, New York, 1980).

[105] M. Wubs and A. Lagendijk, Local optical density of states in finite crystals of plane scatterers, Phys. Rev. E 65, 046612 (2002).

[106] T. M. Nieuwenhuizen, A. Lagendijk, and B. A. van Tiggelen, Resonant point scatterers in multiple scattering of classical waves, Phys. Lett. A 169, 191 (1992).

[107] R. G. Newton, Scattering theory of waves and particles, 2nd ed. (Springer, New York, 1982).

[108] I. M. Vellekoop, P. Lodahl, and A. Lagendijk, Determination of the diffusion constant using phase-sensitive measurements, Phys. Rev. E 71, 056604 (2004).

[109] G. Bergmann, Weak localization in thin films; a time-of-flight experiment with conduction electrons, Phys. Rep. 107, 1 (1984).

[110] G. Bergmann, Weak localization in thin films - a time-of-flight-experiment with conduction electrons, Physica B 126, 229 (1984).

[111] D. E. Khmel'nitskii, Localization and coherent scattering of electrons, Physica B 126, 235 (1984).

[112] W. Götze, A theory for the conductivity of a fermion gas moving in a strong three-dimensional random potential, J. Phys. C 12, 1279 (1979).

[113] W. Götze, The mobility of a quantum particle in a three-dimensional random potential, Phil. 
Mag. B 43, 219 (1981).

[114] D. Vollhardt and P. Wölfle, Diagrammatic, self-consistent treatment of the Anderson localization problem in $d \leq 2$ dimensions, Phys. Rev. B 22, 4666 (1980).

[115] B. A. van Tiggelen, Multiple scattering and localization of light, Ph.D. thesis, University of Amsterdam, 1992, http://www.wavesincomplexmedia.com.

[116] B. A. van Tiggelen, A. Lagendijk, M. P. van Albada, and A. Tip, Speed of light in random media, Phys. Rev. B 45, 12233 (1992).

[117] B. A. van Tiggelen and A. Lagendijk, Rigorous treatment of the speed of diffusing classical waves, Europhys. Lett. 23, 311 (1993).

[118] S. Chandrasekar, Radiative transfer (Dover, New York, 1960).

[119] R. M. Gody and Y. L. Yung, Atmospheric radiation: theoretical basis (Oxford University Press, Oxford, 1989).

[120] P. G. de Gennes and J. Prost, The physics of liquid crystals, 2nd ed. (Oxford University Press, New York, 1993).

[121] E. Akkermans, P. E. Wolf, and R. Maynard, Coherent backscattering of light by disoredred media: analysis of the peak line shape, Phys. Rev. Lett. 56, 1471 (1986).

[122] D. J. Durian, Penetration depth for diffusing-wave spectroscopy, Appl. Opt. 34, 7100 (1995).

[123] J. H. Page, H. P. Schriemer, A. E. Bailey, and D. A. Weitz, Experimental test of the diffusion approximation for multiply scattered sound, Phys. Lett. E 52, 3106 (1995).

[124] A. Z. Genack and J. M. Drake, Relationship between optical intensity, fluctuations and pulse propagation in random media, Europhys. Lett. 11, 331 (1990).

[125] L. Tsang and A. Ishimaru, Backscattering enhancement of random discrete scatterers, J. Opt. Soc. Am. A 1, 836 (1984).

[126] L. Tsang and A. Ishimaru, Theory of backscattering enhancement of random discrete isotropic scatterers based on the summation of all ladder and cyclical terms, J. Opt. Soc. Am. A 2, 1331 (1985).

[127] L. Tsang and A. Ishimaru, Radiative wave and cyclical transfer equations for dense nontenuous media, J. Opt. Soc. Am. A 2, 2187 (1985).

[128] J. X. Zhu, D. J. Pine, and D. A. Weitz, Internal reflection of diffusive light in random media, Phys. Rev. A 44, 3948 (1991).

[129] D. S. Wiersma, M. P. van Albada, and A. Lagendijk, An accurate technique to record the angular distribution of backscattered light, Rev. Sci. Instrum. 66, 5473 (1995).

[130] S. Etemad, R. Thompson, and M. J. Andrejco, Weak localization of photons: termination of coherent random walks by absorption and confined geometry, Phys. Rev. Lett. 59, 1420 (1987).

[131] D. S. Wiersma, M. P. van Albada, B. A. van Tiggelen, and A. Lagendijk, Experimental evidence for recurrent multiple scattering events of light in disordered media, Phys. Rev. Lett. 74, 4193 (1995).

[132] F. C. MacKintosh and S. John, Coherent backscattering of light in the presence of timereversal-noninvariant and parity-nonconserving media, Phys. Rev. B 37, 1884 (1988).

[133] B. A. van Tiggelen, R. Maynard, and T. M. Nieuwenhuizen, Theory for multiple light scattering from Rayleigh scatterers in magnetic fields, Phys. Rev. E 53, 2881 (1996).

[134] R. Berkovits and M. Kaveh, Backscattering of light near the optical Anderson transition, Phys. Rev. B 36, 9322 (1987).

[135] I. Edrei and M. J. Stephen, Optical coherent backscattering and transmission in a disordered medium near the mobility edge, Phys. Rev. B 42, 110 (1990).

[136] B. A. van Tiggelen, A. Lagendijk, and D. S. Wiersma, Reflection and transmission of waves near the localization threshold, Phys. Rev. Lett. 84, 4333 (2000).

[137] Handbook of optical constants of solids, edited by E. D. Palik (Academic Press, New York, 
1952).

[138] F. Scheffold, R. Lenke, R. Tweer, and G. Maret, Localization or classical diffusion of light?, Nature (London) 398, 206 (1999), see also [179].

[139] See, for example, Journal of porous materials, (Kluwer, Dordrecht, 1997-).

[140] J. J. Kelly and D. Vanmaekelbergh, in Electrochemistry of nanomaterials, edited by G. Hodes (Wiley-VCH, Weinheim, 2001).

[141] H. Föll, S. Langa, J. Carstensen, M. Christophersen, and I. M. Titinyanu, Pores in III-V semiconductors, Adv. Mater. 15, 183 (2003).

[142] M. I. J. Beale, J. D. Benjamin, M. J. Urem, N. G. Chew, and A. G. Cullis, An experimental and theoretical study of the formation and microstructure of porous silicon, J. Cryst. Growth 73, 622 (1985).

[143] R. Herino, G. Bomchil, K. Barla, and C. Bertrand, Porosity and pore size distributions of porous silicon layers, J. Electrochem. Soc. 134, 1994 (1987).

[144] X. G. Zhang, Mechanism of pore formation on n-type silicon, J. Electrochem. Soc. 138, 3750 (1991).

[145] V. Lehmann, The physics of macroporous silicon formation, Thin Solid Films 255, 1 (1995).

[146] L. N. Aleksandrov and P. L. Novikov, Morphology of porous silicon structures formed by anodization of heavily and lightly doped silicon, Thin Solid Films 330, 102 (1998).

[147] T. Nakagawa, H. Koyama, and N. Koshida, Control of structure and optical anisotropy in porous Si by magnetic-field assisted anodization, Appl. Phys. Lett. 69, 3206 (1996).

[148] A. I. Belogorokhov, V. A. Karavanskii, A. N. Obraztsov, and V. Y. Timoshenko, Intense photoluminescence in porous gallium phosphide, JETP Lett. 60, 274 (1994).

[149] A. Anedda, A. Serpi, V. A. Karavanskii, I. N. Tiginyanu, and V. M. Ichizli, Time-resolved blue and ultraviolet photoluminescence in porous GaP, Appl. Phys. Lett. 67, 3316 (1995).

[150] S. M. Sze, Physics of semiconductor devices (Wiley-interscience, New York, 1981).

[151] R. W. Tjerkstra, J. Gómez Rivas, D. Vanmaekelbergh, and J. J. Kelly, Porous GaP multilayers formed by electrochemical etching, Electrochem. Solid State Lett. 5, G32 (2002).

[152] P. C. Searson, J. M. Macaulay, and F. M. Ross, Pore morphology and the mechanism of pore formation in n-type silicon, J. Appl. Phys. 72, 253 (1992).

[153] R. W. Tjerkstra, B. P. J. Bret, and A. Lagendijk, Eletrochemical etching of GaP in aqueous oxidizing solutions (unpublished).

[154] A. F. van Driel, D. Vanmaekelbergh, and J. J. Kelly, Electroluminescence as internal light source for measurement of the photonic strength of random porous GaP, Appl. Phys. Lett. 84, 3852 (2004).

[155] I. M. Tiginyanu, C. Schwab, J.-J. Grob, B. Prévot, H. L. Hartnagel, A. Vogt, G. Irmer, and J. Monecke, Ion implantation as a tool for controlling the morphology of porous gallium phosphide, Appl. Phys. Lett. 71, 3829 (1997).

[156] D. Vanmaekelbergh, M. A. Hamstra, and L. van Pieterson, Free carrier generation in semiconductors induced by absorption of sub-band gap light. A photoelectrochemical study with nanoporous GaP, J. Phys. Chem. B 102, 7997 (1998).

[157] T. M. Nieuwenhuizen and J. M. Luck, Skin layer of diffusive media, Phys. Rev. E 48, 569 (1993).

[158] A. Ishimaru, Wave propagation and scattering in random media (Academic Press, New York, 1978).

[159] M. U. Vera and D. J. Durian, Angular distribution of diffusely transmitted light, Phys. Rev. E 53, 3215 (1996).

[160] F. C. MacKintosh, J. X. Zhu, D. J. Pine, and D. A. Weitz, Polarization memory of multiply scattered light, Phys. Rev. B 40, 9342 (1989).

[161] D. E. Aspnes, Local-field effects and effective-medium theory: a microscopic perspective, 
Am. J. Phys. 50, 704 (1982).

[162] J. C. Maxwell Garnett, Colours in metal glasses and in metallic films, Philos. Trans. R. Soc. Lond. 203, 385 (1904).

[163] D. A. G. Bruggeman, The calculation of various physical constants of heterogeneous substances, Ann. Physik (Leipz.) 24, 636 (1935).

[164] S. Datta, C. T. Chan, K. M. Ho, and C. M. Soukoulis, Effective dielectric constant of periodic composite structures, Phys. Rev. B 48, 14936 (1993).

[165] K. Busch and C. M. Soukoulis, Transport properties of random media: a new effective medium theory, Phys. Rev. Lett. 75, 3442 (1995).

[166] K. Busch and C. M. Soukoulis, Energy-density CPA: a new effective medium theory for classical waves, Physica B 296, 56 (2001).

[167] M. Born and E. Wolf, Principles of optics (Pergamon Press, Oxford, 1986).

[168] A. F. Koenderink and W. L. Vos, Optical properties of real photonic crystals: anomalous diffuse transmission, J. Opt. Soc. Am. B 22, 1075 (2004).

[169] I. S. Nikolaev, P. Lodahl, and W. L. Wos, Quantitative analysis of directional spontaneous emission spectra from light sources in photonic crystals, Phys. Rev. E (2005), available at http://arxiv.org/abs/physics/0410056.

[170] P. Lodahl, G. van Soest, J. Gómez Rivas, R. Sprik, and A. Lagendijk, in Wave scatering in complex media: from theory to applications, edited by B. A. van Tiggelen and S. Skipetrov (Kluwer, Dordrecht, 2003).

[171] D. Stroud, Generalized effective-medium approach to the conductivity of an inhomogeneous medium, Phys. Rev. B 12, 3368 (1975).

[172] B. Abeles and J. I. Gittleman, Composite material films: optical properties and applications, Appl. Opt. 15, 2328 (1976).

[173] R. Landauer, The electrical resistance of binary metallic mixtures, J. Appl. Phys 23, 779 (1952).

[174] R. Dalichaouch, J. P. Armstrong, S. Schultz, P. M. Platzman, and S. L. McCall, Microwave localization by two-dimensional random scattering, Nature (London) 354, 53 (1991).

[175] S. He and J. D. Maynard, Detailed measurement of inelastic scattering in Anderson localization, Phys. Rev. Lett. 57, 3171 (1986).

[176] R. L. Weaver, Anderson localization of ultrasound, Wave Motion 12, 129 (1990).

[177] L. Ye, G. Cody, M. Zhou, P. Sheng, and A. N. Norris, Observation of bending wave localization and quasi mobility edge in two dimensions, Phys. Rev. Lett. 69, 3080 (1992).

[178] M. P. van Albada, B. A. van Tiggelen, A. Lagendijk, and A. Tip, Speed of propagation of classical waves in strongly scattering media, Phys. Rev. Lett. 66, 3132 (1991).

[179] D. S. Wiersma, J. Gómez Rivas, P. Bartolini, A. Lagendijk, and R. Righini, Reply to 'Localization or classical diffusion of light?', Nature (London) 398, 207 (1999).

[180] V. P. Romanov and A. N. Shalaginov, Radiation transfer equation in nematic liquid crystals, Opt. Spectrosc. 64, 774 (1988).

[181] V. P. Romanov and A. N. Shalaginov, Fluctuations and light scattering in thin smectic films, Phys. Rev. E 48, 1073 (1993).

[182] B. A. van Tiggelen, R. Maynard, and A. Heiderich, Anisotropic light diffusion in oriented nematic liquid crystals, Phys. Rev. Lett. 77, 639 (1996).

[183] H. Stark and T. C. Lubensky, Multiple light scattering in nematic liquid crystals, Phys. Rev. Lett. 77, 2229 (1996).

[184] A. Heiderich, R. Maynard, and B. A. van Tiggelen, Multiple light scattering in ordered nematic liquid crystals, J. Phys. II (France) 7, 765 (1997).

[185] B. A. van Tiggelen and H. Stark, Nematic liquid crystals as a new challenge for radiative transfer, Rev. Mod. Phys. 72, 1017 (2000). 
[186] M. H. Kao, K. A. Jester, A. G. Yodh, and P. J. Collings, Observation of light diffusion and correlation transport in nematic liquid crystals, Phys. Rev. Lett. 77, 2233 (1996).

[187] H. Stark, M. H. Kao, K. A. Jester, T. C. Lubensky, A. G. Yodh, and P. J. Collings, Light diffusion and diffusing-wave spectroscopy in nematic liquid crystals, J. Opt. Soc. Am. A 14, 156 (1997).

[188] D. S. Wiersma, A. Muzzi, M. Colocci, and R. Righini, Time-resolved anisotropic multiple light scattering in nematic liquid crystals, Phys. Rev. Lett. 83, 4321 (1999).

[189] D. S. Wiersma, A. Muzzi, M. Colocci, and R. Righini, Time-resolved experiments on light diffusion in anisotropic random media, Phys. Rev. E 62, 6681 (2000).

[190] R. Sapienza, S. Mujumdar, C. Cheung, A. G. Yodh, and D. Wiersma, Anisotropic weak localization of light, Phys. Rev. Lett. 92, 033903 (2004).

[191] M. S. Patterson, B. Chance, and B. C. Wilson, Time-resolved reflectance and transmittance for the non-invasive measurement of tissue optical properties, Appl. Opt. 28, 2331 (1989).

[192] J. Huang, N. Eradat, M. E. Raikh, Z. V. Vardeny, A. A. Zakhidov, and R. H. Baughman, Anomalous coherent backscattering of light from opal photonic crystals, Phys. Rev. Lett. 86, 4815 (2001).

[193] A. F. Koenderink, Emission and transport of light in photonic crystals, Ph.D. thesis, University of Amsterdam, 2003, http://www.wavesincomplexmedia.com.

[194] A. F. Koenderink and W. L. Vos, Optical loss due to intrinsic structural variations of photonic crystals, (2005), submitted, available at http://arxiv.org/abs/physics/0406052.

[195] P. Sebbah, B. Hu, A. Z. Genack, R. Pnini, and B. Shapiro, Spatial-field correlation: the building block of mesoscopic fluctuations, Phys. Rev. Lett. 88, 123901 (2002).

[196] R. Pnini and B. Shapiro, Fluctuations in transmission of waves through disordered slabs, Phys. Rev. B 39, 6986 (1989).

[197] V. Emiliani, F. Intonti, M. Cazayous, D. S. Wiersma, M. Colocci, F. Aliev, and A. Lagendijk, Near-field short-range correlation in optical waves transmitted through random media, Phys. Rev. Lett. 90, 250801 (2003).

[198] C. J. Oton, Z. Gaburro, M. Ghulinyan, L. Pancheri, P. Bettotti, L. Dal Negro, and L. Pavesi, Scattering rings in optically anisotropic porous silicon, Appl. Phys. Lett. 81, 4919 (2002).

[199] M. P. van Albada, M. B. van der Mark, and A. Lagendijk, Observation of weak localization of light in a finite slab: anisotropy effects and light path classification, Phys. Rev. Lett. 58, 361 (1987).

[200] D. S. Wiersma, S. Gottardo, R. Sapienza, S. Mujumdar, S. Cavalieri, M. Colocci, R. Righini, L. Dal Negro, C. Oton, M. Ghulinyan, Z. Gaburro, L. Pavesi, F. Aliev, P. M. Johnson, A. Lagendijk, and W. L. Vos, in Wave scatering in complex media: from theory to applications, edited by B. A. van Tiggelen and S. Skipetrov (Kluwer, Dordrecht, 2003).

[201] M. J. Stephen and G. Cwilich, Rayleigh scattering and weak localization: effects of polarization, Phys. Rev. B 34, 7564 (1986).

[202] L. V. Kuzmin, V. P. Romanov, and L. A. Zubkov, Coherent backscattering from anisotropic scatterers, Phys. Rev. E 54, 6798 (1996).

[203] H. K. M. Vithana, L. Asfaw, and D. L. Johnson, Coherent backscattering of light in a nematic liquid crystal, Phys. Rev. Lett. 70, 3561 (1993).

[204] M. P. van Albada, M. B. van der Mark, and A. Lagendijk, Polarization effects in weak localization of light, J. Phys. D 21, 28 (1988).

[205] G. Labeyrie, F. de Tomasi, J.-C. Bernard, C. A. Müller, C. Miniatura, and R. Kaiser, Coherent backscattering of light by cold atoms, Phys. Rev. Lett. 83, 5266 (1999).

[206] F. Brandi, I. Velchev, W. Hogervorst, and W. Ubachs, Vacuum-ultraviolet spectroscopy of Xe: hyperfine splittings, isotope shifts, and isotope-dependent ionization energies, Phys. Rev. A 64, 032505 (2001). 
[207] R. Zinkstok, E. J. van Duijn, S. Witte, and W. Hogervorst, Hyperfine structure and isotope shift of transitions in $\mathrm{Yb}$ I using $U V$ and deep-UV cw laser light and the angular distribution of fluorescence radiation, J. Phys. B 35, 2693 (2002).

[208] G. D. Boyd and D. A. Kleinman, Parametric interaction of focused gaussian light beams, J. Appl. Phys. 39, 3597 (1968).

[209] H. Park, J. Lee, J.-H. Lee, and J.-S. Chang, Selective photoionization of the ytterbium atom by coherent two-photon excitation, Phys. Rev. A 53, 1751 (1996).

[210] S. K. Borisov, M. A. Kuz'mima, and V. A. Mishin, A study of isotopically selective photoionization of ytterbium atoms for laser isotope separation, J. Russ. Laser Res. 17, 332 (1996).

[211] A. Siegman, Lasers (University Science Books, New York, 1986).

[212] D. F. Phillips, A. Fleischhauer, A. Mair, R. L. Walsworth, and M. D. Lukin, Storage of light in atomic vapor, Phys. Rev. Lett. 86, 783 (2001).

[213] C. Liu, Z. Dutton, C. H. Behroozi, and L. V. Hau, Observation of coherent optical information storage in an atomic medium using halted light pulses, Nature (London) 409, 490 (2001).

[214] M. F. Yanik and S. Fan, Stopping light all optically, Phys. Rev. Lett. 92, 083901 (2004).

[215] M. F. Yanik and S. Fan, Stopping and storing light coherently, Phys. Rev. A 71, 013803 (2004).

[216] G. R. Fowles, Introduction to modern optics (Dover, New York, 1968).

[217] T. W. Hänsch and B. Couillaud, Laser frequency stabilization by polarization spectroscopy of a reflecting reference cavity, Opt. Commun. 35, 441 (1980).

[218] H. W. Kogelnik, E. P. Ippen, A. Dienes, and C. S. Shank, Astigmatically compensated cavities for CW dye lasers, IEEE J. Quantum Electron. QE-8, 373 (1972). 


\section{Summary}

This thesis presents an experimental study on multiple light scattering, with the necessary introductions: theoretical background and sample preparation. The emphasis is put on the effects of the multiple scattering of waves, i.e., where interference effects exist and are significant, in the search for Anderson localization.

The principles of multiple-scattering theory are presented. Without interference, when the scattering strength of the medium increases, the propagation of light turns from ballistic via single scattering to the diffusion regime. In a stationary measurement, such as performed with a continuous-wave laser or a light bulb, diffusion is characterized by the mean free path $\ell$, the average distance for light to scatter in all directions. In a dynamic measurement, such as performed with a pulsed-laser source and a time-resolved detection, the characteristic quantity is the diffusion constant (which is a length times a speed).

There are various effects of interference in multiple scattering, but we focus on the enhanced backscattering (EBS). The EBS arises from constructive interference of reciprocal (or time-reversed) paths in ensemble-averaged disordered media. The light reflected from a diffusive sample has a typical broad Lambertian shape on top of which a narrow cone at exact backscattering is present. The width of this EBS cone is characterized by the mean free path and the wavelength of the light $\lambda$. The top of the EBS cone gives information about the coherence lengths inside the material: the finite size, the absorption length, and the localization length.

The regime of multiple light scattering is studied in a porous semiconductor, gallium phosphide $(\mathrm{GaP})$, known at present as the strongest scattering material for visible light (around $633 \mathrm{~nm}$ ). A wafer of $\mathrm{GaP}$, doped with sulfur, is electrochemically etched to produce a porous structure of homogeneous thickness. The understanding of the chemistry of this etching process is presented. The properties of the porous structure depend mainly on the doping concentration of the wafer and the electrical voltage applied during etching. The pores can be made with a diameter in the range 50 to $200 \mathrm{~nm}$, thus smaller than the wavelength of light. The porous samples are then further photochemically etched, in order to remove a thin layer of bare GaP remaining on top of the porous structure after electrochemical etching. The removal of this top-layer simplifies the interpretation of optical measurements on the porous samples. The porous samples can also be further chemically etched, in order to homogeneously increase the average diameter of the pores, toward the wavelength of light.

The three types of samples, after electrochemical, photochemical and chemical etching, have been characterized by several optical techniques, among which the total transmission, the EBS, and the angular-resolved transmission measurements. A very important parameter 
of diffusive samples is the effective refractive index, which modifies both the wave vector of light inside the material and the internal reflection at the interface. A theory which carefully treats the boundary conditions of a diffusive medium is presented. This theory predicts that the angular-resolved transmission is characteristic of the refractive index contrast at the interface between the diffusive medium and the outside medium. The refractive index of strongly scattering porous samples, after photochemical etching, is determined by fitting the theory to the angular-resolved transmission data. The refractive index as a function of porosity in the domain of strong scattering is experimentally studied. The effective medium theories, rigorously derived only in the weak-scattering limit, fail to describe our measurements of the refractive index.

In the search for Anderson localization of light, very strongly scattering samples are produced by electrochemical etching. The optical absorption length is shown to exceed the thickest samples produced, namely $250 \mu \mathrm{m}$. The strongest scattering samples are made from GaP wafers of doping concentration $N=2-5 \times 10^{17} \mathrm{~cm}^{-3}$, electrochemically etched at the highest possible voltage. The average diameter of the pores in a sample is increased by chemical etching. The scattering strength of certain samples increases after chemical etching. A careful study of the total transmission and the width and rounding of the EBS cone shows, before and after chemical-etching, a very good agreement of the measurements with the diffusion regime. Within the scope of this thesis, no effect which can be attributed to Anderson localization has been recorded, even for very strongly scattering samples where $\ell / 2 \pi \lambda \simeq 3.5$.

The electrochemical etching produces a porous structure with oriented pores: pores grow in the direction normal to the surface of the GaP wafer. The geometrical anisotropy induces anisotropic diffusion. An anisotropic hopping model and subsequent diffusion theory with an anisotropic diffusion constant tensor and an isotropic mean free path is presented. The anisotropy in both stationary and dynamic measurements are predicted to depend on the components of the diffusion constant tensor. Measurements on anisotropic samples are indeed anisotropic for stationary and dynamic diffusion, and EBS. The anisotropy in the diffusion constant tensor, independently determined from these three optical measurements, is consistent. Porous GaP displays both strong scattering and strong anisotropy (the ratio of the diffusion constant components is about 4).

The last part of this thesis deals with a subject differing from multiple light scattering. The subject of the full capture of a light pulse inside a short cavity is considered, and can be seen as a macroscopic equivalent of the switching of an (Anderson) localized state. In a short cavity, the bandwidth of the incident pulse is decreased, according to the finesse of the cavity, at the expense of the incoupling efficiency. By dynamically adapting the reflectivity of the input coupler to the shape of the incident pulse, the light reflected from the cavity can be made to vanish by destructive interference. When no light is reflected off the cavity, all the incident power is coupled inside the cavity, within a single mode of narrow bandwidth. A theoretical description of the pulse capture inside a short high-finesse cavity is presented. A practical implementation of the mirror with variable reflectivity is made by using a Pockels cell and polarization optics, along with a fast high-voltage switch. The experimental results in the frequency-domain show both a higher power and a narrower bandwidth of the light pulse captured in the cavity, compared to the same setup with any constant reflectivity of the input coupler. 


\section{Samenvatting}

\section{Meervoudige verstrooiing van licht in poreus gallium fosfide}

In dit proefschrift wordt een experimenteel onderzoek van meervoudige verstrooiing van licht gepresenteerd, met de noodzakelijke introducties: de theoretische achtergrond en de monsterpreparatie. De nadruk is op de effecten van meervoudige verstrooiing van golven, waar interferentie bestaat en belangrijk is, in de zoektocht naar Anderson lokalisatie.

De principes van de theorie van meervoudige verstrooiing worden uitgelegd. Zonder interferentie, als de kracht van de verstrooiing van een media toeneemt, verandert de voortplanting van licht van ballistisch naar enkele verstrooiing, tot naar het diffusie-regime. In een stationaire meting, zoals gedaan met een continue laser of een gloeilamp, wordt de diffusie gekarakteriseerd door de gemiddelde vrije weglengte $\ell$, oftewel de gemiddelde afstand dat licht nodig heeft om in alle richtingen te verstrooien. In een dynamische meting, zoals gedaan met een gepulste laserbron en een tijdopgeloste detectie, is de karakteristieke grootheid de diffusieconstante (die een lengte keer een snelheid is).

De effecten van interferentie in meervoudige verstrooiing zijn divers, maar we benadrukken hier de terugstrooikegel (TSK). De TSK komt door de constructieve interferentie van wederkerige (of tijdomgekeerd) paden in ensemble-gemiddelde wanordelijke media. Het gereflecteerde licht van een diffuus monster heeft een typische Lambertiaanse vorm waarop een nauwe kegel staat in de precieze terugstrooirichting. De breedte van die TSK is gekarakteriseerd door de gemiddelde vrije weglengte en de golflengte van het licht $\lambda$. De top van de TSK geeft informatie over de coherentielengten in het materiaal: de eindige dikte, de absorptielengte, en de lokalisatielengte.

Het regime van meervoudige verstrooiing van licht is in een poreuze halfgeleider, gallium fosfide $(\mathrm{GaP})$, bestudeerd, die tegenwoordig bekend staat als het sterkst verstrooiende materiaal voor zichtbaar licht (rond $633 \mathrm{~nm}$ ). Een wafer van $\mathrm{GaP}$, gedoteerd met zwavel, is electrochemisch geëtst om een poreuze structuur met gelijkmatig dikte te maken. De chemie van die etsproces wordt besproken. De eigenschappen van de poreuze structuur hangen allereerst af van de dotering van de GaP wafer en de spanning die tijdens etsen is aangelegd. De poriën zijn gemaakt met een maat tussen 50 en $200 \mathrm{~nm}$, en dus kleiner dan de golflengte van het licht. De poreuze monsters zijn daarna fotochemisch geëtst om een dunne toplaag te verwijderen, die op de poreuze laag overblijft na het electrochemische 
etsen. De verwijdering van die toplaag vereenvoudigt de interpretatie van de optische metingen aan de poreuze monsters. De poreuze monsters kunnen ook extra chemisch geëtst worden, zodat de gemiddelde doorsnede van de poriën gelijkmatig toeneemt, tot dicht bij de golflengte van het licht.

Drie typen van monsters, na electrochemische, fotochemische, en chemische etsen, zijn gekarakteriseerd door enkele optische technieken, met name de totale transmissie, de TSK, en de hoekopgeloste transmissiemetingen. Een heel belangrijke parameter van de diffuse monsters is de effectieve brekingindex, waarvan zowel de golfvector van licht in het materiaal als de interne reflectie aan het oppervlak afhangen. Een theorie wordt gepresenteerd die de randvoorwaarden van een diffuus medium zorgvuldig behandelt. Deze theorie voorspelt dat de hoekopgeloste transmissie wordt gekarakteriseerd door het brekingsindexcontrast van het oppervlak tussen het diffuse en het buitenmedium. De brekingsindex is experimenteel onderzocht als functie van porositeit in het domein van sterke verstrooiing . De effectieve-mediumtheorieën, zorgvuldig afgeleid in de limiet van zwakke verstrooiing, beschrijven onze metingen van brekingsindex niet goed.

In de zoektocht naar Anderson lokalisatie van licht, zijn heel sterk verstrooiende monsters gemaakt door middel van electrochemische etsen. De optische absorptielengte is langer dan de dikste monsters, namelijk $250 \mu \mathrm{m}$. De sterkst verstrooiende materialen zijn gemaakt van GaP wafers met dotering $N=2-5 \times 10^{17} \mathrm{~cm}^{-3}$, en electrochemisch geëtst op de hoogste mogelijke spanning. De gemiddelde doorsnede van de poriën in een monster neemt toe met chemische etsen. De verstrooiingkracht van sommige monsters neemt toe met het toepassen van chemische etsen. Een zorgvuldige bestudering van de totale transmissie en de breedte en afronding van de TSK toont, voor en na chemische etsen, een heel goede overeenstemming van de metingen met het diffusie-regime. Binnen de ruimte van dit proefschrift is geen effect gemeten, dat door Anderson lokalisatie veroorzaakt kan zijn, zelfs niet voor heel sterk verstrooiende monsters, waar $2 \pi \ell / \lambda \simeq 3.5$.

Het electrochemische etsen maakt een poreuze structuur met gerichte poriën: poriën groeien in de richting loodrecht aan het oppervlak van de $\mathrm{GaP}$ wafer. De geometrische anisotropie veroorzaakt anisotrope diffusie. Een anisotroop sprongmodel en de daaruitvolgende diffusie-theorie met een anisotrope diffusieconstante tensor en isotrope gemiddelde vrije weglengte wordt afgeleid. De anisotropie in beide stationaire en dynamische metingen zijn voorspeld afhankelijk te zijn van de componenten van de diffusie-tensor. Metingen aan anisotrope monsters zijn inderdaad anisotroop voor stationaire en dynamische diffusie, en voor TSK. De anisotropie in de diffusie-tensor, als gemeten van deze drie onafhankelijke optische metingen, is consistent. Poreus GaP toont zowel sterke verstrooiing als sterke anisotropie (de verhouding van de diagonale elementen van de diffusie-tensor is rond 4).

De laatste deel van dit proefschrift pakt een onderwerp aan dat verschilt van meervoudige verstrooiing van licht. Het onderwerp van het volledige opslaan van een lichtpuls binnen een korte trilholte wordt behandelt. Dit verschijnsel kan gezien worden als een macroscopisch equivalent van de schakeling van een (Anderson) gelokaliseerde toestand. In een korte trilholte neemt de bandbreedte van de inkomende puls af volgens de finesse van de trilholte, ten koste van de inkoppeling efficiëntie. Door dynamische schakeling van de reflectiviteit van de invoerkoppelaar volgens de vorm van de inkomende puls, kan de reflectie van de trilholte worden voorkomen door destructieve interferentie. Als geen licht door de trilholte wordt gereflecteerd, wordt het gehele inkomende vermogen opgenomen in de tril- 
holte, binnen een enkele toestand met nauwe bandbreedte. Een theoretische beschouwing van het vangen van de puls in een kort trilholte met een hoge finesse wordt gegeven. Een praktische implementatie van de inkoppelspiegel met variabele reflectiviteit is doorgevoerd met een Pockels cel en polarisatie-optica, samen met een snelle schakeling van de hoogspanning. De proefresultaten, gemeten als functie van frequentie, tonen tegelijk een hoger vermogen en nauwere bandbreedte van het opgenomen licht, in vergelijking met dezelfde opstelling maar met een willekeurige constante reflectiviteit van de invoerkoppelaar. 



\section{Acknowledgements}

This is the last, and most important, part of my thesis. All the physics I wrote in this book hardly reflects what I have experienced and learned during these last four years. It is also the time to thank all the people without whom my thesis would be but a blank page.

Allereerst wil ik mijn promotor, Ad Lagendijk, van harte bedanken. Jouw inzicht in wetenschap, jouw kritisch ondersteuning, maar ook jouw manier om een baas te zijn, en om de vuist op de tafel te slaan, waren allen inspirerend. Het was niet altijd makkelijk om met jou te werken, maar altijd ontzettend leerzaam. Ik ben met jou en de groep naar Enschede verhuisd, en dat zou ik nog een keer doen als het nodig zou zijn. Willem Vos (le renard?), medebestuurder van ons groep COPS maar zeker ook vriend, dank ik voor het, onverwacht, uitnodiging om te komen solliciteren in Amsterdam. Zonder jou was ik gewoon niet in de groep gekomen om te promoveren, en had ik ook niet zo veel geleerd.

Mijn primair onderzoek heb ik gedaan met mijn mede-etsers: Jaime, Willem en Patrick. Jaime, je hebt mij alles geleerd over poreus GaP, over de optische en etsen opstellingen, jij had verdiend om lokalisatie van licht te meten. Willem, bedankt voor de monsters en jouw constante stimulering: 'Is je proefschrift al af?'. Patrick, your idea of making anisotropic samples proved very fruitful, as were other discussions about juggling and unicycling.

Met Tom heb ik over een ander ontwerp en opstelling gewerkt en geknutseld, die tot mijn laatste hoofdstuk hebben geleid. Bedankt voor dit heel aardige experiment, en voor mijn ontdekking van sportief klimmen. Allard wil ik bedanken voor de lange en diepe discussies die uit een kleine vraag zijn gekomen, en ook voor de ondersteuning wanneer mijn kegelopstelling verkouden was van luchtvochtigheidfluctuaties.

Praktische hulp heb ik van veel verschillende mensen gekregen, in Enschede en in Amsterdam. Cock, vrolijke meester van het COPS-lab, de vijand van dubbelzijdig plakband. Raymond, onze manager en anti-nerd. Karen voor de administratie die wij altijd liever niet zelf doen. Bas de computerbaas. Onze buurgroep de werkplaats, en in het bijzonder Klaas voor de leuke uren van knutselen, de altijd perfecte nabewerking, en de gezellige discussies. Wim, de meester van het Amsterdamse lab, en een van de beste reden voor de groep om in het WZI te blijven. Mariet die alle administratie tussen mijn verhuizing naar Amsterdam en de verhuizing naar Enschede heeft geregeld.

Met de electro-chemie groep uit Utrecht heb ik goede samenwerking gehad. Bedankt Floris, John et Daniël.

Het beste deel van in Amsterdam, en dan in Enschede, te zijn was de groep zelf. Bedankt voor de gezelligheid, de taarten voor de (talrijke) speciale gelegenheden, de inspir- 
erend groepsdiscussies, de film- en sportavonden ${ }^{7}$ en sorry voor de druk die mij te weinig tijd heb gelaten om koffiepauzes met jullie te hebben. Van de rest van de groep, in Amsterdam en in Enschede, met name Arie, Bart, Bernard, Denis, Emmanuèle, Femius, Gerard, Gijs, Ivan, Ivo, Juan, Karen, Karin, Léon, Lydia, Martijn, Peter, Peter, Philip, Rob, Rudolf, Tijmen, Timo, Tom, Valentin, Vitaly, Wouter, Yuri, ga ik ook heel goede herinneringen houden.

Ik wil ook graag alle Nederlanders bedanken die met mij geen Frans of Engels hebben gesproken, en die mijn Nederlands hebben gecorrigeerd.

Gelukkig kon ik ook af en toe mijn hoofd uit het onderzoek zetten. Ik wil vooral alle jongleurs van de vereniging CatchUp bedanken, voor de gezelligheid van onze ontmoetingen/traninginen, de stimulering om ballen en kegels beter omhoog te gooien, en het plezier om samen te jongleren/eenwieleren/acrobatieken. Met de klimmers heb ik ontdekt dat spieren die je niet kent, ook pijnlijk kunnen zijn, en hoe een biertje en een leuk praatje daarna een perfect aanvulling is op sporten.

Où serais-je aujourd'hui sans la présence fidèle et encourageante de mes parents? Un énorme merci pour votre éducation, vos conseils, votre soutien, l'opportunité que vous m'avez donnée d'étudier et de poursuivre mes passions. Et merci à maman pour les consultations-minute par webcam! Une pensée chaleureuse à mon nano- mais néanmoins grand frère, Tristan, qui a bataillé en même temps que moi sur sa thèse. Quant à ma sœur, Lætitia, merci pour la cuisine de chef et l'envie de faire des expériences culinaires.

Sandrine, la plus belle chose qui me soit arrivée pendant ma thèse. De conduire chacun notre thèse en même temps, avec ses lenteurs, ses pressions, ses joies, ses frustrations, ses doutes, sa libération, nous a rapprochés plus encore que si nous avions vécu ensemble. C'est notre victoire à nous! Obrigado por tudo.

Boris Bret, 2005.

\footnotetext{
${ }^{7}$ Ik kan mijn/ons titel van biljart en volleybal dit jaar niet verdedigen, laat het beste winnen!
} 